\title{
Stamping Out Disease: Health Education and Promotion by Miniature Messengers
}

By

\section{Kenneth Porter Dawson}

\author{
A thesis \\ Submitted to the Victoria University of Wellington \\ In fulfilment of the requirement for the degree of \\ Master of Health Research
}

Victoria University of Wellington

2021 


\section{Frontispiece}

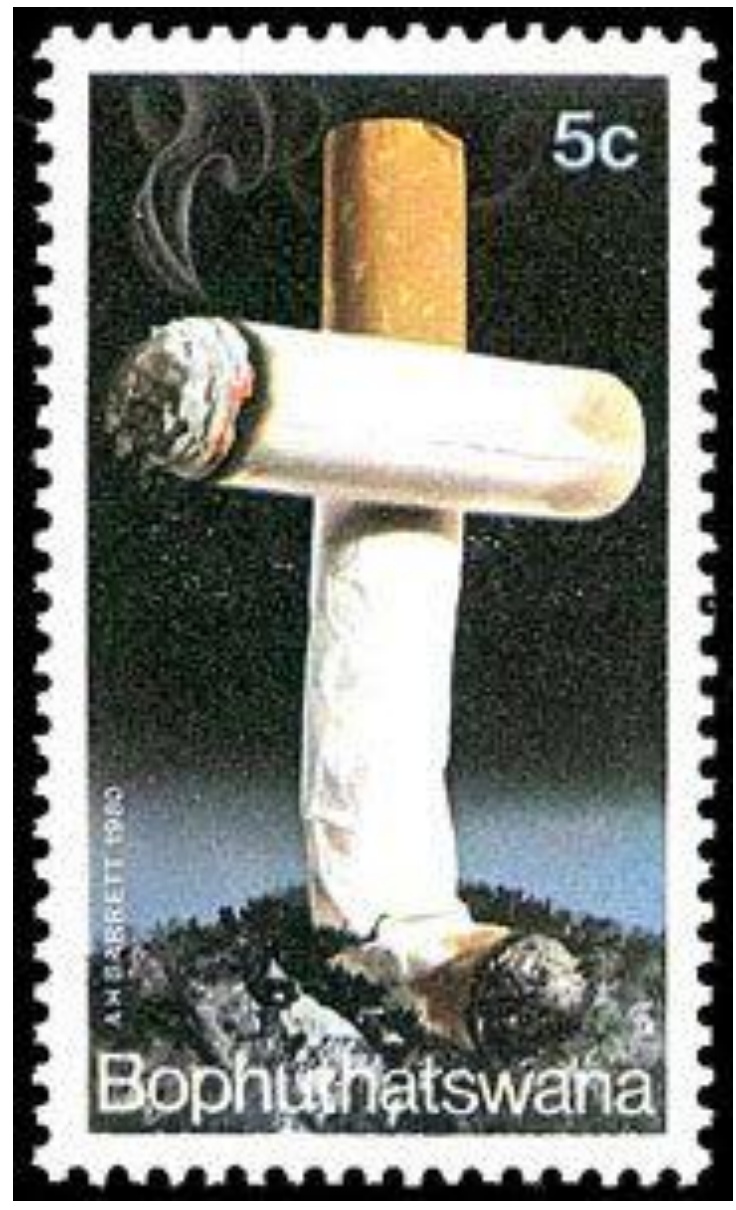

Anti-smoking campaign, an example of the visual power of the postage stamp Bophuthatswana, 1980 "Stop smoking”. 


\section{Abstract}

This study explores the role of the ubiquitous postage stamp as a messenger of health promotion and education. It examines the way that postal material has been modified to act as a medium for health education, with images and texts appropriate to the specific health problems. The methodology used included a search for all postage stamps listed in established catalogues that fitted the described criterion. Selection for further study was drawn from those stamps that covered the topics of infection, immunisation, tobacco cessation, growth and nutrition.

It was clearly recognised that by simply presenting information on a stamp, would not necessarily bring about the desired behavioural change. Thus, the currently accepted health promotion models were examined to find an appropriate explanation for/ and a rationale to explain the use of postage stamp in health promotion and education. The strategy called "media advocacy" was the nearest best fit for the bulk of postage stamps carrying health information. This finding could explain why stamps have been perceived, over many years, as being a useful and successful element in health promotion and education by their frequent and continued use over a period of some fifty years.

Postage stamps are frequently used worldwide and have a clear role in carrying health information and thus play a small, but important and unique, role in the field of health promotion. 


\section{Acknowledgements}

I wish to acknowledge the support and encouragement my wife, Mairi, has given me in pursuing further studies in the health field. I shall be forever grateful to her for her constant support and tolerance.

I wish to thank Dr. Eva Neely for her support and input during the formation of this dissertation. 


\section{Table of Contents}

$\begin{array}{lr} & \text { Page } \\ \text { Frontispiece } & \text { ii } \\ \text { Abstract } & \text { iii } \\ \text { Acknowledgements } & \text { iv } \\ \text { Table of Contents } & \text { v } \\ \text { List of Figures } & \text { vi - vii } \\ \text { List of Tables } & \text { viii } \\ \text { Introduction } & 1 . \\ \text { Methodology } & 6 . \\ \text { Chapter 1. The Miniature Messenger } & 11 . \\ \text { Chapter 2. A New Role for the Postage Stamp: a Health Messenger, Delivering a } & \\ \quad \text { Textual Message in the Promotion of Health. } & 20 . \\ \text { 2.1. The Health Messenger } & 20 . \\ \text { 2.2. Delivering a Textual Message } & 25 . \\ \text { 2.3. The Promotion of Health by Postage Stamps: A Background Review } & 31 .\end{array}$

Chapter 3. Health Education Through Awareness: What Constitutes a Health Educational or Promotional Postage Stamp.

Chapter 4. Heath Campaigns: an Important Role for the Postage Stamp as a Health Messenger.

Chapter 5. Health Campaigns: the Role of the Postage Stamp as an Anti-smoking Health Messenger.

Chapter 6. Postage Stamps Promoting Immunisation and Disease Control

Chapter 7. Miniature Messages Promoting Nutrition, Growth and Development in Childhood.

7.1. Breast Feeding

7.2. Child Nutrition and Growth

Chapter 8. Health Promotion and Postage Stamps: Changing Concepts Over Time.

90.

Discussion

Conclusion

Bibliography 


\section{List of Figures}

Page

Frontispiece

ii

1. Stamp cancellations from Singapore and United Kingdom.

2. Examples of health charity stamp, New Zealand and Red Cross celebration stamp.

3. Stamps depicting culture scenic beauty and history.

4. Nicaraguan stamp of 1937, the Honduran stamp of 1943.

14.

5. Examples of First World War, occupation overprints.

15.

6. German propaganda stamps.

7. Basic requirements for a postage stamp.

18.

8. Anti-drugs campaign issue illustrating the danger of drugs.

19.

9. The first health stamps of New Zealand.

21.

10 . The earliest charity stamps.

22.

11. Tuberculosis related stamps with additional overprints.

23.

12. Buy anti-tuberculosis stamps.

24.

13. Encouraging BCG immunisation.

24.

14. The Cuban artist Silvia Fernández Arrojo.

25.

15. Innovations in medicine series.

25.

16. Meghdoot postal card, India.

26.

17. United States cancer stamps.

27.

18. Prevention of breast cancer.

29.

19. Semi-postal stamp label attached, to promote BCG immunisation. 30.

20. Examples of text messages. 30.

21. Semi-postal stamp for breast cancer charities. 30.

22. Health related stamps that lack a direct health message. 32.

23. Infant growth monitoring.

33.

24. Postage stamps with a medical subject.

33.

25. Stamp scores.

34.

26. Eradication of smallpox.

43.

27. Indication that these stamps carry a surcharge for the Tuberculosis Fund.

45.

28. Eye-related themes of some educational value.

45.

29. New malaria stamps from Taiwan.

48.

30. Stages of the mosquitoes' development. 50.

31. Malarial breeding. 51.

32. Anopheles mosquito portrayed by Timor and Dubai. 52.

33. Elimination of the mosquito by insecticide spraying. 54.

34. Mosquito nets.

35. Malaria: Diagnosis and treatment. 56.

36. Mosquito superimposed on postage stamp. 57.

37. Slogan stamp cancellation. 58.

38. Mosquito elimination. 59.

39. Teatment and management of malaria. 61 .

40. Mankind's burden. 63.

41. Anti-smoking stamps. 65. 
42. The first anti-smoking postage stamp, Czechoslovakia, 1975.

65.

43. Number of anti-smoking stamps issued per year, 1977-2019.

66.

44. Dramatic use of images, Czechoslovakia, 1981, Indonesia, 1991, Italy, 1982.

67.

45. Anti-smoking and pro-smoking images.

69.

46. Original photographs and postage stamps.

70.

47. "Do not smoke for the health of you and your beloved ones".

71.

48. Contrasting anti-smoking images.

72.

49. Range of immunisations available.

74.

50. A world without polio.

75.

51. The crippling effects of polio.

76.

52. Polio vaccine development.

77.

53. Sabin and polio vaccine development. 78.

54. Oral polio vaccine.

79.

55. Celebrating both Sabin and Salk.

56. Woman breast feeding.

57. Breast is best. $\quad 82$.

58. Breast feeding in various geographic locations. 85 .

59. Fight against malnutrition, Ghana, 1973.

60. Growth monitoring. 87.

61. Better nutrition. 88.

62. Diseases on postage stamps. 94.

63. Leprosy stamps. 95.

64. Corneal transplantation, eye anatomy, blindness and malnutrition. 96.

65. River blindness and eye examination. 97.

66. Radiology advances and community health promotion. 98.

67. Current health topics. 98.

68. Stamps related to current health problems. 102.

69. Stamps against blindness and AIDS. 104.

70. Humour and smoking 108. 


\section{List of Tables}

Page

1. Question, Score and Assessment Sheet. 37.

2. Results of Score Sheet Assessment for the 19 Postage Stamps. 40. 


\section{Introduction}

To link postage stamps with health promotion would appear, at first sight, to be an unusual association. The purpose of this dissertation is to demonstrate that there is, in fact, an important historical, relevant and on-going connection between the two. Postage stamps have impact and the reference literature continues to expand in relation to this impact in politics, especially geo-politics, and their use as propaganda agents during war and in particular World War 2. ${ }^{1}$ While the primary function of the postage stamp is the prepayment of mail, a secondary function soon developed as that of being a miniature messenger. The stamp's ubiquitous nature, the vast improvements in design and printing techniques that have occurred in the last number of years, had created the potential of making it an attractive way of delivering health messages.

Postage stamps were introduced into the United Kingdom in 1840, and were rapidly accepted worldwide, as convenient way of paying mail charges and have continued to be used in this role to the present day. In New Zealand, for example, the peak volume of letter use was in 2008 , when 1,000 million letters were posted. ${ }^{2}$ Since then there has been a steady decline in the volume of letters posted in the subsequent years, and this is generally regarded as due to the development and increase in the use of electronic mail. It could be argued that the decline in letter postage would result in a decline in postage stamp production and the relevance of the postage stamp. In reality, the overall number of postage stamps produced has, however, increased as have the individual numbers in each issued set. ${ }^{3}$ Along with this change has been an expansion in the number of postal authorities who issue postage stamps. This paradox will be explained in a later chapter and postage stamps have continued to be bearers of health information to the present day.

Can a study of the relationship between stamps and health promotion be justified on academic grounds? The literature would strongly support this being so. The evidence that postage stamps convey messages that have impact has been presented in a number of different fields and disciplines. Raento and Brunn showed that stamps have helped promote

\footnotetext{
1 Jack Child, Miniature messengers, Durham: Durham University Press, 2008: 1-12.

${ }^{2}$ A. H. McLintock (ed.) "Mail services", An Encyclopaedia of New Zealand, Wellington: Ministry of Culture and Heritage, 1966.

${ }^{3}$ Richard Lehmann, "The future of philately", Forbes Magazine, 28 August, 2016. Eugene L. Meyer, "Stamped out", New York Times, 29 September, 2017.
} 
changes in Finland's society and its politics and have nation-building power. ${ }^{4}$ Michael Billig has stated that routine symbols such as stamps reinforce people's feeling of national identity, and Stanley Brunn has added that stamps are among the most important early symbolic decisions to be made at state level. ${ }^{5}$ It has been further shown that stamps can provide visual evidence of historical events and that political agendas can be changed through their texts and images. ${ }^{6}$ Additionally, Stanley Brunn has introduced the concept of 'visual geopolitics' and that postage stamp issues can be used to present views about the global environment, such as shrinking polar icecaps, threatened habitats and temperature change.$^{7}$

From the above it would appear that it is now well established that the study of postage stamps has academic standing and hence there is merit in a study of their potential connection to health promotion. It is, however, important to define what is meant by health promotion in the context of this research before proceeding further.

The term 'health promotion' was first coined by Henry Sigerst in 1945. Since then, health promotion has become established as an important aspect of the health and social services of nation states. ${ }^{8}$ Attempts to define what constitutes health promotion have been made, and later expanded, following two World Health Organisation congresses. The first of these was held in Ottawa in 1986 and a second in Bangkok in 2006. ${ }^{9}$ A part of the agreed definition of health promotion states: The process of enabling people to increase control over their health and its determinates and thereby improve their health. The Charter

\footnotetext{
${ }^{4}$ Pauliina Raento, Stanley D. Brunn, "Visualizing Finland: postage stamps as political messengers", Geografiska Annaler, Series B, Human Geography, 2005; 87: 159.

${ }^{5}$ Michael Billig, Banal nationalism, New York: Sage Publishing, 1995.

Stanley Brunn, "Stamps as messengers of political transition", Geographical Review, 2011; 101: 19-36.

${ }^{6}$ Phil Deans, Hugo Dobson, "Introduction: East Asian postage stamps as socio-political artefacts", East Asia, 2005; 22: 3-7.

7 Stanley D. Brunn, "A geopolitical and geovisualization challenge: increasing awareness of global environmental changes through postage stamps". Natural Resources. 2017; 8: 130-158.

${ }^{8}$ Editorial, "What is health promotion?" Journal of Public Health Policy, 1986; 7: 147-51. Ralph DiClemente, James Raczynski, "The importance of health promotion and disease prevention", in Raczynski and DiClemente (eds.) The Handbook of Health Promotion and Disease Prevention, Boston: Springer, 1999: 3-9.

Sanjiv Kumar, GS Preetha, "Health promotion: an effective tool for global health", Indian Journal of Community Medicine, 2012; 37: 5-12.

9 World Health Organisation, "Ottawa Charter for health promotion", Canada Journal of Public Health, 1986; 77: 425-430. https://www.who.int/healthpromotion/conferences/en/ accessed 8/10/2020. WHO Bangkok Charter for health promotion, https://www.who.int/healthpromotion/conferences/6gchp/bangkok charter/en/ accessed 6/5/2020.
} 
stressed three elements of health promotion (1) advocacy for health (2) enabling people to achieve their full health potential (3) mediation between different interests in society for the pursuit of health. ${ }^{10}$ Subsequently, the concept of health literacy developed as one aspect of health promotion. Health literacy has been defined as the capacity to obtain, process and understand basic health information and services in order to make informed and appropriate health decisions. Three aspects of this concept are (1) provision of information (2) understanding the information and deciding on its accuracy and sufficiency (3) to act on the information. ${ }^{11}$ These definitions are important as Peter Howat et al. have stressed there is a need for consensus views on what defines aspects of health promotion to prevent confusion between the various health professions involved in health promotion. ${ }^{12}$ There is also the important further factor, when designing health promotion initiatives, that interventions to address socio-economic determinants of health can have the greatest impact. ${ }^{13}$

This dissertation is concerned with the examination of postage stamps as one unheralded way of the provision of information and its ability to convey an understanding of the information provided. Acquiring accurate information is an important component of enabling healthy choices. To date, health information has been imparted in a host of ways such as from social media, on-line articles, published books, films, radio and television. With regard to those health workers who are responsible for providing information for these media outlets, there is a need for a common minimum set of competencies as there are differing professionals involved. ${ }^{14}$

In global terms there is a great range of cultures, and differing levels of educational attainment that have to be considered when imparting health information. In addition, there are the confounding factors of the geography of disease, such as malaria in the

\footnotetext{
10 Fran Baum, The new public health, Oxford: Oxford University Press, 2016: 39.

${ }^{11}$ Ministry of Health, Health literacy review: a guide, Wellington; Ministry of Health, 2015.

12 Peter Howat, D. Cross, B. Maycock et al., "Towards a more unified definition of health promotion", Health Promotion Journal of Australia, 2003; 14: 82-84.

${ }^{13}$ Thomas R. Friedman, "A framework for public health action; the health impact pyramid", American Journal of Public Health, 2010; 100: 590-595.

${ }^{14}$ Ann Wylie, Kathleen Leedham-Green, "Health promotion in medical education: lessons from a major undergraduate curriculum implementation", Education for Primary Care, 2017; 28: 325-333. David M. Morris, Gavin R. Jenkins, "Preparing physical and occupational therapists to be health promotion practitioners: a call for action", International Journal of Environmental Research and Public Health, 2018; 15; 392. Angela Dawson, Learning and curriculum design in community health nurse education, PhD Thesis, University of New South Wales, 2008.
} 
tropical regions and multiple sclerosis in northern climes that have to be considered when designing health education programmes. ${ }^{15}$ Of primary importance are the changing expectations of society that have occurred over time. A partnership in health care is now considered the norm. Many new initiatives have been introduced and employed to help to bring around the desired behavioural change in society as simply providing information alone is clearly proved to be inadequate. Among these new initiatives to change society's attitudes has been the application of nudge theory which creates an environment in which an individual will favour the desired outcome. ${ }^{16}$ Teams have been formed, often referred to as 'nudge teams' whose function is to employ behavioural insights to inform society along the lines of nudge theory. In parallel to this is the term behavioural economics which studies the effects of psychological, cognitive, emotional, cultural and social factors on the decisions individuals make. In global health terms, however, there are nations at different stages of development. This will constitute a need for a variety of approaches, with regard to health education and promotion techniques in these different settings, as the requirements will range from the most basic to the most sophisticated methods.

While the postage stamp serves to pre-pay mail, it is suggested here that it does possess a useful minor role in the delivery of health messages. However, it may have attributes that the major media used for health promotion lack. The stamp can be rapidly adapted to meet the health and cultural needs of individual countries. It is exactly this cultural adaptive ability that has been stressed as important for postage stamps emanating from Africa. ${ }^{17}$ Recent publications have pointed to the fact that postage stamps are a primary information source, but they still remain a neglected medium for communication and provide a rich field for research. ${ }^{18}$

The aim of this study is to determine just how useful postage stamps have been, over time, in health promotion and education by posing the following research question:

\footnotetext{
${ }^{15}$ MSR, Hutt, DP., Burkitt, The geography of non-infectious disease, Oxford: Oxford University Press, 1986.

${ }^{16}$ Chris Perry, Krishna Chhatralia, Dom Damesick et al., Behavioural insights in health care, London: The Health Foundation, 2015.

${ }^{17}$ Posnansky, M., “Propaganda for millions, images from Africa”, African Arts, 2004; 37: 53-57.

18 Yehiel Limor, Ilan Tamir, "The neglected medium: postage stamps as mass media", Communication Theory; 2020: March; 1-15. https://doi.org/10.1093/ct/qtz043 accessed 26/6/2020.
} 
How and by what means have postage stamps and postal material played a part in health promotion and health education?

There is an ever-expanding number of postal authorities that issue postage stamps. This coupled with the fact that there are a large number of health issues that exist, as well as new disease entities recognised, requires a selection to be made in the health topics to be addressed in this study. The selection of topics in turn, would be dictated by the health developmental status of the nation, the relevance of the health education or promotion topic examined, and above all, an adequate number of stamp issues existed related to the selected topic. Thus, high-income and low-income countries may have employed stamps that are more suitable to their specific needs and life-style related conditions. ${ }^{19}$ The postage stamp, itself, is now established and recognised as primary source material for research purposes. ${ }^{20} \mathrm{It}$ is estimated that some 250,000 different stamp designs have been produced since 1840, giving rise to over 500,000 individual stamps. At current rates, 5,000 new designs are being produced annually. ${ }^{21}$ This research project is not related, however, to postal history or methods of stamp production, but to the secondary function of the postage stamp where its use is contingent on its postal function. This secondary function is one of delivering a message whether it is one of political propaganda or the visual or historical attributes of the issuing country or its educational needs.

The final format of this presentation consists of an introduction and a methods section, and specific chapters devoted to the postage stamp as a miniature messenger, with examples of its use as a visual medium, and one devoted to the definition of a health stamp in relation to health promotion and education. Further chapters are related to the selected topics with specific chapters on malaria, smoking, immunisation, diet and nutrition. An additional

\footnotetext{
${ }^{19}$ Leading health issues often targeted in high income countries include, gambling, mental illness, smoking, alcohol and drugs, immunisation, sun safety, heart disease, diabetes and workplace safety. In low income countries targets are often, malaria, malnutrition, breast feeding, immunisation and hygiene. Health Promotion Agency (NZ).

https://www.healthed.govt.nz/search?topic\%5B0\%5D=48\&type=resource\&mode=picture-view accessed $6 / 5 / 2020$.

${ }^{20}$ Charles Ekker, "Stamps as a unique primary research materials", Topic Time, 1969; 20.5: 40-41. Jack Child, The semiotics and politics of Latin American stamps, Durham: Duke University Press, 2008:5.

${ }^{21}$ Richard L. Sine, "Stamps the alphabetical issues from 'A' to 'D'”., The New York Times, 3 February 1985. Peter Elias, How many postage stamps are there in the world? http://www.quora.com/Peter-Elias-7 accessed 7/5/2020.
} 
chapter is devoted to analysing the position of the postage stamp in the overall scheme of health promotion, past and present. The discussion and conclusion should provide primary information as to the part played by postage stamps in the delivery of health information, education and promotion and thus deliver an answer to the posed research question.

\section{Methodology}

The study design that was devised to address the research questions employed a secondary form of research technique with predominantly qualitative methodology, as exemplified by the use of illustrated case studies. A quantitative assessment was confined to defining what actually constitutes a 'health education or promotion' stamp.

An initial systematic literature review was conducted that included the terms "stamps and health", and "stamps and health promotion" and was conducted and directed over multiple databases that included Google Scholar, Ebsco, Embase and the Cochrane Library Reviews. The latter, for example, contained five articles on postage stamps and over 300 on health promotion, none of which related to the topics in hand. Once these were carried out, more refined searches were undertaken that included the websites of the United States National Library of Medicine at the National Institute of Health (PubMed) and the digital library of JStor. ${ }^{22}$ The refined searches were directed at specific disease entities, and their relationship with health and postage stamps e.g. malaria and health promotion. In addition, relevant articles were sought from the websites of the Smithsonian Institute National Postal Museum, The British Postal Museum, and the National Library of Australia via Trove. ${ }^{23}$ Priority was given to peer reviewed, high impact journal articles and open access URL articles were confined to those listed to academic sources or well established entities such as international newspapers e.g. New York Times.

A primary source data base was constructed from data drawn from several origins. The principal source was by reference to the postage stamp catalogues published by Stanley Gibbons Limited in the United Kingdom. ${ }^{24}$ These catalogues list over 500,000 postage

\footnotetext{
22 Digital library, https://www.jstor.org/ accessed from 9/5/2020

${ }^{23}$ The Smithsonian Institute's Postal Museum permits access for research into postal matters, https://postalmuseum.si.edu/ accessed 9/5/2020.

${ }^{24}$ Stanley Gibbons, Stamps of the world, Ringwood, England: Stanley Gibbons Ltd., 2017. Stanley Gibbons, Commonwealth and Empire stamps (1840-1970), Ringwood, England; Stanley Gibbons Ltd., 2017 and United States of America, 2010.
} 
stamps that have been published worldwide, and provide information relating to the date of issue, the topic or theme of the stamp, and include a small image of the particular stamp or one of a stamp series, often in colour. A numbering system is included for ease of reference and identification. However, not all postally relevant material was listed in these catalogues, such as postal cancellations and specially designed postal covers as demonstrated in figure 1. Specific searches were then made to identify these additional appropriate philatelic materials, viz postage stamps, postal cancellations, first day of release envelopes, and postal labels. Searches were made for relevant material on stamp dealers' sale lists, the eBay web site and in philatelic journals. ${ }^{25}$ Finally, a search was made on the relatively recent Universal Postal Union on-line site, the WNS system, that shows images of postage stamps released by individual countries since $2002,{ }^{26}$ Material indicating a health topic were added to the data base and included for later analysis for health education content. The major exclusion criteria were related to the carriage of a surcharge on stamps that were intended to raise funds for health-related purposes (charity stamps) but did not carry a health message or exhibit an image related to a health topic. Similarly, those stamps which celebrated a health organisation e.g. World Health Organisation or awareness of health organisation e.g. Red Cross, were excluded (see figure 2). Neither of these formats have been regarded as true health promotion material in this thesis and the basis of this decision is discussed in detail later. However, a few exception were included and are justified in the text.

Once the target primary data was identified, efforts were made to obtain examples of each type of stamp from sources such as philatelic dealers, stamp fairs and from online sources such as eBay and Trade Me. Each stamp obtained was scanned using a Brother MFC9340CDW laser scanner and subsequently digitally manipulated to form a jpeg image by using Photoshop Elements 7 (Adobe Systems). This archival material was supplemented by additional and appropriate photographs, first day covers and relevant material and their source was cited as and when necessary and appropriate.

\footnotetext{
${ }^{25}$ Gibbons Stamp Monthly and Linn's Stamp News, the latter has the world's largest circulation for a philatelic Journal offering news and research material.

${ }^{26}$ Universal Postal Union, https://www.wnsstamps.post/en/WNS-Stamps/Stamps-Search accessed $15 / 10 / 2020$.
} 


\section{SG UNITED}

\#herewithyou

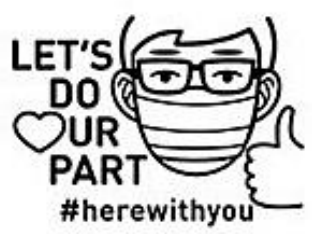

STAY HOME.

PROTECT THE NHS.

SAVE LIVES.

Royal Mail - keeping

communities connected
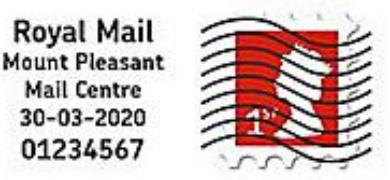

Figure 1. Stamp cancellations from Singapore and United Kingdom with health messages relating to the Covid 19 pandemic, 2020.

The original searches revealed a vast number and range of available stamps with health messages that were directed at a wide range of diseases and life-style health problems. For example, the small country of Cuba, produced 74 postage stamps with a health topic between 1933 and 2000. These topics ranged from tuberculosis to yellow fever and childcare. From these findings, from a practical and realistic aspects, and also the word limitation permitted, a selection of relevant topics had to be made. The selection made was based to reflect the cultural, social and climatic aspects that may impact upon health and disease prevention and hence the areas of infectious disease, immunisation, life-style induced disease, diet and nutrition were chosen.

To support the analysis, and to illustrate the arguments put forward in this dissertation required a large amount of relevant image material to be presented. The whole strategy behind the use of postage stamps in health promotion does focus upon the power of visual imagery with the verbal message (if any) taking a secondary role and hence the requirement to present many illustrations. ${ }^{27}$ This aspect of visual imagery is developed in a specific chapter. On occasions, a photographic image of a stamp topic was included so a judgement can be made as to whether the depiction is a true representation of the topic or message portrayed by the stamp. The stamps are regarded as primary source material and therefore their presentation, imagery and description act as their own literature review.

\footnotetext{
${ }^{27}$ David Isaacs, "Power of visual imagery", Journal of Paediatrics and Child Health, 2016; 52: 859-860.
} 

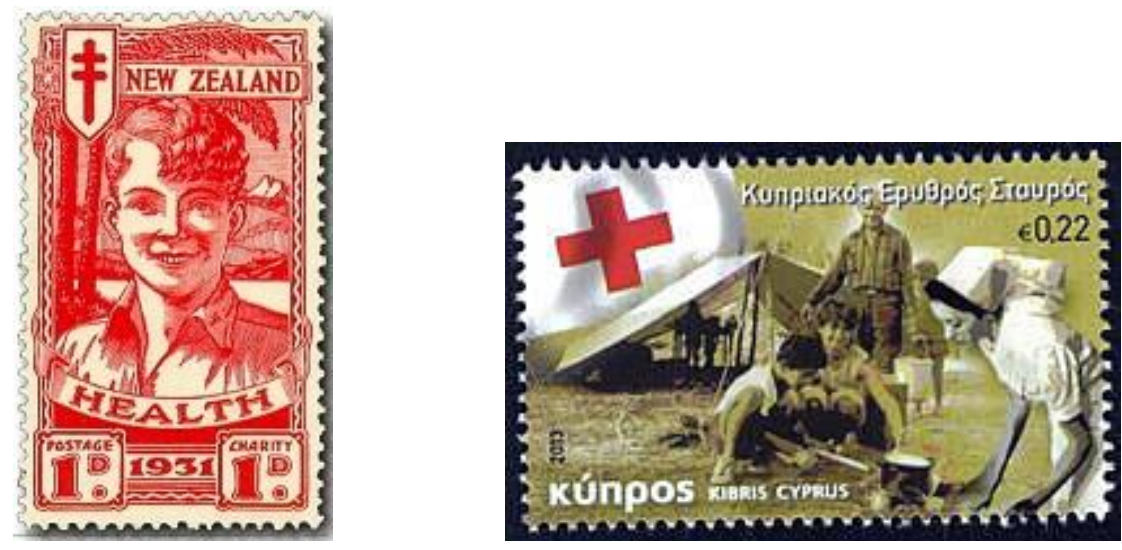

Figure 2. Examples of health charity stamp, New Zealand, 1931 and Red Cross celebration stamp, Cyprus, $2013 .^{28}$

The information collected in the manner described above was treated as qualitative data and was to be subjected to content analysis. ${ }^{29}$ The nature and difficulties of this type of analysis are discussed later. However, the analysis was to be dependent upon the reading of visual materials rather than text, and an appropriate method to do this had to be sought. In prior research, Gillian Rose, had described methods to read visual materials such as advertisements, museum displays and photographs. ${ }^{30}$ However, this work did not include reading visual materials such as postage stamps. One of the problems to be faced with context analysis of visually orientated material is that different individuals interpret images differently. ${ }^{31}$ Despite this, Raento and Brunn stated that "visual images do not exist in a vacuum" and there is a growing literature supporting the ability to analyse this type of material. $^{32}$

The context analysis used here is modelled on that of Raento and Brunn and used in several of their studies on postage stamps. Their method, itself, was also based upon the work of Lutz and Collins who described methods of 'reading' cultural images. ${ }^{33}$ This work is based

\footnotetext{
${ }^{28}$ For further discussion of New Zealand charity and health stamps, see Mark Stocker, "The 'Smiling Boy' health stamps of 1931", Tuhinga, 2013; 24: 139-158.

${ }^{29}$ Mariette Bengtsson, "How to plan and perform a qualitative study using content analysis", NursingPlus Open, 2016; 2: 8-14.

Hsiu-Fang Hsieh, Sarah E. Shannon, "Three approaches to qualitative content analysis", Quality Health Research, 2005; 15: 1277-88.

30 Gillian Rose, Visual methodologies: an introduction to the interpretation of visual materials, London: Sage, 2007.

31 Pauliina Raento, Stanley D. Brunn, “Visualizing Finland: postage stamps as political messengers”, Geografiska Annaler, 2005; 87: 146-163.

32 Ibid: 147.

Catherine A. Lutz, Jane L. Collins, Reading National Geographic, Chicago: University of Chicago Press,1993.

${ }^{33}$ Stanley D. Brunn, "Stamps as messengers of political transition", The Geographic Review, 2011; 101: $19-36$.
} 
upon the deconstruction of stamps because they convey multiple meanings. In reality the pre-selection of stamps for this study was ruled by a dominant single theme - health education and promotion - and this aided selection of the material. The context analysis is based upon the principles and key questions outline by Raento and Brunn viz. 1. How are particular images (or words) given meaning? 2. Are there meaningful clusters of images? 3. What associations are established within such clusters? 4 . What associations are there between clusters? 5 . What was not there? Invisibility may be relevant.

Further information to be sought to aid in the analysis are questions such as 1 . Has there been a transition in stamp themes over time? 2. Has there been widespread use internationally of stamps for health education? 3. Does the 'reading' of health orientated stamps give insight into the way they have been designed and adapted to give specific meaning for given health situations? 


\section{Chapter 1. The Miniature Messenger}

Sir Rowland Hill introduced the adhesive postage stamp into the United Kingdom in 1840 for the prepayment of postal charges. The new system became immediately successful and popular, and this led to the adoption of postage stamps by all the world's postal authorities by the beginning of the $20^{\text {th }}$ century. ${ }^{34}$

It soon became apparent that stamps could have an important secondary function. Many national governments were quick to realise their potential in terms of advertisement and propaganda. This led, in due course, to stamps designed to portray the scenic attractions, history and culture of their specific country (Figure 3). ${ }^{35}$ It was soon realised, by certain academics, that this secondary function deserved serious study and historians were among the first to appreciate their potential in the teaching of history. ${ }^{36}$ Subsequent academic publications relating to stamps have ranged from science and social studies to finance and business. There have been contributions in the medical field, often historical in nature, or

\footnotetext{
${ }^{34}$ The International Postal Union (UPU) was established by the Treaty of Bern in 1874, with its headquarters in Switzerland, and using French as its working language.

35 Pan American Union, "The Americas through their postage stamps", Bulletin of the Pan American Union, 1944; June: 356. Jack Child, 2008:3.

${ }^{36}$ Basil Reed, "Philately and the teaching of modern history", History, 1923; 7: 266-273. Donald M. Reid, "The symbolism of postage stamps: a source for the historian", The Journal of Contemporary History, 1984; 19: 223-249.
} 
specialty based..$^{37}$ This was, therefore, the first indications of the potential of the postage stamp in health education.
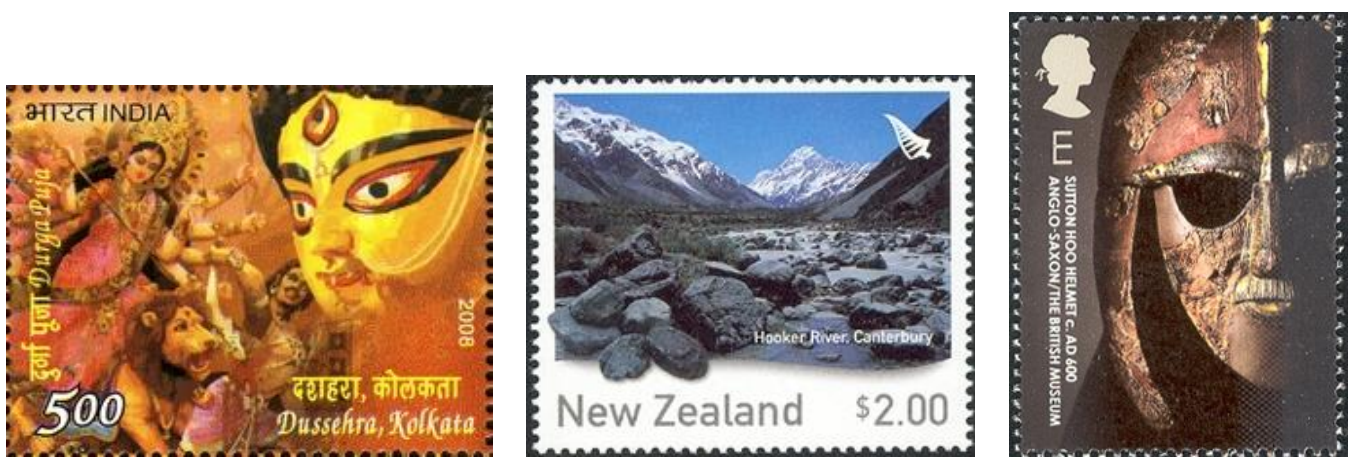

Figure 3. Examples of stamps depicting culture (India, 2008), scenic beauty (New Zealand, 2003), and history (Great Britain, 2003).

The American philosopher, Charles Pierce, was a pioneer in the development of the discipline now called semiotics - the study of signs and symbols and the messages they can contain. Pierce devised the concept of three basic classes of typology, viz 'icons' 'indices', and 'symbols' and further described many subdivisions off these classes. ${ }^{38}$ David Scott applied Pierce's theories to postage stamp design. His semiotic approach showed that as far as the primary function of the stamp was concerned, the 'icon' could be landscape (as in Swiss or New Zealand stamps, see figure 3). The 'index' is the pointer sign as to the country of origin - a printed map or sovereign's head (see figure 3, British stamp) - and the 'symbol' represents the numbers or letters of value. However, when it comes to the secondary

\footnotetext{
${ }^{37}$ Albert E. Chudley, James C. Haworth, "Genetic landmarks through philately - haemophilia", Clinical Genetics 1999; 56: 279-281. John Pearn, "Paediatrica philatelica", Journal of Paediatrics and Child Health, 1999; 35: 232-236.

JStor lists over 1300 academic papers relating to stamps as of 2020.

${ }^{38}$ Charles S. Pierce, Pierce on signs: writings on semiotic, ed. James Hooper, Chapel Hill: University of North Carolina press, 1991.
} 
function of a stamp, the 'symbol' represents the message, commemoration or celebration. ${ }^{39}$ It is this secondary symbolic function that we are concerned with here. Many themes, such as health, can often be represented by metonyms, which are partial representations of the whole image, as is seen in the frontispiece (qv), where the cigarette represents the act of smoking. One other parameter of stamp production that needs to be stressed is the importance of the distinction between a 'definitive' stamp and a 'commemorative' stamp. The former portrays general iconic messages, and these are not time sensitive as they are issued for mail carriage over an extended time. Commemoratives, on the other hand, are issued for a specific purpose such as an historical event or as propaganda and tend to be time limited and sold over a short period. Health promotion stamps are commemorative in type.

What properties or aspects of postage stamp could make it a successful deliverer of health messages? Carlos Stoetzer has written extensively about the attributes that he considers that make the postage stamp a useful propaganda agent. ${ }^{40}$ He states "The stamp goes from hand to hand, town to town and reaches the farthest corner of a country or even the world and by the use of slogans and /or pictures can convey a message far and wide". He adds the rider that in order to be successful, the nominal value must not be too high and correspond to the current postal rates. It should not have an added surcharge for fund raising and be aesthetically pleasing and its message clear. ${ }^{41}$ Harlan Strauss has argued that propaganda stamps also work at the subliminal level as the message is frequently reinforced each time the stamp is encountered, as they work through a visual medium. ${ }^{42}$ The improved printing techniques that have evolved in recent years, the use of multiple colours and advanced design features have now all been incorporated into postage stamp production. Postage stamps have now the potential for even greater visual impact.

\footnotetext{
39 David Scott, European stamp design: a semiotic approach to designing messages, London: Academy Editions, 1995:198. Jack Child, "The politics and semiotics of the smallest icons of popular culture: Latin American postage stamps", Latin American Research Review, 2005; 40: 108-137.

${ }^{40}$ In this context, propaganda is defined as an organised programme of information on a selected topic.

${ }^{41}$ Carlos Stoetzer, Postage stamps as propaganda, Washington: Public Affairs Press, 1953: 1.

${ }^{42}$ Harlan Strauss, Politics, psychology and the postage stamp, State College, Pennsylvania: Forty-first American Philatelic Congress Book, American Philatelic Society, 1975; 162: 178-179.
} 
There is strong evidence that postage stamps do have a major impact and have featured in serious political events. ${ }^{43}$ Until recent times, postage stamps were regarded as the 'carriers' of the national image when governments had a monopoly over the postal services. Hence, stamps that are issued from a country are regarded as representing that country's views. The long-standing border dispute between Nicaragua and Honduras came to a head following the issue of a stamp suggesting the border dispute had not been resolved. Riots ensued and deaths and injuries resulted. Honduras issued stamps to rebut the claim (figure 4). A similar situation occurred between Bolivia and Paraguay during the war fought over the border Chaco region. Each country issued a stamp indicating that the Chaco belonged to them. The ensuing situation was summed up by the New York Times as "The war between Paraguay and Bolivia was not caused by stamps, but stamps with maps did grievously aggravate the situation". ${ }^{44}$ As recently as 2004 , three South Korean stamps claiming that Dokdo Island (Takeshima) was Korean caused such a furore in Japan (who also claimed the island) that the situation became so serious that the United States Secretary of State, Hilary Clinton became involved, however, the dispute slowly cooled thereafter but was not resolved (figure 4).$^{45}$ Earlier, during the first World War, the potent propaganda value of the stamp was utilised by both the Allies and Central Powers. As territory was occupied, the practice was to overprint the national stamp with the occupied country's name or viceversa. This was clearly intended as propaganda.
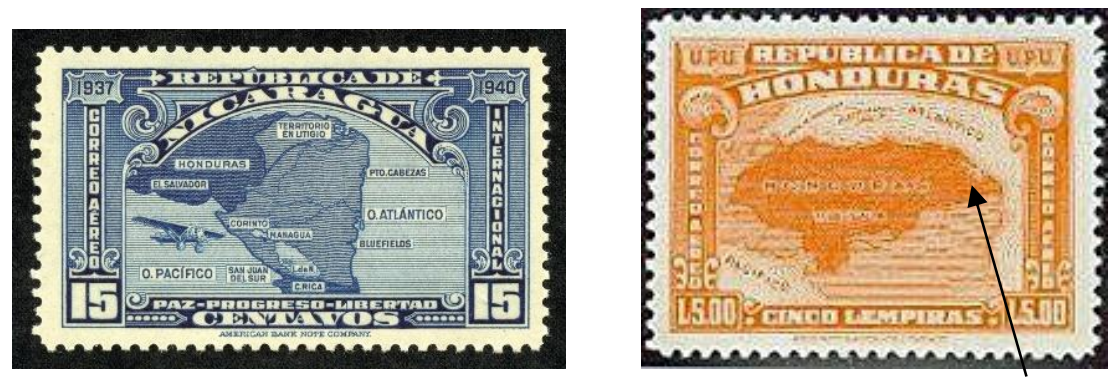

\footnotetext{
${ }^{43}$ Kenneth Dawson, Disputes over small territories, MA Thesis in Geography, Massey University, 2016.

44 John White, "Chaco foes remain at odds", New York Times, 26 September 1937.

${ }^{45}$ Sean Fern, "Tokdo or Takeshema?" Stanford Journal of East Asian News, 2005; 5: 78-83.
} 


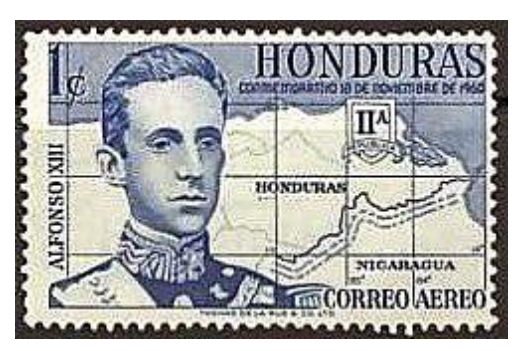

The offending Nicaraguan stamp of 1937, the Honduran stamp of 1943 with the arrow indicating the disputed area within their jurisdiction and the Honduran issue of 1960 issue after the boundary settlement.

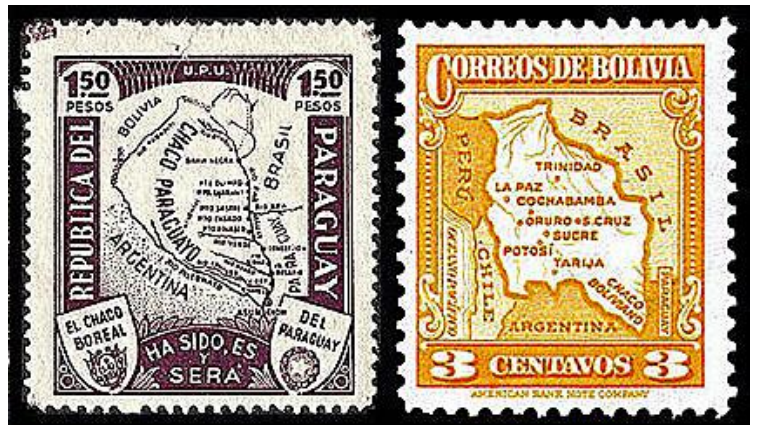

The rival stamp issues of Bolivia (1928) and Paraguay both claiming the Chaco which exacerbated the El Chaco war, which was eventually won by Paraguay (1932).
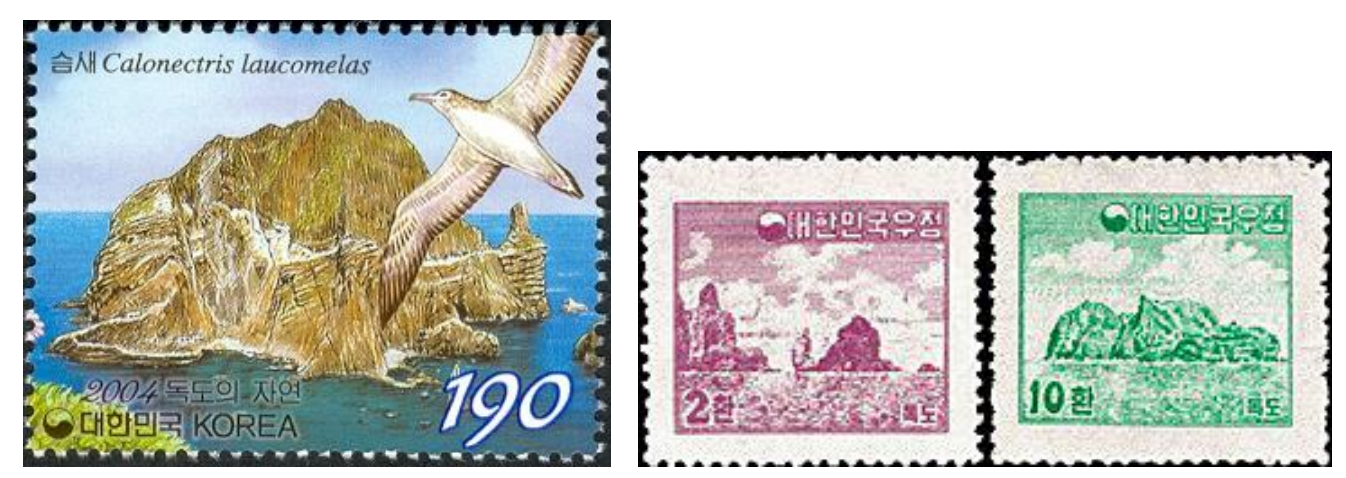

Figure 4. One of the 2004 issue from South Korea that caused an international incident, and two the 1954 issue which also pointed to the island's ownership by South Korea and not Japan.

as, for example, the parts of France occupied by the Germans was unlikely to engender much local mail when a military post existed. Figure 5 illustrates examples of this early use of the stamp to convey messages to the opposition. 

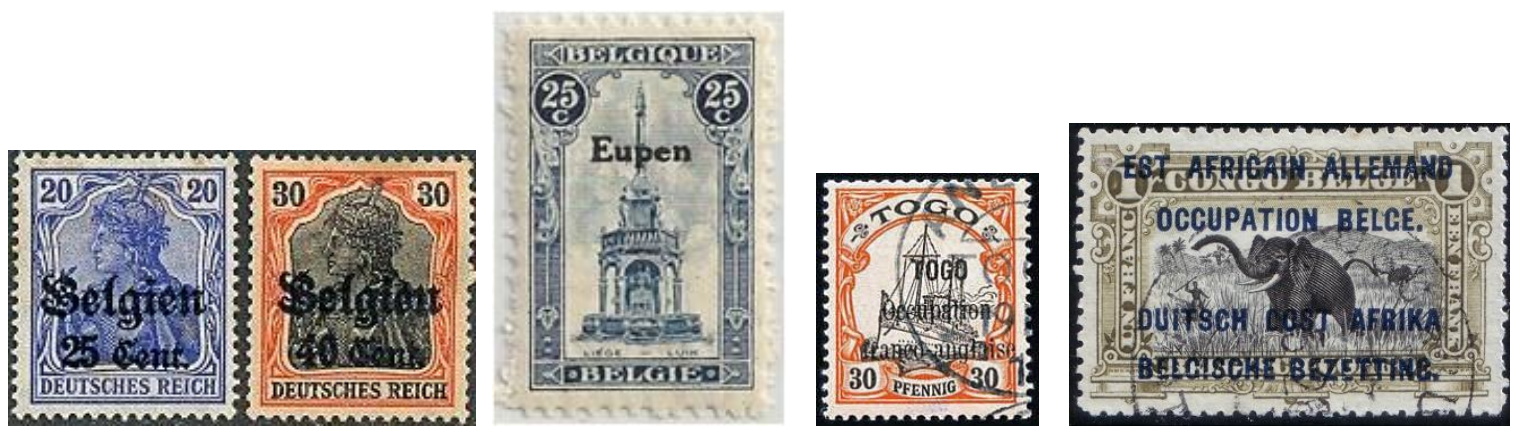

Figure 5. Examples of First World War, occupation overprints, German occupation of Belgium, Belgian occupation of Eupen (then German), Anglo-French occupation of Togo, and Belgian occupation of part of German East Africa.

By the time of the Second World War, the development of the stamp, as a potent force for propaganda, had reached a fine art. Stamp forgery were introduced to undermine the financial system of the postal authorities and also to act as agents for espionage. In addition, pseudo-stamps were produced with the intension of undermining the state and parodying its leaders (figure 6). ${ }^{46}$

To this point, this chapter has outlined the development of a secondary function for the postage stamp. This secondary function can reach a point in which it can overshadow the primary function as a postal receipt when it becomes a propaganda stamp for national advertising for tourism, politics or education. In other words, the 'primary' function is simply a way of delivering the message. When the stamp is used for political reasons it clearly can have a major impact as demonstrated by the events in South America and Asia.

As a starting point, it has to be determined what constitutes a health education and health promotion postage stamp, as opposed to a simple postal label, before an assessment of its use and value can be made. ${ }^{47}$ There are certain criteria that must be met to justify the title of a postage stamp and these are laid down by the Universal Postal Union and all approved new stamps appear on their website. ${ }^{48}$ First, it must meet the postal requirements of its primary function (see figure 7). The stamp requires to have a denomination or an indicator

\footnotetext{
${ }^{46}$ Herbert A. Friedman, https://www.psywar.org/stamps.php accessed 19/6/2020.

${ }^{47}$ The best know labels are the Christmas postal seals used for the raising of funds for tuberculosis treatment. These were introduced in 1904 by their Danish inventor, Einar Holbøll, and continue to this day in varying Formats.

48 Universal Postal Union, https://www.wnsstamps.post/en accessed 16/9/2020.
} 
its postal rate, the country of origin or a recognised symbol of its source. ${ }^{49}$ The design can simply be a number or a head of state's symbol, but for propaganda stamps the image is obviously paramount. There may be textual elements, but these are not essential, but may augment the health message and support the design. The health education stamp's visual message or graphic design is the key factor in delivering the health message. The design has to take into consideration the small canvas available to it and thus considerable expertise is required of the stamp designer to produce a successful outcome. ${ }^{50}$ Additionally, there is some design scope in the shape of the stamp.
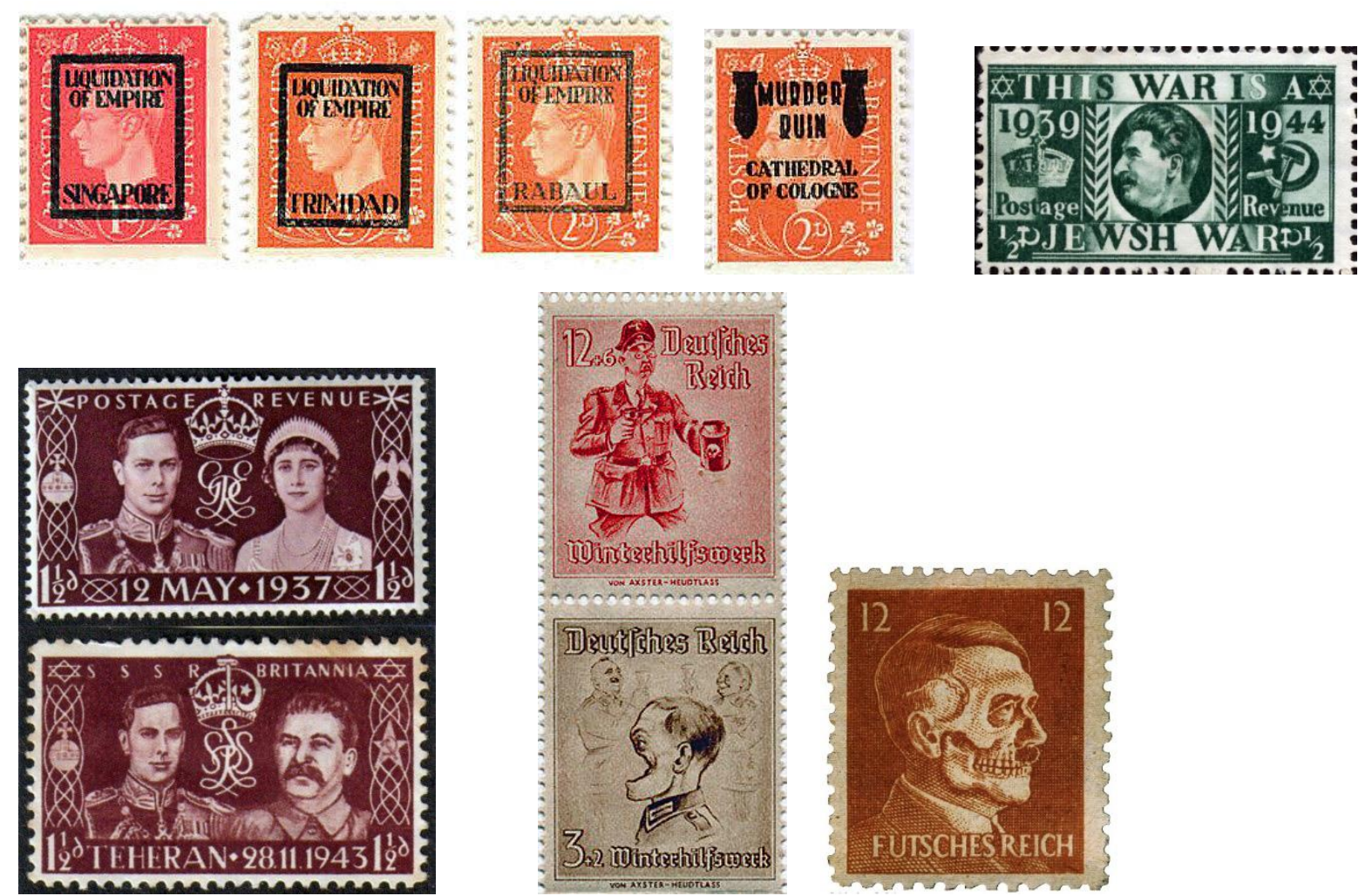

Figure 6. German propaganda stamps indicating the end of the British Empire, outrage at the destruction of Cologne Cathedral, and the influence of communism and Jews on Britain. British response to the war winter fund stamps inferring the funds are used for armaments and a skullcap parody of Hitler, entitled "Ruined Reich".

${ }^{49}$ Symbols such as " $\mathrm{E}$ " indicating postage for Europe only or "perpetual" indicating validity for changing postal rates. The United Kingdom stamps only carry the Queen's head to recognise that the UK was the first to issue stamps.

${ }^{50}$ The size of postage stamps so far produced has ranged from the smallest at $8 \times 9.5 \mathrm{~mm}$. to $210 \times 65 \mathrm{~mm}$. see James Mackay, The Guinness book of stamps, Middlesex: Guinness Publishing Ltd., 1988: 109-110. 
While most stamps are rectangular, successful designs have been based upon square, triangular, octagonal or even banana shaped formats. For general stamps, gimmick issues have been produced from plastic, gold foil or cloth. However, the overwhelming number of stamps are still on paper. Finally, the printing process can dictate or influence design, most modern stamps are produced by the photogravure method with minimal text and vivid colours. Therefore, to be a successful health education stamp, the designer has to be able to translate a complicated verbal subject into visual terms. Drama is often employed as the means by which this is achieved (see figure 7). ${ }^{51}$

In the analysis of the information presented in this chapter, there is strong evidence that stamps produced for health education and promotion could likely have a good chance of having a positive effective. This is based upon the fact that graphic images and pictures, as spatial imagery, have been recognised and shown to be powerful agents in communicating ideas and messages in other fields. ${ }^{52}$ Indeed, the examples of political stamps presented here have had a major impact and effect in relationship to national boundary disputes. A further potential advantage of the health promotion stamps is that they are not tied to a specific nation, in terms of their message, and they can contribute to international health themes and campaigns. Further, with the ever-improving methods of stamp production and printing should help with the impact of health education stamps if the health message is well designed within the small space available. There is a tendency for stamp size to enlarge to accommodate the secondary function. ${ }^{53}$

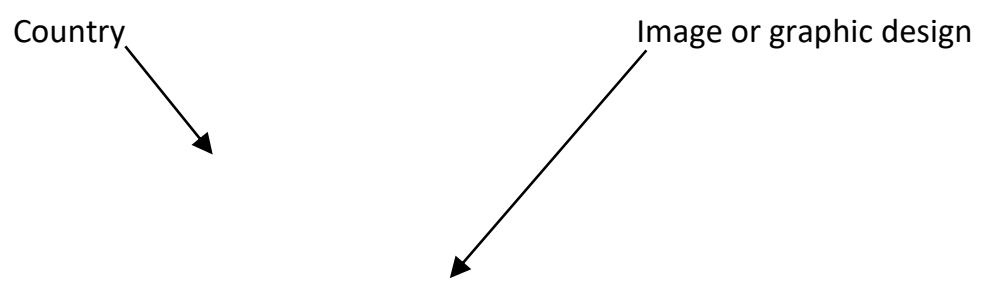

\footnotetext{
${ }^{51}$ David Gentleman, "The design and production of postage stamps", Journal of the Royal Society of Arts, 1974; 122: 431-449. Peter Jones, "Posting the future: British stamp design and the 'white heat' of a technological revolution", Journal of Design History, 2004; 17: 163-176.

52 Stanley D. Brunn, "Stamps as iconography: celebrating the independence of new European and Central Asian states", GeoJournal, 200; 52: 315-323, Pauliina Raento, Stanley D. Brunn, "Visualising Finland: postage stamps as political messengers", Geografika Annaler, Series B, Human Geography, 2005; 87: 145-163.

53 James Watson, The Stanley Gibbons book of stamps, Ringwood: Stanley Gibbons Publications Ltd., $1990: 74$.
} 


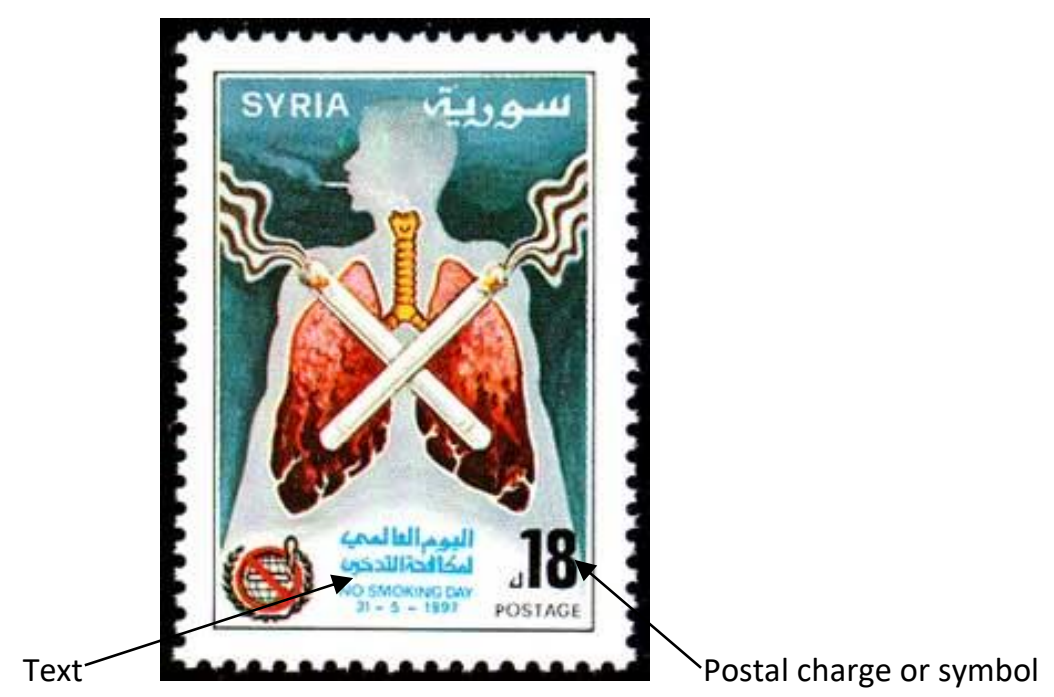

Figure 7. Basic requirements for a postage stamp.

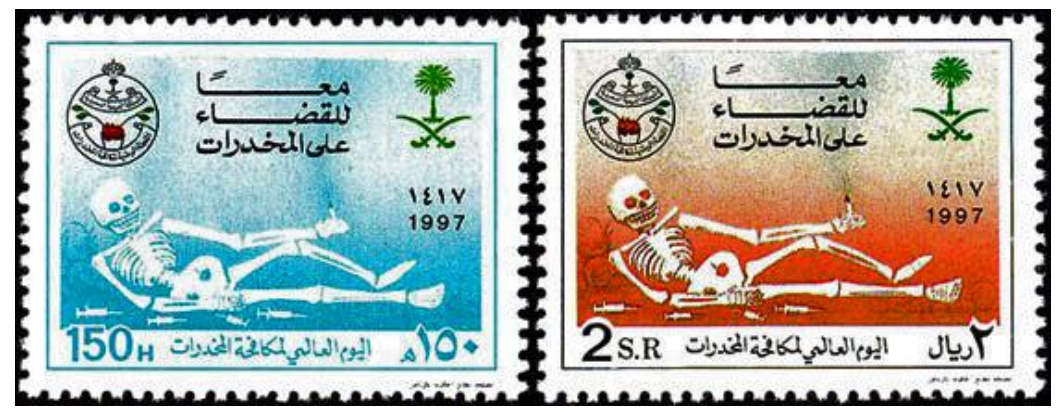

Figure 8. Saudi Arabia (1997), Anti-drugs campaign issue illustrating the danger of drugs.

There are two other important factors that need to be considered that could influence the success of health education stamps. There has been a steady fall in the number of letters posted worldwide and a rapid rise in email and metered mail usage. Child has argued that for reasons of nationalism, propaganda, and financial returns, postage stamps will continue to be produced. ${ }^{54}$ Perhaps to this opinion it should be added specifically, that health promotion material will continue as it has for over fifty years. The second matter that has been raised by Robert Greenwald relates to the target audience of the health message. He argues that many countries have a high illiteracy rate and that their stamp production is only intended for stamp collectors and may not even reach the putative country. ${ }^{55}$ However, the counter argument has been raised by Merrick Posnansky, in relation to Africa, where he makes the case that stamp images from Africa should be encouraged, with African designs

\footnotetext{
${ }^{54}$ Child, Semiotics, 2008: 196.

${ }^{55}$ Robert A. Greenwald, "The postage stamp as a messenger", Tobacco Control, 1992; 1: 87-88.
} 
and African designers, and countries should foster their stamps and form national collections. ${ }^{56}$ Perhaps the whole point that Greenwald misses is that in the cleverly designed postage stamp the image presented overcomes the problem of literacy. Further, these stamps often become icons, themselves and apart from being placed upon letters, they form distinctive images in their own right and are widely displayed. This is typified by the health stamps of New Zealand whose images are found on a range of articles from post cards to tea towels.

\title{
Chapter 2.
}

\section{A New Role for the Postage Stamp: a Health Messenger, Delivering a Textual Message in the Promotion of Health}

\section{Chapter 2.1}

\author{
The Health Messenger
}

The term 'health stamp' is a philatelic term and is used almost synonymously with the similarly named stamps of New Zealand that were issued between $1929-2016 .{ }^{57}$ This term is a misnomer as these stamps are charity stamps and are also termed 'semi-postal' issues in philatelic terminology. Apart from two issues, they do not carry a health message or a health related image, and are solely intended to raise money for a health charity such as

\footnotetext{
${ }^{56}$ Merrick Posnansky, "Propaganda for millions, images from Africa”, African Arts, 2004; 37: 53-57.

${ }^{57}$ Richard Wolfe, It's in the post: the stories behind New Zealand stamps, Nelson: Craig Potton Publishing, 2010: 72-79. Mark Stocker, "The 'Smiling Boy' health stamps of 1931", Tuhinga, 2013; 24: 139-158.
} 
children's health camps. A surcharge is added to the postal rate, which then goes to the charity. The images they do carry are often related to a sport, animals or an outdoor scene or pastime. There are two important exceptions, the first issues $(1929,1930)$ had a brief message (see figure 9), while the 2015 stamps can be clearly interpreted as a health promotion issue. They carry a clear message about sun exposure and how to prevent damage, along with the raising of revenue. This would appear to be an excellent promotion in light of New Zealand's high incidence of skin cancer, among the highest in the world (figure 9). ${ }^{58}$ Ironically, at the time when a true health message was being to be presented the concept of charity stamps ceased and that year's issue proved to be the last New Zealand health stamp.
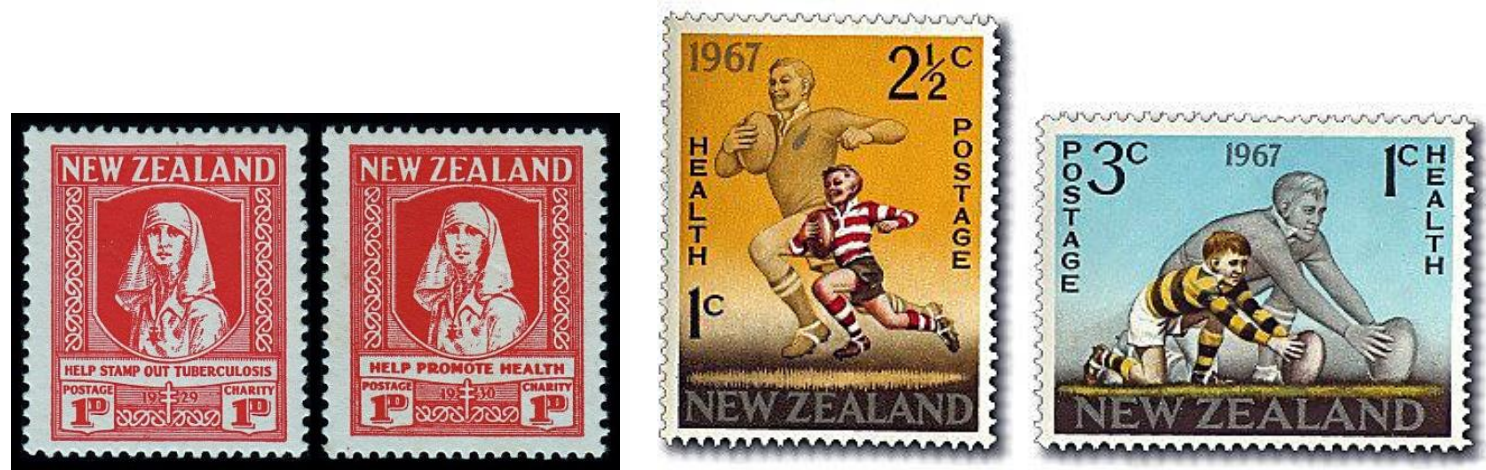

The first health stamps of New Zealand, 1929, 1930 carrying messages "Help stamp out tuberculosis" and "Help promote health". Both carry the Cross of Lorraine, the international symbol of the global fight against tuberculosis. The 1967 health stamp infers the playing of rugby is a healthy pastime.

\footnotetext{
${ }^{58} \mathrm{New}$ Zealand skin cancer rates, https://www.sciencelearn.org.nz/resources/1329-new-zealand-skin-cancerstatistics accessed 21/5/2010.
} 


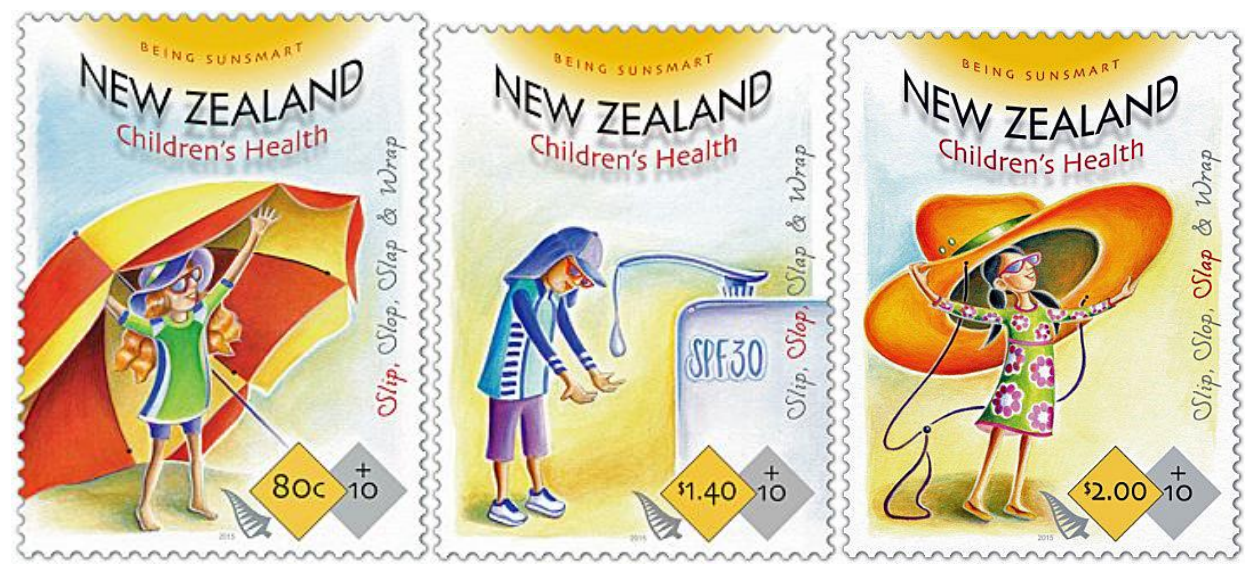

Figure 9. The 2015 New Zealand health stamps give clear instructions on how to avoid sun damage by being 'sunsmart' and are excellent examples of true health stamps.

It is now becoming apparent that there is not always a clear distinction between charity stamps and health promotion/education issues. This is particularly noticable in the many postage stamps related to tuberculosis. These have emanated from many countries around the world where tuberculosis was a major health problem and where the disease has shown an upsurge in recent years. The first of the tuberculosis-related stamps was issued by the Colony of New South Wales in 1897 and was followed by a Netherlands issue of 1906. Later the tuberculosis stamps carried the Cross of Lorraine symbol to distinguish them from other charity stamps (figure 10). ${ }^{59}$ On the borderland of being labelled prevention stamps, are those issues which carry an overprinted message advocating support against the disease. In addition, there are postal cancellations with tuberculosis messages (figure 11). A Belgian tuberculosis charity stamp is illustrated which has an additional anti-tuberculosis postal cancellation. Post cards were also released to encourage the purchase of the stamps intended to spread the message about tuberculosis and to raise funds for anti-tuberculosis organisations. An example of a French postcard promoting their purchase is illustrated in figure 12.

\footnotetext{
59 In 1902, Dr. Gilbert Sesiron in 1902, proposed that the Cross of Lorraine be chosen as the symbol of the global fight against tuberculosis and thereafter was accepted as the logo. The symbol itself dates from 800CE.
} 

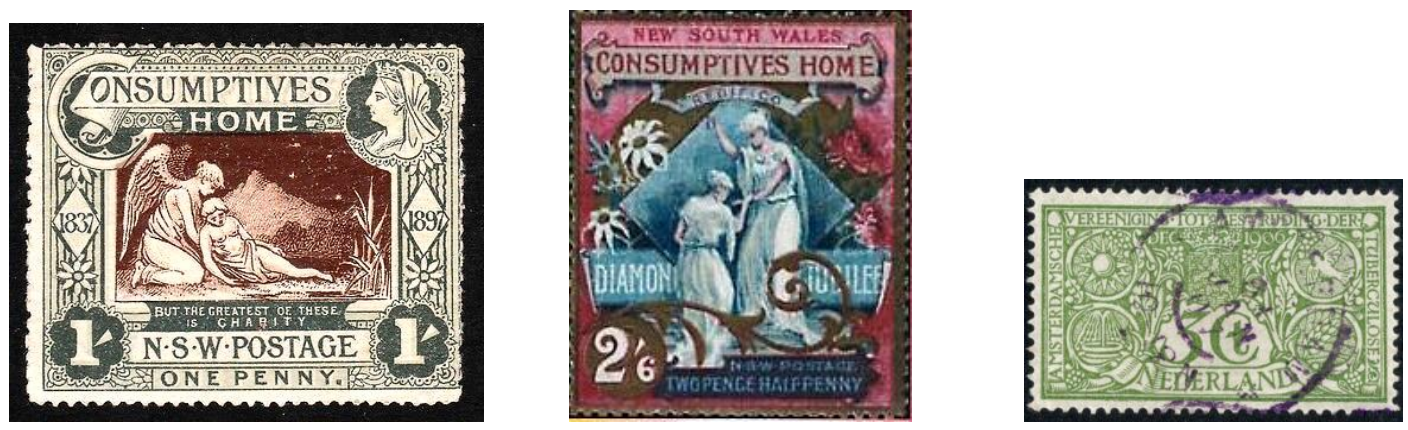

The earliest charity stamps for tuberculosis, New South Wales, 1897 and the Netherlands, 1906. There is no direct health message, but $1 /$ - and $2 / 6$ for charity was added respectively, on to the $1 \mathrm{~d}$ and $2 \mathrm{~d}$ postal rates in New South Wales at that time.
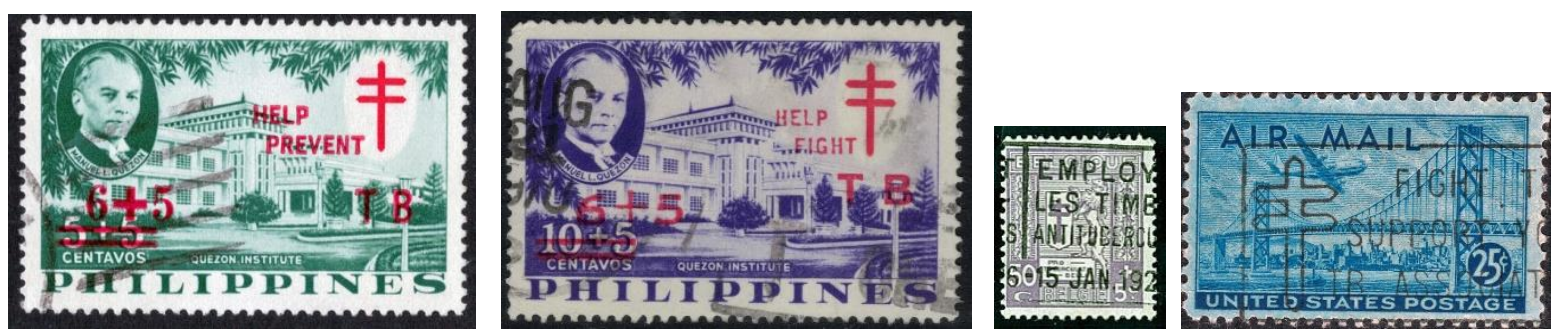

Figure 10. Philippines, 1959, tuberculosis related stamps with additional overprints - 'help prevent TB' and 'help fight TB', Belgian tuberculosis charity stamp with a postal cancellation ecouraging the use of antituberculosis stamps (1923). United States postal cancellation- 'Fight TB support your TB Association'on air mail stamp.
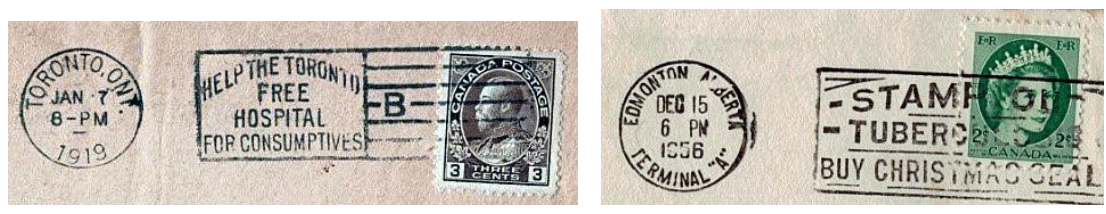

Figure 11. Canadian postal cancellations seeking support for tuberculosis charities, 1919 and 1956. 


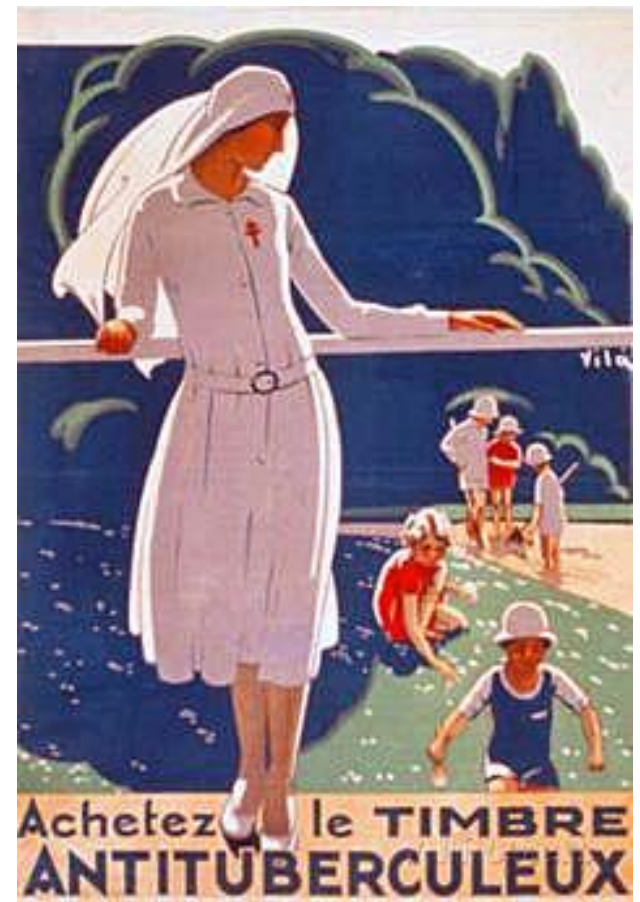

Figure 12. "Buy anti-tuberculosis stamps": source Pintrest.

In relation to tuberculosis there are more specific stamps that are more related to health education and intended to encourage the prevention of the disease by the use of B.C.G. (Bacille Calmette Guerin) immunisation. These are also educational as they illustrate the method of delivery of the immunisation, and are often produced as visual presentations only. Figure 13 is an example of a postal BCG promotion in one of the smaller Polynesian Island groups with clear representation as to what is involved. In contrast, the word 'BCG' only is used in a Caribbean promotion.

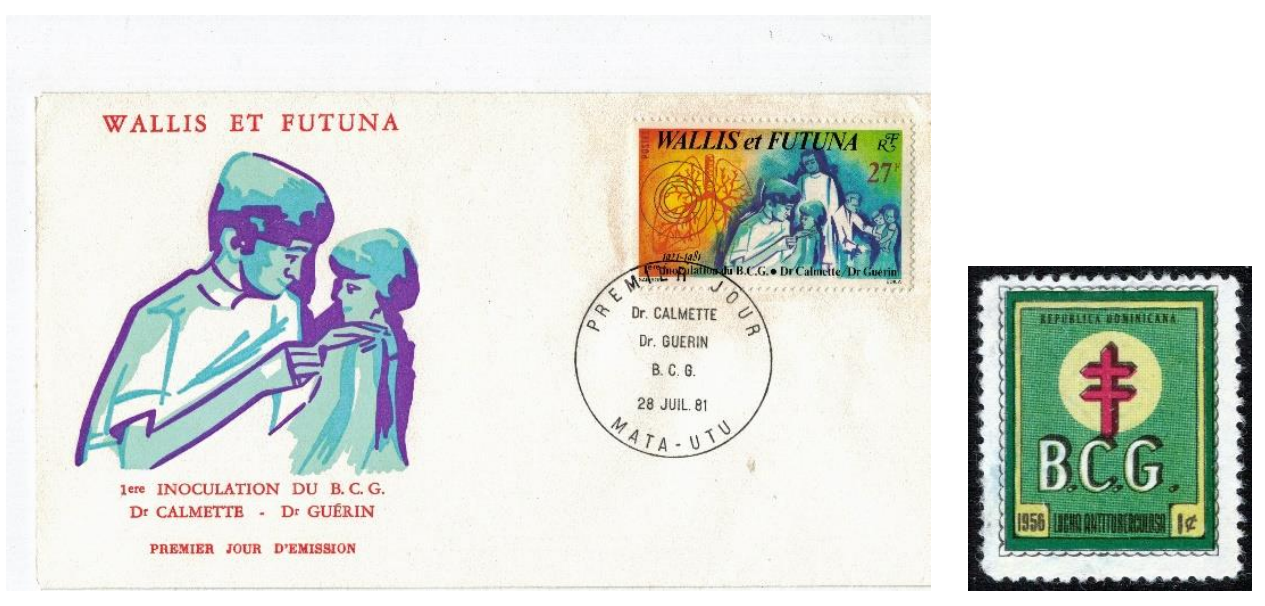

Figure 13. First day cover and stamp from Wallis and Futuna Islands (1981) encouraging BCG immunisation. Dominican Republic's visual association of the Cross of Lorraine with BCG. 
In a summary and analysis of this section, an important observation is that stamps that were issued as semi-postal stamps to raise money for health-related charities present, in general, a major lost opportunity to present a health education message in relation to the charity for which the money is to be raised. The obvious exception presented here to this criticism is the 'sun-smart' issue of New Zealand, which not only awareness of the dangers of sun exposure is demonstrated, but it gives information as to the prevention of sun damage. It is clear, thus, that some charity fund raising stamps do have a dual function and must be assessed when drawing conclusions about stamps and health education. Many other charity issues appear borderline and many anti-tuberculosis issues fit into this category. However, BCG tuberculosis issues do provide specific information and often fufil the dual role. Some of the charity presentations are more subtle and as shown in figure 14, the impact factor is related to the effect of the modification of the artist's painting into an anti-tuberculosis fund raising issue. However, in doing so, awareness of the disease is stressed by the picture and the written heading. The power of this presentation is the association of the mother and child with the symbol associated with the campaign against tuberculosis (the Cross of Lorraine).

Postage stamps have been issued covering a whole range of topics related to health and medical practice. These include the history of medicine, celebrated doctors and nurses and the practice. While interesting, these stamps have little relevance to health education and promotion. It could be argued, however, that issues promoting recent advances in medicine could be classed as health education. A recent example of this category of issue is that released by Australia in 2020, and shown in figure 15 . They may be helpful in focussing the population on health matters, but they do not promote health per se.

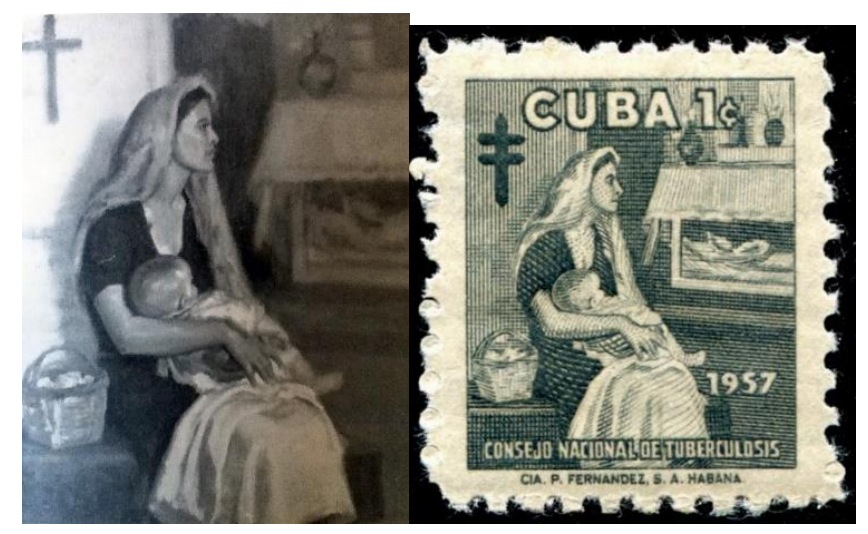


Figure 14. The Cuban artist Silvia Fernández Arrojo's original painting, entitled Refugio de Paz ("Refuge of Peace"), is the basis of the stamp design. It was issued by Cuba on November 1, 1957 to benefit tuberculosis (TB) funds. It is of note that there has been substitution, in the stamp's design, of the Christian cross in the painting by the anti-tuberculosis double-barred cross emblem (Cross of Lorraine). ${ }^{60}$

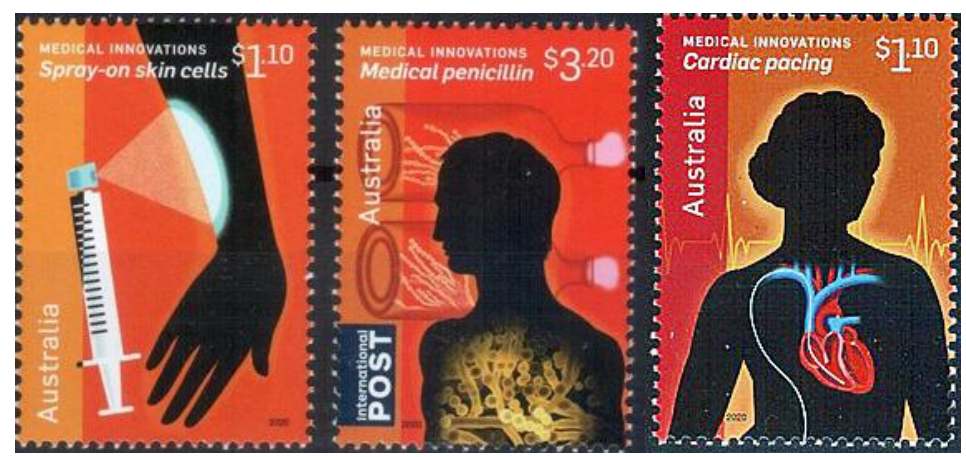

Figure 15. Australia, innovations in medicine series, 2020, skin cells, penicillin, and cardiac pacing.

\section{Chapter 2.2 Delivering a Textual Message}

While the visual stamp image usually predominates in the delivery of the health information, the written text can be supportive or even be essential to the overall presentation of the health message. Certain postal stationery items use the textual message in preference to the visual image. A good example of these are the Meghdoot postal cards issued by the Indian Postal Service that deliver a range of social and welfare messages, including health promotion, and still include the conventional postal card writing space (figure 16). ${ }^{61}$. India also issues standard postal cards with health messages as postal cancellations, an example relating to AIDS is displayed in figure 16 also. As can be seen in the figure the messages relate to health conditions relevant to India such as tuberculosis, malaria and AIDS. The specific Meghdoot card illustrated, stresses the importance of 'DOTS', directly observed treatment - short term, in the treatment of tuberculosis. This message is particularly important as India has a high incidence of drug resistant tuberculosis. The bilingual message (English and Hindi) is issued on the authority of the Ministry of Health and Welfare.

\footnotetext{
${ }^{60}$ Arrojo source: https://www.stampboards.com/viewtopic.php?f=17\&t=51327\&start=150 Accessed $24 / 5 / 2020$

${ }^{61}$ Meghdoot cards are named from the Sanskrit meaning 'cloud messenger' and are $14 \times 9 \mathrm{cms}$. in size. https://www.indianstampghar.com/category/philatelic-stationery/postalstationery/meghdoot-post-cards/ accessed 25/5/2020
} 


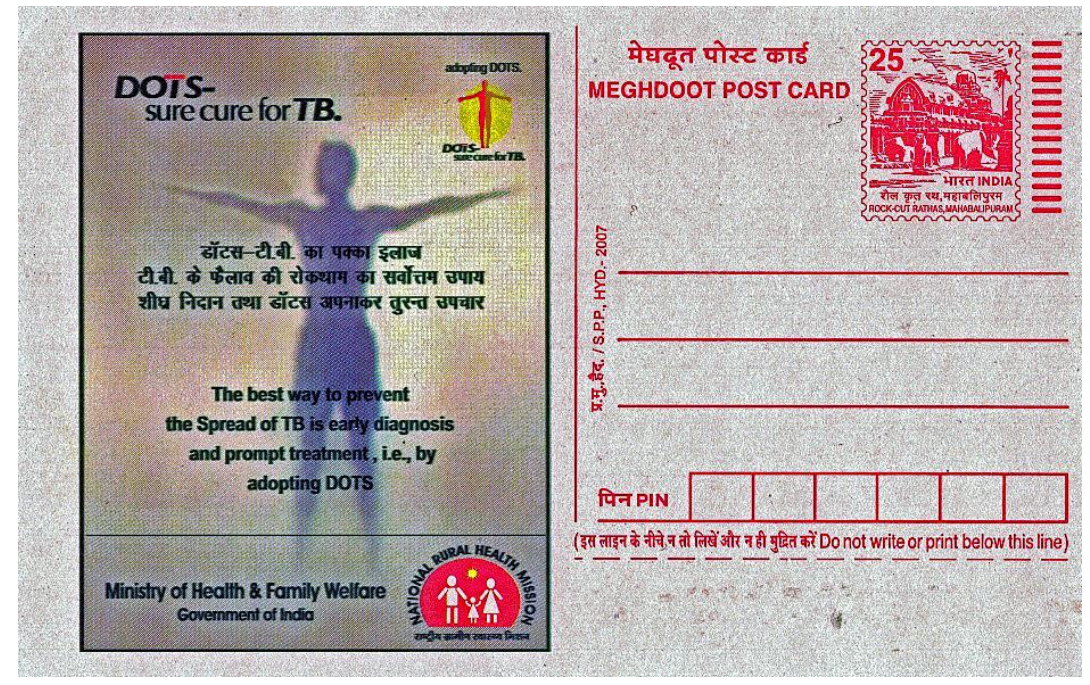

Meghdoot postal card, India, promoting DOTS treatment for tuberculosis.

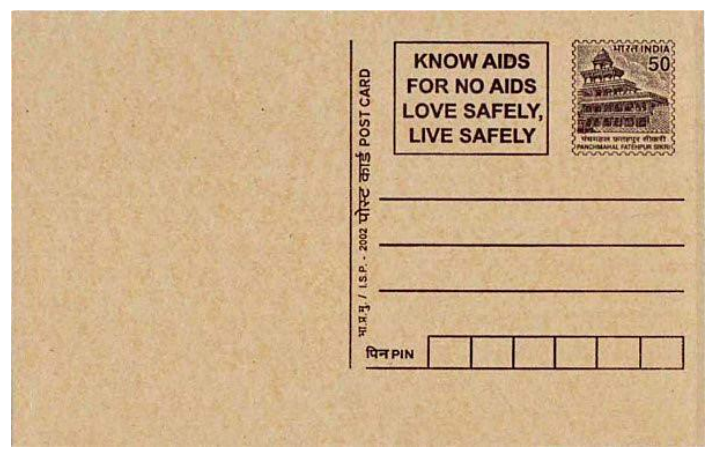

Figure 16. Conventional postcard, India, postal cancellation "Know Aids for no AIDS, love safely, live safely".

The wording on health-related stamps can lead to controversy. The United States issued two stamps related to breast cancer and one related to prostatic cancer in 1996, 1998 and 1999 respectively (see figure 17). Their release prompted an article in the prestigious New England Journal of Medicine criticising the wording on the stamps and commenting on health themed stamps in general. ${ }^{62}$ The authors argued that the text on the stamps was incorrect and that the prostate cancer stamp's wording "annual check-ups and tests" was fallacious. This comment was based upon the fact that there was no scientific evidence that the use of regular tests (prostate specific antigen) provided no reassurance and is not an accurate screening method. They stated that the text should state "Prostate cancer awareness" only. Conversely, they argue that the breast cancer stamp of 1998 should have a text that should support regular mammography screening as this does have scientific

\footnotetext{
62 Steven Woloshin, Lisa Schwartz, "The U.S. postal service and cancer screening - stamps of approval?", The New England Journal of Medicine, 1999; 340: 884-887.
} 
validity. The authors concluded that the U.S. postal authority should not publish healthrelated stamps unless they are first assessed and validated by reputable medical authorities. What actually happened subsequently, was that the breast cancer stamp of 1998 was not altered but was re-issued, in the same format as before, as a charity (semi-postal) stamp with 6 cents of the face value going to the breast cancer charity. At the time of this re-issue the following announcement was made:

The stamp art depicts a woman standing with her right arm raised, reaching behind her head in the position recommended for breast self-examination. The drawing of the woman's body is set against a background of pastel colours ranging from yellow to violet that cover the entire face of the stamp. Across the top of the stamp are the words "Breast Cancer." Circling the figure's right breast are the phrases, in all caps, "FUND THE FIGHT." and "FIND A CURE."63
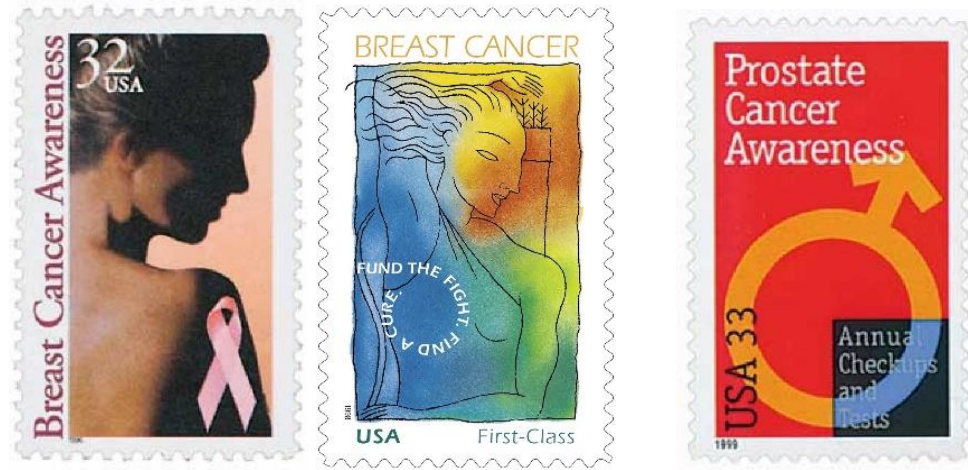

United States cancer stamps that resulted in controversy over their text, especially with the 1999 "Prostate Cancer Awareness" issue.

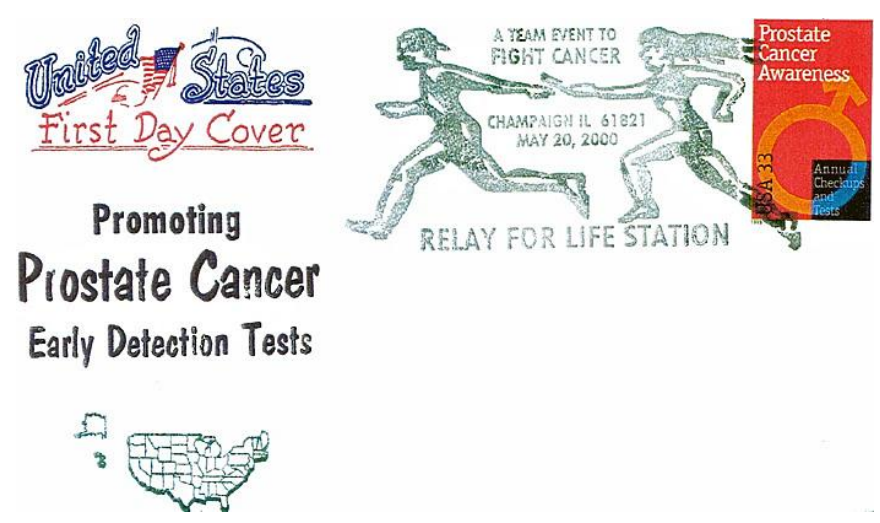

Figure 17. United States First Day cover re-enforcing the controversial prostatic cancer screening message.

${ }^{63}$ Breast cancer stamp: https://www.illustrationhistory.org/illustrations/breast-cancer-stamp accessed $25 / 5 / 2020$ 
Croatia produced a much clearer stamp to encourage breast screening as shown in figure 18. The text clearly states "mammography" in the appropriate language. There is no confusion here as this stamp does not state its relationship to breast self examination, as portayed in the United States stamp. The United States stamp offers no explanation and only uses the term "fight cancer". The Croatian stamp can be contrasted similarily with a equivalent Belgian issue which states "Fight against cancer", but shows a complex target and mammography picture, the former's message is precise and clear in contrast to the Belgian issue. Finally, it can be argued that the two stamps from South America in figure 18, while carrying visual impact, could have benefitted by having a text message relating to breast self-examination, importance and encouragement to learn the technique.

Despite the controversy over the United States cancer stamps, many short message texts have proved useful additions and have augmented the visual image. One way of achieving an increase in text space was first introduced by the Polish Post Office when they issued an anti-tuberculosis stamp in 1948. Attached to the postage stamp was an additional label promoting the importance of BCG in the prevention of tuberculosis (figure 19). More text may be achieved by having larger lettering imposed over or beside the image. Figure 20 shows examples of this method.

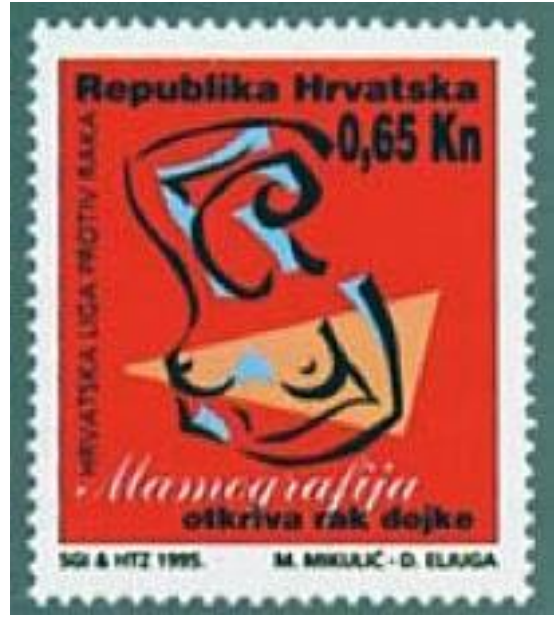

Croatia 1993, 'Mammography'.

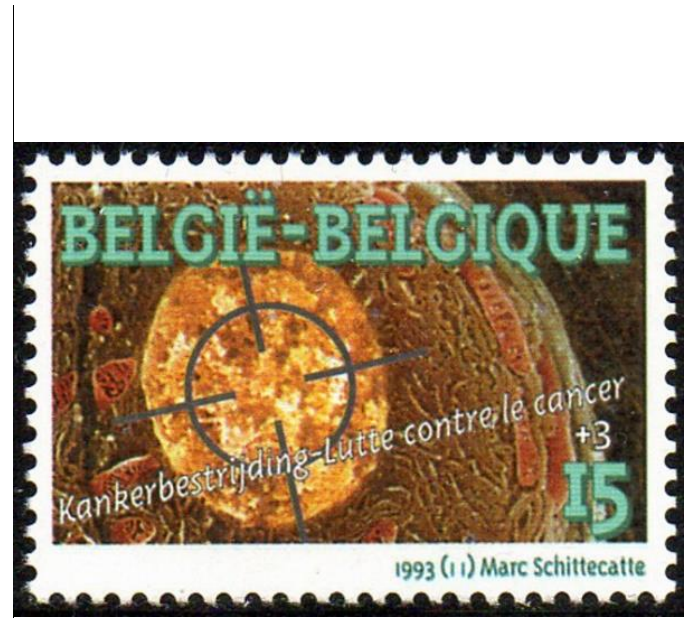

Belgium 1993, 'Fight against cancer'. 


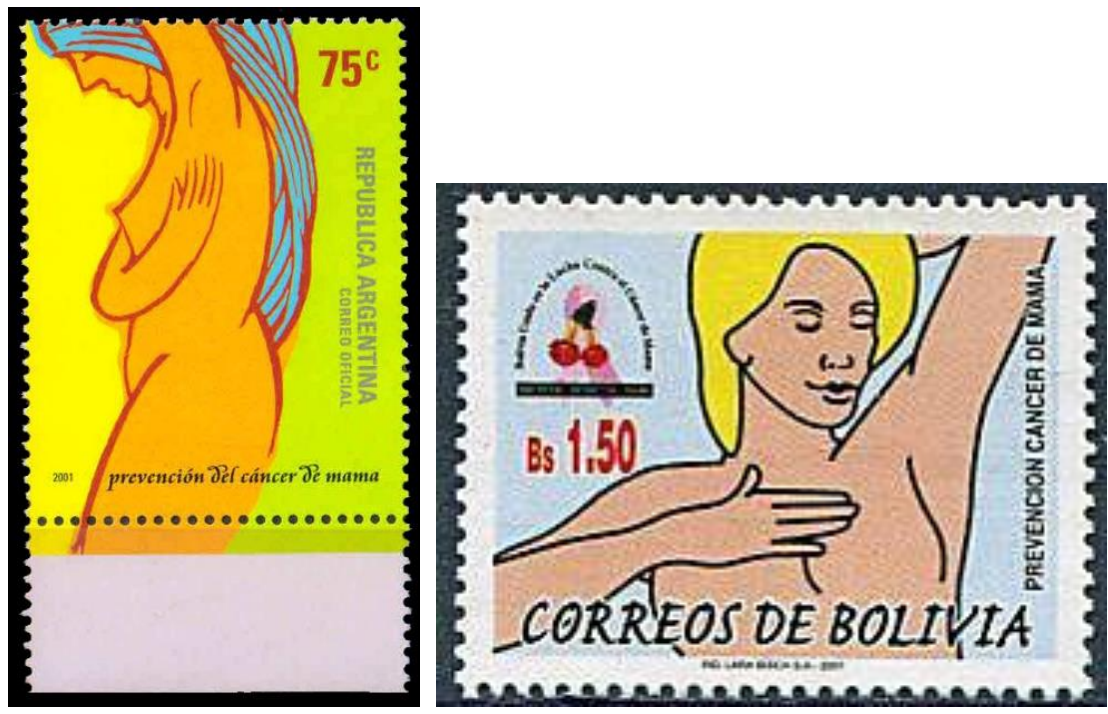

Figure 18. Stamps of Argentina and Bolivia (2001) issued for the 'prevention of breast cancer', there is no specific textual message about breast self-examination that the stamps illustrated actually demonstrate.
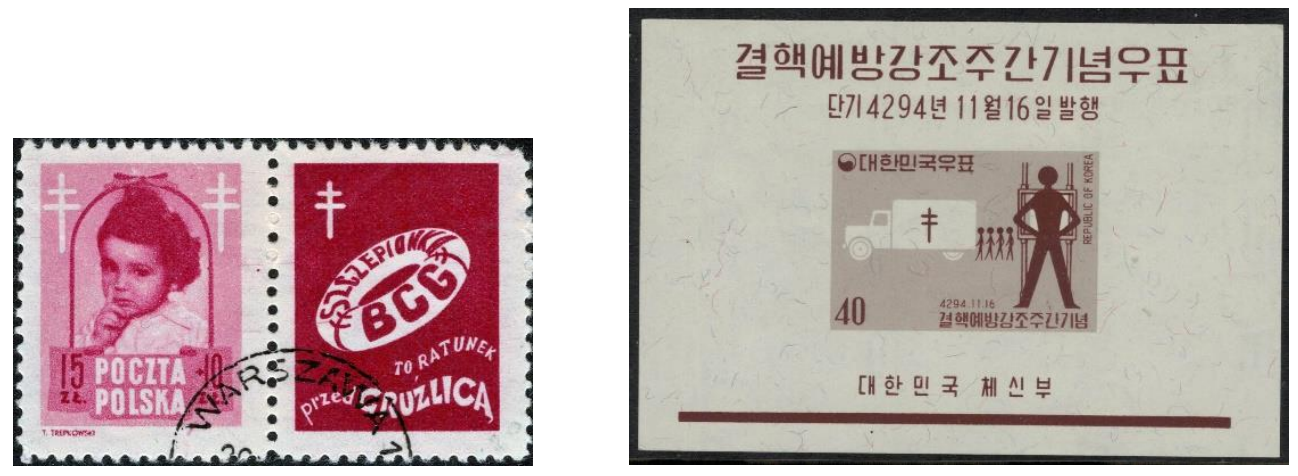

Figure 19. Early Polish stamp, with label attached, to promote BCG immunisation (1948). Postage is valid with or without label. South Korean mini sheet promoting mass miniature radiography (MMR) in an antituberculosis campaign (1961), the clear additional text is added to the basic stamp.
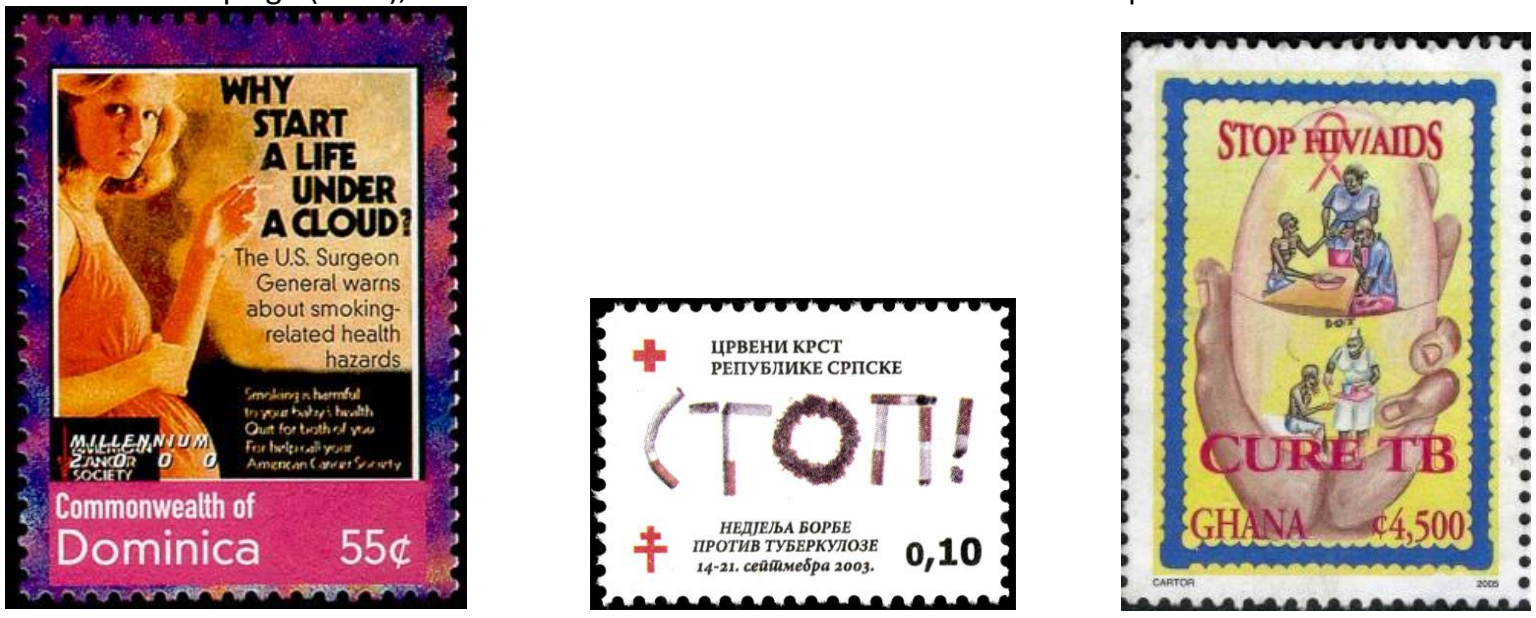

Figure 20. Three examples of text messages playing a major part in the overall message. Dominica's (2000) anti-smoking stamp, Bosnian stamp (2003) utilises the cyrillic letters to form the word 'stop' in relation to smoking. A direct statement about tuberculosis dominates the image in the Ghana stamp of 2000. 
It is now clear that a text message can add an important additional aspect to the stamp presentation. This is particularily effective in the stamp of Dominica (figure 20), where the use of a statement from the Surgeon General of the United States adds authenticity to the message that smoking is hazardous and one should not smoke and especially in pregnancy. Accuracy in the message, however, is paramount, and is well illustrated by the American prostate cancer stamp (figure 17) and the debate which followed. Much controversey followed the United States breast cancer issue and the suggestion that it presented a lost opportunity for health promotion. However, the decision was made re-authorise, adapt and reissue the stamp as a semi-postal charity stamp, something the United States had never done before. It required an Act of Congress in order to validate the production and sale and release of such a stamp. ${ }^{64}$ The outcome of all of this was that the stamp became the biggest selling comemmorative in United States history with over 900 million stamps sold and the sum of $\$ 50$ million dollars raised for breast cancer charity.${ }^{65}$ The controversey continued, however, and the academic Samantha King wrote: "Breast cancer has been transformed into a market drive industry rather than innovative ways of treating breast cancer" ${ }^{66} \mathrm{Be}$ that as it may, success was claimed by the supporters of the semi-postal fund in that it raised money that was used for a key breast cancer study which yielded important results. ${ }^{67}$ It can be argued that more important than health promotion or education was the acquisition of new practical treatment information. However, with the financial success of the stamp prompted Hungary to issue an identical stamp, the text in Hungarian, to raise funds for breast cancer there (figure 21).

\footnotetext{
64 United States Congress, HR 1585-105 ${ }^{\text {th }}$ Congress, 22/7/1997.

65 Marc Bishon, "Record breaking stamp raises sticky questions", Cancerworld, 2006; November-December: 3235.

${ }^{66}$ Samantha King, Pink Ribbons Inc.: breast cancer and the politics of philanthropy, Minneapolis: University of Minnesota Press 2008:61.

${ }^{67}$ Editorial, How a US postal stamp helped fund a pivotal study on breast cancer, Associated Press, June 4, 2018.
} 

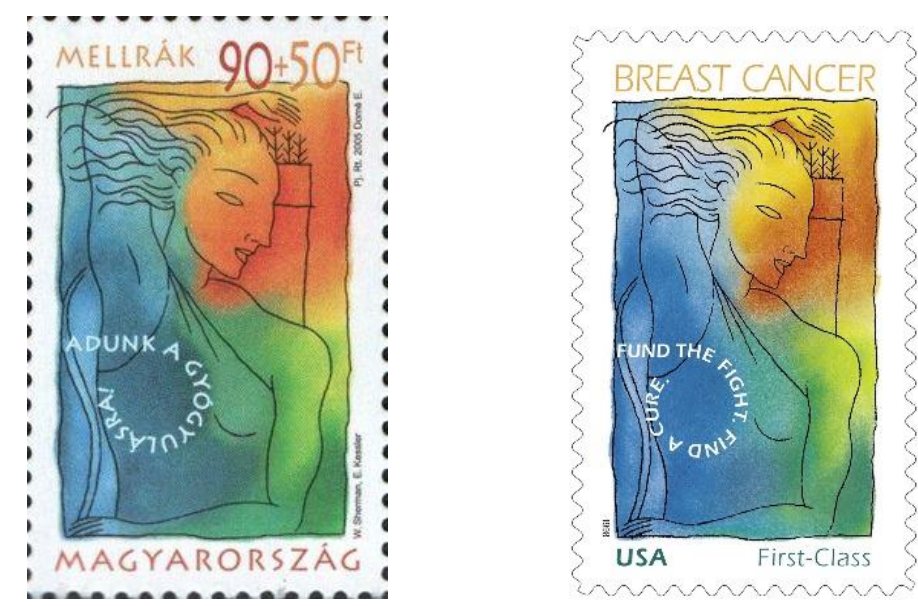

Figure 21. The Hungarian copy of the United States semi-postal stamp for breast cancer charities (2006) and the original United States stamp.

\section{Chapter 2.3 The Promotion of Health by Postage Stamps: A Background Review}

Over the last thirty years there has been a steady increase in the academic literature regarding the potential use of postage stamps in relation to health education and promotion. ${ }^{68}$ The early papers discussed the capability of mail services to deliver health messages via stamps, while others stressed the need for imaginative stamps to be used in this role. For many years, however, postage stamps had appeared from around the world featuring medical and health topics such as aspects of disease, distinguished doctors and nurses and the announcement of important health conferences. The majority of these issues could not be classified as health promotion although some may contain educational elements (see figure 22).

\footnotetext{
${ }^{68}$ O. A. Madsen, "Health messages on everybody's mail”, World Heath Forum, 1988; 9: 24-28.

M. K. Davies, "Health promotion with the help of the world's postal services", World Health Forum, 1989; 10 : 169-180.

M. K. Davies, "Health messages on postage stamps", World Health Forum, 1992; 13: 48-49.

E. Shafir, History and perspective of diabetes illustrated by postage stamps, London: Freund Publishing Ltd.,1999.

Ron Davis, Trish Groves, "Stamps for health education, British Medical Journal”, 2001;323: 969.

Robert A. Greenwald, "The postage stamp: carrying the mail and carrying the message", British Medical Journal, 2001; 323: 970.
} 

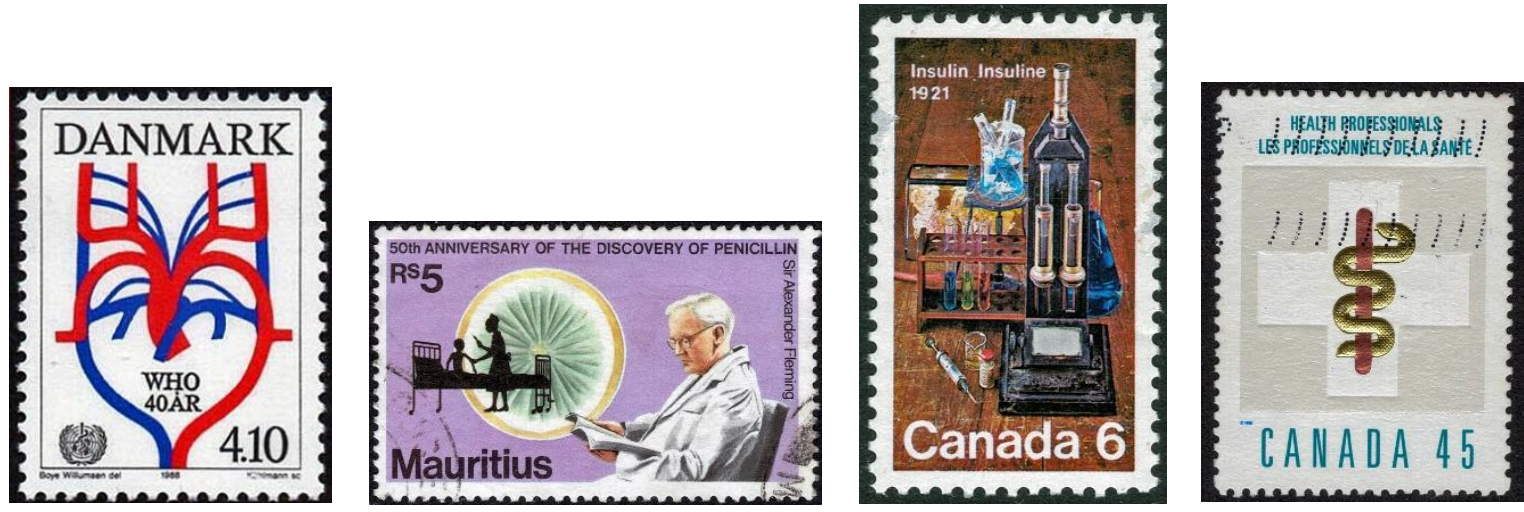

Figure 22. Examples of health related stamps that lack a direct health message. World Health Organisation, $40^{\text {th }}$ anniversary from Denmark, $50^{\text {th }}$ anniversary of Fleming's discovery of penicillin from Mauritius, the discovery of insulin in 1921, Canada, and a salute to the health professions, Canada.

This led, in due course, to many further publications regarding the relevance of stamps and health. ${ }^{69}$ A particularily relevant series was that published in the paediatric journal Archives of Disease in Childhood, that contained many stamps of an educational and health promotional nature (figure 23). ${ }^{70}$ Specific articles followed regarding individual country's issues of stamps in relation to health care, and prominent among those were Iran, Australia and Ceylon (Sri Lanka) (figure 24). ${ }^{71} \mathrm{~A}$ focus thereafter, was by papers reviewing the postage stamps of specific disease entities including cancer and genetics and medical procedures such as radiology (figure 24), and how stamps can be helpful in illustrating aspects of these diseases and diagnostic investigations. ${ }^{72}$

${ }^{69}$ Albert E. Chudley, J.C. Haworth, “Genetic landmarks through philately- haemophilia”, Clinical Genetics, 1999; 56: 279-281.

Manouri Senanayaki, "Paediatric Philately", Archives of Disease in Childhood, 1997; 76: 287-288.

70 M.K. Davies, A.J. Mayne, "Infant monitoring", Archives of Disease in Childhood, 2001; 84: 386.

71 John Pearn, "Further observation on the medical philately of Australia", Medical Journal of Australia, 1978; 2: 257-264.

Ahmadreza Afshar, "A brief Iranian medical history through commemorative postage stamps", Archives of Iranian Medicine, 2010; 13: 161-165.

Manouri Senanayake, "Health messages on postage stamps", Ceylon Medical Journal, 2003; 48: 56.

72 U. Sanyal, "Stamping out cancer", British Journal of Cancer, 2003; 89: 2165-2171.

Albert Chudley, 'Genetic landmarks through philately - a brief history of diabetes mellitus", Clinical Genetics, 1999; 55: 233.

Johannes Praestholm, "The origin and development of diagnostic radiology as illustrated by postage stamps", Acta Radiologica, 1997; 38: 930-936. 


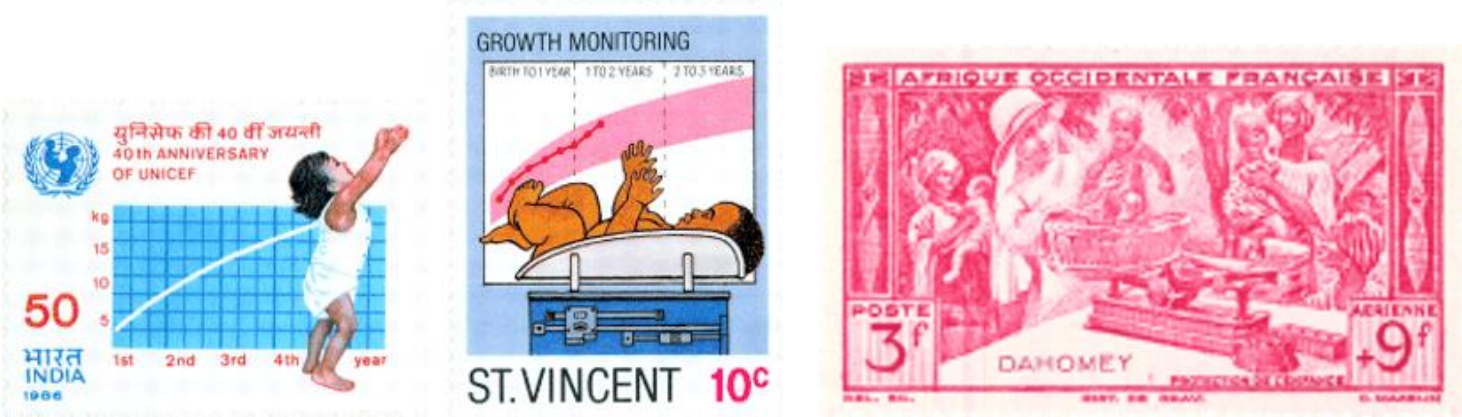

Figure 23. Infant growth monitoring, India (1986), St. Vincent (1987) and Dahomey (1942).
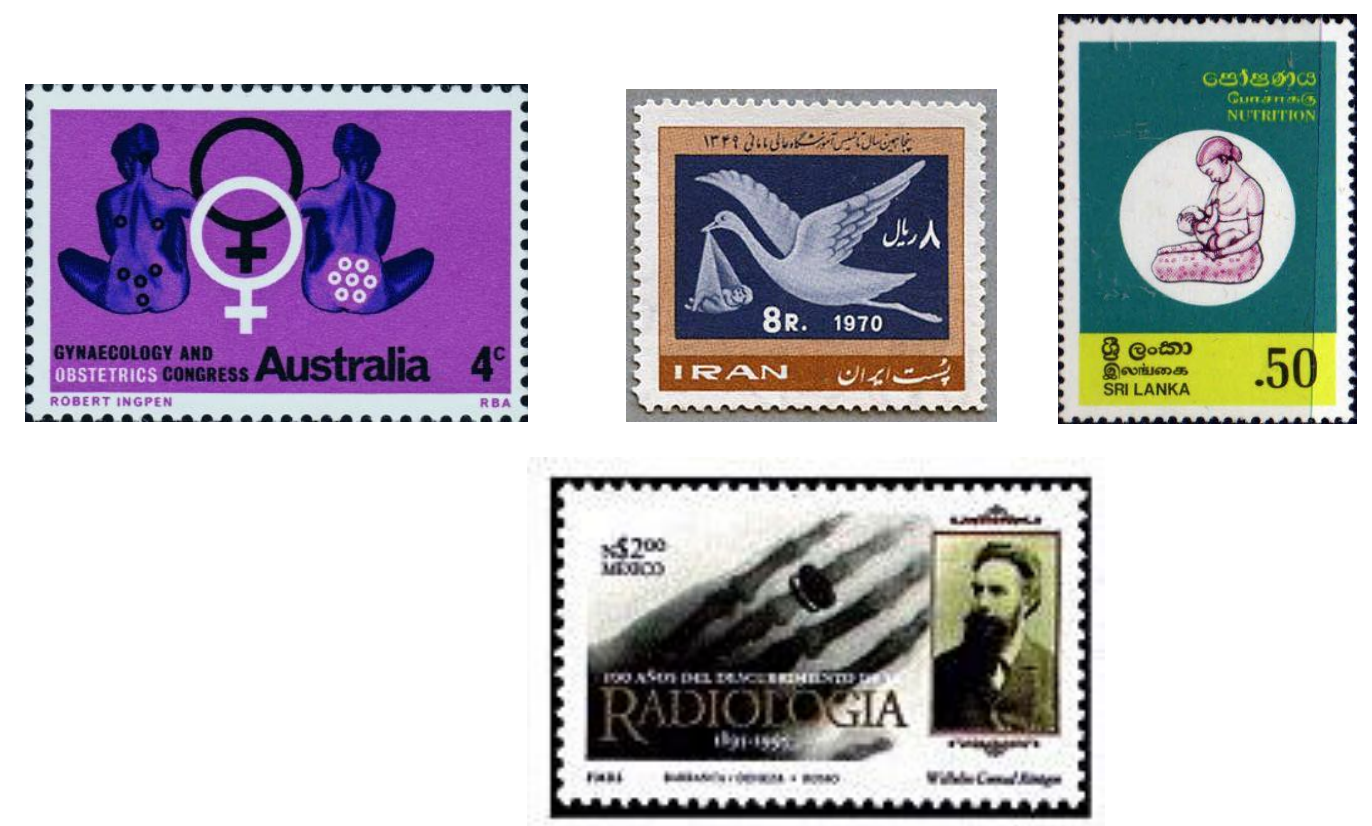

Figure 24. Australia, 1967, obstetric and gynaecology congress; Iran, 1970, Midwifery School; Sri Lanka, 1982, child nutrition policy; Radiology, Mexico, 1995, X-ray of Professor von Kolliker's hand , 1896, Röngen celebration.

Many nations have released postage stamps with a medical subject, health topic or medical anniversary theme. It was well into the $20^{\text {th }}$ century before interest was aroused by health professionals as to the potential educational value of these stamps. Apart from illustrating lectures and talks, it took a while before their potential and their wider use in health promotion was utilised. Again, it must be stressed, that semi-postal stamps raising funds for medical charities are not included in these observations nor are the perennial New Zealand "health stamps" that were overwhelmingly charity stamps. The recognition of the value of stamps in education was accompanied by the development of academic interest in the subject and subsequent pertinent publications. With the recognition of the capabilities of stamps, they became part of the educationalist's armamentarium to make the population aware of health issues and diseases and their need to seek help and treatment or alter their 
lifestyles. This approach will be discussed in the next chapter. Later the active adoption of health-oriented stamps that become part of an international health campaign will be developed later in this dissertation with the appropriate analysis of its value.

What stimulated the use of stamps in health education? In the $20^{\text {th }}$ century there were vastly improved printing and design capabilities and a rapid increase in postage stamp output of all types and themes. The emergence of new countries in the mid-1960s in Africa and Asia following decolonisation, resulted in the release of their own postage stamps, often in large numbers, as a form of national pride and to promote the recognition of their independence. In general, these countries were low income states, with poor health statistics and prone to tropical diseases and had high illiteracy rates. The visual image on the stamp provided an ideal medium to reach their populations with health information. Thus, from that period there was a marked increase in true health education stamps and a move away from the somewhat dull definitive stamps of the past towards message dominant comemmoratives. 


\section{Chapter 3. Health Education Through Awareness: What Constitutes a Health Educational or Promotional Postage Stamp.}

The World Health Organization has always used postage stamps as a means of information, of raising awareness, of prevention and of information. ${ }^{73}$

This chapter further explores the dividing line between health oriented stamps that may have been issued to promote a nation's medical and scientific achievements and /or issued merely to gain financial reward, and those stamps which are intended to have true educational or health promoting attributes.

With the increasing recognition that stamps can play an important role as messangers, many countries issued postage stamps to raise awareness locally and internationally about a variety of serious and important diseases and health issues. ${ }^{74}$ However, many postage stamps that appear to carry health related images were not issued for educational purposes. Among the reasons for their issue included the advertisement of forthcoming medical conferences, revenue for charities, national pride or simply to gain revenue for the postal authority. ${ }^{75}$

The purpose of this chapter is to determine if there are any obvious characteristics that make a stamp a true educational issue. A series of observational questions were devised, based upon the assumption that the stamp issue was released soley for the purpose of education in health and not for revenue purposes, advertisement of a medical or political event. The answers to these observations were scored as positive (1+) if indicating health education purposes and negative (1-) if an indication of a solely non-educational feature was made.

\footnotetext{
${ }^{73}$ Archives of the WHO: https://www.who.int/archives/fonds collections/special/philately/en/ accessed 3/12/2020

${ }^{74}$ Asiri Rodrigo, Jennifer Majoor, "Stamping the message: psychiatric themes in philately", Australasian Psychiatry, 2012; 20: 188-192.

Editorial, "United Nations postage stamps help fight on AIDS", AIDS Education and Prevention 1990; 2: 162163.

75 James Watson, “Thematic collecting', The Stanley Gibbons Book of Stamps, Ringwood: Stanley Gibbons Publications Ltd.,1990: 163.

James Mackay, "Kinds of stamps", The Guinness Book of Stamps, Enfield: Guinness Publishing Ltd., 1988: 134-175.
} 
The selection of the test subjects (stamps) was determined by seeking the first postage stamp identified as having a health-related topic in the Stanley Gibbons Stamp Catalogue for a specific country. ${ }^{76}$ A sample stamp was drawn, at random, from at least one country from each continent to give a wide base to the sample.

Table 1 lists the observational question posed and indicates the random "health" stamps selected for study. Table 2 lists the scores for each individual sample stamp and the overall scores for the 19 samples.

The question posed here, is as to the validity of many of these issues as being true health educational material or simply advertisements for conferences, for national pride, or simply to gain revenue for the postal agency or the national coffers from the philatelic world of dealers and collectors. To determine and address this dilemma, an unselected series of stamps that have been issued, to engender awareness of a range of medical conditions, and these are displayed in figure 25 . Based upon their images and text, this sample will be analysed to see what attributes they possess in terms of health education or promotion. A set number of devised criteria will be applied based upon the following questions and a score thus devised (see table 1 and table 2).

${ }^{76}$ Stanley Gibbons, Stamps of the World, London: Stanley Gibbons Ltd. 2018. 


\section{Table 1.}

Question, Score and Assessment Sheet

Yes/No

1. Does this stamp reflect a health or lifestyle topic?

$\mathrm{Y}=1$

2. Does this stamp carry a clear health message in its text?

$Y=1$

3. Does this stamp carry a clear health message in an image form? $\quad \mathrm{Y}=1$

4. Is there a text message other than one related to health? $\quad \mathrm{N}=1$

5. Is this a charity (semi-postal) fund raising issue? $\quad \mathrm{N}=1$

6. Is there an image related to a specific disease or life-style problem? $\quad Y=1$

7. Does it celebrate or advertise a health meeting, conference or anniversary? $\quad \mathrm{N}=1$

8. Does the stamp celebrate a country's health achievements? $\quad \mathrm{N}=1$

9. Does it celebrate an individual or groups of health professionals achievement? $\quad N=1$

10. Does this stamp have the appearance of being a commercial production with little regard for true health education or postage requirements? $Y=-1, N=1$

11. Overall can this stamp be regarded as having true educational value true? $Y=2$ 


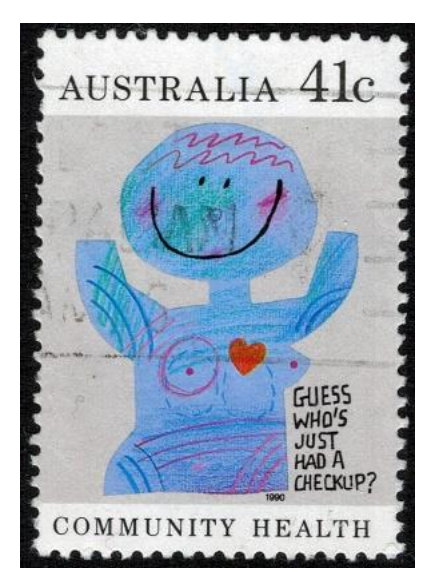

(A)

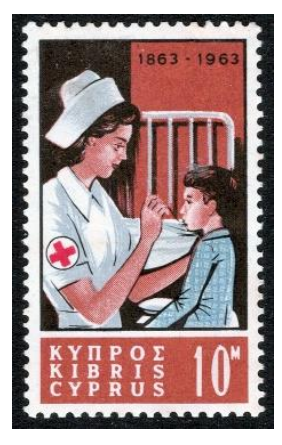

(D)

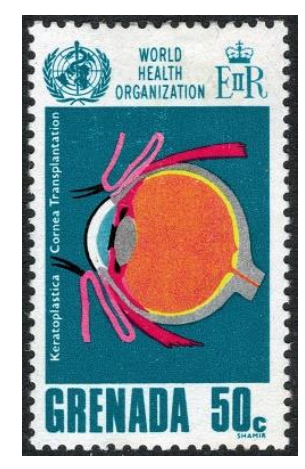

(G)

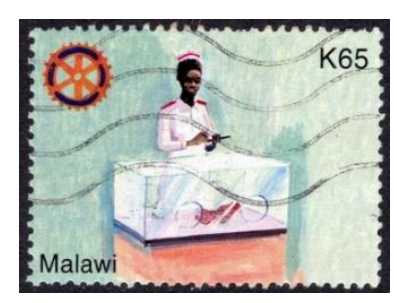

(J)

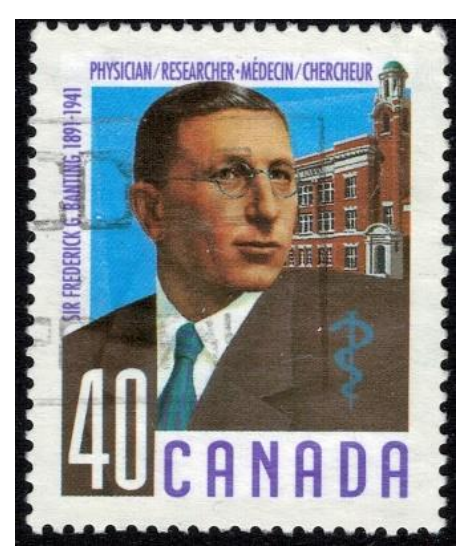

(B)

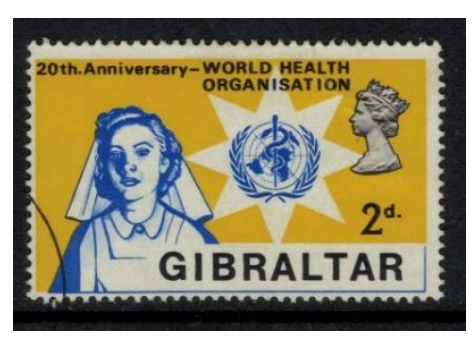

(E)

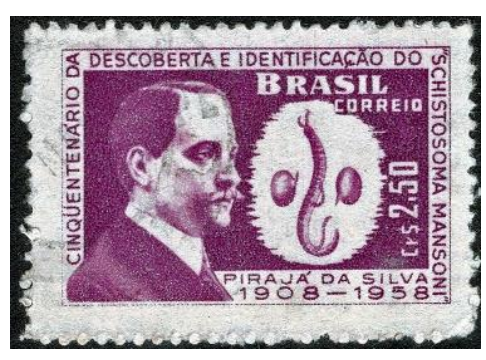

(H)

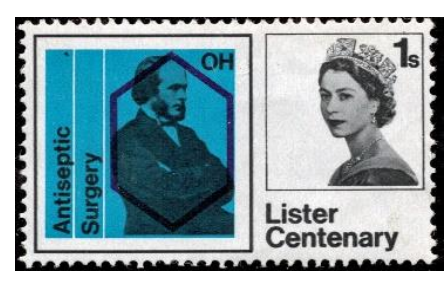

(K)

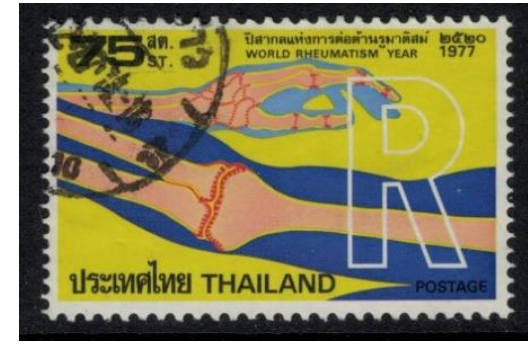

(C)

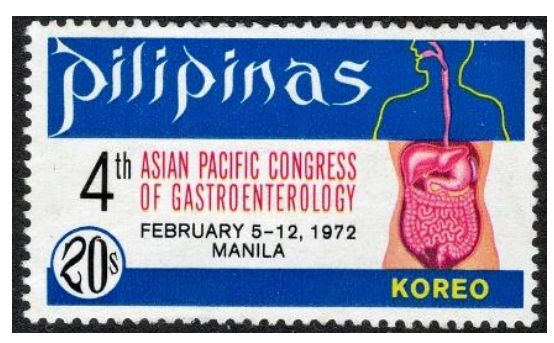

(F)

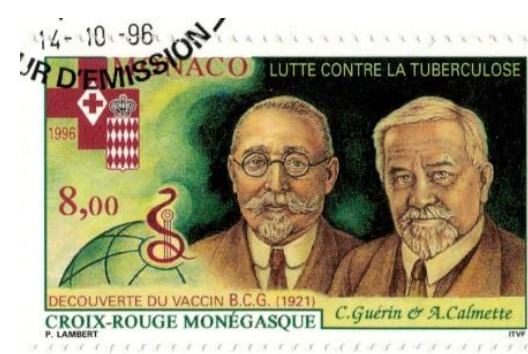

(I)

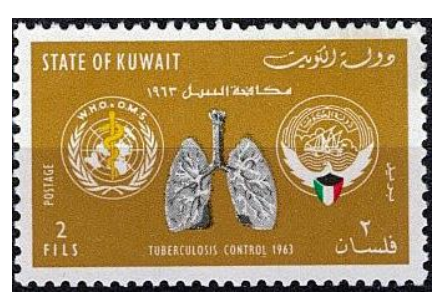

(L) 


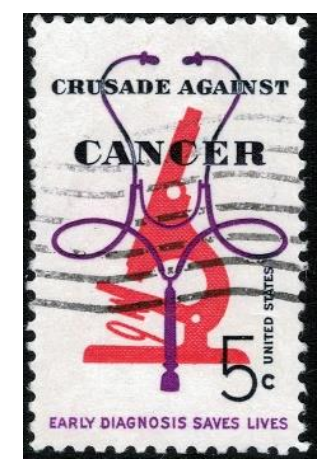

(M)

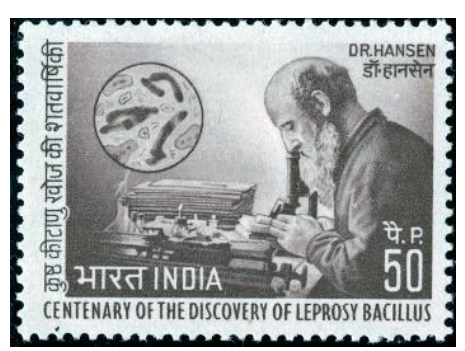

(P)

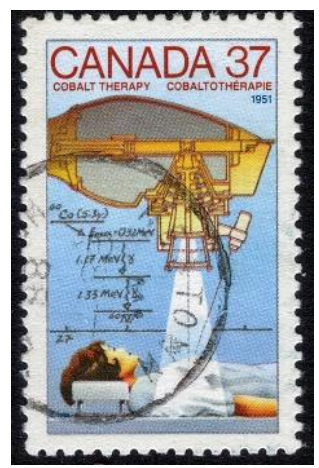

(N)

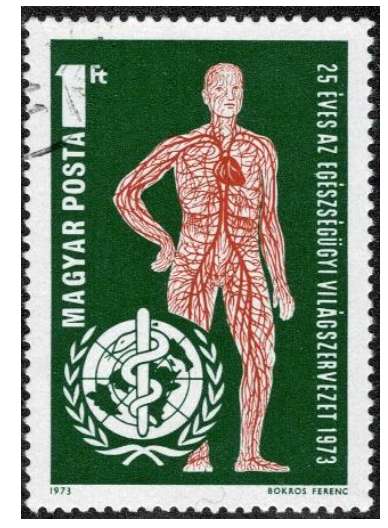

(Q)

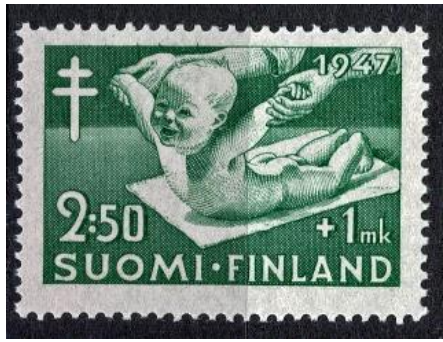

(0)

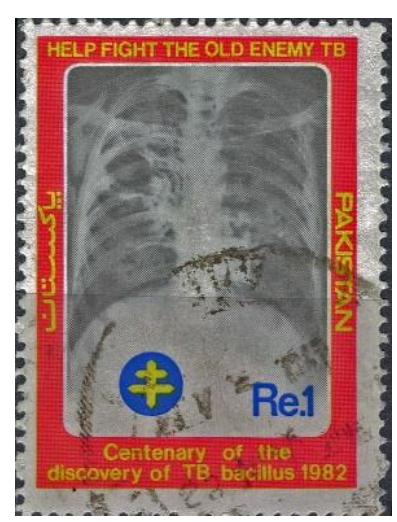

(R)

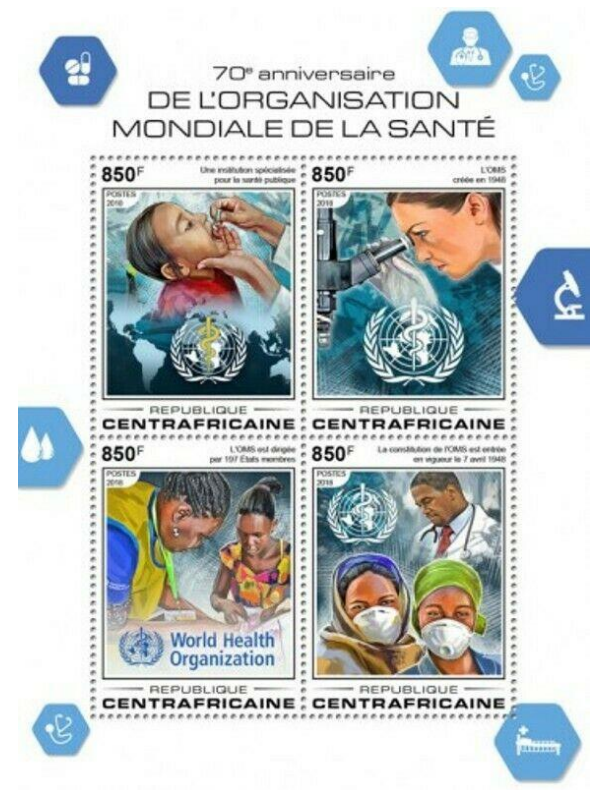

(S)

Figure 25. Nineteen health orientated stamps with varying attributes in terms of health education. 
Table 2.

\begin{tabular}{|l|l|l|l|l|l|l|l|l|l|l|l|l|l|l|l|l|l|l|l|}
\hline Stamp & A & B & C & D & E & F & G & H & I & J & K & L & M & N & O & P & Q & R & S \\
\hline $\begin{array}{l}\text { Question } \\
\text { Number }\end{array}$ & & & & & & & & & & & & & & & & & & & \\
\hline 1 & Y & Y & Y & Y & Y & Y & Y & Y & Y & Y & Y & Y & Y & Y & Y & Y & Y & Y & Y \\
\hline 2 & Y & N & N & N & N & N & Y & Y & Y & N & N & Y & Y & N & N & Y & N & Y & Y \\
\hline 3 & Y & N & Y & N & N & N & Y & Y & N & Y & N & Y & N & N & N & Y & Y & Y & N \\
\hline 4 & N & N & N & N & N & N & N & N & N & N & N & N & N & N & N & N & N & N & N \\
\hline 5 & N & N & N & N & N & N & N & N & N & N & N & N & N & N & Y & N & N & N & N \\
\hline 6 & N & N & Y & N & N & Y & Y & Y & N & Y & Y & Y & N & Y & N & Y & N & Y & N \\
\hline 7 & N & N & Y & Y & Y & Y & Y & N & N & N & Y & Y & N & N & N & Y & Y & N & Y \\
\hline 8 & N & Y & N & N & N & N & N & Y & N & N & Y & N & N & N & N & N & N & N & N \\
\hline 9 & N & Y & N & Y & Y & N & Y & Y & Y & Y & Y & N & N & N & N & Y & Y & N & Y \\
\hline 10 & N & N & N & N & N & N & N & N & Y & N & N & N & N & N & N & N & N & N & Y \\
\hline 11 & Y & N & N & N & N & N & N & N & N & N & N & N & N & N & N & N & N & Y & N \\
\hline Score & 11 & 5 & 8 & 5 & 5 & 7 & 8 & 8 & 6 & 8 & 5 & 9 & 8 & 8 & 6 & 8 & 6 & 12 & 5 \\
\hline
\end{tabular}

Key, $\mathrm{N}=$ No (does not apply), $\mathrm{Y}=$ Yes (applies). Potential total $=12$. 
From the above table it would appear that there may be a cut off level at a score of 9 , on the devised scale, that would discriminate between true health promotion/education stamps and other health related issues. Having applied the scoring method to a random number of 'health topic' stamps an attempt to validate this score was carried out. The scoring method was then applied to a series of specific health campaign stamps that had been previously issued for specific health education. These stamps are shown in the illustrated figures of Chapters 4-7 and the frontispiece. In addition two postage stamps selected from two prior dedicated health campaigns (not listed elsewhere), have been selected also in validation of the scoring method (figure 25). All stamps held true for a cut off scoring point of greater than 9 (of 12)

\section{A 1-2.}

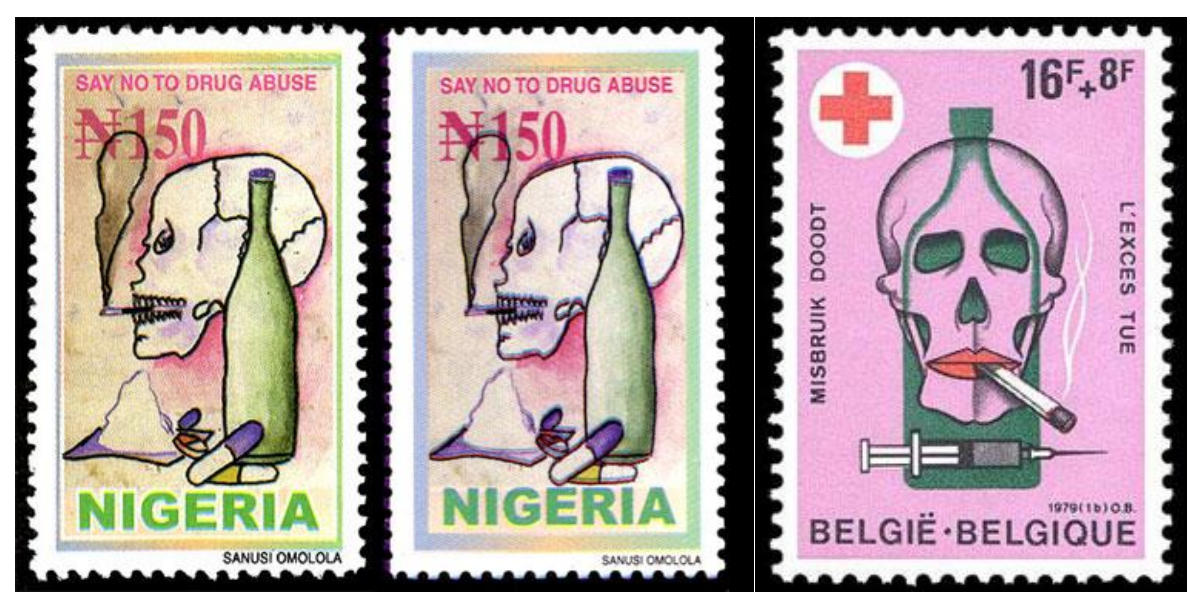

B 1-2.
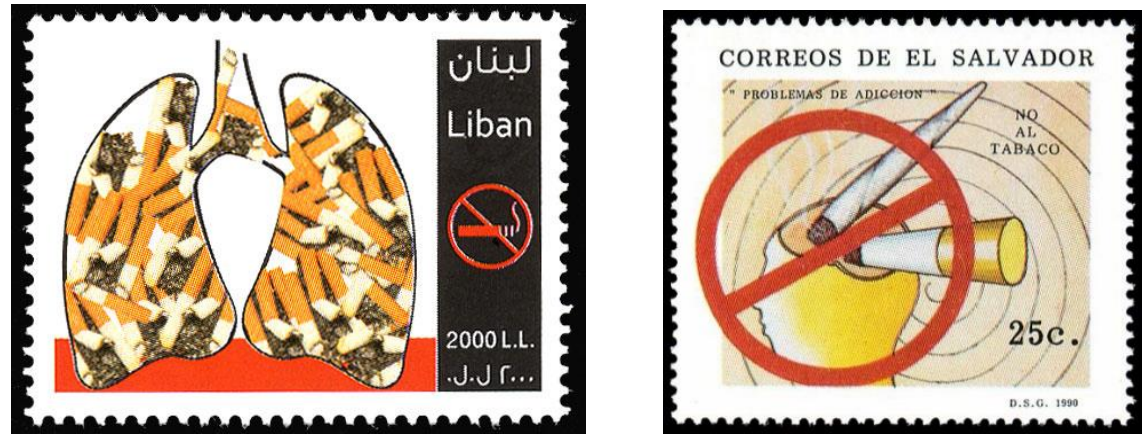

Figure 25.

Score: $A 1=12, A 2=12, B 1=11, B 2=12$ 
The selected health stamps clearly show that they have a score that is within the devised 'health stamp' range and this helps to validate the scoring method. In answering the dissertation's research question, this devised scale could prove to be helpful when determining whether a stamp is a true educational stamp or not, and assessing its contribution to health information and education. When applied to the New Zealand 'health' stamps the score offers confirmation that only two issues meet the criteria for true health promotion (see Chapter 2).

This chapter has been involved with determining an instrument that may be helpful in showing what attributes may be important in producing a successful health promotion stamp. Evans, in 1992, succinctly summed up what is being sought in these particular stamps when he stated "Stamps, which are the basis for circulation of correspondence, facilitate communication while simutaneously expressing certain ideas and emotions through their own imagery" ${ }^{77}$ It is these ideas that are required for education and not simply the reproduction of the face of a famous researcher or doctor. However, that is not to dismiss the inclusion of reference to the achievements by someone such as Robert Koch, in his discovery of tuberlce bacilli, when conveying information about tuberculosis. It is for these very reasons that this score was devised. Its purpose was to assist in differentiating true health promotion stamps from health topic stamps. This in turn was helpful aid when drawing conclusions to answer the posed research questions. This score, at best, can only be a rough guide as each stamp on a health topic may contain attributes that are not, at first sight, intended for an educational purpose.

There is one other aspect of health information and education by postage stamps, that may not fit exactly into the model described above. These stamps can be described as those raising public awareness and knowledge about social and health facts. A good example of this messenger is the stamp that was issued by the United Nations in 2020 to celebrate the $40^{\text {th }}$ anniversary of the eradication of small pox (figure 26 ).

\footnotetext{
77 K. Evans, "The argument of images: historical representation in solidarity, underground postage 1981-87", American Ethnologist, 1992; 19: 749-767.
} 


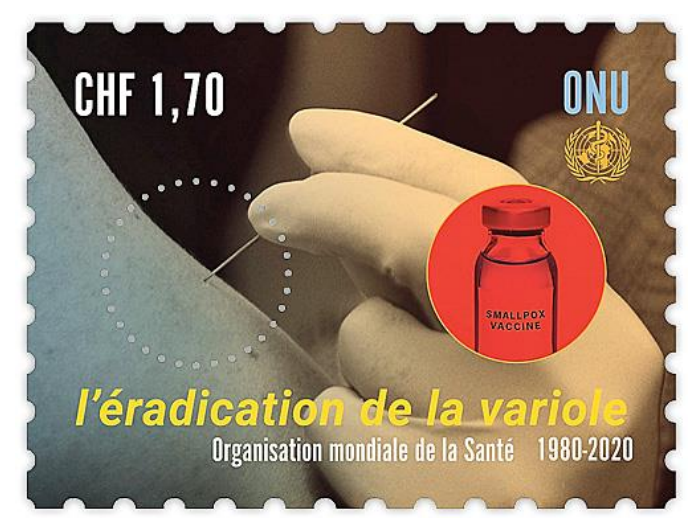

Figure 26. United Nations and World Heath Organisation stamp of 2020, $40^{\text {th }}$ anniversary of the Score by small pox vaccination.

For 3,000 years small pox was a scurge of mankind killing millions of people. Its elimination was a success that can be attributed to immunisation and global solidarity. In relation to health promotion, the United Nations had this to say:

"The successful smallpox eradication programme yielded vital knowledge and tools for the field of disease surveillance, the benefits of ring vaccination and the importance of health promotion in fighting diseases such as poliomyelitis and the Ebola virus. It also laid the foundation for stronger national immunization programmes worldwide, underpinning the establishment of primary health care in many countries and creating momentum toward Universal Health Coverage" ${ }^{78}$

It could be argued that this stamp is a useful messenger as it supports the success that can be achieved by vaccination and serves as a reminder that at the times of the Covid 19 pandemic what is possible by nations working together. The World Health Organization stated:

“When the WHO's small pox eradication campaign was launched in 1967, one of the ways countries raised awareness about smallpox was through postage stamps". ${ }^{79}$

\footnotetext{
78 UN smallpox stamp: https://www.un.int/news/un-stamp-commemorates-40th-year-smallpox-eradication accessed 6/6/2020.

79 Smallpox campaign: https://news.un.org/en/story/2020/05/1063582 accessed 6/12/2020
} 
Hence, the use of postage stamps could be instrumental in encouraging immunisation for other conditions and also support the international moves to eliminate poliomyelitis from the world in a similar fashion to that of small pox.

During the period, 1925-1965, in an attempt to raise social awarenes of tuberculosis and raise funds, Belgium issued 39 postage stamps, all semi-postal charity stamps. The only indication that the surcharge went to tuberculosis charities was the addition of the tuberculosis logo, the Cross of Lorraine. There were a number of different designs on these stamps over the period, but none carried a text or image giving health information about the disease. The exception was a single issue showing a sanatorium. In terms of the thrust of this dissertation, the production of these stamps, like the New Zealand health stamps, represents a lost opportunity. While charity stamps per se fulfil a function of raising money for health projects or research, they are, themselves instruments of potential health information or behavioural change. They do not qualify as miniature health messengers nor do they educate the population about this killer disease, its prevention or treatment. Other countries performed no better. Finland issued 27 tuberculosis charity stamps between 1946-1983, and again their sole gesture to education was the display of a modern sanatorium (see figure 27 ).

A final example of social awareness by postage stamps are those stamps issued that have attempted to raise the profile of diseases and disorders of vision. There have been so many of these that they have formed the basis of a published article on eye diseases and stamps. ${ }^{80}$ Figure 28 shows examples of four of these stamps that just fail to meet the criteria of health education or promotion but have attributes that may raise the population's awareness of matters relating to the eye and its disorders.

${ }^{80}$ Thierry Zeyen, The story of ophthalmology told by postage stamps, Clermont Ferrand: Laboratoires Thea, 2019. 

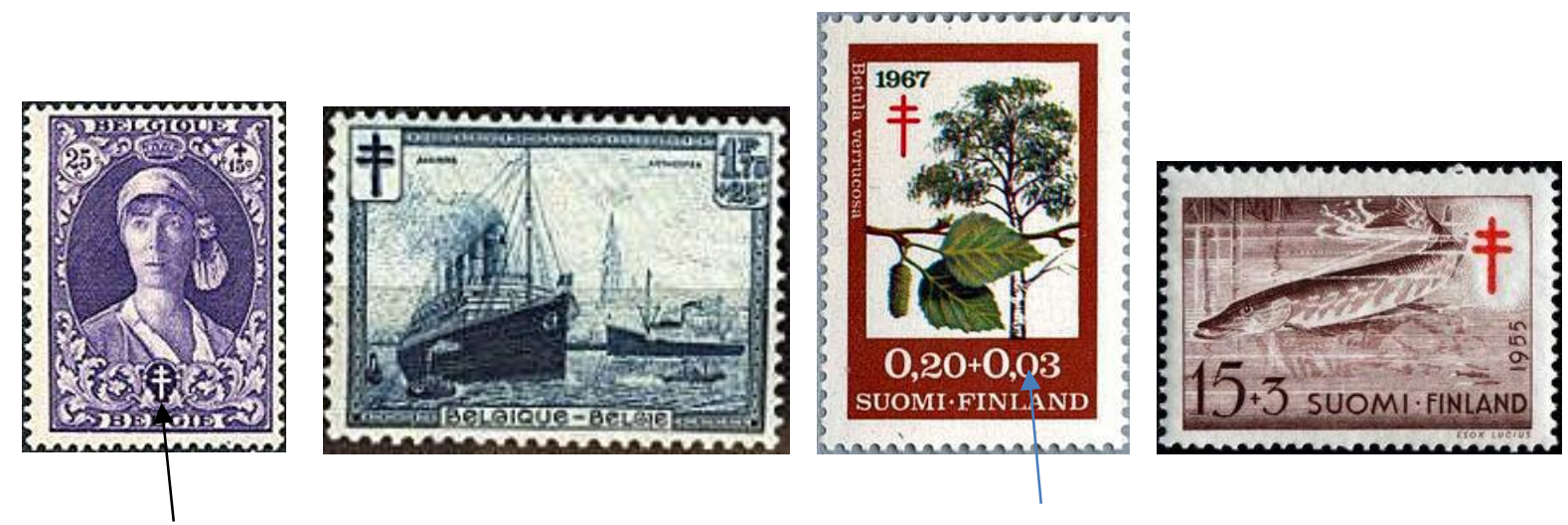

Figure 27. The only indication that these stamps carry a surcharge for the Tuberculosis Fund, is the Cross of Lorraine-black arrow and the surcharge value-blue arrow. Belgium: Queen Elisabeth, 1931, ships, 1929, Finland: birch tree, 1967, fish, 1955.
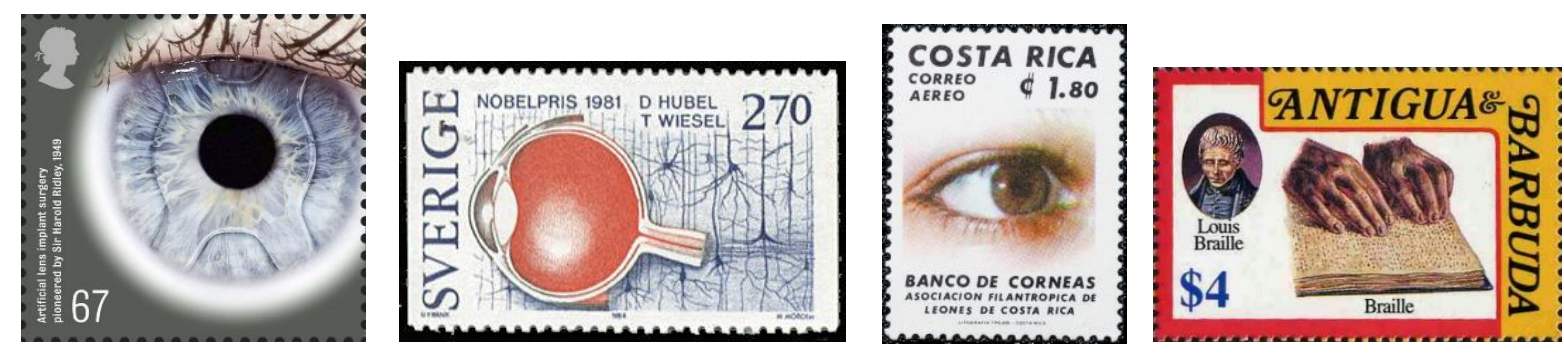

Figure 28. Examples of eye-related themes of some educational value: United Kingdom, artificial lens, 2018, Sweden, eye anatomy, 1984, Costa Rica, cornea banks, 1981, Antigua and Barbuda, braille, 1992.

This chapter has focussed upon what constitutes the basis of a health promotion or education stamp. The image and its design are of paramount importance and the accuracy of any text is vital, the latter having been stressed here with the example of the American breast cancer issue. The text itself, however, is not essential for the stamp to have the necessary impact. Conversely, as shown in the Indian printed postal material, an effective health message can be relayed even in the presence of a mundane stamp or a printed likeness.

By necessity, the analysis has been ongoing throughout this chapter because of the establishment of the scoring system. The purpose of this was to create a yardstick by which to judge the myriads of stamps with a health or medical theme, be they fund raising or commemorative, and determine if they had true health educational value. It would appear that a score of 9 or above indicates that the stamp may be classified as an health education message, although further validation will be required. 
There seems to be a borderland in which some stamps may have a health awareness content but were not designed specifically to be educational and by happenstance have these attributes. The ophthalmology selection fits into this category and the small pox anniversary stamp and similar issues raises other more subtle avenues for health promotion.

At this point, the characteristics of a desired educational stamp have been described and examples demonstrated. The next chapter looks at a health campaign during which stamps have been specifically designed to complement and to convey relevant health information to be issued in parallel with the running of the active therapeutic and educational programme. 


\section{Chapter 4. Heath Campaigns: an Important Role for the Postage Stamp as a Health Messenger in Malaria}

'The Campaign against Malaria' (Lutte contra le Paludisme) was a global health initiative launched by the World Health Organisation (WHO) in 1955 with the goal of eliminating malaria world-wide within five years. The plan was prefaced on the fact that there had been success already against the anopheles mosquito with the use of DDT. ${ }^{81}$ The international campaign was to be funded by the member states of the WHO and was to be the most grandiose project attempted by the WHO since its inauguration. Five years prior to the start of the campaign, in May 1950, the Indian delegation at the WHO proposed that special postage stamps, be prepared and issued, to help promote international health by the WHO and perhaps fund its activities. ${ }^{82}$

This idea took hold, and the concept of special stamps led to the proposal being formally accepted by the organisation. The Director-General of the WHO expressed his satisfaction with the plan to "issue postage stamps to increase public information on and stimulation of interest in malaria eradication". ${ }^{83}$ Matters progressed quickly, thereafter, and the following statement was released by the WHO as the first stamp from Taiwan was welcomed (figure 29):

At its 26th meeting the Executive Council of the World Health Organization (WHO) adopted a resolution, dated the $28^{\text {th }}$, October 1960, requesting all its member countries to issue simultaneously on April 7, 1962 a set of Malaria Eradication Postage Stamps, with the further support and assistance of the Universal Postal Union (UPU). The main purpose of the program is to arouse public interest in antimalaria work, thus facilitating the accomplishment of the worldwide malaria eradication scheme. The anti-malaria drive launched in Taiwan in 1952 with the

\footnotetext{
${ }^{81}$ DDT- Dichlorodiphenyltrichloroethane, an organochloride insecticide first synthesised in 1874 and generally banned for agriculture by 1972.

82 Indian proposal:

https://apps.who.int/iris/bitstream/handle/10665/100014/WHA3 91 eng.pdf?sequence=1\&isAllow ed=y accessed 10/6/20.

${ }^{83}$ Director-general of WHO statement:

https://apps.who.int/iris/bitstream/handle/10665/88730/EB34R18 eng.pdf?sequence=1\&isAllowed=y accessed 10/6/20.
} 
cooperation of WHO made such headway in the following ten years that malaria was almost wiped out. With a view to intensifying the anti-malaria campaign and responding to an international move of such significance, a set of "Malaria Eradication" commemorative stamps was issued in honour of a joint suggestion made by both the WHO and UPU. The stamp has as its central design the globe emblem of WHO above an anopheline mosquito, symbolic of the world-wide drive against malaria. ${ }^{84}$

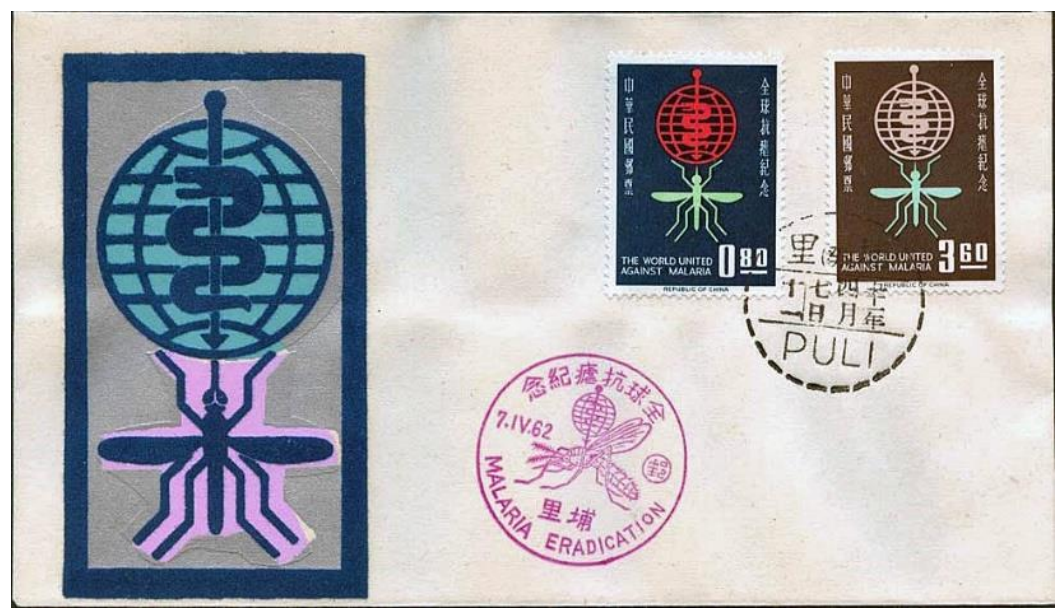

Figure 29. First day cover of the new malaria stamps from Taiwan, 7 April 1962, 2.6 million of these stamps were issued.

A semi-standard format was adopted by many countries which followed that shown in figure 29 and included the same symbols. The globe represented the world and the WHO, while Aesculapius's snake symbol represented the medical attack on the mosquito that, in turn, represented malaria. Between 1962-1963, 140 countries took up the challenge and issued postage stamps or appropriate postage cancellation slogans. However, not all countries adhered to the standard format as that shown in figure 29. While the standard stamp did raise awareness to the anti-malaria campaign, it was not very helpful in revealing the measures necessary to eliminate the disease. Fortunately, many countries felt more specific designs were indicated and released stamps with more appropriate images. Most of these stamps addressed the health promotion aspects needed to complement the actual

\footnotetext{
${ }^{84}$ Stamp releases: https://www.post.gov.tw/post/internet/W stamphouse/index en.jsp?ID=2807\&file name=B077 accessed 10/6/20.
} 
malarial eradication programme. None of the issued stamps were semi-postal charity stamps as had been proposed initially but many countries donated over 100,000 stamps to the United Nations to raise funds for the campaign.

The global campaign's stated strategy emphasised the importance of vector elimination and further control with DDT spraying, adherence to treatment protocols and regimens as well as ongoing close surveillance of the programme itself. Thus, breaking the cycle of breeding and subsequent infection was a paramount theme. It was these major themes at which stamp design was targeted when the issuing authority choose formats other than the basic design.

A selection of issued stamps which meet the criteria of health promotion are presented for each of the primary malarial strategies. This will allow evaluation as to how these stamps further the educational aspects of the campaign and if they actually met those aims. In addition, a brief background to the reasons for the various strategies used in the eradication of the disease will be added.

\section{Interference with the breeding cycle}

The Anopheles mosquito is the primary vector of malaria and the female deposits up to 200 eggs at one session on the surface of water. The eggs remain on the surface by a float mechanism and hatching occurs between two days and three weeks depending upon the ambient temperature. During her short life span of a few weeks the female can lay many thousands of eggs. The site of egg deposition is fresh or saltwater in ground pools, irrigated land, marshes or places with slow moving water. ${ }^{85}$ The stages of development are shown in figure 30 and the Nigerian stamp portraying the same event. Several of the issued stamps depict the environment in which the mosquito lays her eggs and draw attention to the marshy ground that is a very typical location (figure 31). It is therefore this type of water site that active prevention attacks were to be directed to disrupt breeding and hence break the reproductive cycle.

\footnotetext{
${ }^{85}$ Hamza AM, El Rayah EL., "A Qualitative Evidence of the Breeding Sites of Anopheles arabiensis Patton (Diptera: Culicidae) in and Around Kassala Town, Eastern Sudan", International Journal of Insect Science, 2016; 8: 65-70.
} 

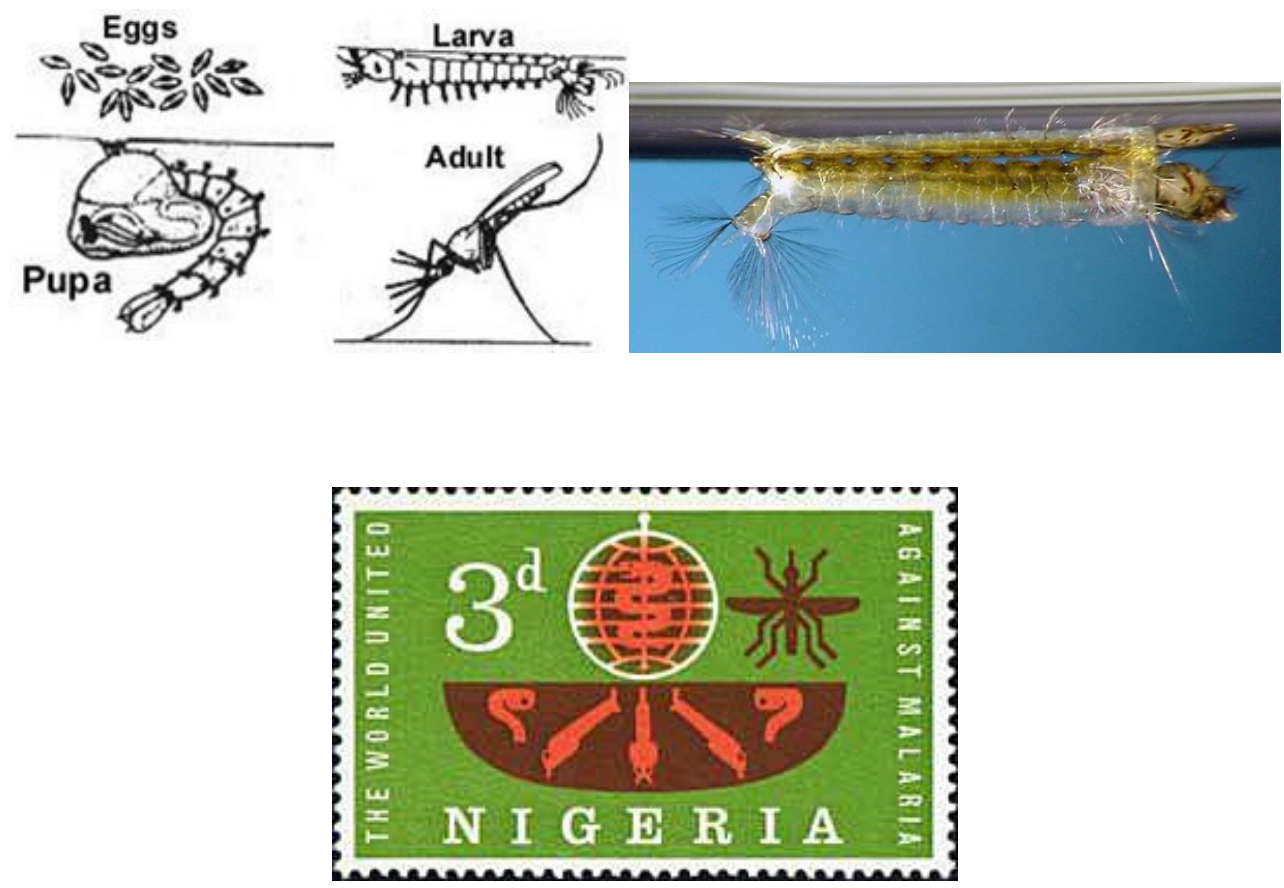

Figure 30. The diagram shows the various stages of the mosquitoes' development in water from egg to adult insect, and the photograph is of the larval stage, while the stamp illustrates whole process. Nigeria 7, April 1962. Photo source: New South Wales Health, S. Doggart.
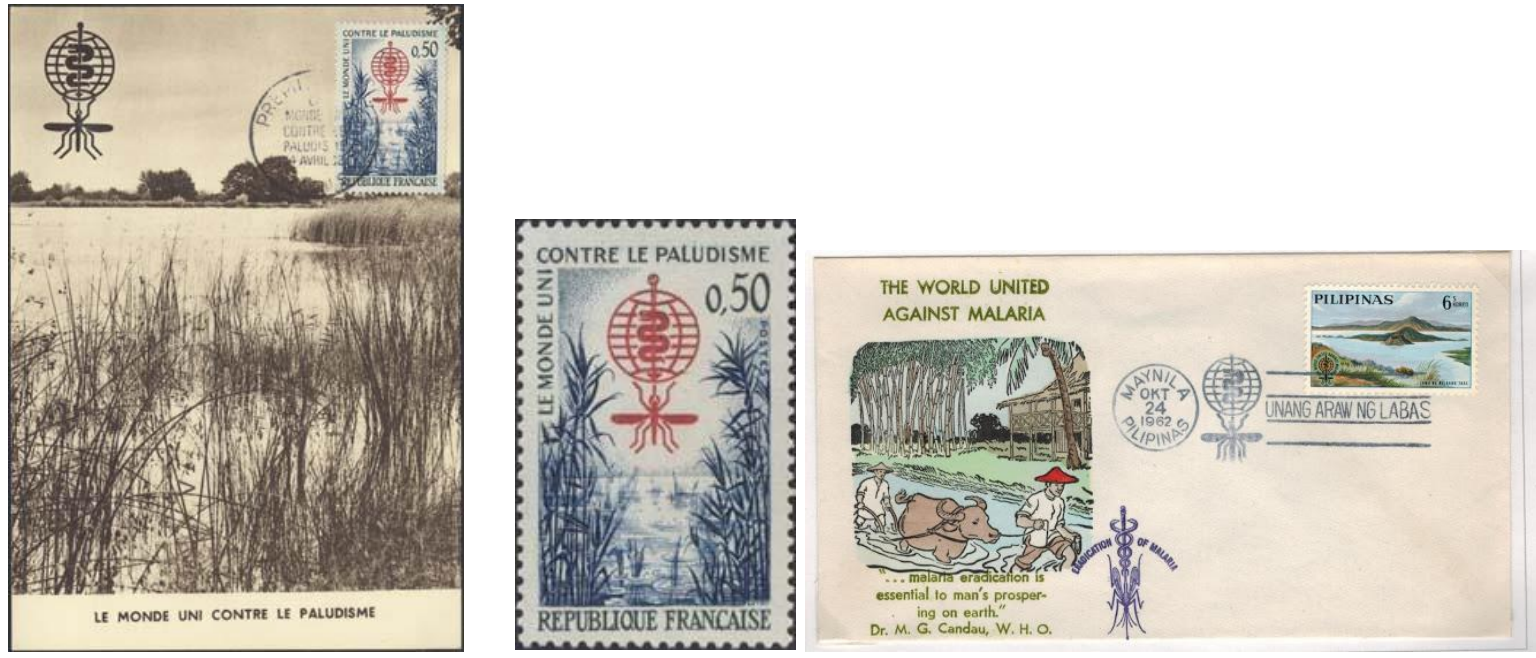

Malarial breeding environment, France postal maxicard and malaria campaign stamp, Philippines First Day cover of possible malarial sites in that country, 1962. 

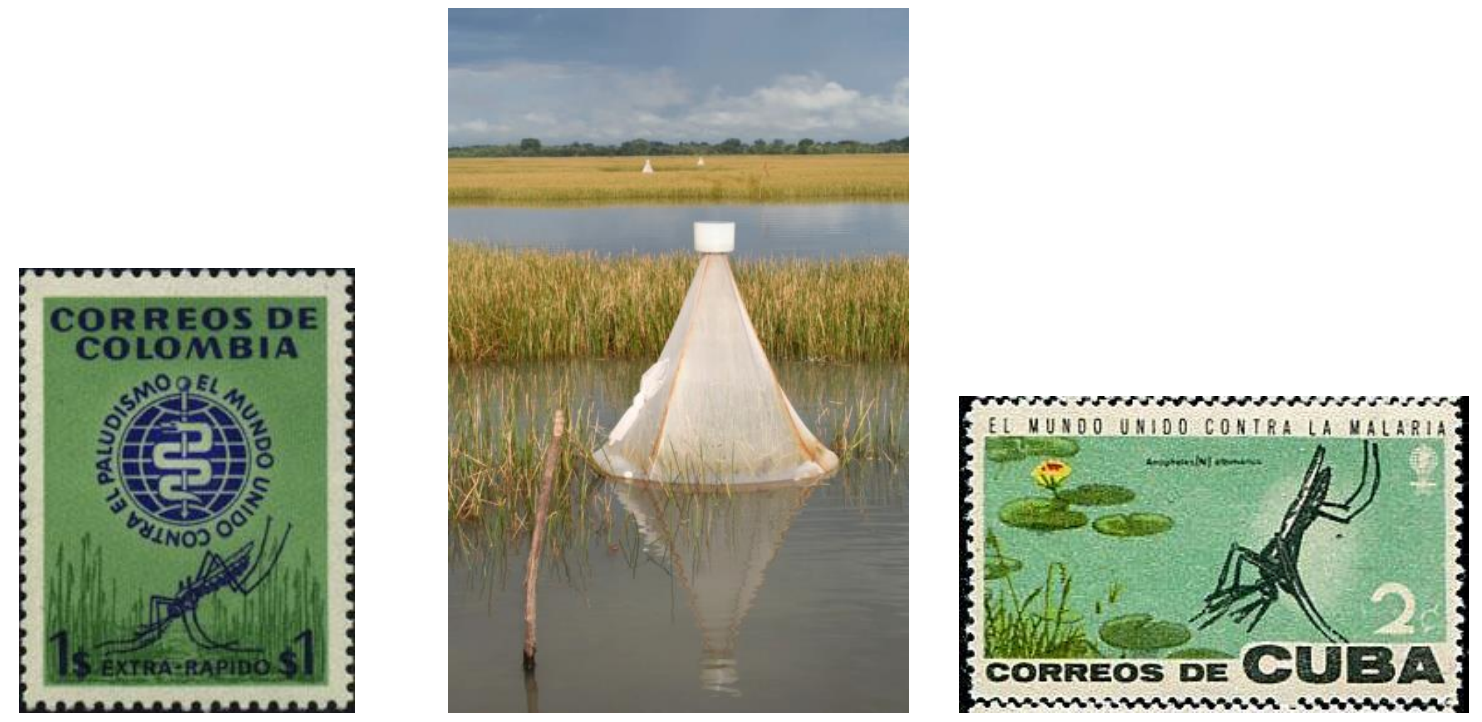

Figure 31. Malarial breeding areas, Colombia and Cuba, Photograph: estimating the vector production by habitat. ${ }^{86}$

\section{Identifying and eliminating the adult mosquito}

Many contributing countries used large images of the adult mosquito and diagrammatic representations of insect killing on their malaria stamps presumably to educate and encourage their people to identify and eliminate any mosquito they encountered by physically destroying them. Examples of these types of stamp are reproduced in figure 32 .

\section{DDT spraying of swamps and houses}

At the onset of the "World against malaria" campaign, it was considered that the liberal use of DDT could be a major factor in the plans for the elimination of malaria from the world. Prior to the campaign, DDT was shown to be a potent insecticide, first in South Africa, then in India where a marked decrease in malaria occurred after widespread spraying with the agent. In Ceylon (Sri Lanka) following DDT usage the malaria case rate, in 1964, fell from the prior 2.8 million per year to 110 . It was soon recognised that indiscriminate spraying of DDT was upsetting the balance of nature when $90 \%$ of insects regarded as good were being killed. Further repercussions occurred upon the ecosystem with the deaths of fish and birds

\footnotetext{
${ }^{86}$ Ulrike Fillinger, Heleen Sombroek, Silas Majambere, et al., "Identifying the most productive breeding sites for malaria mosquitoes in The Gambia", Malaria Journal, 2009; 8: 62.
} 
and by 1972 , controls on DDT use were introduced. By this time 19 species of mosquito had developed resistance to its effects and DDT had entered into the food chain. ${ }^{87}$ The above facts need to be born in mind, when examining the host of stamps that were issued supporting and encouraging widespread spraying with DDT. At this time the dangers were not appreciated. Figure 33 gives examples of the "health" promotion against malaria by encouraging DDT spraying.
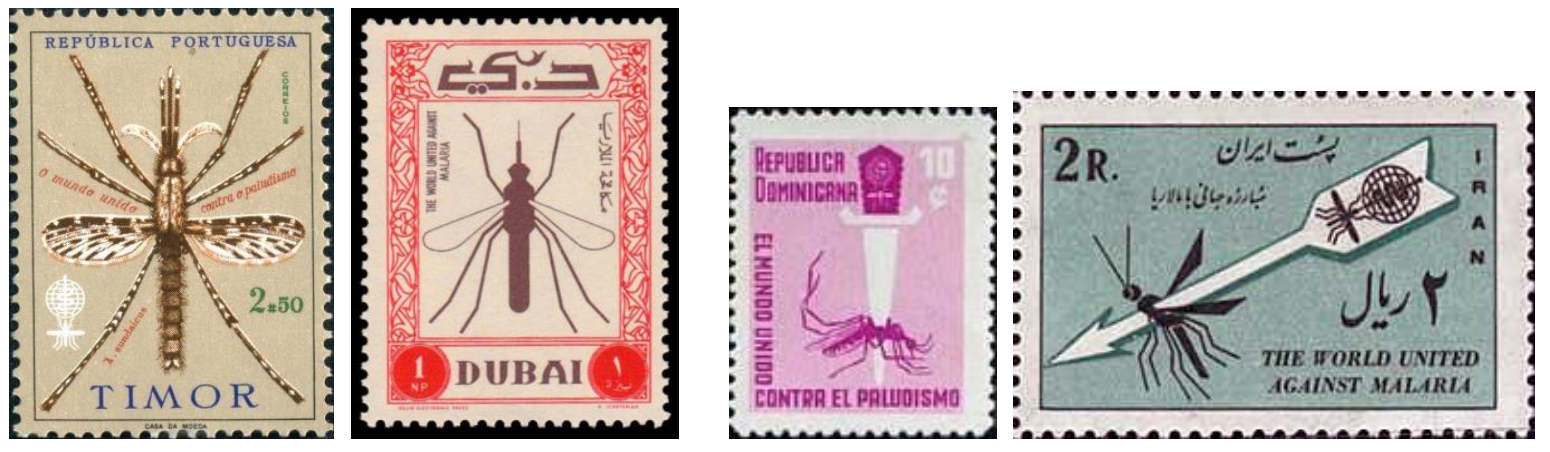

Figure 32. Anopheles mosquito portrayed by Timor and Dubai. Encouragement to elimnate the mosquito, Dominican Republic, Iran, 1962.
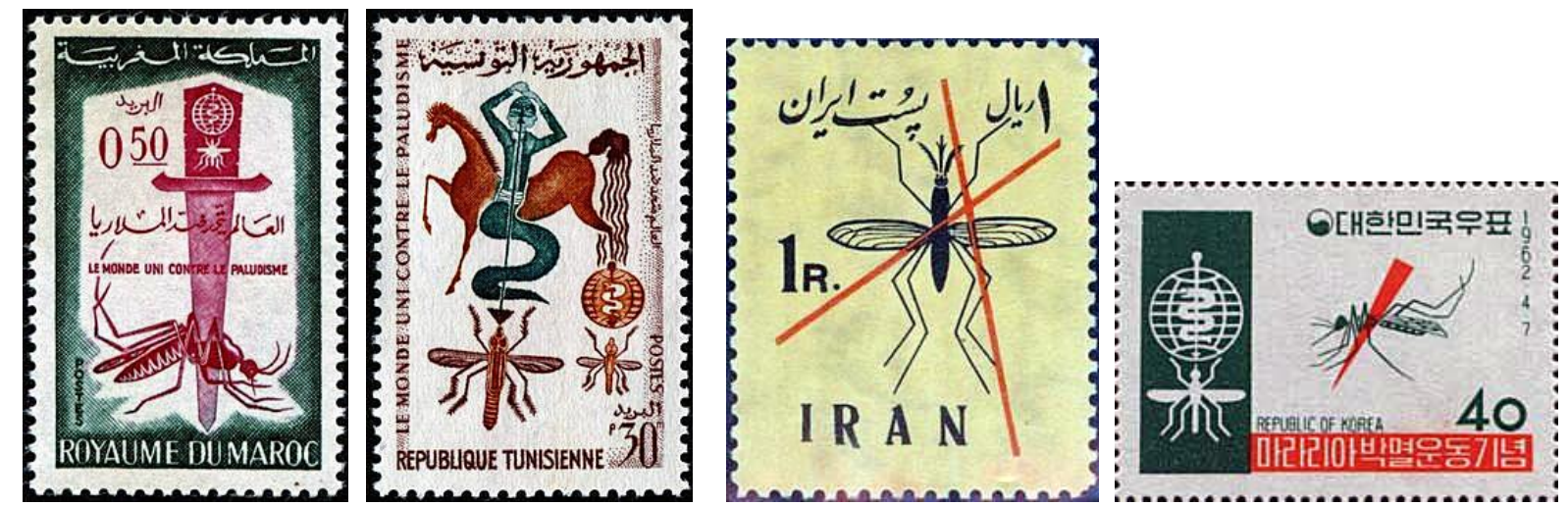

Further examples of mosquito elimination, Morocco, Tunisia, Iran and South Korea, 1962.

\footnotetext{
${ }^{87}$ Christobal S. Berry-Cabal, "DDT and silent spring: fifty years after", Journal of Military and Veterans Health, 2011; 19: 19-24.
} 

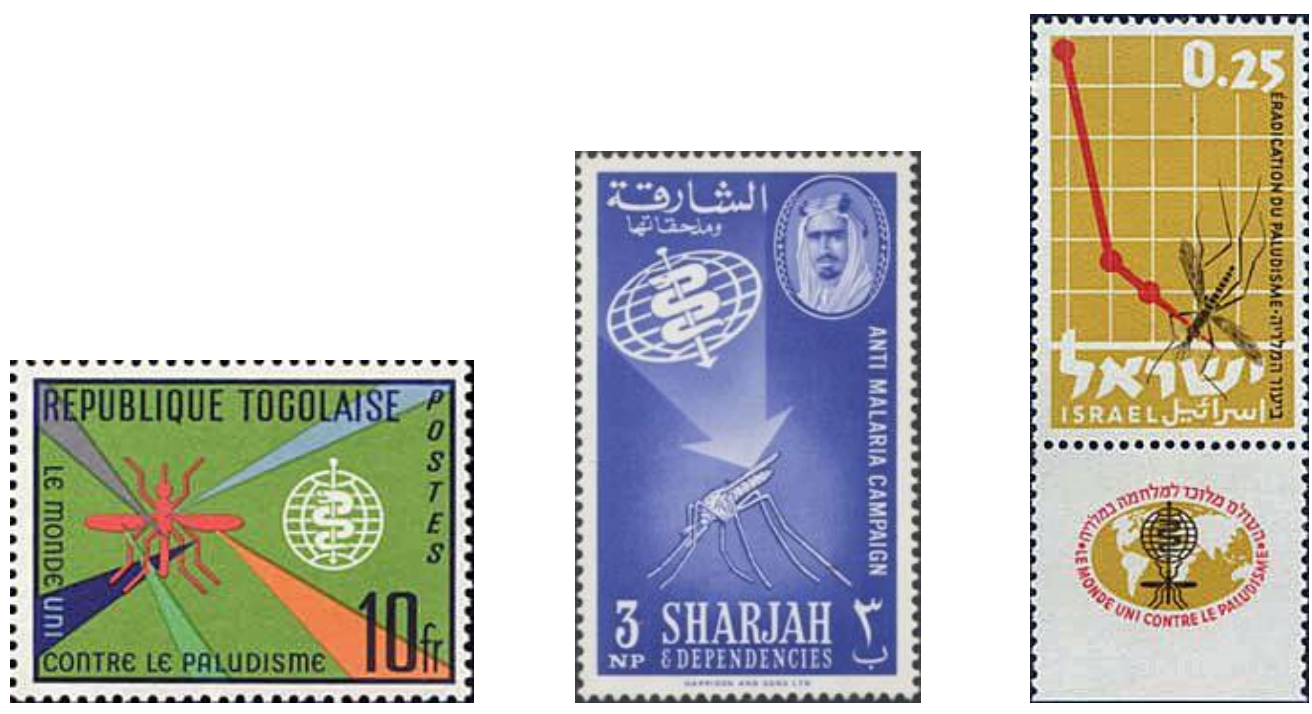

These stamps indicate the wide range of countries advocating anopheles insect destruction; Togo, Sharjah and Israel.
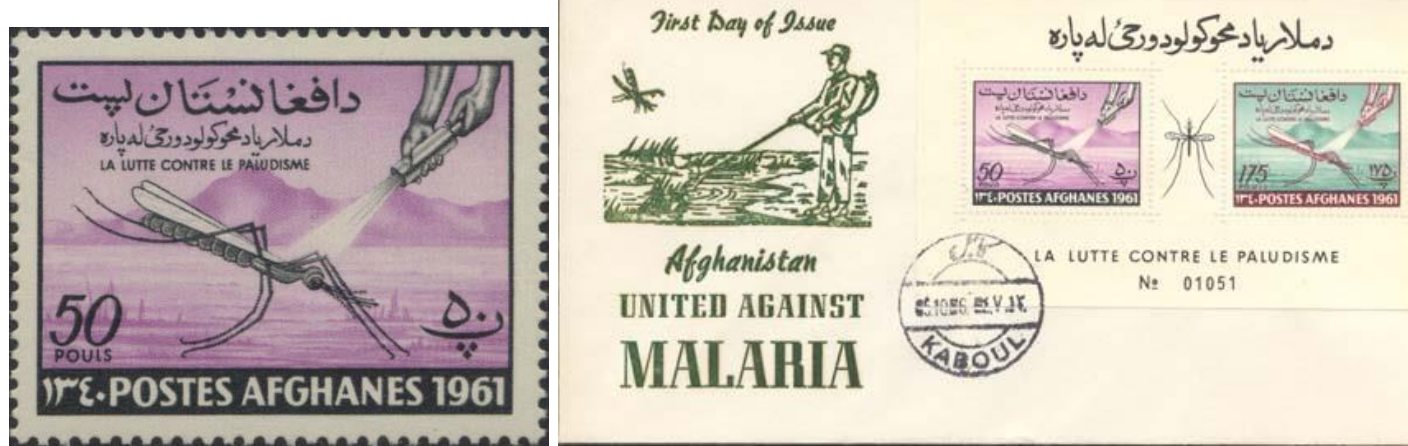

Afghanistan

UNITED ABAINST

МАІАНІА

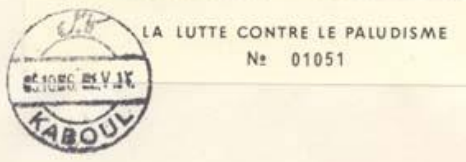

Active spraying in wet areas against malarial carrying insects, Afghanistan with First Day Cover, 1962.
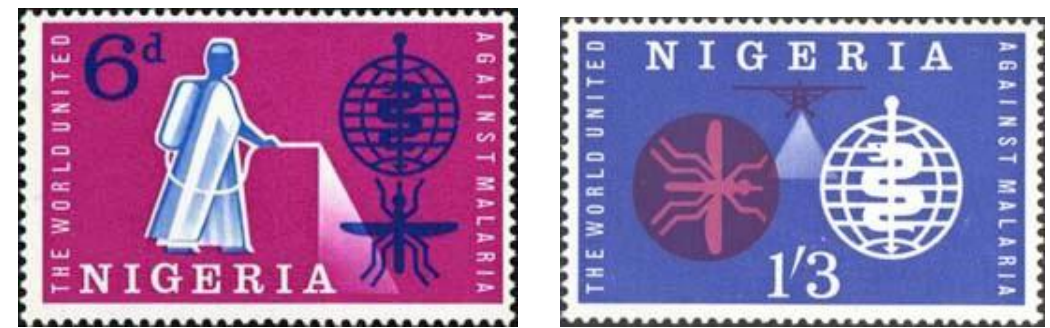

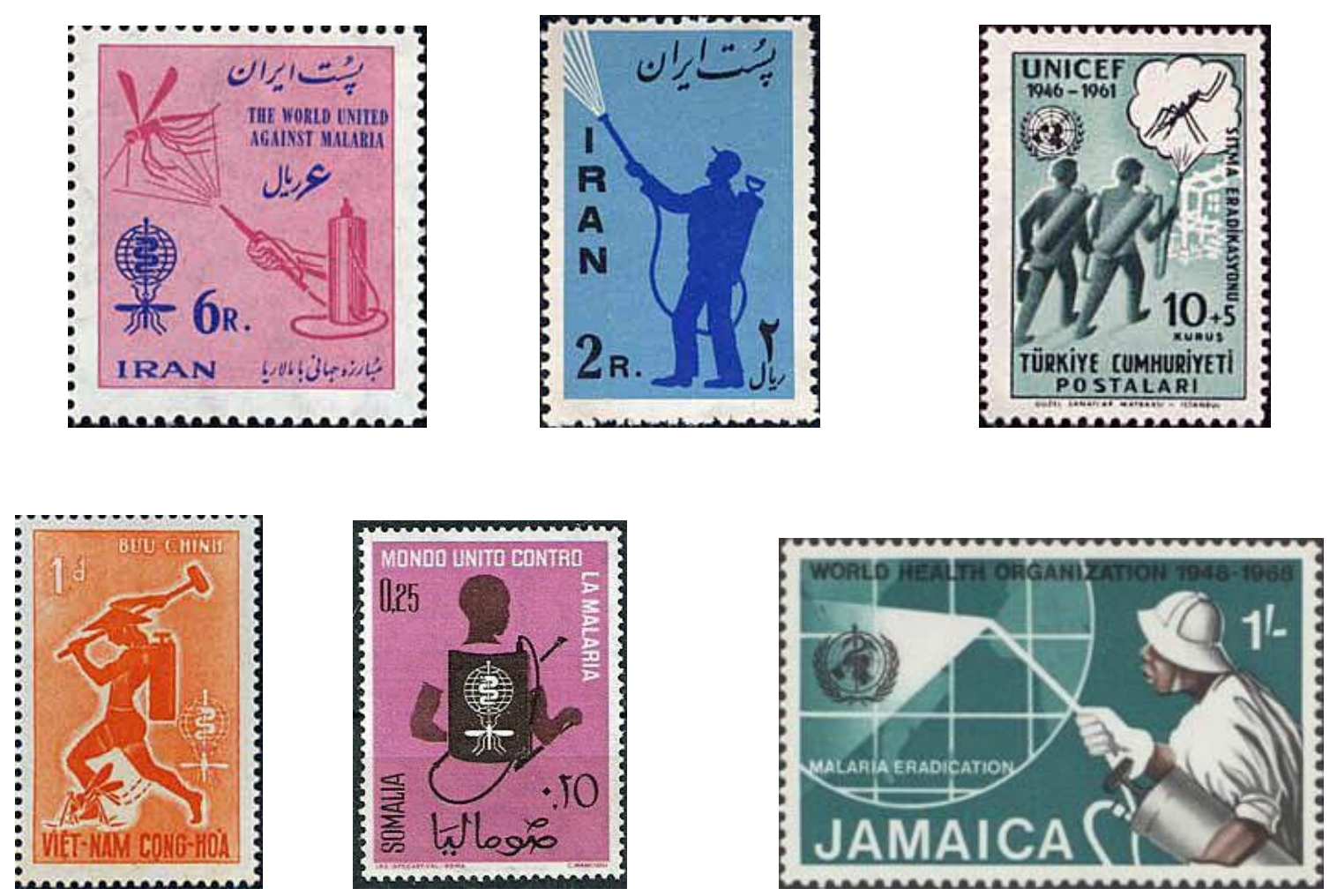

Figure 33. A wide selection of stamps directed at elimination of the mosquito by insecticide spraying. Nigeria (2), Iran (2), Turkey, Vietnam, Somalia and Jamaica.

\section{Malarial nets and insecticide impregnation}

The mosquito net dates from the $18^{\text {th }}$ century and was introduced to protect the sleeper from bites and stings from the insects at night and prevent subsequent infection. The mesh of the net must be fine enough to exclude the mosquito yet large enough to allow proper ventilation. From the 1980 s onwards, mosquito nets were treated with insecticides and these proved to be twice as effective as non-treated nets. They not only offered protection but repelled the insects and were also able to kill them. DDT was not used but another insecticide, permithen, proved to be very effective. ${ }^{88}$ There was encouragement for the wider use of nets during the campaign to complement the eradication process (see figure $34)$.

\footnotetext{
${ }^{88}$ Insect-treated bed nets, Centres for Disease Control, USA. https://www.cdc.gov/malaria/malaria worldwide/reduction/itn.html accessed 16/6/2020.
} 


\section{Effective treatment regimens and surveillance}

The main thrust of the treatment regimens was to establish a standard protocol and to adhere to these agreed plans. Surveillance was in four stages: preparation, attack, consolidation and maintenance. In terms of stamp usage, few countries used their stamps to encourage adherence or education regarding therapy. Three examples of this type of use are shown in figure 35.
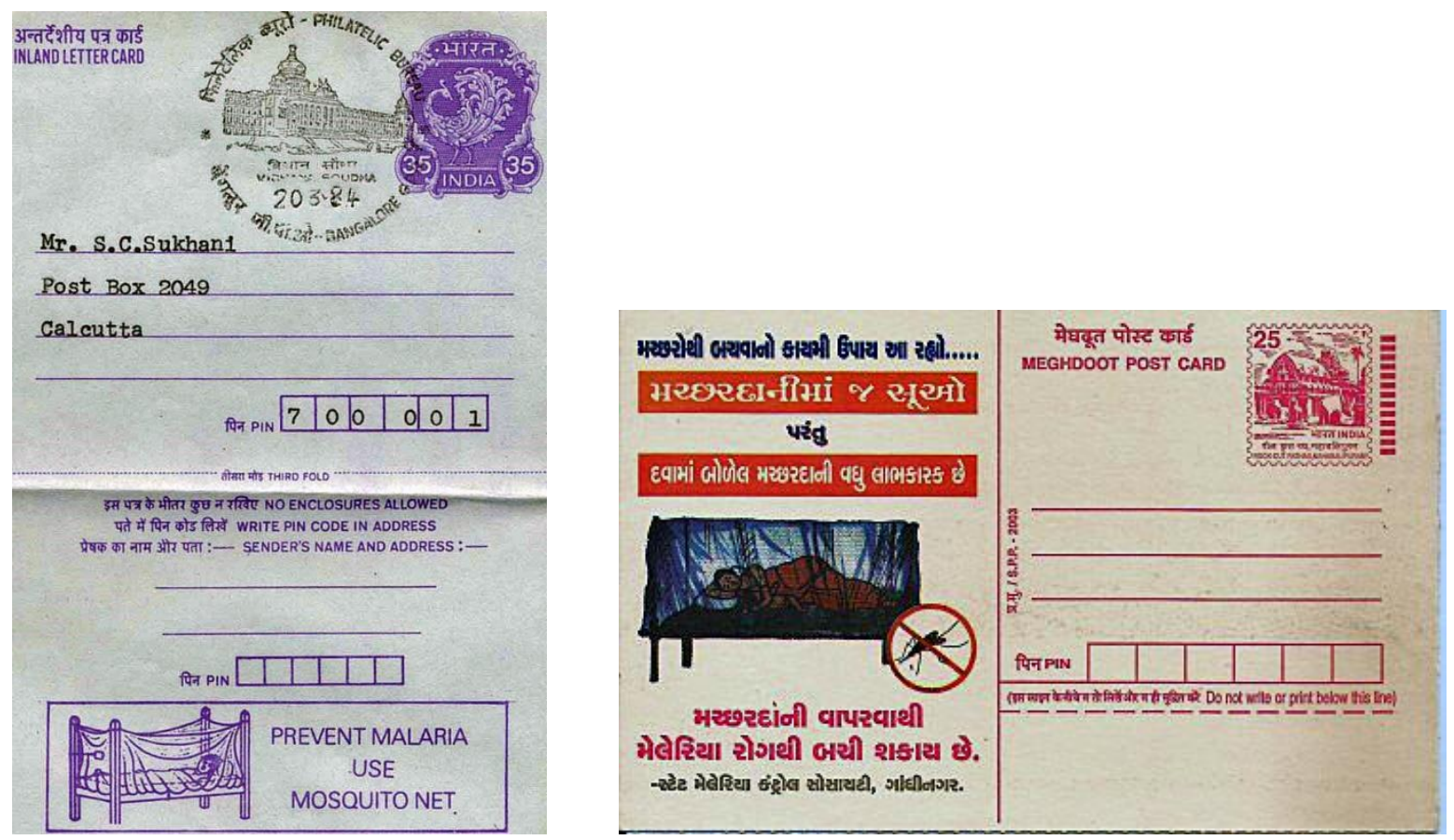

Letter card and Meghdoot postcard from urging use of malarial nets, India.
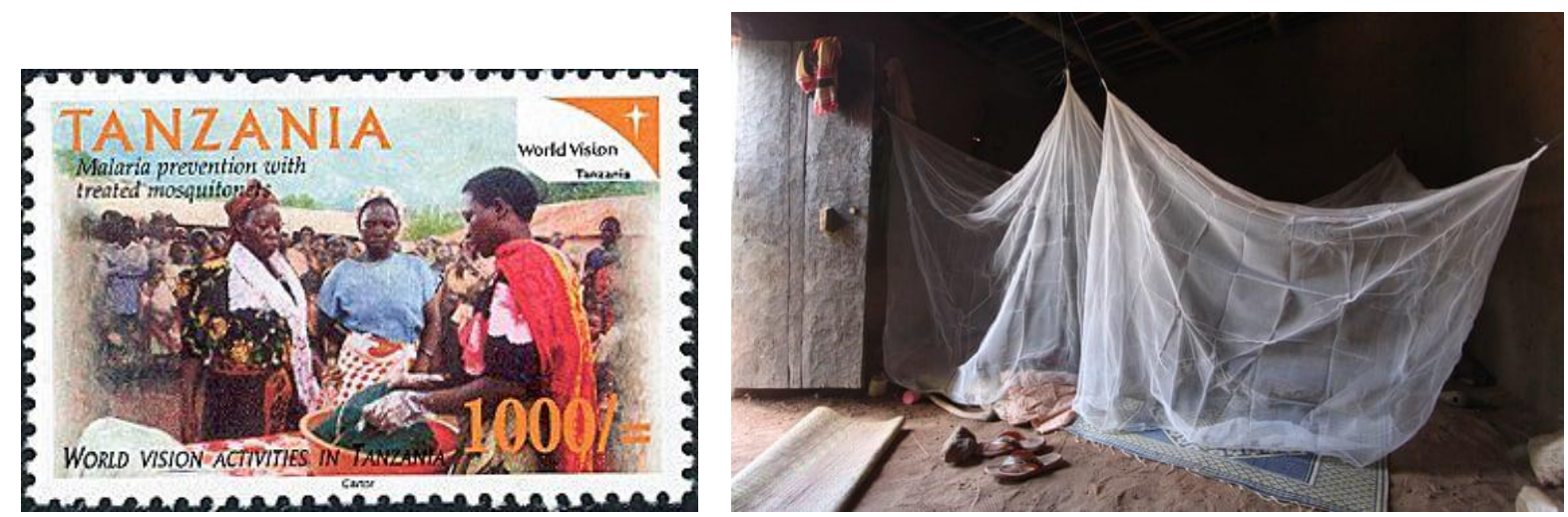

Figure 34. The purchase of mosquito nets in the market place and their use by an African family. Tanzania stamp and photograph, source: The Guardian, August 2018. ${ }^{89}$

\footnotetext{
${ }^{89}$ These examples are for demonstration only and were not issued during the international campaign specifically.
} 

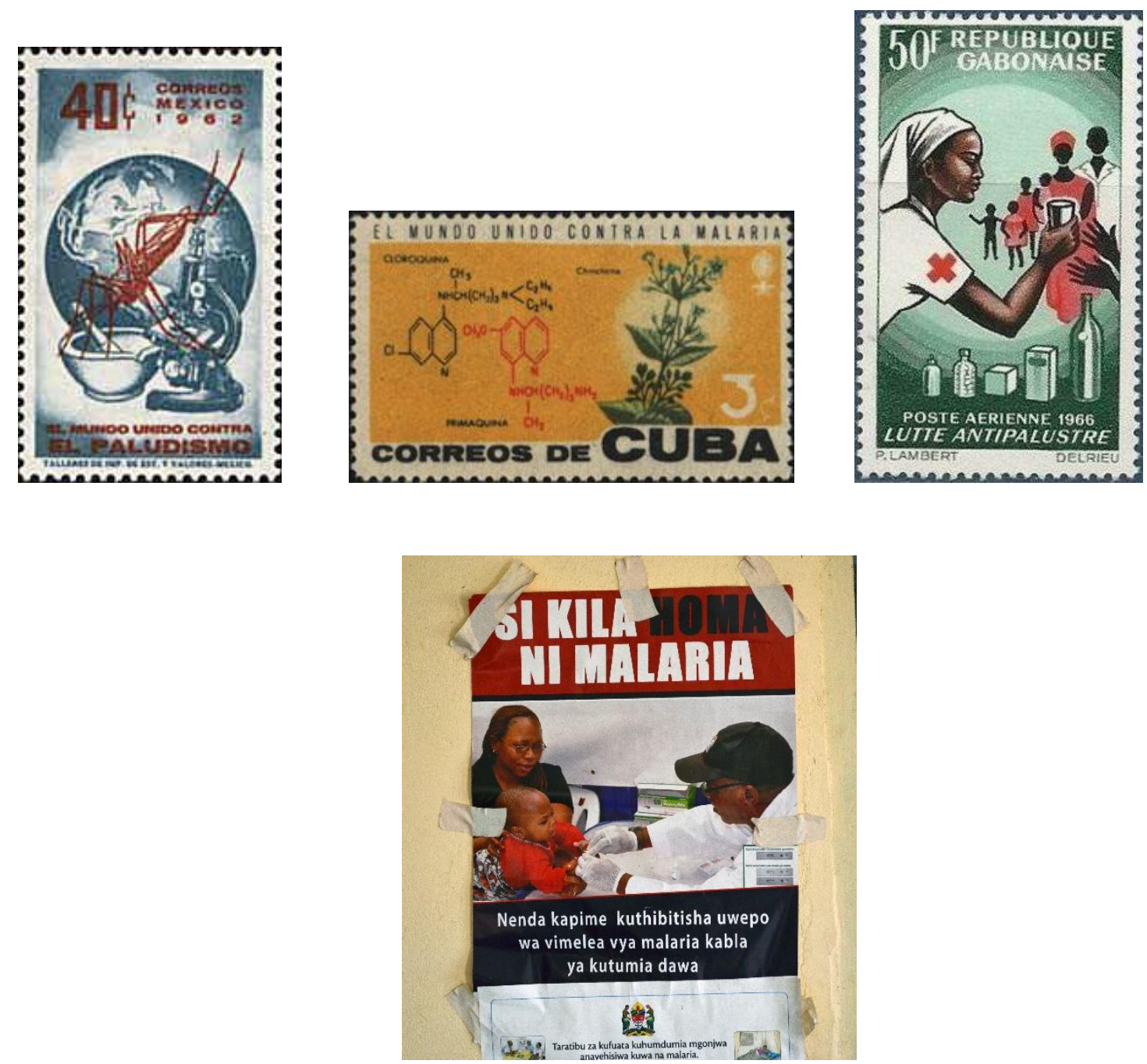

Figure 35. Diagnosis and treatment alluded to in the Mexican stamp of 1963. The Cuban postage stamp depicts a chinchona plant and the chemical formulas for chloroquine and primaquine, medications used to treat malaria. Treatment adherence is referred to in the Gabon stamp of 1966. Photograph: Malaria clinic, Kilwa, Tanzania, photo by the author.

Two other issues and a slogan relating to the malaria campaign are notable. The issue from the Panama Canal Zone while not especially, health promoting, does recall that malaria has a special significance in the Canal Zone as it undermined the French canal building effort of the 1880 s and 1890s. Interestingly, in the Canal Zone, the anti-mosquito campaign of the American Construction Period (1904-1914) resulted in the eradication of the even more dreaded yellow fever, although malaria still has not been totally eradicated from the Isthmus of Panama (figure 36)..$^{90}$ The Ethiopian stamps are the only set to stress the

\footnotetext{
${ }^{90}$ Canal Zone malaria:

https://postalmuseum.si.edu/exhibition/canal-zone-stamps-airmail-issues/7-cent-malariaeradication accessed 17/6/2020.
} 
international aspects of the campaign by an image. There was a pilot study in Ethiopia during 1955 and prior to the onset of the world eradication programme, as prior to 1939, three million people suffered from the disease each year in Ethiopia. The government of the country donated $20 \%$ of the revenue obtained from the sales of the malaria stamps shown in figure 36 , to the WHO to help fund the programme. Finally, an example of the slogan used by many countries who did not produce a dedicated stamp for the anti-malarial campaign is shown. This particular example is from Canada, but all others carried the same message in the appropriate national language.
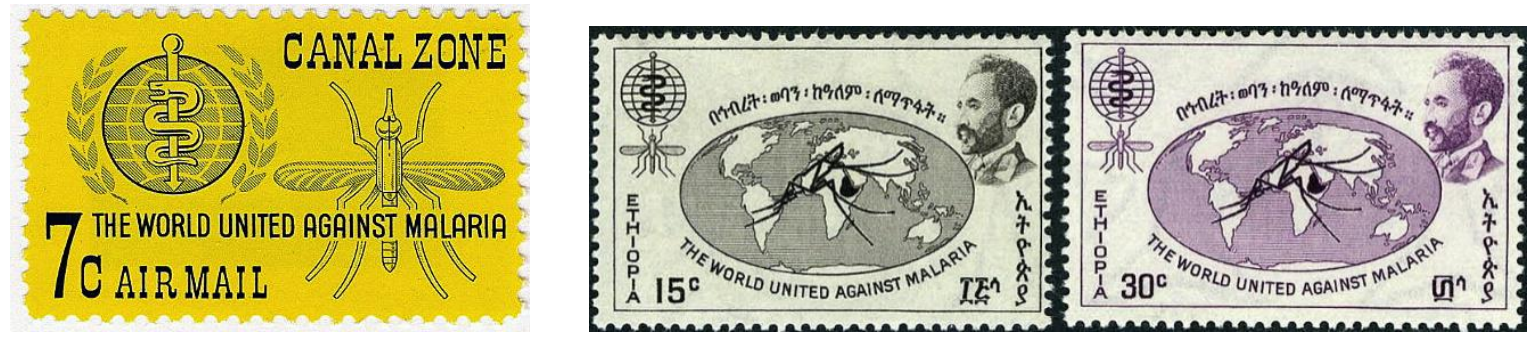

Figure 36. Panama Canal Zone issue of 1962, two of three stamps issued by Ethiopia, showing a world map with a mosquito superimposed.
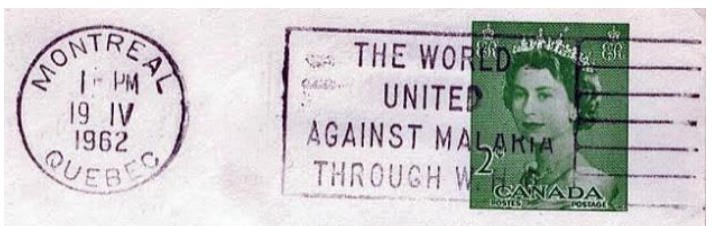

Figure 37.

Slogan stamp cancellation "The world united against malaria through WHO", 1962.

The campaign was not successful in its aim to eradicate malaria. There are many reasons given for this, among these are the emergence of drug resistance by the plasmodium and resistance of the insects to DDT, the onset of wars, population movements, and the requirement for the participation of the populations over a long term made continuing the campaign untenable. Sustained funding from donor countries also faltered and not enough attention was paid to newer nation's economies, their diversity and post-colonial economics. The programme was abandoned in $1969 .{ }^{91}$

\footnotetext{
91 José Najara, Matiana Gonzalez-Silva, Pedro Alonso, "Some lessons for the future from the Global Malarial Eradication Programme (1955-1969)", PLOS Medicine, 2011; 8: e1000412.

José Najara, "Malaria and the work of the WHO", Bulletin of the World Health Organisation, 1989; 67: 229243.

Arthur Brown, "Personal experience in the malarial eradication campaign, 1955-1962", Journal of the Royal
} 
To date this chapter has shown that while the attempts to eradicate malaria worldwide had failed because of the reasons listed above, there was a unique success in terms of health promotion on a global scale. For the first time, there was a worldwide response to the use of postage stamps to inform, advertise and promote a health programme and this was supported by over 140 countries and territories. The term 'omnibus' issue has been coined for such widespread use of themed stamps. ${ }^{92}$ There was a wide range of formats and several examples of these have been presented. Was this use of stamps successful during the campaign? The next section of this chapter strongly suggests that it was as there has been continued use of postage stamps to promote health knowledge about malaria during the first twenty years of the $21^{\text {st }}$ century.

\section{Malaria: health promotion stamp issues}

The approach of the WHO towards malaria eradication has changed. The current aim (20162030 ) is to reduce case incidence and mortality, hopefully to be as high as $90 \%$. In addition to have a country by country eradication programme and aim to achieve this in 35 countries by $2030 .{ }^{93}$ Many countries, still with malaria as a major health problem, have continued to promote postage stamps as a way to inform the population about their malaria strategies. Using the previous headings from the global campaign strategies, examples are presented of the post-World Campaign use of stamps and the images portrayed and the formats used (see figures 38-39). The stamp of Nicaragua is of interest, as it carries a health promotion message in text about malaria on the back of the stamp and is of relevance, therefore, to the discussion in Chapter 2.2 about the use of text on health promotional stamps.

Society of Medicine, 2002; 95: 154-1962.

92 James Watson, The Stanley Gibbons book of stamps, Ringwood: Stanley Gibbons Publications Ltd., 1990: 107.

${ }^{93}$ World Health Organization, Global strategy for malaria, 2016-2030, Geneva: WHO Press: 2015. 
Elimination and Spraying
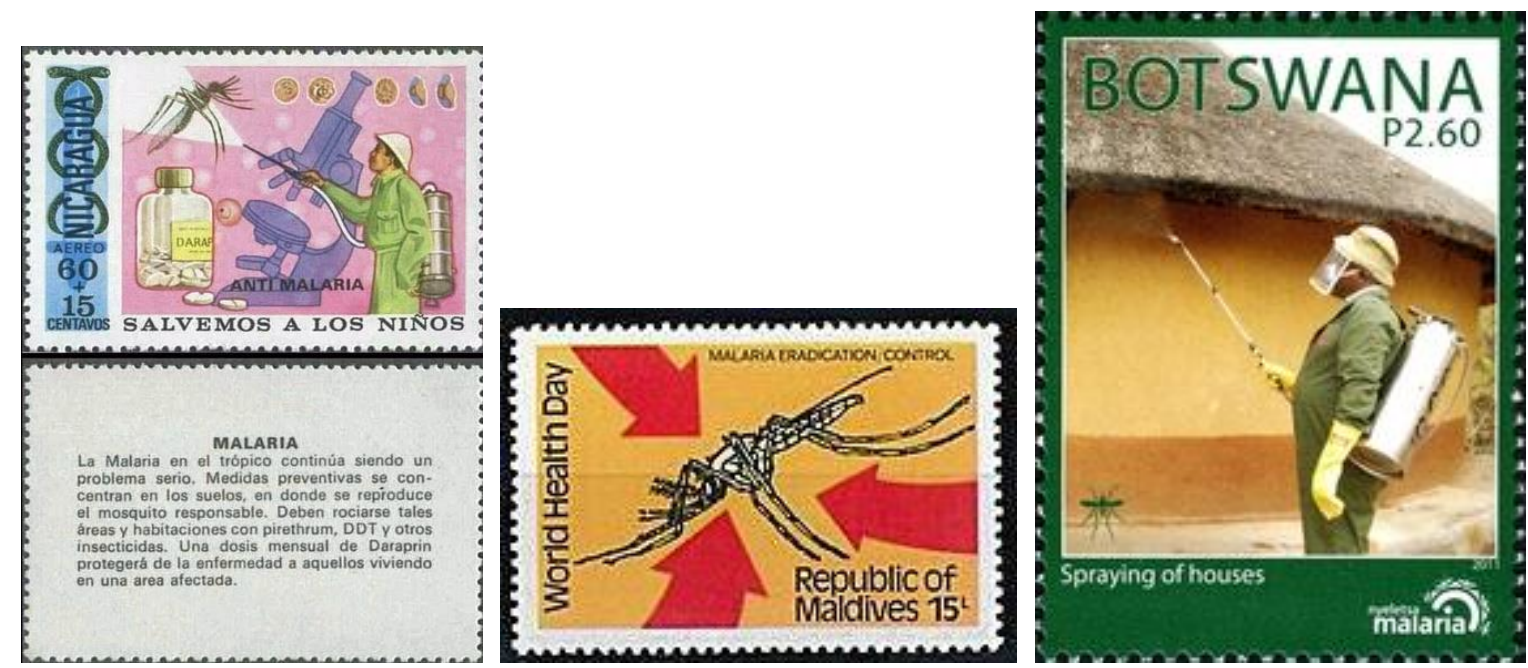

Additional stamps directed at mosquito elimination and insecticide spraying. Nicaragua, 1973, Maldives, 1980, Botswana, 2013.
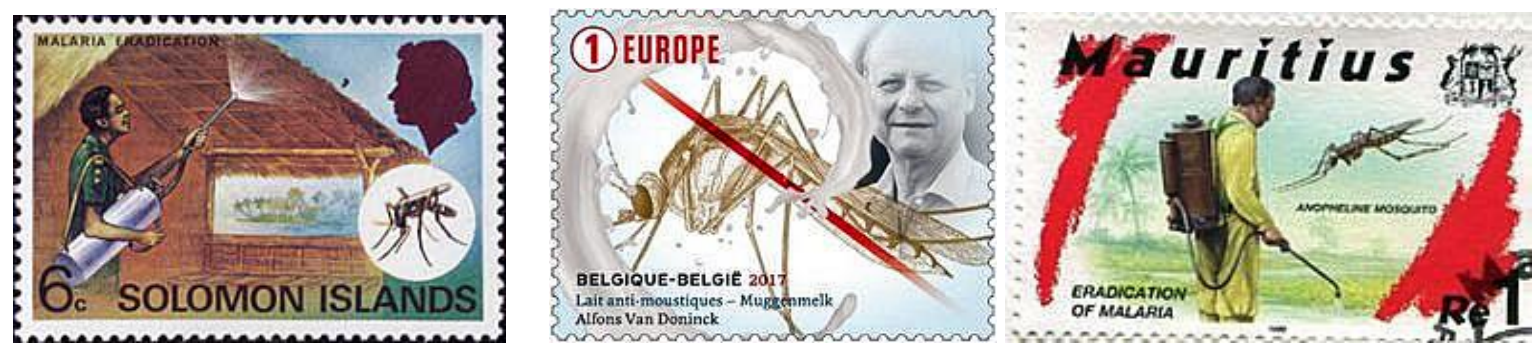

Figure 38. Malaria eradication: Solomon Islands, 1977, Belgium, 2017, Mauritius, 1999.

Malarial nets
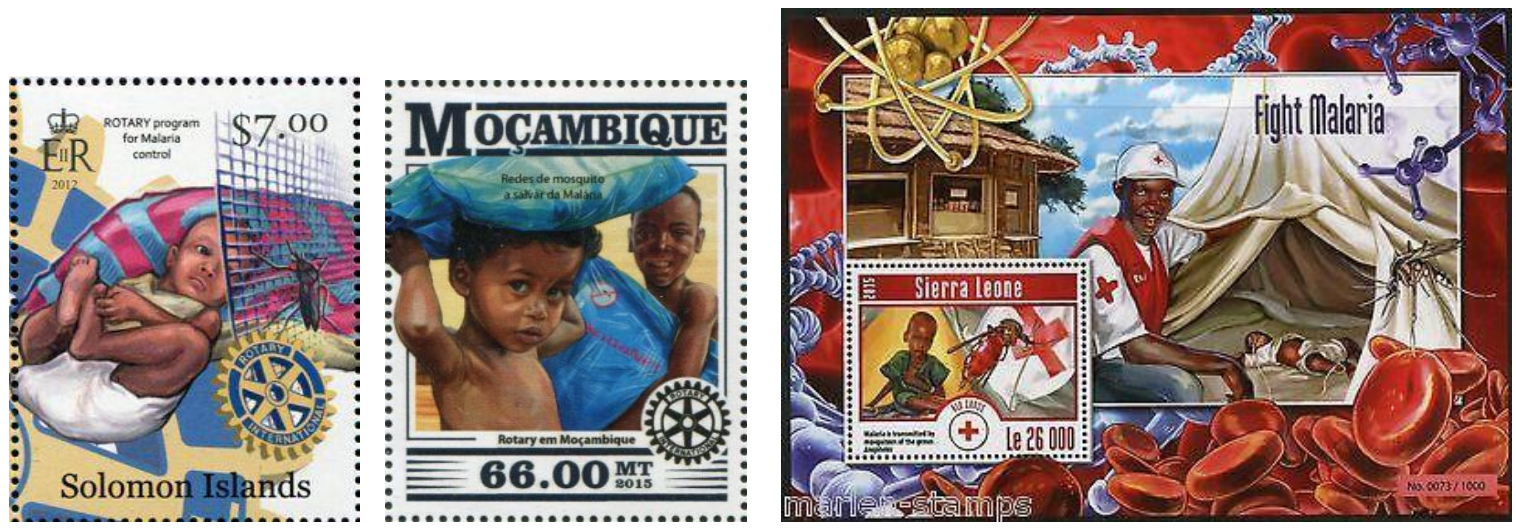

The support of malarial nets, Solomon Islands, 2012, Mozambique, 2015, Sierra Leone, 2015. 

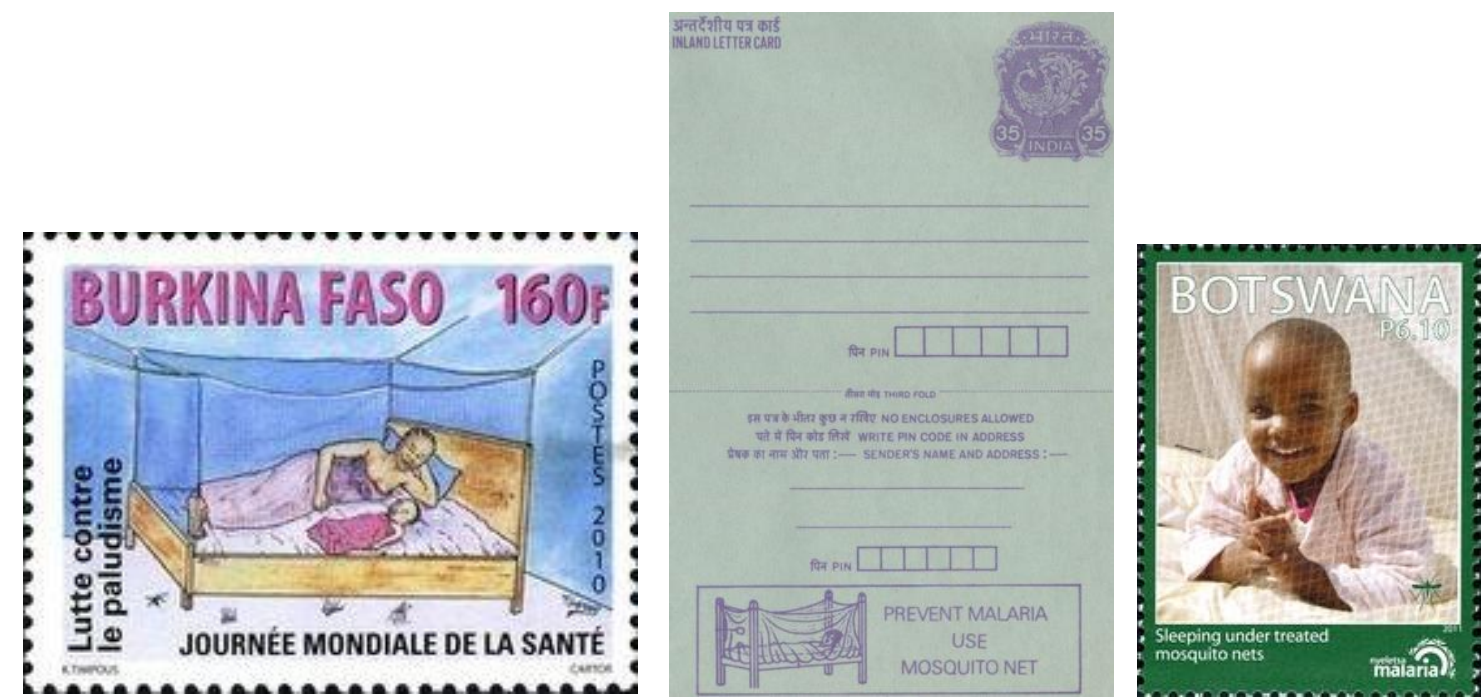

Clear encouragement to use nets, Burkina Faso, 2010, Indian inland letter and Botswana, 2011

Diagnosis and treatment of malaria

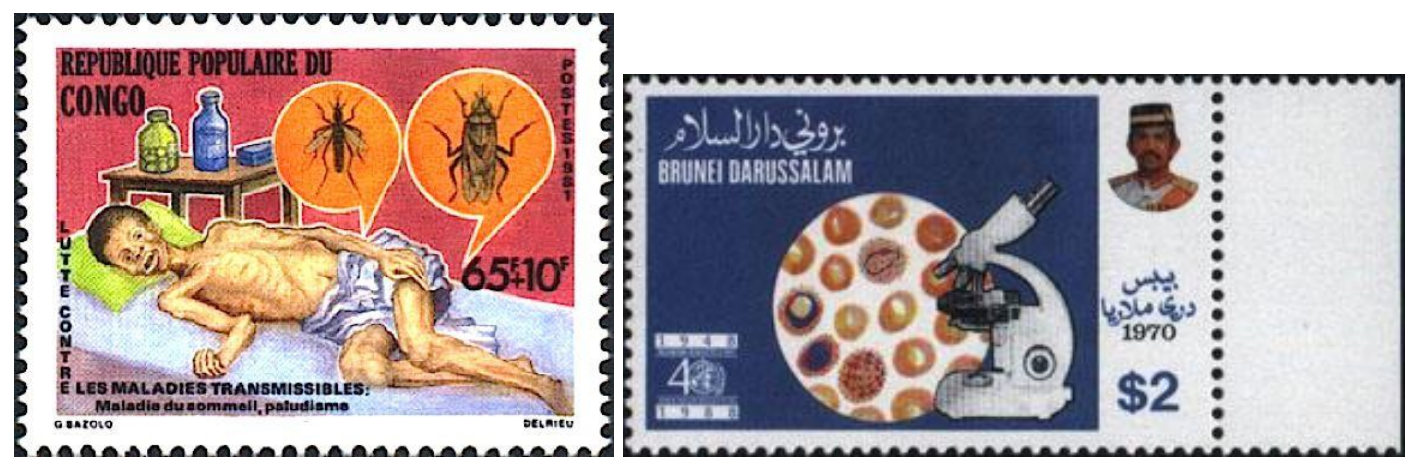

The necessity for rapid recognition and diagnosis of malaria is stressed in these and subsequent stamps. Congo, 1981 and Brunei, 1968.
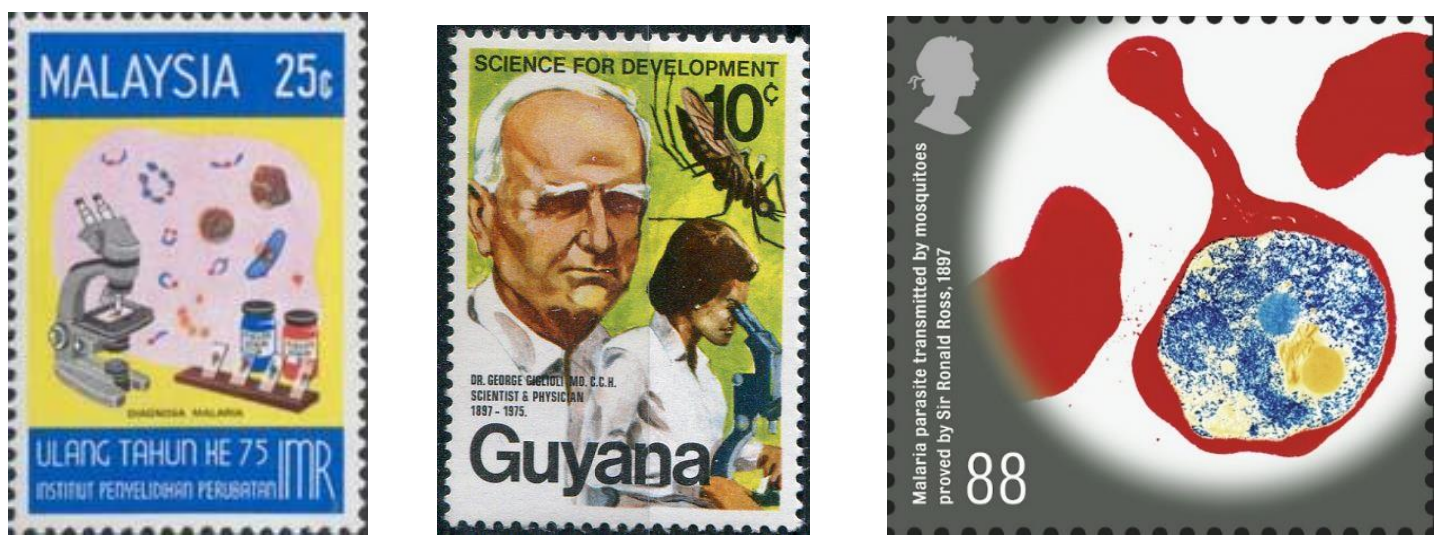

Rapid diagnosis by blood film examination, Malaysia (1975), Guyana (1978) and United Kingdom (2010). 

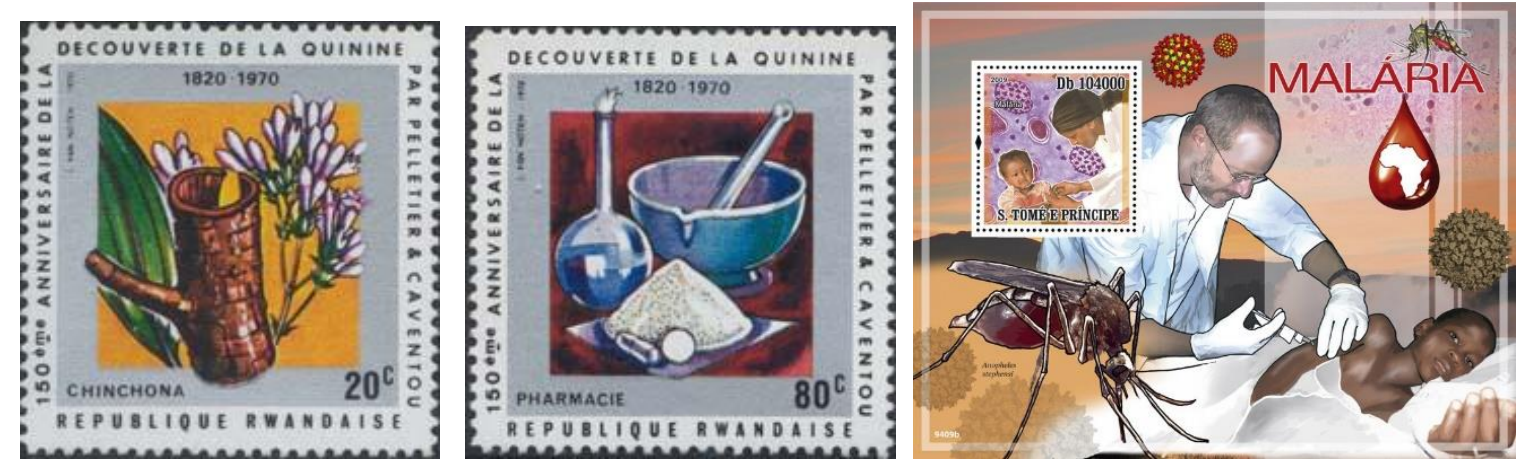

Discovery of quinine as the first treatment of malaria, Rwanda, 1970. Active treatment, St. Thomas and Prince Islands, 2009.
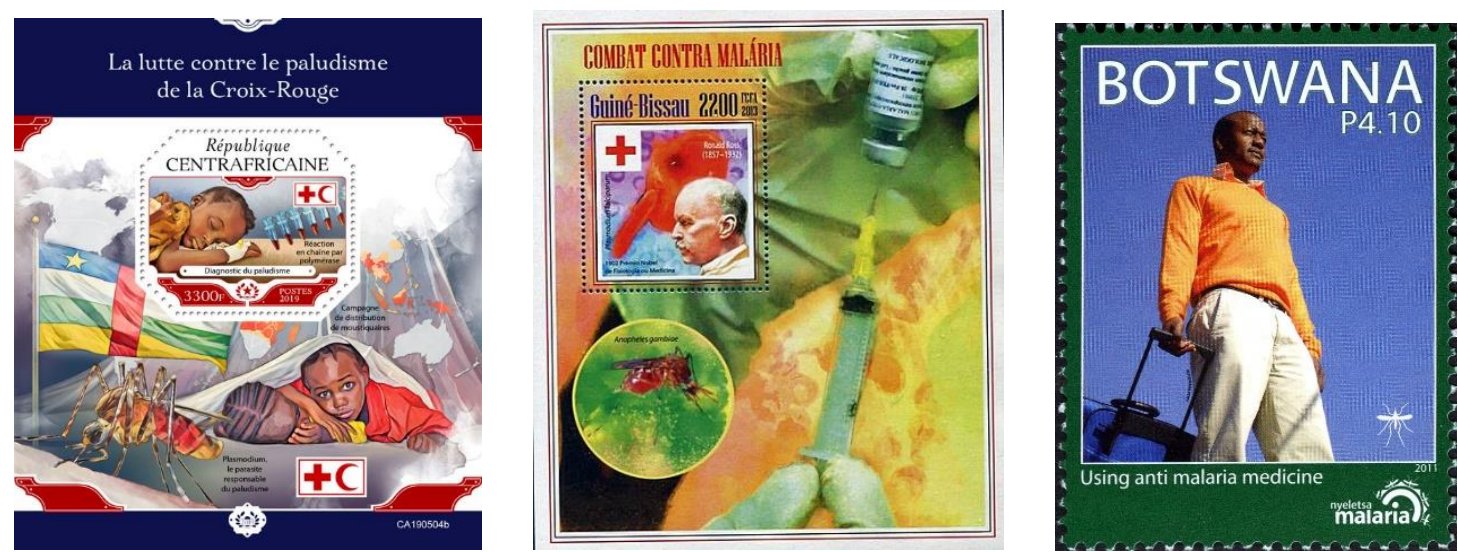

Active investigation and treatment against malaria, Central African Republic 2019, Guinea-Bissau, 2013, urging the use of prophylaxis, Botswana, 2011.
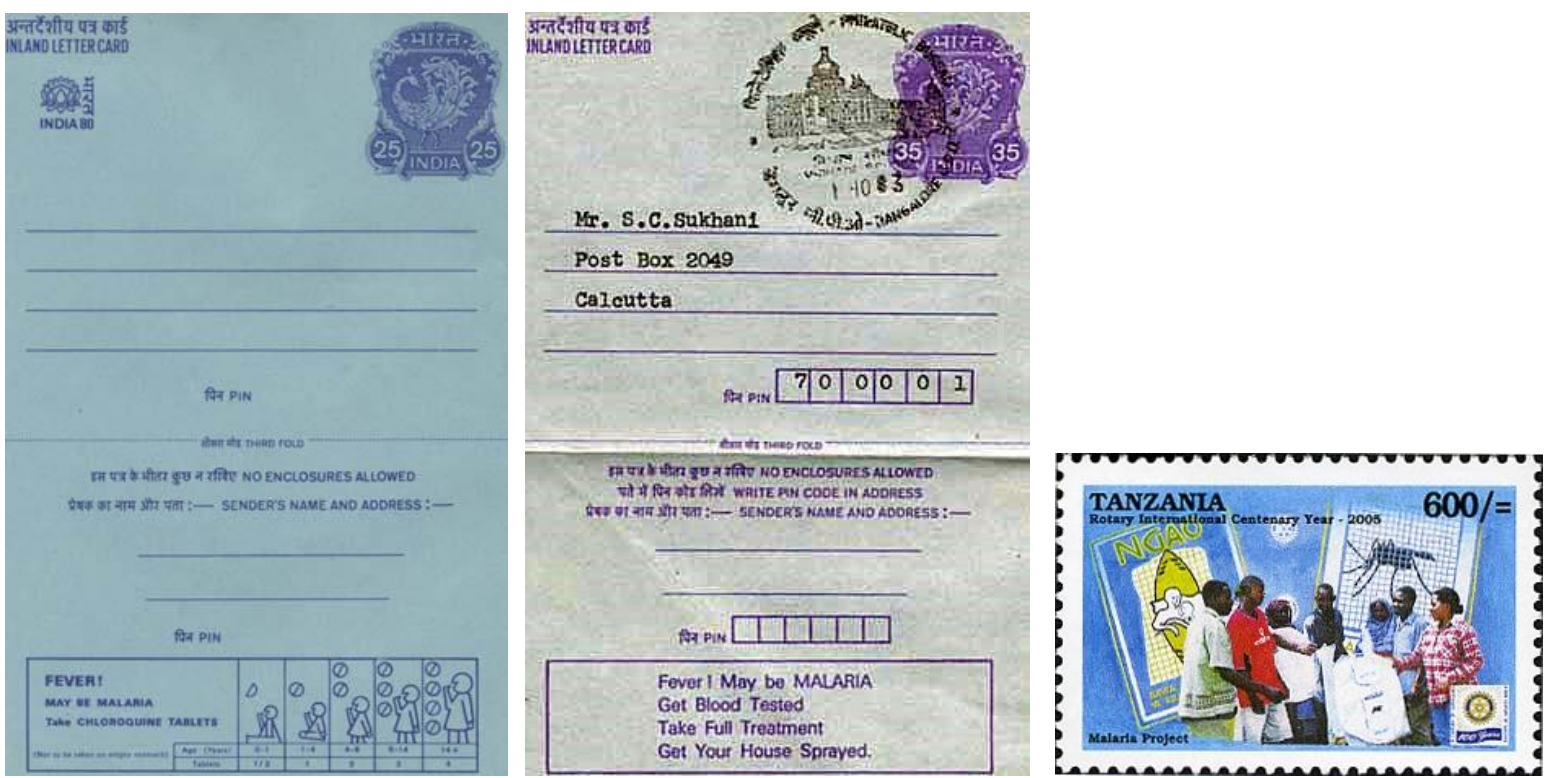

Figure 39 (1). Further examples of air mail letters with malarial advice, India, 1983, and active anti-malarial project, Tanzania, 2005. 
This chapter has provided many examples of how postage stamps have been used to promote the elimination of the mosquito and prevention and treatment of malaria. In doing so, it has gone some way in addressing the research question and demonstrating how international collaboration with postage stamp issues could support and strengthen the health campaign. The fact that the objectives and structure of the malaria campaign were flawed does not detract from the contribution made by stamps in its promotion. The campaign took place over fifty years ago but, its details have been included here as an example of a how many nations' actions were based upon the belief that issue of their stamps could in some way contribute to the campaign by education and promotion for a worthwhile cause. This contribution was other than in financial terms as none of the issued stamps were charity issues and none carried a surcharge. Indeed, there is visual evidence provided in the second part of the chapter that many of the countries involved in the early malaria campaign, have continued to use their postage stamps to inform and encourage their people to carry out anti-malarial activities and to use anti-malarial drugs for the acute illness and for prophylaxis. Many of these countries are in Africa, where malaria continues to cause a major burden on health resources, the economy in general and especially the lives of Africans throughout the continent. ${ }^{94}$ However, it must be noted that the production of postage stamps by many low income countries does form part of their sources of income, and not all the stamps produced reach the postal system of the country releasing them. ${ }^{95}$ Of relevance to this is the active use of a recent stamp of Sao Tome and Principe that supported a very successful anti-malarial campaign in that country and has resulted in an almost total elimination of the disease (Figure 39). ${ }^{96}$

'The World Against Malaria' campaign was not the first occasion that postage stamps were used to highlight the problems of malaria and to draw attention to the disease. The first dedicated stamp was issued by Mexico in 1939 with the illustrated theme of a large mosquito on the back of man who is on his knees - the burden of mankind (figure 40). ${ }^{97}$

\footnotetext{
${ }^{94}$ MA Groepe, PDF Urbach, KW Hlongwana et al., "Health promotion: from malaria control to elimination", South African Medical Journal, 2013; 103: 799-800.

95 Kenneth Dawson, Commerce or Commemoration? The role of the postage stamp in Great War remembrance, MA Thesis, Massey University, 2019.

${ }^{96}$ Pei-Wen Lee, Chia-Tai Liu, Herodes Sacramento Rampao, Virgilio E do Rosario, and Men-Fang Shaio, "Pre-elimination of malaria on the island of Príncipe", Malaria Journal, 2010; 9: 26.

97 Donald R. Johnson, “Malarial eradication and postage stamps”, Mosquito News, 1961; 21: 79-83.
} 
The fact that stamps have illustrated health promotion against malaria and have continued to do so, for over eighty years, is strong support for their positve role in this particular field.

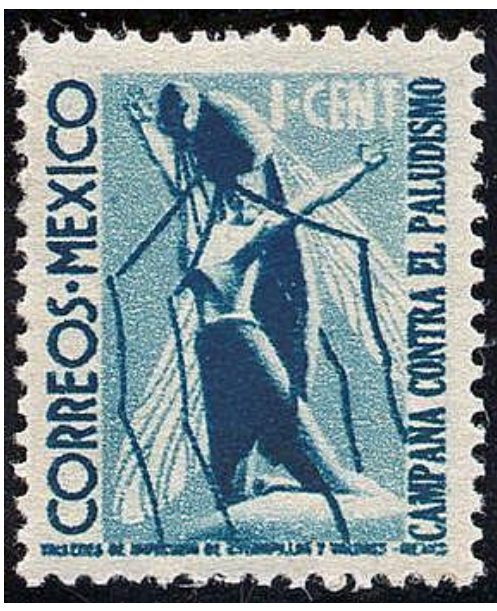

Figure 40.

Malaria mankind's burden, Mexico, 1939. 


\section{Chapter 5. Health Campaigns: the Role of the Postage Stamp as an Anti-smoking Health Messenger}

"A cigar has a fire at one end and a fool at the other".

Horace Greely 1811-1872 ${ }^{98}$

This chapter is designed to explore the way that postage stamps have been utilised in delivering anti-smoking health messages. The powerful impact they can present is demonstrated in the frontispiece and in figures 7 and 8. A graphical shock technique is an educational method that has often been used in anti-smoking propaganda and contrasts with that used in malarial education stamps (see Chapter 4). The aim in anti-smoking graphics is to use a 'persuasive' strategy to manipulate public perception around the harm of smoking. ${ }^{99}$ This is demonstrated in figure 41 by stamps and a graphic design using this approach. However, while this figure demonstrates the hard-hitting approach another technique is based upon the subliminal nature of graphic design in that certain postage stamps, where repeated visual contact with an image occurs, can result in the desired effect. ${ }^{100}$ This approach is probably conveyed in the first anti-smoking stamp from Czechoslovakia issued in 1976, at the time of the WHO campaign against smoking, and the first postal card issued by Poland in 1971 (figure 42). However, the majority of anti-smoking stamps depend upon their imagery to 'persuade' the viewer to change their ways. ${ }^{101}$ Rose has argued that the imagery is interpreted differently by different people and this is related to the 'culturally bound gaze'. ${ }^{102}$ It is proposed here that anti-smoking stamps present a simple message "If you smoke you may die or become very ill". This is hardly the type of message that requires deep cultural, international or historical interpretation as suggested by Kearns et al. ${ }^{103}$

\footnotetext{
98 Horace Greely: https://www.quotetab.com/quotes/by-horace-greeley accessed 9/7/2020

${ }^{99}$ Can Graphic Design Save Your Life?

https://www.itsnicethat.com/news/can-graphic-design-save-your-life-wellcome-trust-exhibitionlucienne-roberts-rebecca-wright accessed 25/6/2020

${ }^{100}$ Harlan Strauss, Politics, psychology and the postage stamp, Pennsylvania: American Philatelic Society, 1975;178-179.

${ }^{101}$ D. Zeigler, "Post-communist eastern Europe and the cartography of independence", Political Geography, 2002; 21: 671-686.

102 G. Rose, Visual methodologies: introduction to the interpretation of visual materials, London: Sage Publications, 2001.

${ }^{103}$ Robin A. Kearns, Tara Coleman, Jonathan Edmeades, “New Zealand children's health stamps: ideological artefacts linking health and place", Social Science and Medicine, 2019; 227: 38-46.
} 
The international community perceived that postage stamps were a potent method of delivering an anti-smoking message. By 1994, fifty nations had put out anti-smoking stamps and the figure had risen to seventy by 2007 . Figure 43 reveals a steady increase, over time, While there was a marked rise in 1980-1, this was as a result of the annual World Health Day stamp having an anti-smoking theme as its focus.
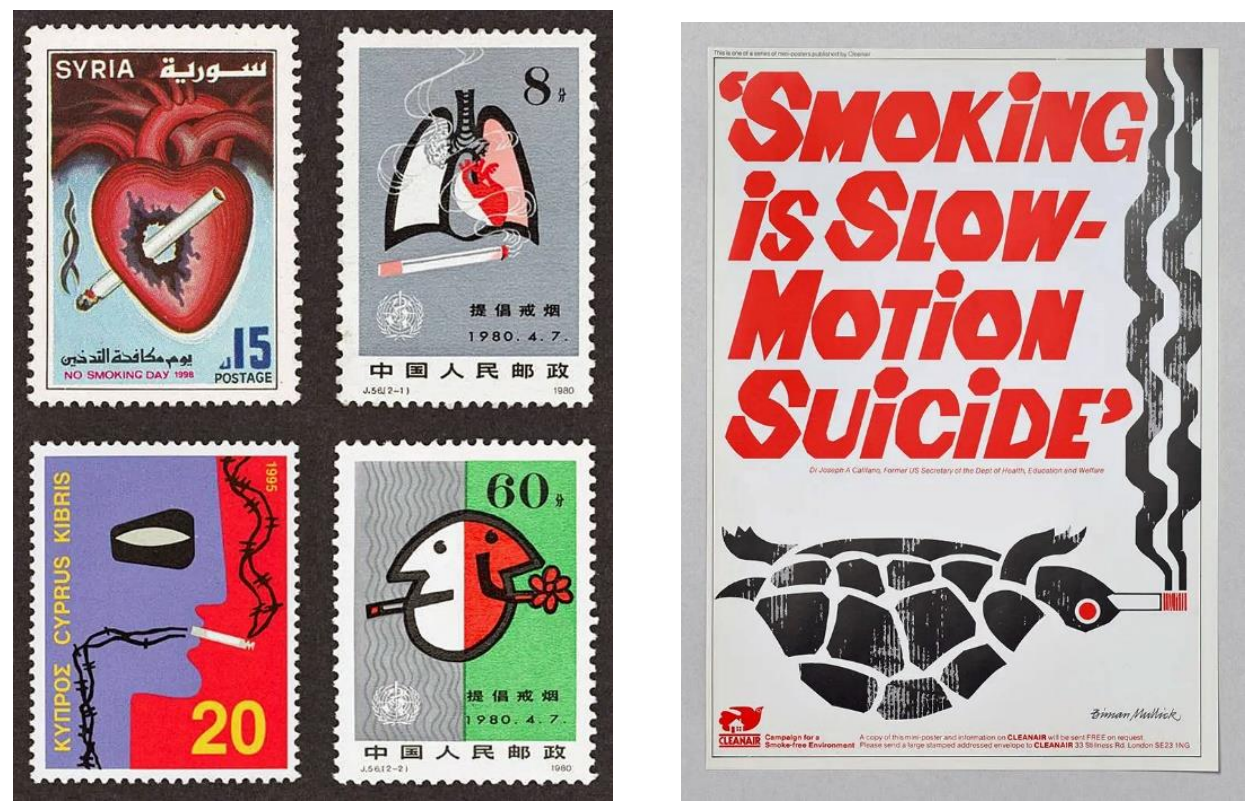

Figure 41. Anti-smoking stamps, Syria, 1998, China, 1980, Cyprus, 1995. Graphics advertisement, source: Biman Mullick/Cleanair "Smoking is slow-motion death".
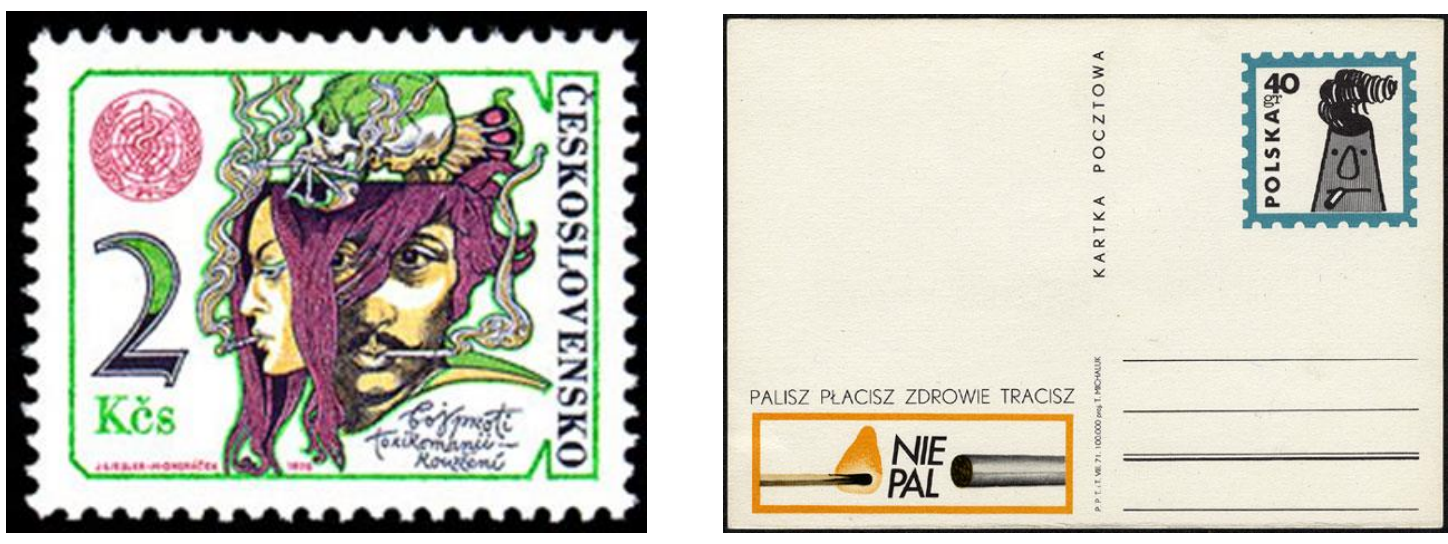

Figure 42. The first anti-smoking postage stamp, Czechoslovakia, 1975, designed by Josef Liester, and the first postal card with anti-smoking theme, Poland, 1971. 


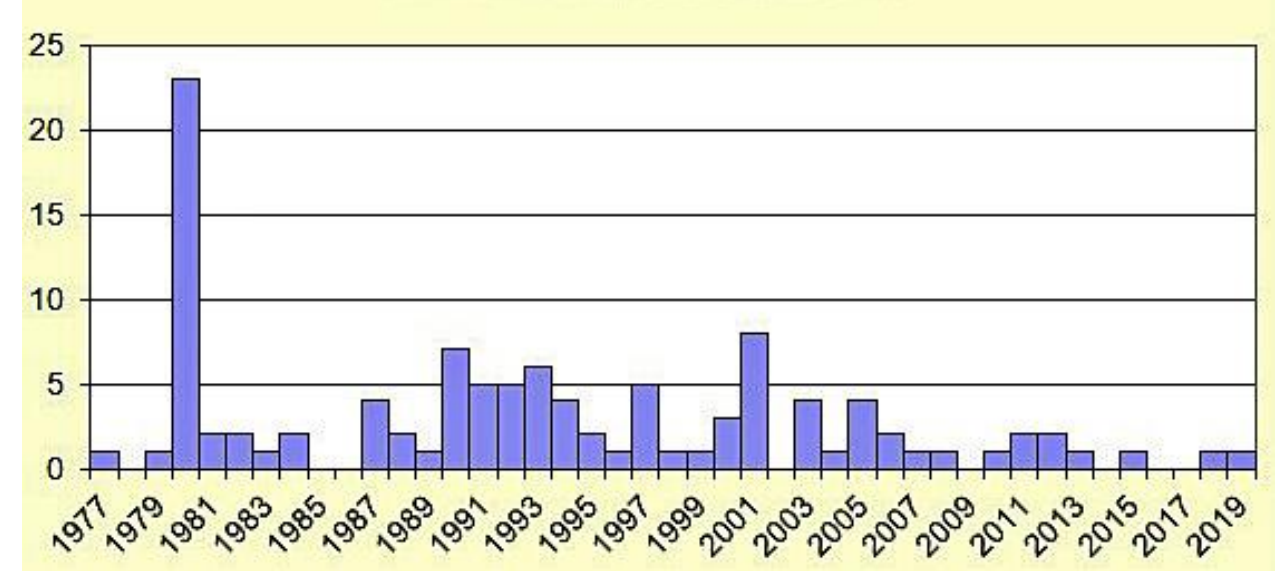

Figure 43. Number of anti-smoking stamps issued per year, 1977-2019. ${ }^{104}$

Further examples are presented in figure 44 to illustrate stamp presentation by a more aggressive image approach versus a more subtle one, often combined with a text message. There are two striking omissions in the list of countries that have issued stamps opposing tobacco use; the United States and the United Kingdom. This is perhaps not surprising when one considers that the United States is the world's major exporter of cigarettes and a grower of tobacco, while the United Kingdom exported over 11 million pounds sterling worth of tobacco goods in 2019. ${ }^{105}$ One could interpret this fact in that these respective governments value tobacco income over health costs.

While many governments have used the postage stamp to further their anti-smoking objectives, this raises the question as to whether the opposite holds true in that stamps have been issued to further the tobacco industry. Until 1978, there were more than ten countries who had issued stamps drawing attention to their tobacco industry. Surprisingly, in spite of all the adverse publicity about tobacco use there have been at least three issue about tobacco in the last seventeen years and one of these actually refers to the export of cigarettes from Austria (figure 45). The tobacco industry in Greece also received a boost

\footnotetext{
${ }^{104}$ Source of graph data: www.trussel.com/stamps/smoking/antismok.htm. accessed 29/6/2020.

105 James H. Lutschg, "Anti-tobacco postage stamps", Tobacco Control, 1992; 1: 5-6.
} 
with the issue of a postage stamp and the release of a pro-smoking card both also shown in figure 44 .

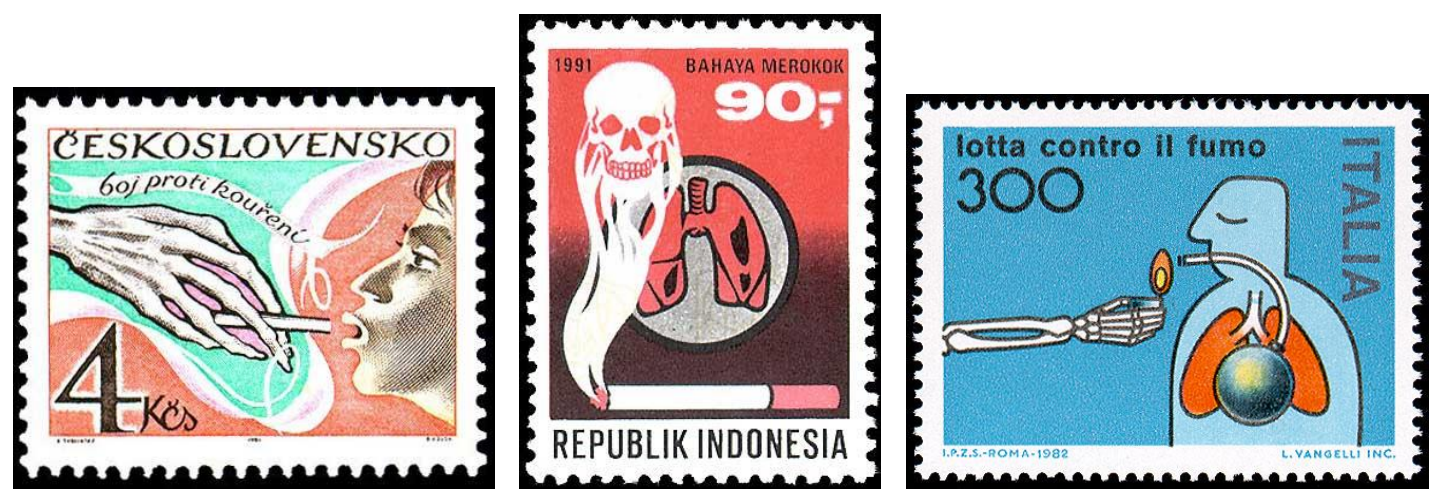

Figure 44. More dramatic use of images, Czechoslovakia, 1981, Indonesia, 1991, Italy, 1982.

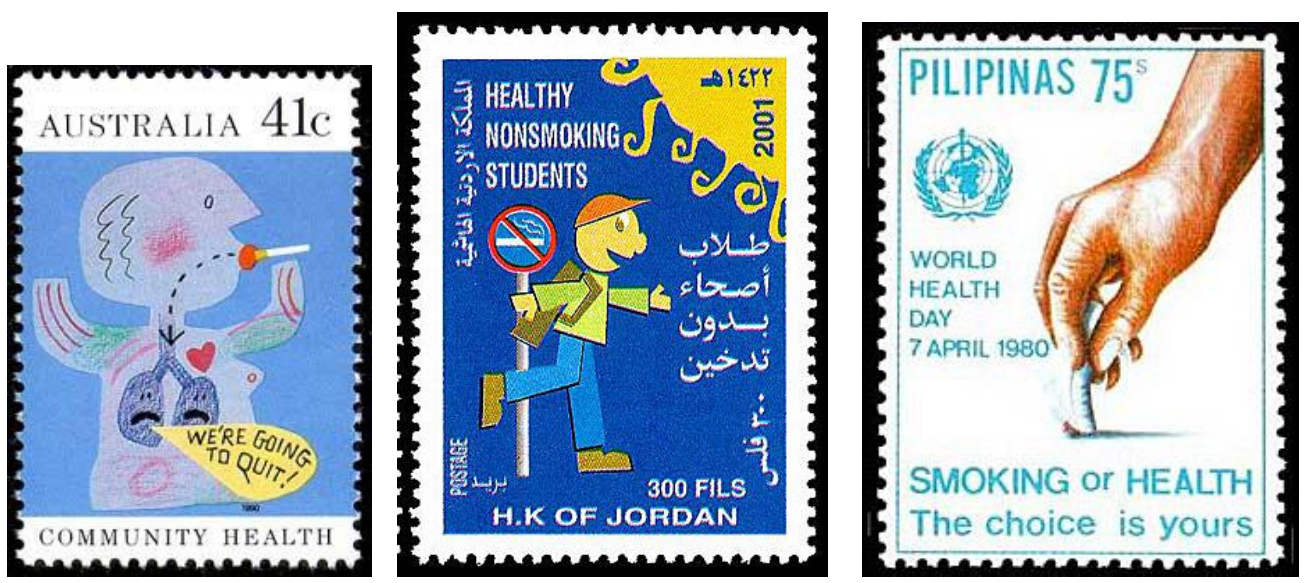

More subtle use of images with text, Australia, 1990, Jordan, 2001, Philippines, 1980. 


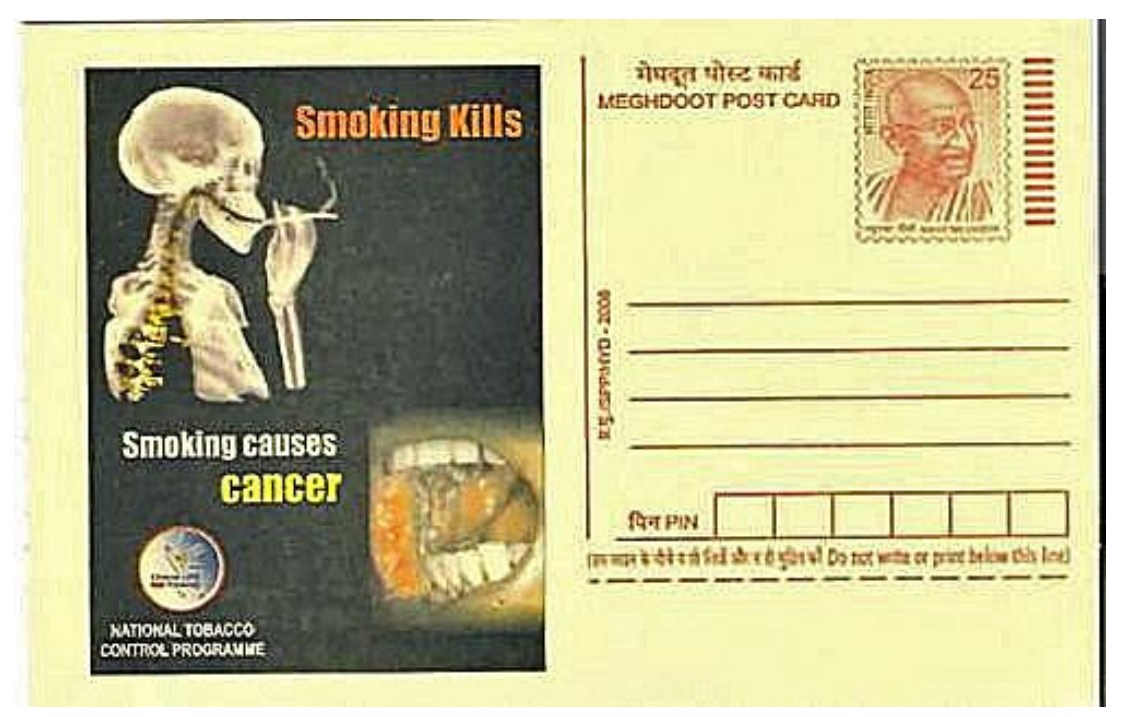

Anti-smoking meghdoot post card, India.
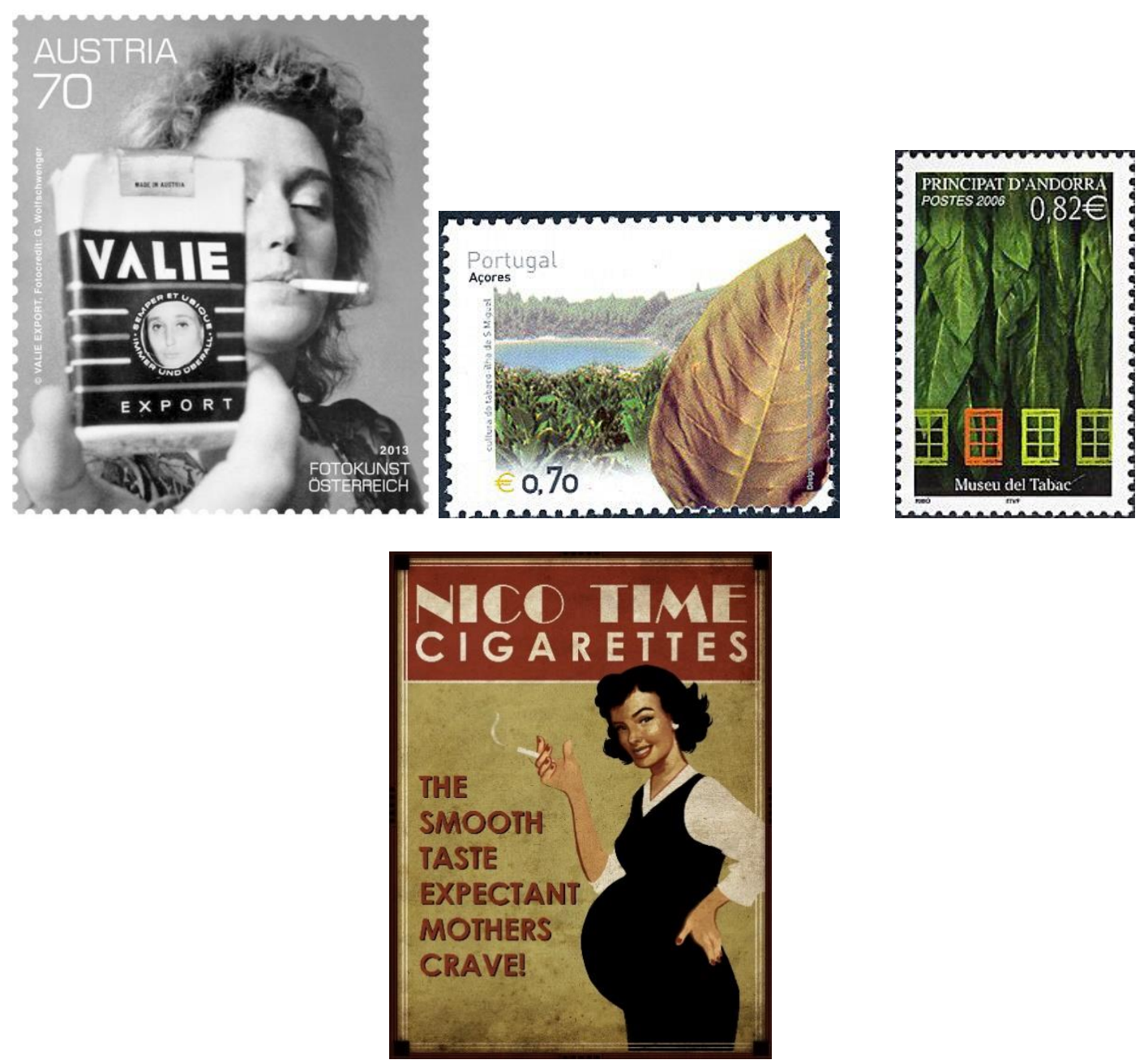

Austria, 2013, cigarette exports, Azores, tobacco growing, 2003, Andorra, 2006, tobacco museum, an early pro-smoking advertisement (source Pintrest). 

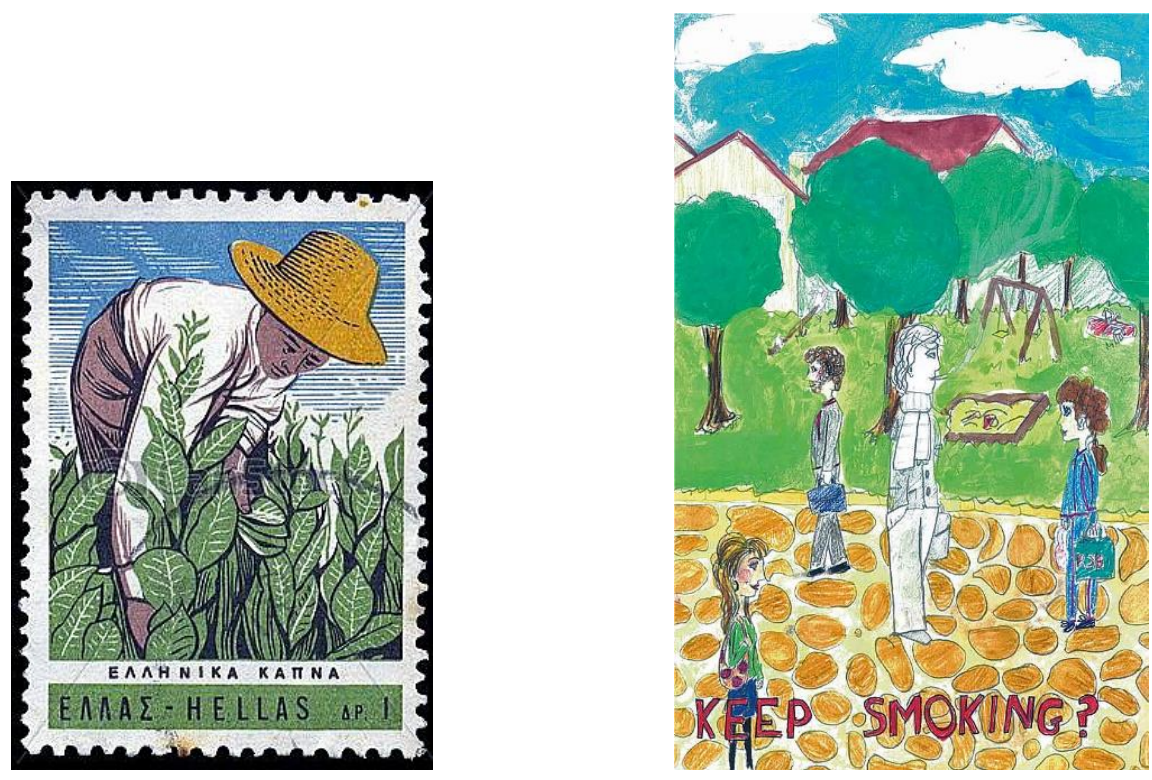

Figure 45. Promoting the Greek tobacco industry, Greece, 1966, Keep smoking card, source: The Greek Tobacco Epidemic, Harvard School of Medicine.

An interesting turn of events occurred with the issue of three stamps, by the USA, celebrating well-known Americans, James Dean (1996), Jackson Pollock (2010) and Robert Johnson (1994), the jazz guitarist. All three stamps were based upon original photographs of the individuals. The photographs and the subsequent stamps are shown in figure 46 , and it will be noted that the cigarette shown in the photograph has been removed in the final stamp design. When this was realised, there was an outcry from American smokers at this action, and this was based upon the fact that they perceived the removal of the cigarette as an encroachment on their civil liberties and an article appeared in their journal to this effect. (see figure 46).

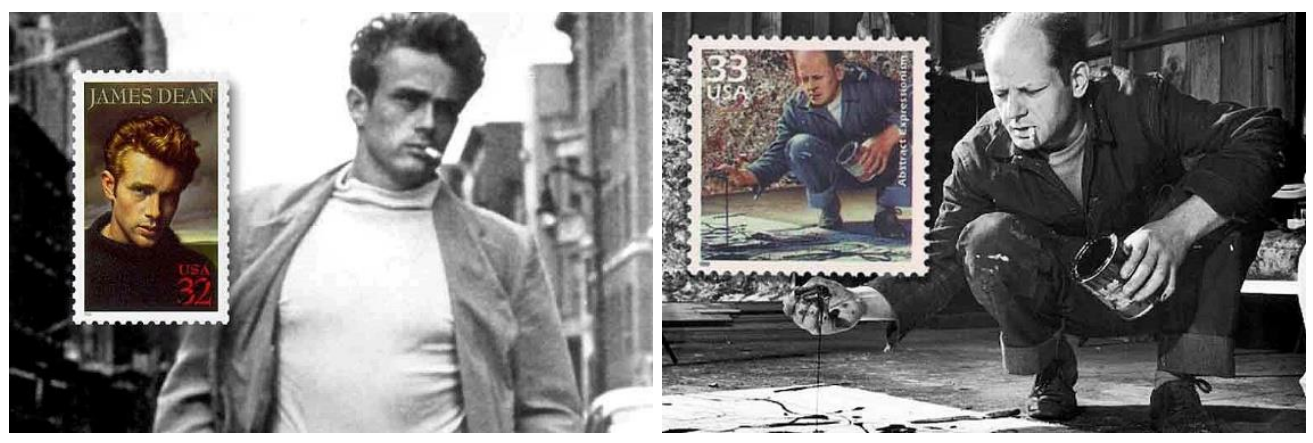




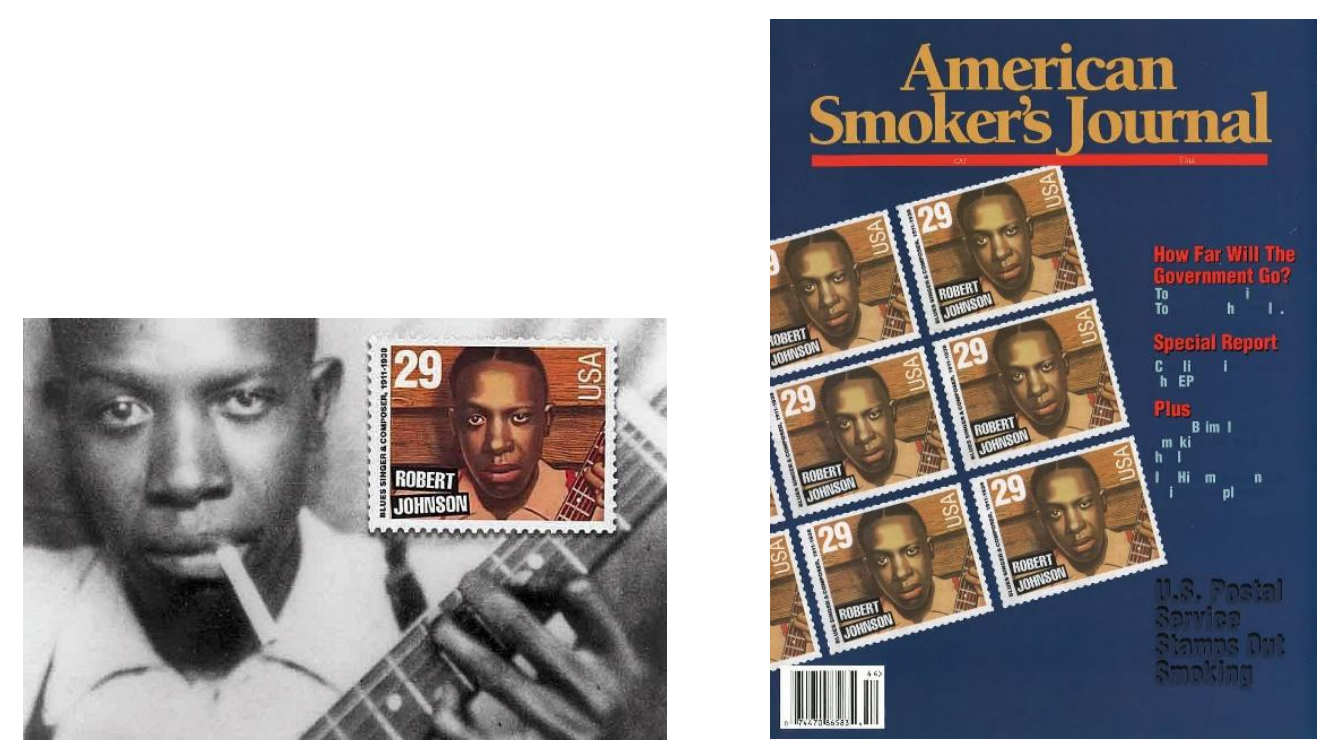

Figure 46. Original photographs and postage stamps based upon these photographs minus the cigarette. "How far will the government go?" from cover of the American Smoker's Journal. ${ }^{106}$

Internationally, there has been widespread use of stamp issues with anti-smoking images that utilise the potential secondary power of the postage stamp. The majority of these issues appear to have little intent to impart knowledge, but to use 'shock' tactics to alter personal behaviour. These stamps have stood alongside the well-established and widely displayed anti-smoking posters and the more recent use of cigarette packaging with graphic images of diseases associated with smoking. All of these media use the 'shock' or 'impact' approach to give their message.

Are these media images effective? There is good evidence that the use of graphic images on cigarette packages enhance the effectiveness of the warning labels (figure 47). The images can cause a replacement of a positive association with smoking by a negative association. ${ }^{107}$ Further research into the effects of cigarette packet images has revealed that, for active smokers, that they induce emotions of disgust, disappointment and regret. For nonsmokers, their emotional impact results in concern for dying smokers and for the animal testing that has occurred in relation to smoking research. ${ }^{108}$ There is no obvious reason why these findings cannot be interpolated to posters and stamps. If anti-smoking stamps do

\footnotetext{
106 The University of Alabama - Stamp out smoking https://csts.ua.edu/stamps/airbrushed-stamps/ accessed 29/6/2020

107 Geoffrey Fong, David Hammond, Sara Hitchman, "The impact of pictures on the effectiveness of tobacco warnings". Bulletin of the World Health Organization, 2009; 87: 640-643.

108 Philip Gendall, Janet Hoek, Katherina Gendall, "Evaluating the emotional impact of warning images on young adult smokers and susceptible non-smokers", Journal of Health Communication, 2018; 23: 291-298.
} 
have this effect, then there is every reason to continue with them. Indeed, the most recent stamp issued from Vietnam in 2018 carries a health message as well as an image (figure 46).
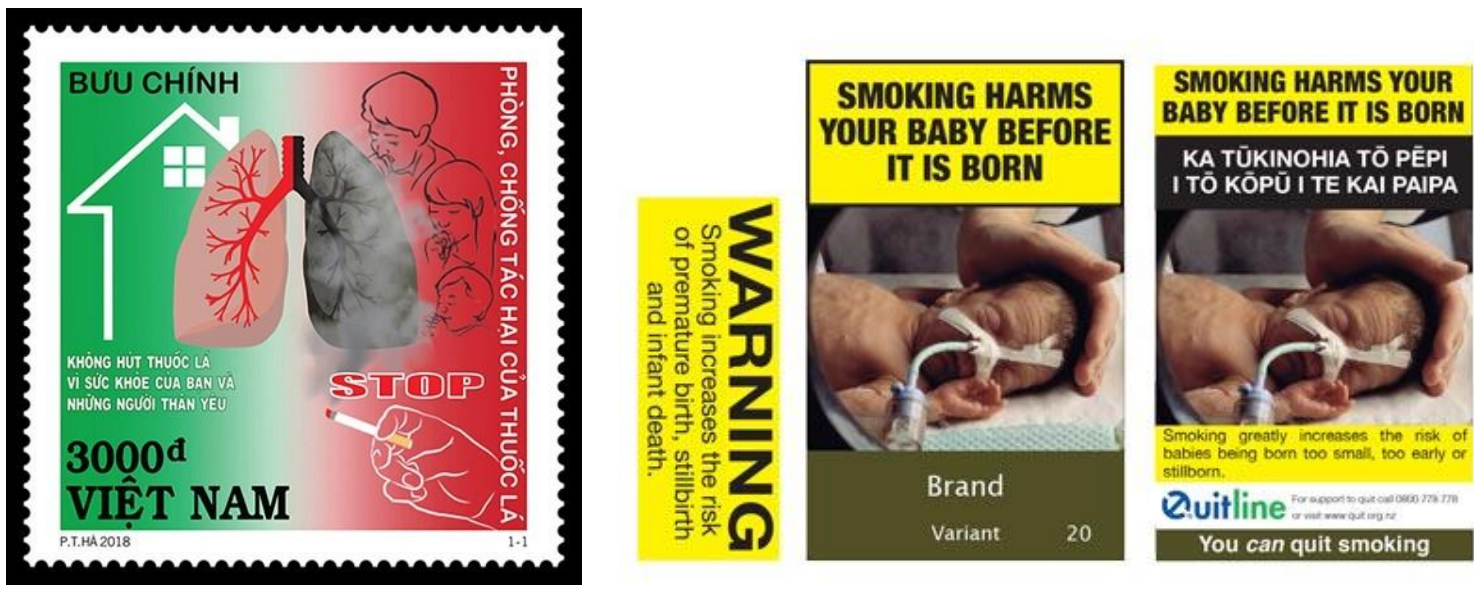

Figure 47. Vietnam, 2018 "Do not smoke for the health of you and your beloved ones", cigarette warning image, New Zealand. ${ }^{109}$

The images presented in this chapter are intended to form part of disease prevention campaigns and are not a health promotion activities per se. These themes will be developed and discussed in a later chapter. This chapter, in summary, has outlined the worldwide utilisation of postage stamps in campaigns to discourage cigarette and tobacco use. A large number of these issues were released as part of the World Health Day theme "Smoking or health the choice is yours" in 1980. The associated spate of releases is displayed in figure 43, but this figure also reveals that stamp production of themed stamps has continued through to 2019 and a recent release by Vietnam is illustrated in figure 46. This issue combines a text message with a visual one indicating smoking can harm not just the smoker but their family by passive inhalation. Several countries have supported their tobacco industries by stamps until the late 1970s when there was more awareness of the dangers of tobacco. A very

\footnotetext{
${ }^{109}$ Cigarette image: https://www.health.govt.nz/our-work/preventative-health-wellness/tobaccocontrol/tobacco-standardised-packaging/tobacco-packaging accessed 6/7/2020.
} 
surprising issue was that of Austria in 2013 (figure 45), which could be misinterpreted by many as supporting smoking.

Importance has been placed by most issuing authorities on graphic or even gruesome images to have impact and hopefully result in the required behavioural change. There is good research evidence that this type of approach is effective when used on the cigarette packaging itself and it would seem likely to have the same effect with stamps. Perhaps even more so, as non-smokers are exposed to the images on the stamps also. While the overwhelming number of stamps have had graphic images, the Japanese Post released a more subtle contribution when Japan hosted the Sixth International Smoking and Health Conference in 1987. Figure 48 shows this stamp and contrasts the pink complexion of the Queen holding a bird, with the blue complexion of the King holding a cigarette in his hand.
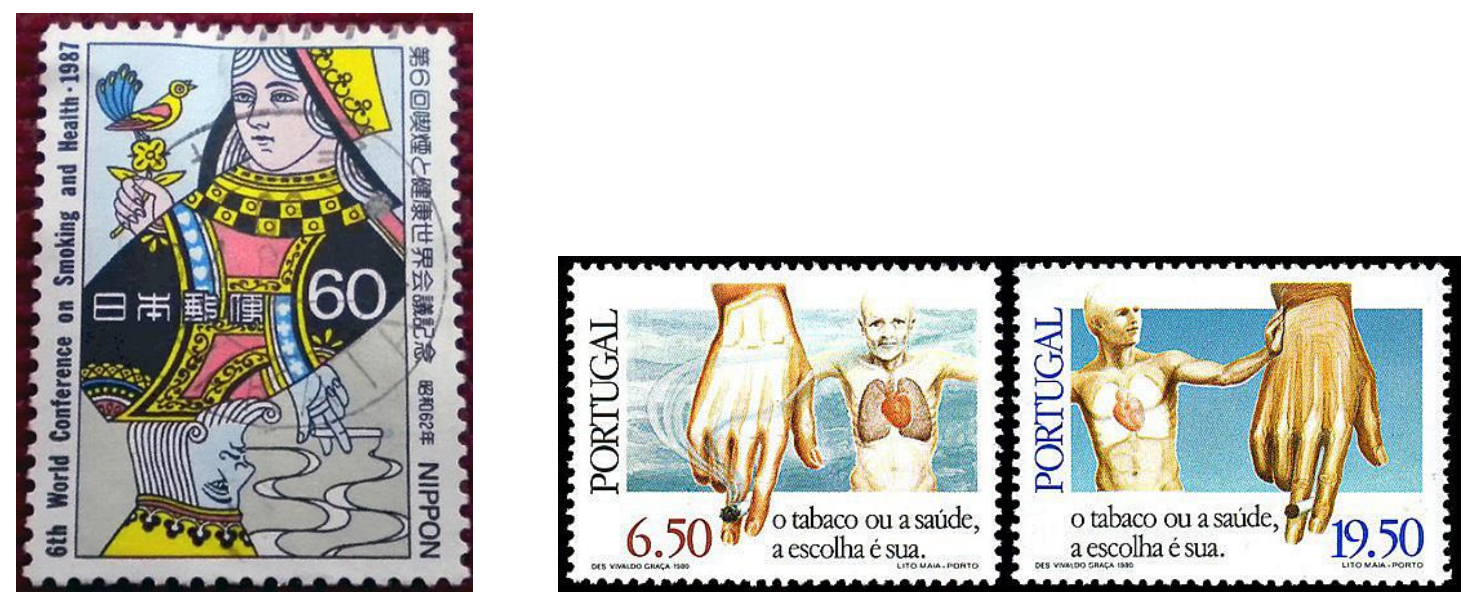

Figure 48. Contrasting anti-smoking images, Japan, 1987 and Portugal, 1980, a subtle versus a more graphic image presentation.

It is a striking observation that the USA and the UK have never issued anti-smoking postage stamps, especially as the United States issues on average over 80 new issues per year and in 2019 printed 16.5 billion stamps. ${ }^{110}$ This observation suggests national financial self-interest and a strong pro-smoking lobby at the expense of health. This is supported by the outcry that occurred by the removal of the cigarettes from the final stamp issue as described earlier but it does provide further evidence of the power of the postage stamp.

110 United States stamps: https://facts.usps.com/stamps accessed 9/7/2020. 


\section{Chapter 6. Postage Stamps Promoting Immunisation and Disease Control}

The promotion of universal immunisation cover has been a major stamp theme of many states over several years. While the thrust of immunisation is directed mainly to the health and welfare of children, by necessity, the messages are directed at their parents as part of an overall child health policy. In New Zealand, for example, immunisation is ranked as one of the top six health targets set by the New Zealand Government. ${ }^{111}$ Despite this, New Zealand Post has never issued a stamp supporting immunisation and especially in the long series of so-called 'health stamps'.112

A major triumph for the WHO vaccination policy was the elimination of variola from the world and this event has been recorded by stamp issues (figure 26 and 48). There have been several approaches to stamp design, and these have included propaganda against individual diseases, information about the range of conditions at which immunisation can be targeted and the techniques of immunisation. Figure 48 also includes examples of stamps that list common diseases for which suitable vaccines are available.

Many immunisation stamps have emanated from low-income countries where deaths from common infectious diseases are common. These states usually employ image designs predominantly, to address problems of literacy and also in those states were several languages are spoken. Overall, the images used are quite different from those in the antismoking campaigns and graphic images are rarely used. Conversely, the images are designed to raise the profile of immunisation and to encourage large scale participation and thus to develop herd immunity.

Poliomyelitis is an example of a disease that can have terrible consequences when acquired but can be successfully prevented by immunisation. The introduction of the first polio vaccine and the subsequent course of events associated with it have all been reflected in postage stamp images. Stamps have played many different roles. They have helped to raise public awareness about this crippling disease and the ready availability and the ease of administration of the vaccine. With the fall in prevalence of the infection, they have acted as

\footnotetext{
111 Health targets in New Zealand:

https://www.health.govt.nz/new-zealand-health-system/health-targets/about-health-targets/health-targetsincreased-immunisation accessed 13/7/2020.

112 The Len Jury New Zealand Stamp Catalogue, New Plymouth: Philatelic Distributors Limited, 2019.
} 
reminders to the population of the consequences of failing to immunise their children. This subsection reviews and illustrates the range of stamps that have been produced with the aim of education, health promotion and warning to society of the seriousness of this disease.
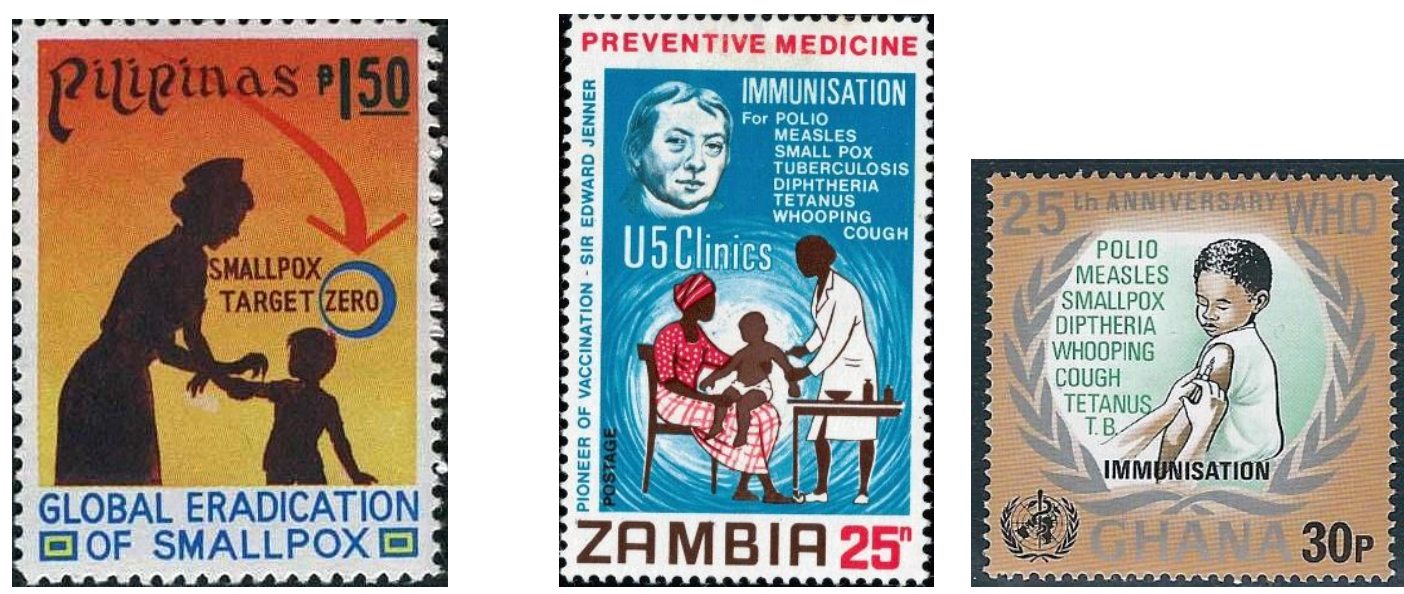

Towards smallpox eradication, Philippines, 1978, the range of immunisation available in the named country, Zambia, 1970, Ghana, 1973.

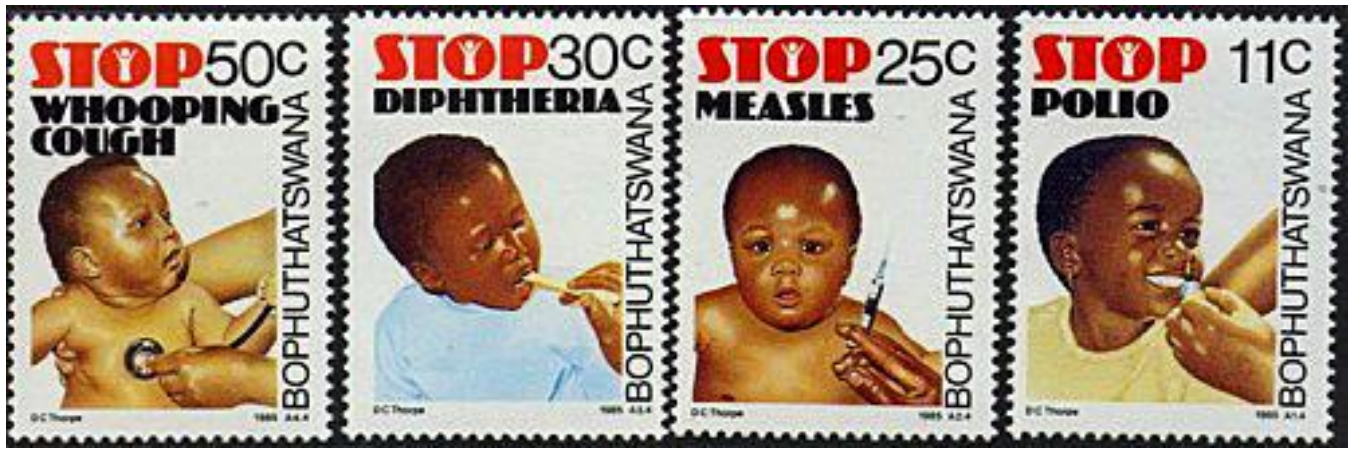

Figure 49. Immunisation designs encouraging immunisation against named diseases, South African territory of Bophuthatswana, 1985.

In an attempt to repeat the success of smallpox eradication, the WHO introduced the Global Polio Eradication Initiative to end poliomyelitis as one of the major diseases of mankind. ${ }^{113}$ The aim of total eradication has not yet been totally achieved but figure 49 indicates the success that has been achieved over thirty years. In 1988, there were 125 countries with endemic polio. Currently there are two countries with endemic polio, Afghanistan and Pakistan. While this state of affairs continues all other countries remain at risk of importing

\footnotetext{
${ }^{113}$ WHO polio campaign: https://www.who.int/countries/eth/areas/immunization/pei/en/ accessed $15 / 7 / 2020$
} 
polio and therefore require keeping high immunisation levels in place. Pakistan has used postage stamps to promote its campaign to be free of polio and other countries have used the postal medium also to promote polio eradication. The Pakistan issue is particularly important, as it shows women being associated with vaccination, as delays in the vaccination campaign have been caused by ethnic, gender and religious differences (figure 50). ${ }^{114}$

In earlier years, several countries produced stamps with images of children damaged by poliomyelitis. These stamps usually depicted children on crutches as a result of paralytic polio. Although none portrayed the very serious events of respiratory paralysis and death.
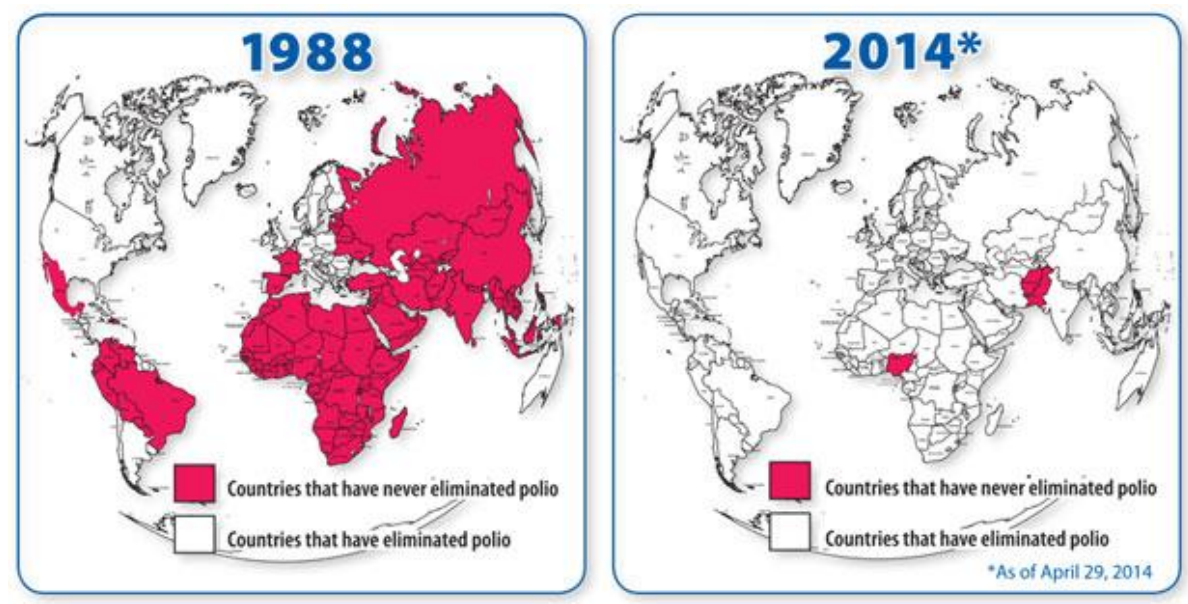

Results of poliomyelitis eradication programme from 1988 to 2014 in global terms. ${ }^{115}$
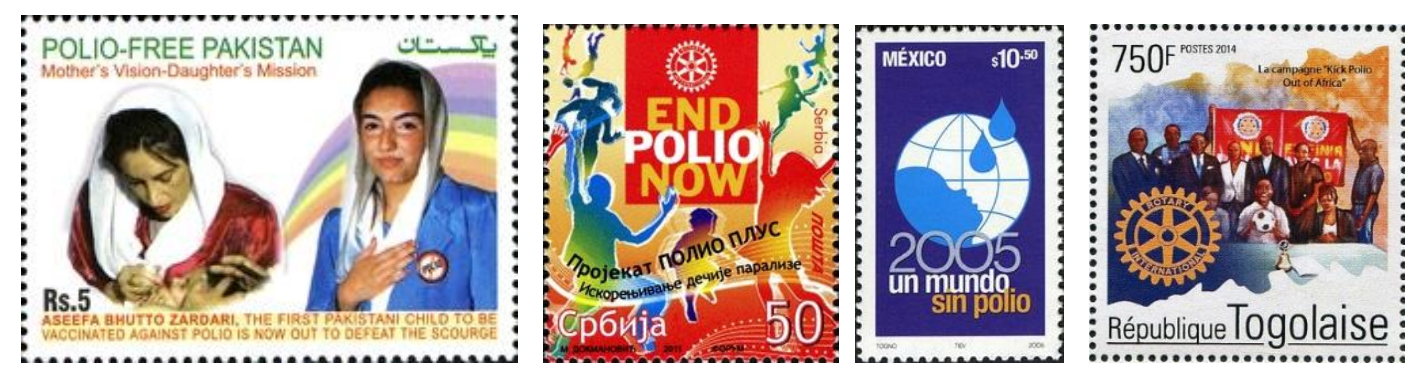

Figure 50. Polio-free Pakistan, 2009, “End polio now” Serbia, 2011, “A world without polio”, Mexico, 2005, "Kick polio out of Africa", Togo, 2014.

\footnotetext{
${ }_{114}$ Polio in Pakistan: https://www.aljazeera.com/indepth/features/pakistan-polio-comeback-vaccine-boycotts191226142838246.html accessed 21/7/2020.

115 Polio eradication results: https://www.immune.org.nz/sites/default/files/polio-map 1988-

2014 616x312px.jpg accessed 15/7/2020.
} 
One United States stamp did honour those who had helped fight polio (figure 51). A more recent Canadian stamp reminded society of these passed events and the hope that crutches associated with polio could be cast off for ever if high immunisation rates were maintained.
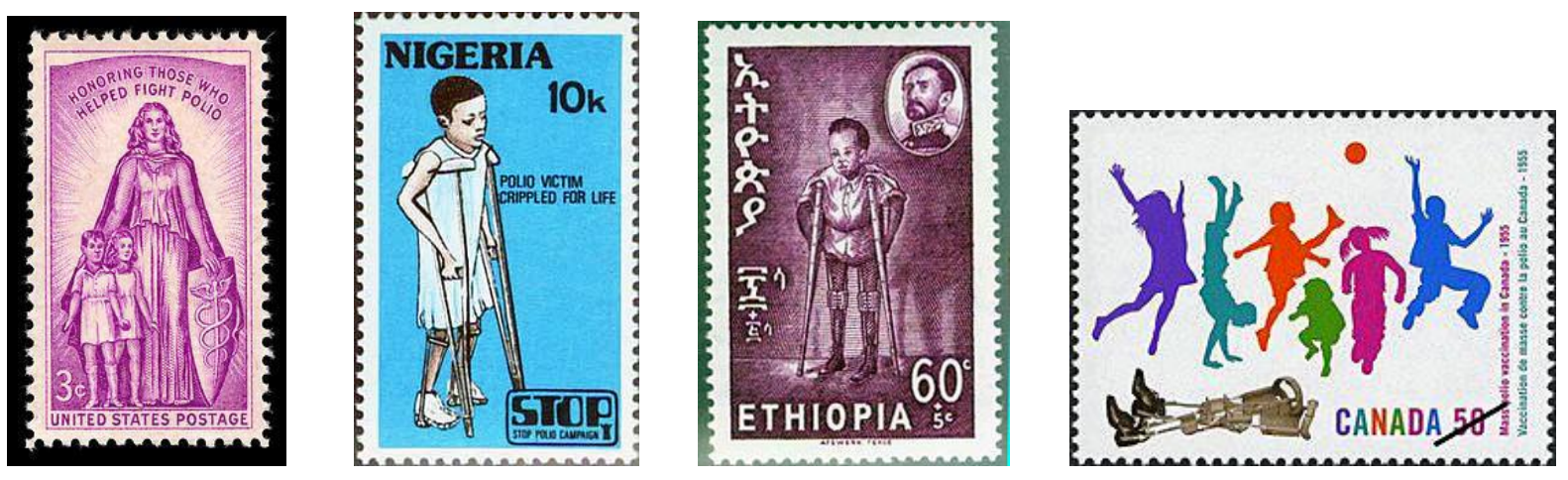

Figure 51. "Honouring those who helped fight polio", USA, 1957, the crippling effects of polio, Nigeria 1984, Ethiopia, 1963, mass vaccination against polio in Canada, 2005.

The development of an effective vaccine against poliomyelitis has been one of the major medical advances in the $20^{\text {th }}$ century. Jonas Salk developed the inactivated (killed)(IPV) polio vaccine in 1955 which has to be given by an intramuscular or intradermal injection. This was followed by an oral vaccine developed by Albert Sabin in the 1960s. This vaccine is now the principle vaccine used in the eradication programmes and as an attenuated vaccine it is less likely to enter the central nervous system. However, as polio is eradicated there is a return to the IPV format to prevent the rare vaccine associated paralytic poliomyelitis (VAPP). Human beings are the only reservoir of the polio virus and there is the potential to eliminate the disease completely and hence the importance of the WHO eradication programme. Postage stamps have played a valuable part in the history, information dissemination, education and promotion of polio vaccination.

While celebrating Jonas Salk's discovery the stamp images used have had an educational element also, in showing the site of injection and suggesting the ease of administration by including a picture of a relaxed child. Other stamps simply make a plea to vaccinate one's children. Figure 52 indicates a celebration of Salk, himself, the immunisation technique used and pleas for vaccination. The photograph shows the source image for two of the issues. With the widespread introduction and use of the oral vaccine, there has been an abundance of stamps issued encouraging the use of the vaccine. Many of these illustrate the ease of its use and stressing the few oral drops required to achieve the end result. Combined with 
these images, is often recognition of the work of Sabin in developing the vaccine. Figure 53 shows Albert Sabin, his administration of the vaccine to a child and a Peruvian stamp portraying the same action. A well-recognised stamp is the Indian issue of 1994 . The stamp image stresses the importance of the oral polio vaccine in helping India to eliminate poliomyelitis and the importance of continued immunisation (figure 54). This figure also shows a range of issues promoting oral polio immunisation and its method of delivery. The final figure in this chapter is intended to show the varying approaches to immunisation by a wide geographic spread of countries (figure 55).
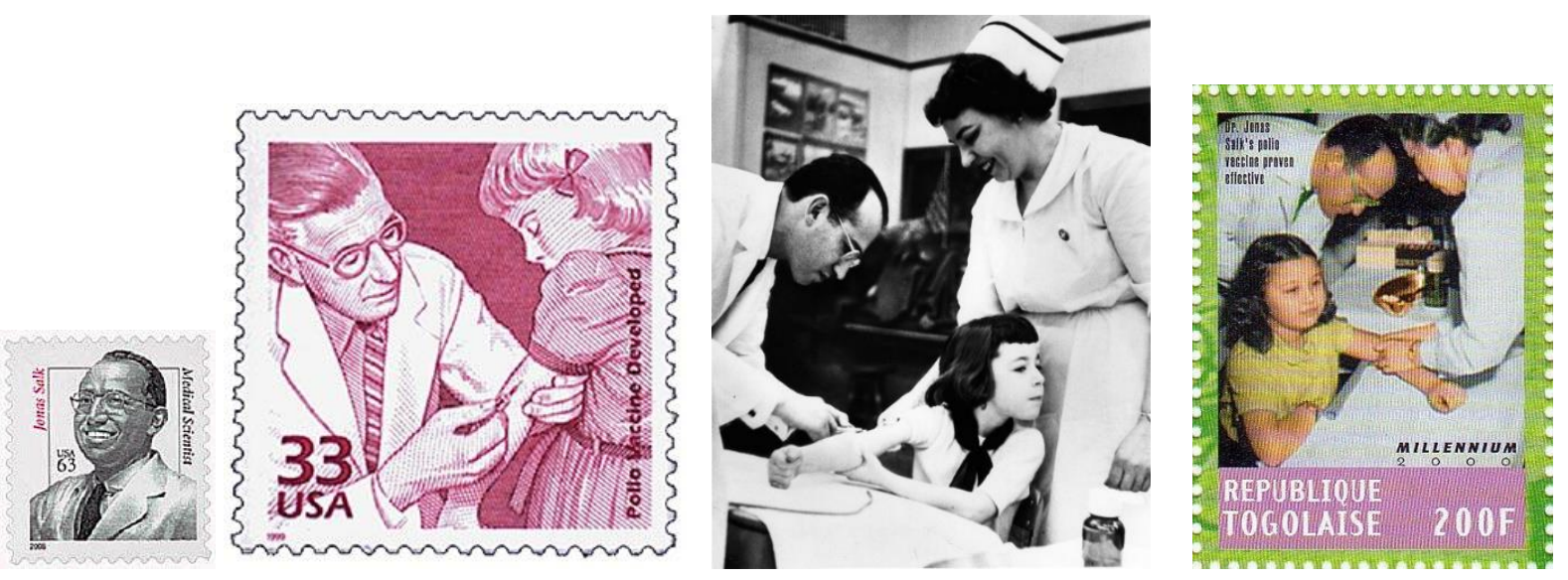

Jonas Salk, USA, 2000, Polio vaccine development, USA, 1999, Effective polio vaccine, Togo, 2000, Photograph: Jonas Salk and a nurse administering the vaccine. ${ }^{116}$
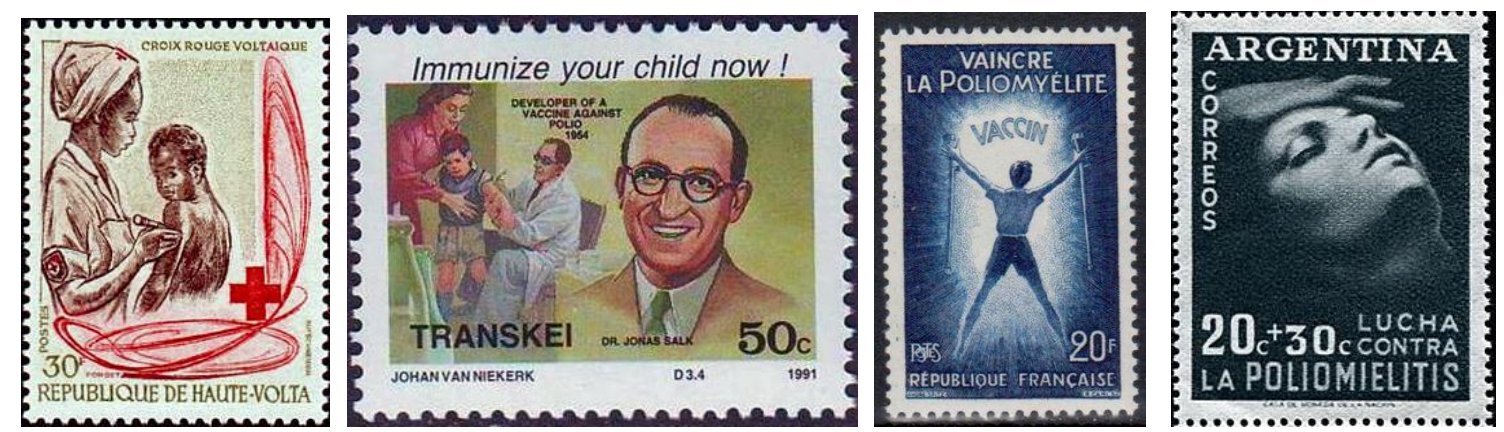

Figure 52. Immunisation site, Upper Volta, 1970, Jonas Salk and immunisation, Transkei, 1991, “Defeat poliomyelitis", France, 1959, campaign against polio, Argentina, 1956.

\footnotetext{
${ }^{116}$ Source: Jonas Salk Polio Vaccine Collection, 1953-2005, UA.90. F89, Archives Service Center, University of Pittsburgh, USA.
} 

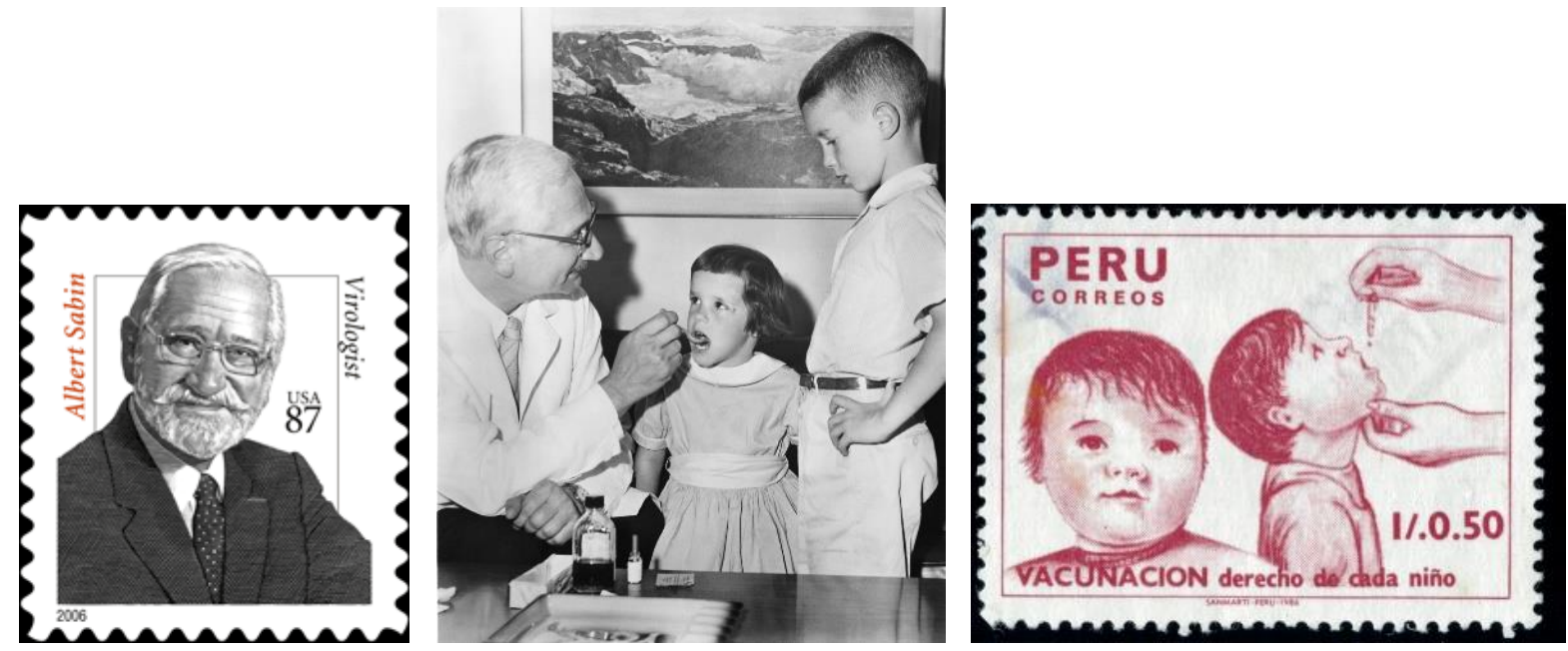

Figure 53. Albert Sabin, virologist, USA, 2006, photograph of Polio vaccine development

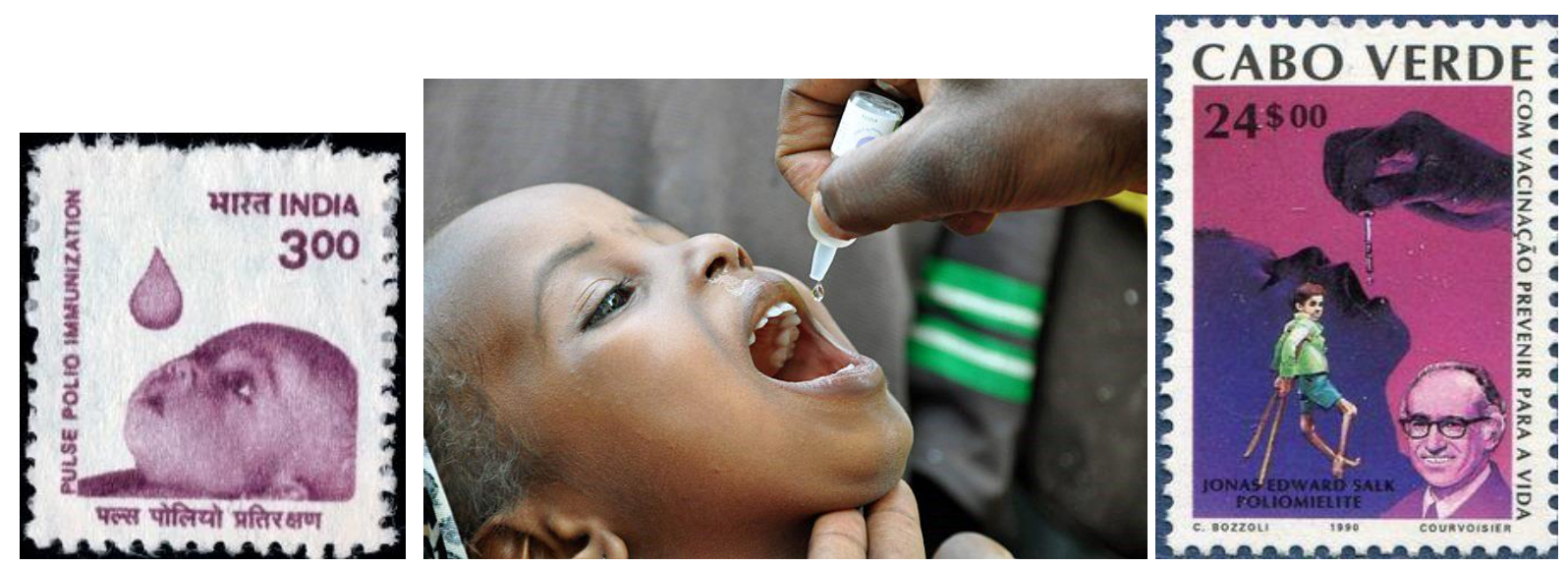

Figure 54. Oral polio vaccine, India, 1994, Photograph of child receiving oral drops, oral vaccine and a reminder of the consequences of failure, Cape Verde Islands, $1990 .{ }^{117}$
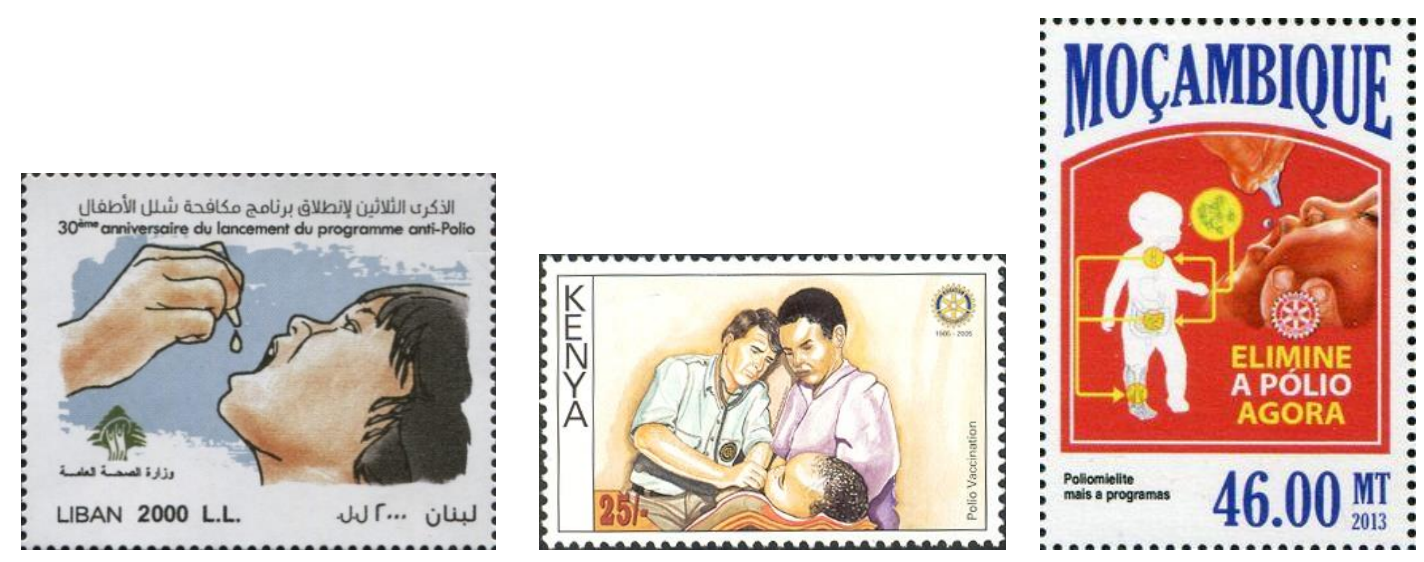

117 Photograph of oral polio drops:

http://polioeradication.org/wp-content/uploads/2016/07/13 Polio-imm-2-2.jpg accessed 31/7/2020 

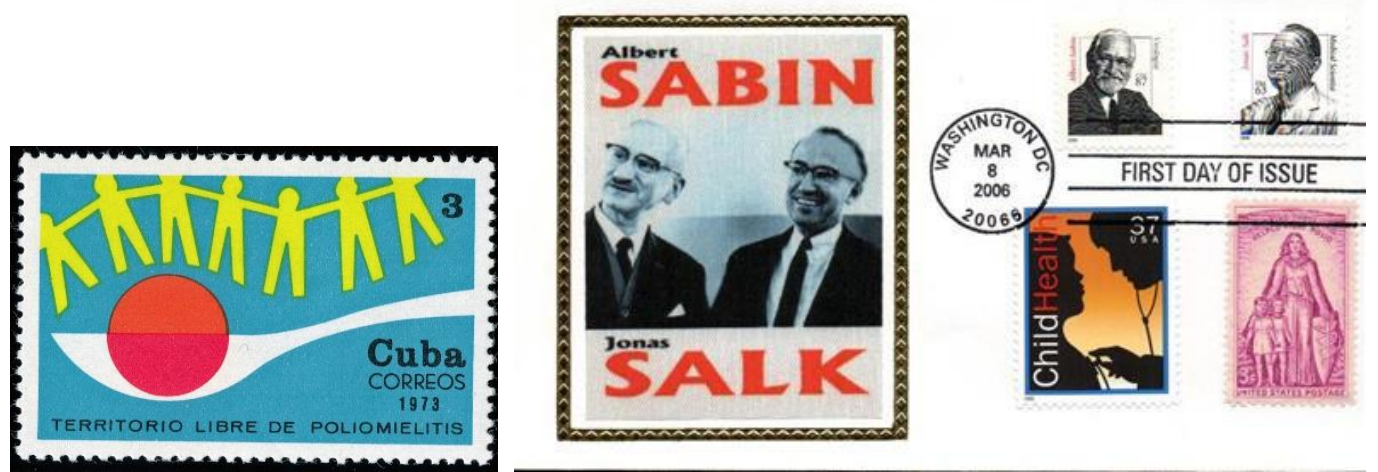

Figure 55. Oral polio immunisation, Lebanon, 2000, Kenya, 2005, Mocambique, 2013, Cuba, 1973. First Day Cover, celebrating both Sabin and Salk, USA, 2006.

Analysis of the data presented in this chapter strongly supports the argument that there has been a very wide international use of postage stamps to promote immunisation against infectious diseases. The intention, of course, is the prevention of the diseases that cause death and damage to humans and especially to children. Poliomyelitis has been selected as a specific infectious disease, and to look more closely and show how stamps have been used to promote immunisation and its prevention with the hope of the disease's eventual elimination. The ease of the delivery of the oral vaccine has led to its widespread use and this fact is well reflected in the many postage stamps that have been issued on the topic by many nations that promote visually the ease of delivery of the vaccine. Again, the use of stamps in such large numbers, over a long period, and from so many countries suggests that these stamps have been perceived as being valuable in the promotion of immunisation and health education. 


\section{Chapter 7. \\ Miniature Messages Promoting Nutrition, Growth and Development in Childhood.}

\subsection{Breast Feeding}

Human breast milk is the perfect food for the mature infant during the first six months of life and provides all the energy and nutrients needed. It also protects infants from respiratory and intestinal infections. ${ }^{118}$ However, nearly two out of three infants worldwide are not exclusively breast fed during the first six months of life. The inappropriate marketing of breast milk substitutes has undermined the efforts to improve breast feeding rates and the duration of breast feeding. ${ }^{119}$

To counter this trend many initiatives have been introduced such as baby-friendly hospitals, world breast feeding week, and legislation, in some countries, to prevent harmful marketing of substitutes. An international code of marketing breast milk substitutes has been introduced. Multi-media have been used to spread the message that breast feeding is important for children as it improves health through to adult life and results in lower mortality and morbidity and also reduces the risk of chronic diseases in later life. ${ }^{120}$ This section illustrates and discusses the contribution made by postage stamps in helping to impart the important messages about breast feeding. Many illustrations are included to indicate the wide use of the postage stamp and the many differing presentations upon the theme of encouraging breast feeding.

In many countries, including Western nations, there are still taboos around breast feeding in public. ${ }^{121}$ Stamps illustrating actual breast feeding can therefore clash with the cultural norms of certain countries. A good example of this is in India where the exposure of women's breasts raises cultural barriers. Pratiga Baskota, in 2018, explored this subject and wrote:

\footnotetext{
118 Ministry of Health, New Zealand: https://www.health.govt.nz/your-health/pregnancy-and-kids/firstyear/helpful-advice-during-first-year/breastfeeding-perfect-you-and-your-baby accessed 8/8/2020

119 Breast feeding rates: https://www.who.int/health-topics/breastfeeding\#tab=tab 1 accessed 8/8/2020.

120 Clive R. Belfield, Inas Rashad Kelly, "The benefits of breast feeding across the early years of childhood", The Journal of Human Capital, 2012; 6: 251-277.

${ }^{121}$ Zeljka Buturovic, Suzana Ignjatovic, "Attitudes towards breastfeeding and breastfeeding practice: lack of support for breastfeeding in public as a factor in low breastfeeding rates", Journal of Applied Health Science, 2017; 3: 137-143.
} 
The cover picture of the Grihalakshmi magazine recently ignited a huge debate throughout the country about the portrayal of a woman breastfeeding openly. The model, Gilu Joseph, and the fortnightly faced a lot of criticism. But what many don't know is that in the 1980s (sic), our very own government released a postal stamp that featured a mother breastfeeding a child - without covering up. ${ }^{122}$

This contentious stamp is illustrated in figure 56. It was one of a large series of seventeen Indian stamps portraying aspects of Indian life.
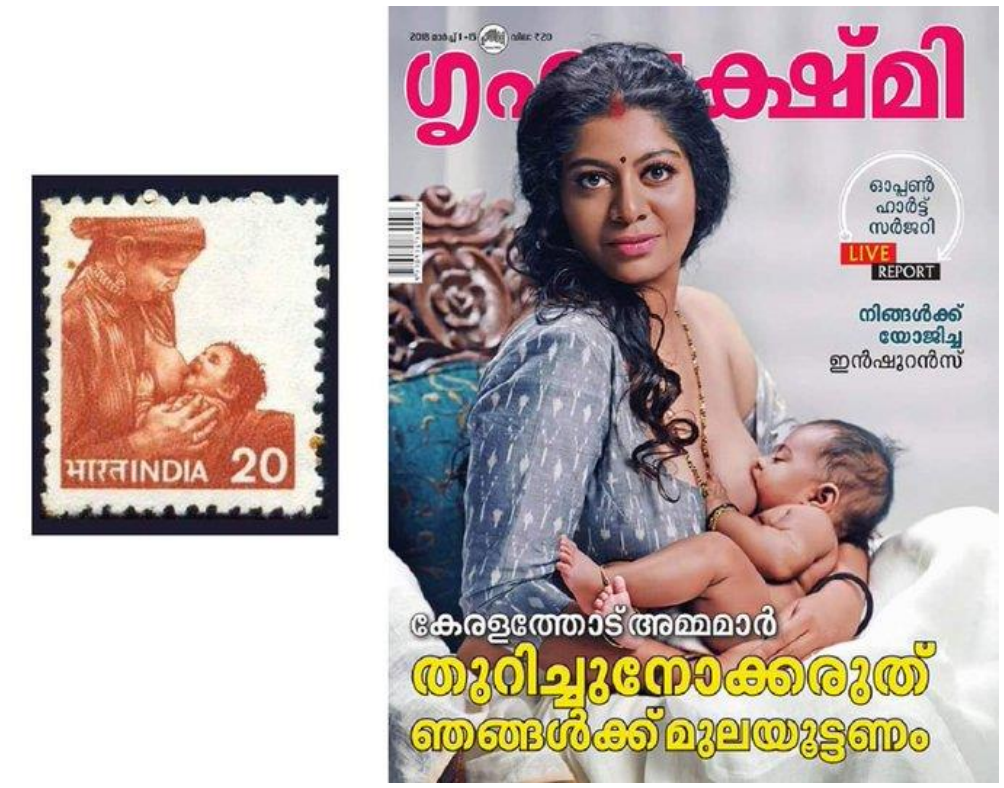

Figure 56. Woman breast feeding, India, 1981, Indian magazine portraying breast feeding. ${ }^{123}$

While encouraging breast feeding certain postal issues have portrayed the message that breast feeding is the norm and bottle feeding is bad. Figure 57 shows two good examples of the negative approach to bottle feeding. Stamp designs on the topic of breast feeding therefore, do vary from the more conservative countries, to those with more relaxed attitudes to breast feeding as in central and southern Africa. Figure 58 illustrates this point by presenting examples of stamps produced by various regions of the world and they demonstrate the differing approaches to delivering the message that breast feeding is normal and preferable.

\footnotetext{
122 Pratigya Baskota, The New Indian Express, $8^{\text {th }}$ March 2018. The stamp was actually issued on 25/3/1981.

${ }^{123}$ Magazine: https://www.indiatimes.com/news/india/all-those-complaining-about-grihalakshmicover-must-see-this-indian-postal-stamp-from-1984 accessed 18/8/2020.
} 

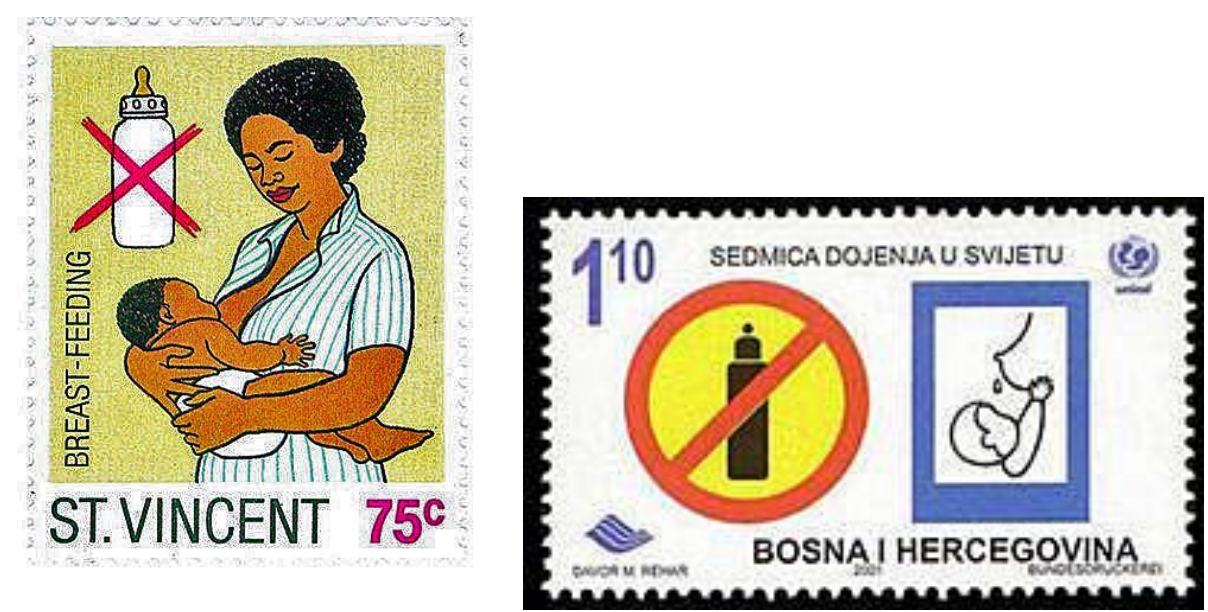

Figure 57.

"Breast is best", St. Vincent, 1987, Bosnia, 2001.

A sample of stamps showing the range of design that has been used to promote the subject of breast feeding is shown in figure 58. It also reveals some cultural differences in their presentation and overall, it confirms the world wide utilisation of breast feeding stamps. However, as with anti-smoking stamps, the United Kingdom and the United States are at odds with most other countries by choosing not to promote breast feeding by stamp issues. One can only hypothesise that this may be related again to their production of substitute milk formulae. The United States has not adopted the international code of marketing breast milk substitutes.

In relation to the debate that arose in India over the revealing of women's breasts while feeding (figure 56), no such cultural barriers appear to exist in the European stamps presented. Similarly, African and Pacific Island nations appear to have no reservations in presenting active breast feeding on their postage stamps. A more reserved approach is seen in the Islamic states of Algeria, Iran and Bangladesh, but the importance of breast feeding is still promoted and displayed. Overall, the stamps produced by all countries promote the view that breast feeding is the natural way of feeding and is a normal activity, the latter being well shown in the stamp of Honduras with a policewoman feeding while on duty (figure 58).

Figure 58.

Europe 

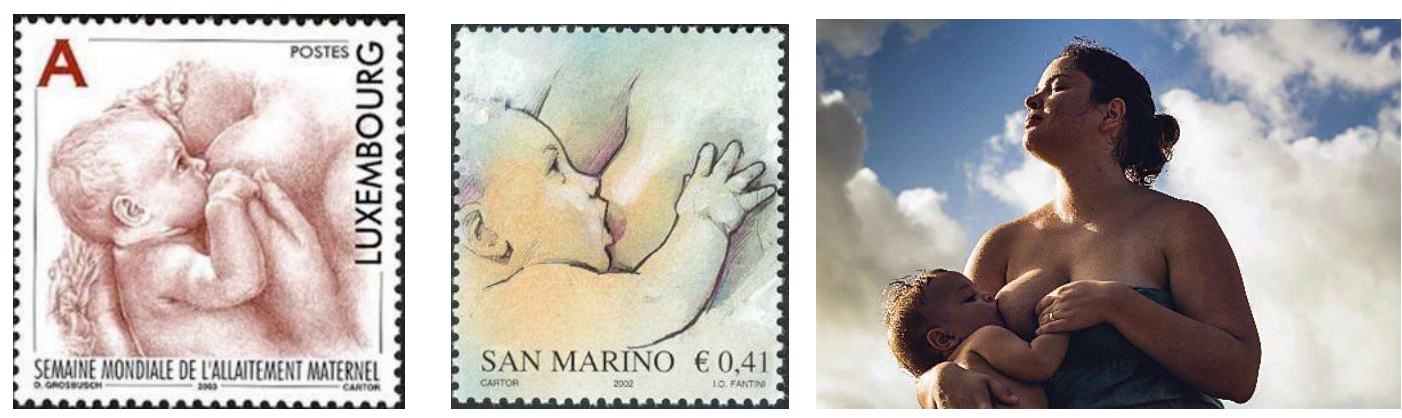

Breast feeding week, Luxembourg, 2003, San Marino, 2002, Photograph Photo credit: Flávio Correia Lima

East, Central and West Africa
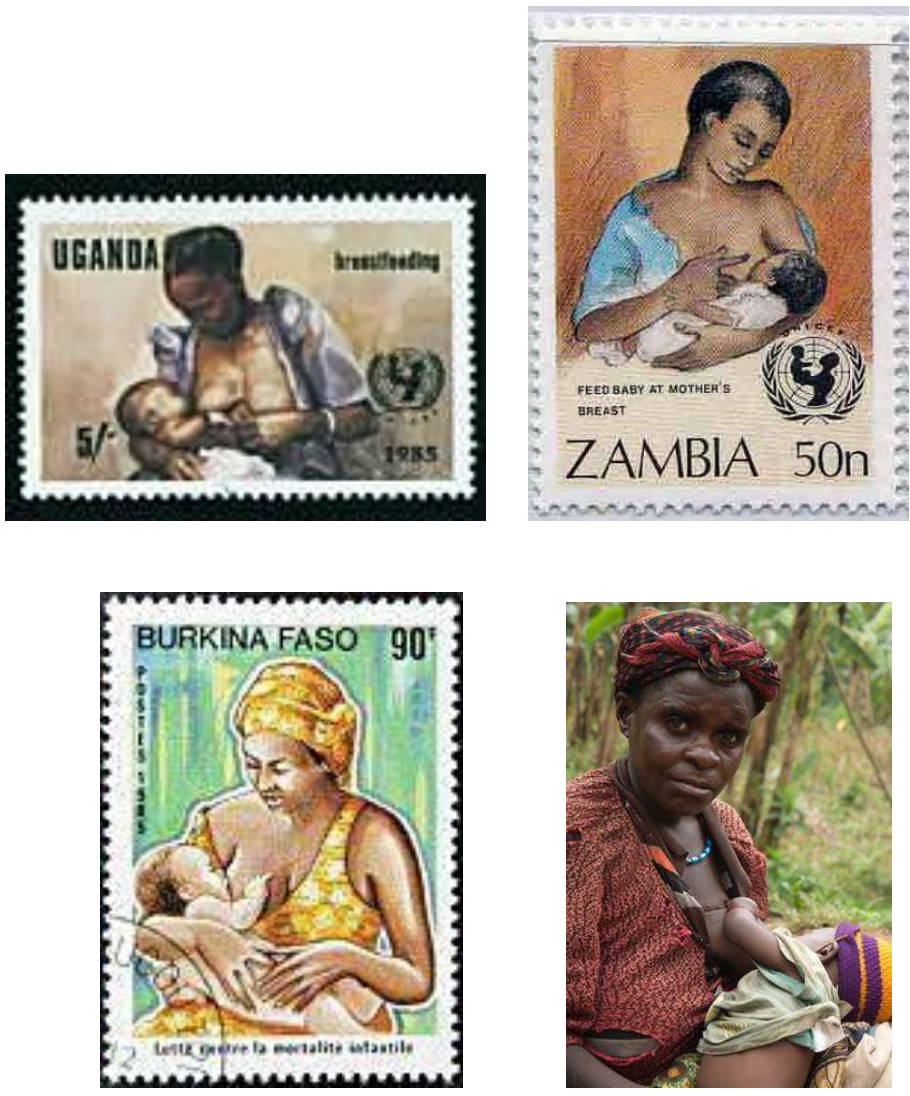

Breast feeding, Uganda, 1985, "Feed baby at mother's breast", Zambia, 1988, "Reduce infant mortality", Burkina Fasso, 1986, breast feeding in Africa. ${ }^{124}$

${ }^{124}$ Breast feeding in Africa, http://www.flickr.com accessed 18/8/2020. 

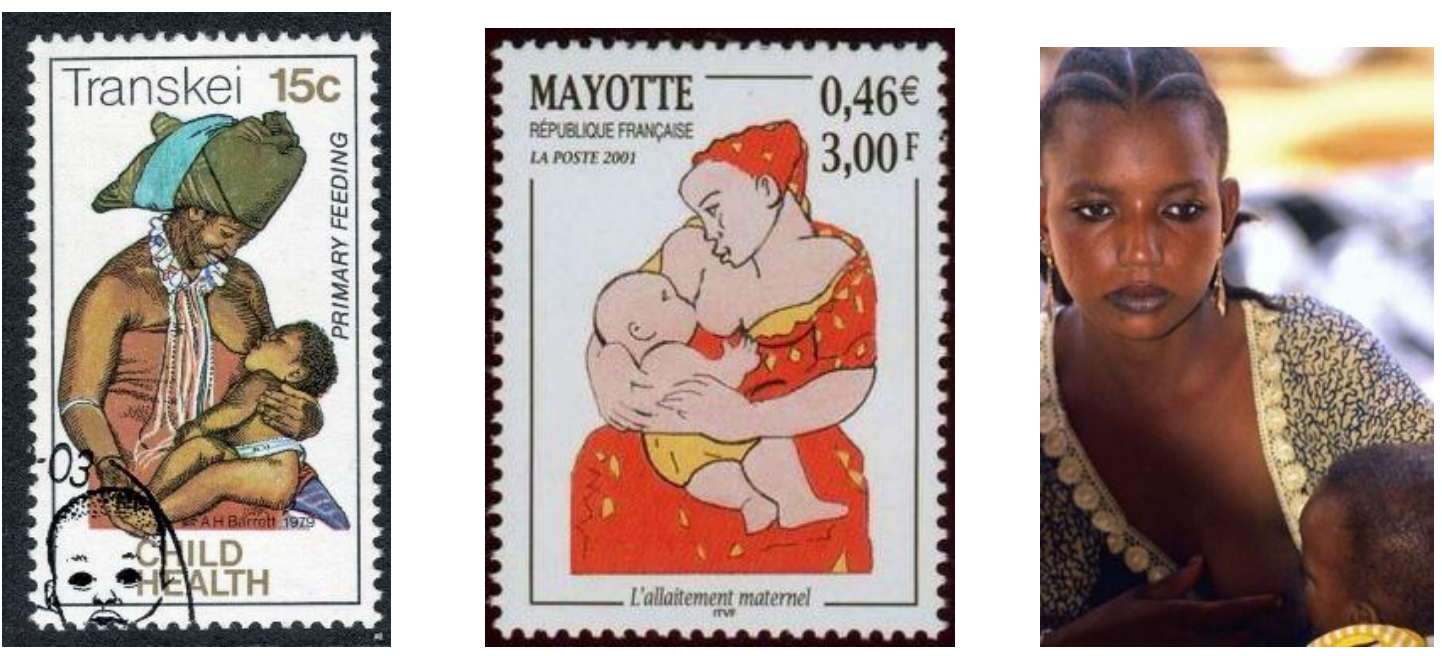

Primary feeding, Transkei, 1979, Maternal feeding, Mayotte (2001), photograph of breast feeding in Africa. ${ }^{125}$

North East and West Africa
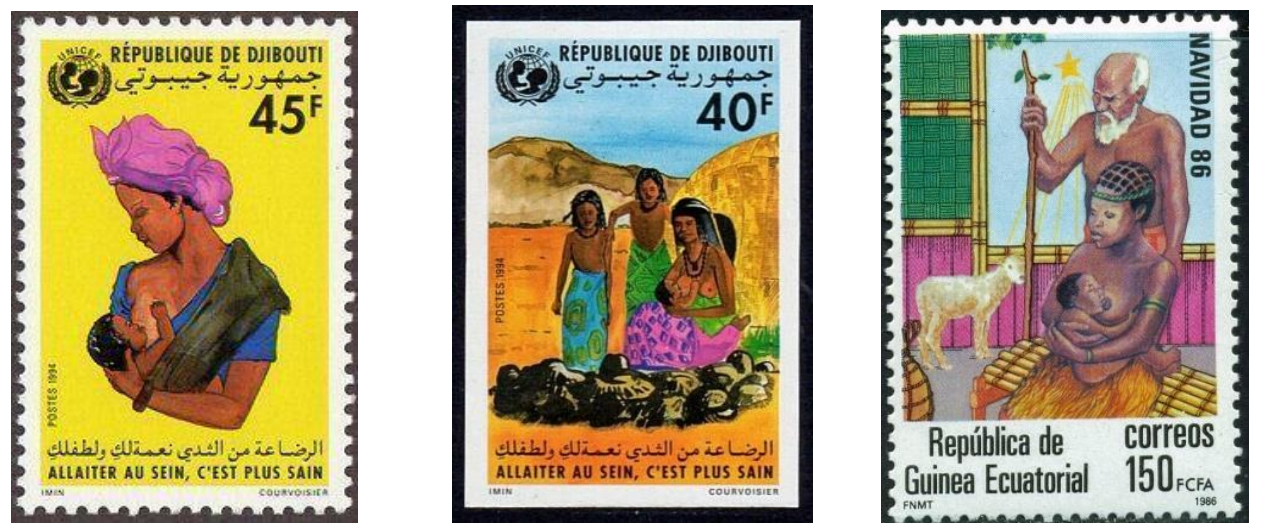

"Breast feeding is healthier", Djibouti, 1994, nativity scene with breast feeding, Equatorial Guinea, 1986.

\footnotetext{
${ }^{125}$ Breast feeding in Africa (2) http://www.beauty-of-africa.tumblr.com accessed 18/8/2020.
} 


\section{Oceania}
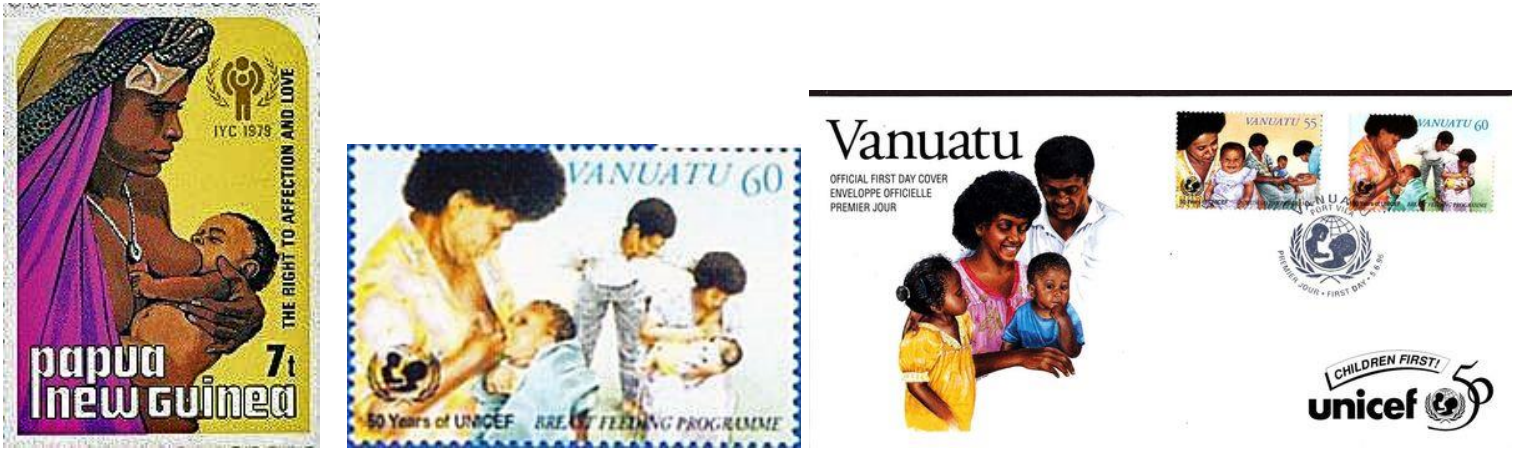

"The right to affection and love", Papua New Guinea, 1979, Breast feeding programme, Vanuatu and First Day Cover, 1996.

Islamic States
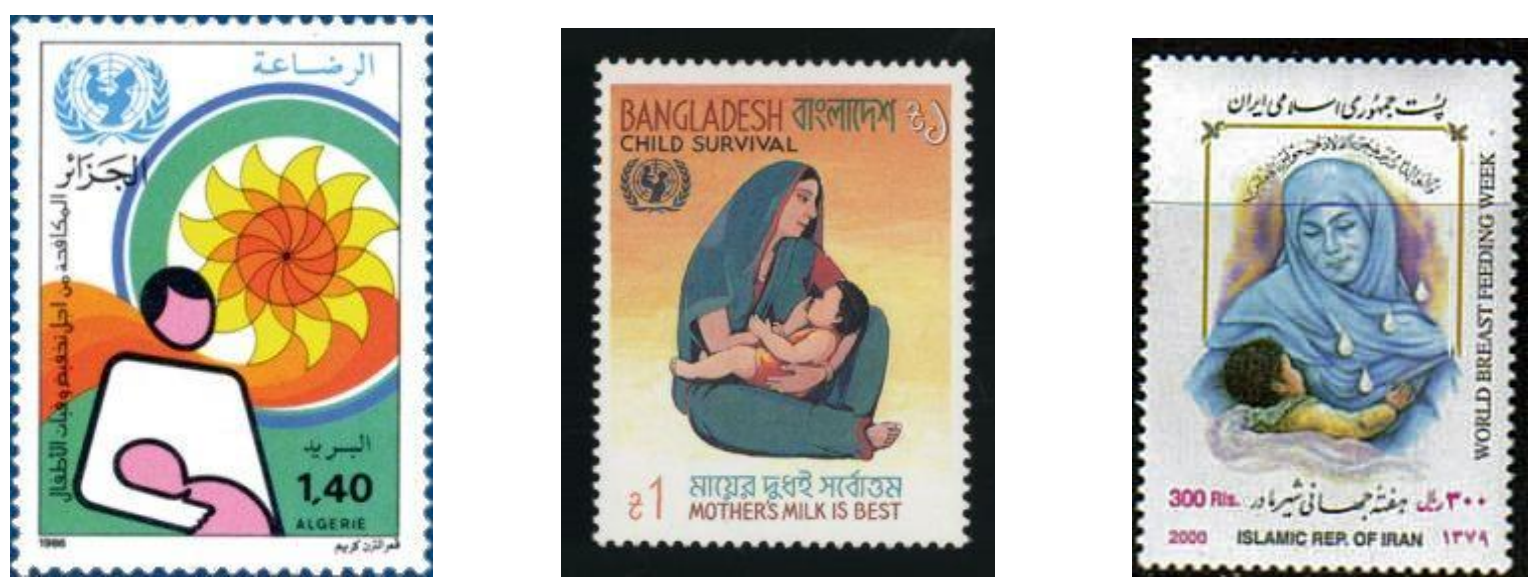

Breast feeding, Algeria, 1996, "Mother's milk is best", Bangladesh, 1996, "World breastfeeding week", Iran, 2000.

The Americas - Central America
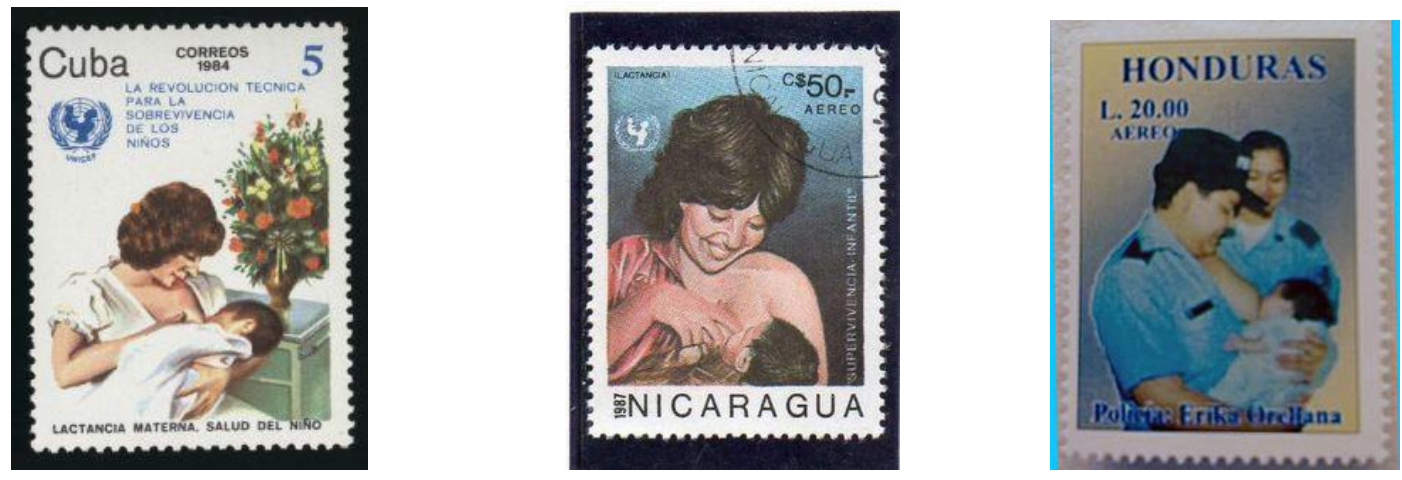

Figure 58. Infant survival campaigns, Cuba, 1984, and Nicaragua, 1987, Mother's Day, Honduras, 1999. 


\subsection{Child nutrition and growth}

While it is now recognised that in some low-and middle-income countries there coexists both overnutrition (overweight and obesity) along with undernutrition (stunting and wasting), in this section the concentration is upon undernutrition and growth in children. ${ }^{126}$ Among the world's children in 2020, 52 million under-fives are suffering from malnutrition as defined by a low weight for height ratio, which ultimately leads to stunting. In addition, it has been claimed that malnutrition is the main cause of death and disease in the world (see figure 59). ${ }^{127}$ This malnutrition is occurring despite the world now producing more food than ever before.

Early detection of growth failure requires frequent child surveillance and monitoring through child health clinics and the regular use of ethnically appropriate growth charts. Postage stamps have been used to help highlight the need for such clinics and to educate the population of various countries as to their importance. The stamps have also demonstrated the appearance of growth charts and promoted them as essential items in childcare. Figures 23 and 25 are examples of postage stamps that do exactly this and demonstrate the ideal weight and height gains. The third stamp in this group, from Dahomey, as early as 1942, shows the introduction of weighing at an infant clinic. Figure 25(O) indicates neurological testing at a child clinic. The child health clinic features on many

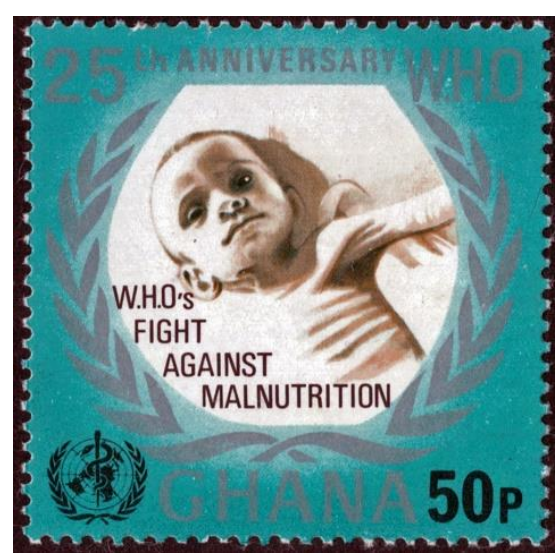

Figure 59.

“Fight against malnutrition”, Ghana, 1973.

126 Editorial, "The double burden of malnutrition", The Lancet, 2019; 395: 5.

127 Malnutrition: https://www.who.int/nutrition/topics/world-food-day-2019-malnutrition-world-healthcrisis/en/ accessed 20/8/2020 
many stamps from Africa and Oceania. These clinics are designed to provide the early screening and assessment tools for the detection of children at risk of malnutrition and growth delay. Figure 60 shows a range of designs that have been issued to encourage and normalise attendance at infant and child clinics.

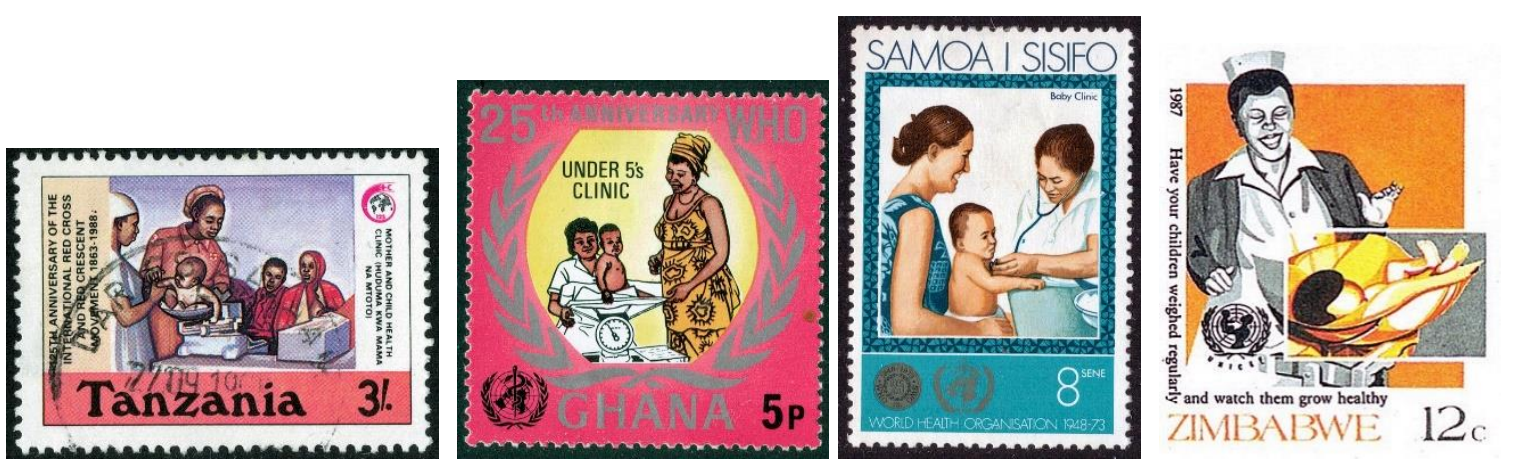

Mother and child health clinic, Tanzania, 1988, Under 5s clinic, Ghana, 1973, Samoa, 1973, Weight assessment, Zimbabwe, 1987.
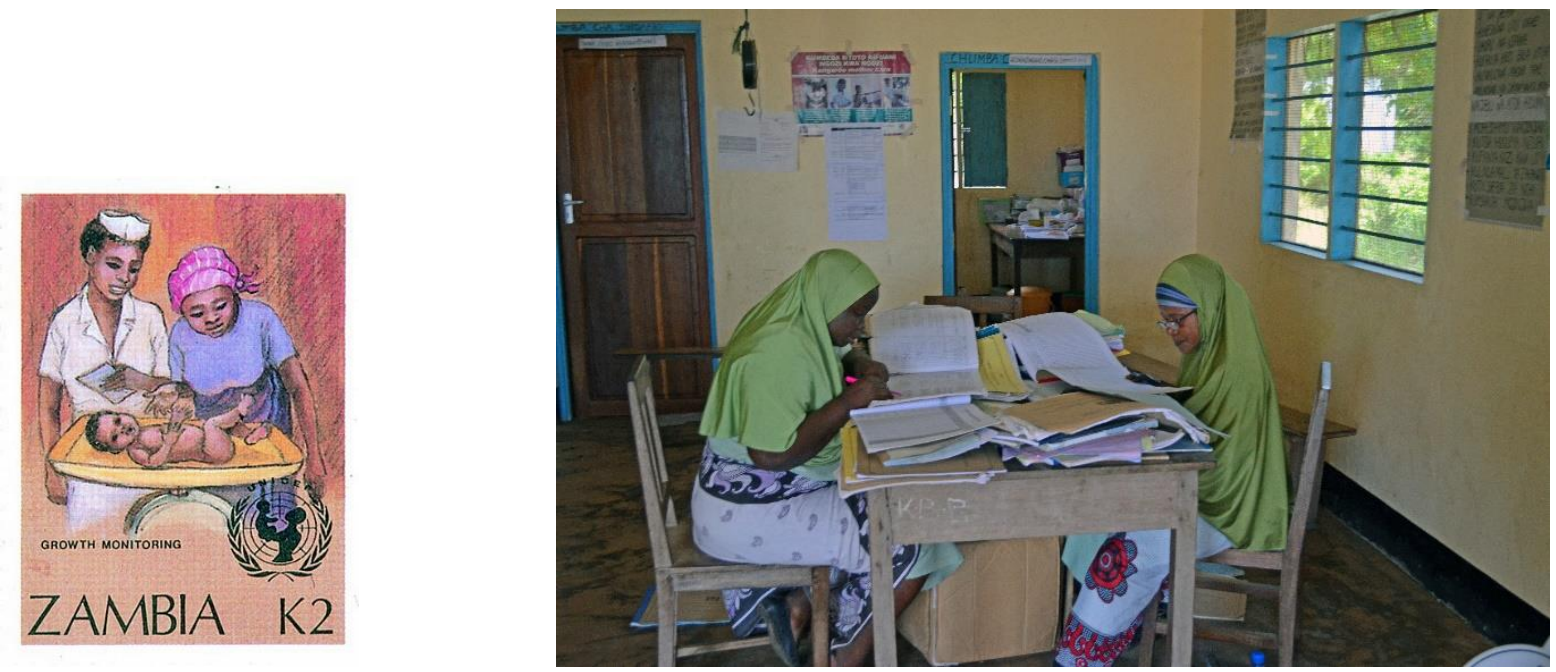

Figure 60. Growth monitoring, Zambia, 1988, Child clinic, Kilwa, Tanzania, photograph by the author.

Many other stamps have been produced to encourage better diets for young children as well as prenatal nutrition for expectant mothers and the need for clean water. One of the major improvements in health care for these children at risk has been the introduction of oral rehydration therapy in the treatment of diarrhoeal illness. Examples of these stamps are demonstrated in figure 61. 

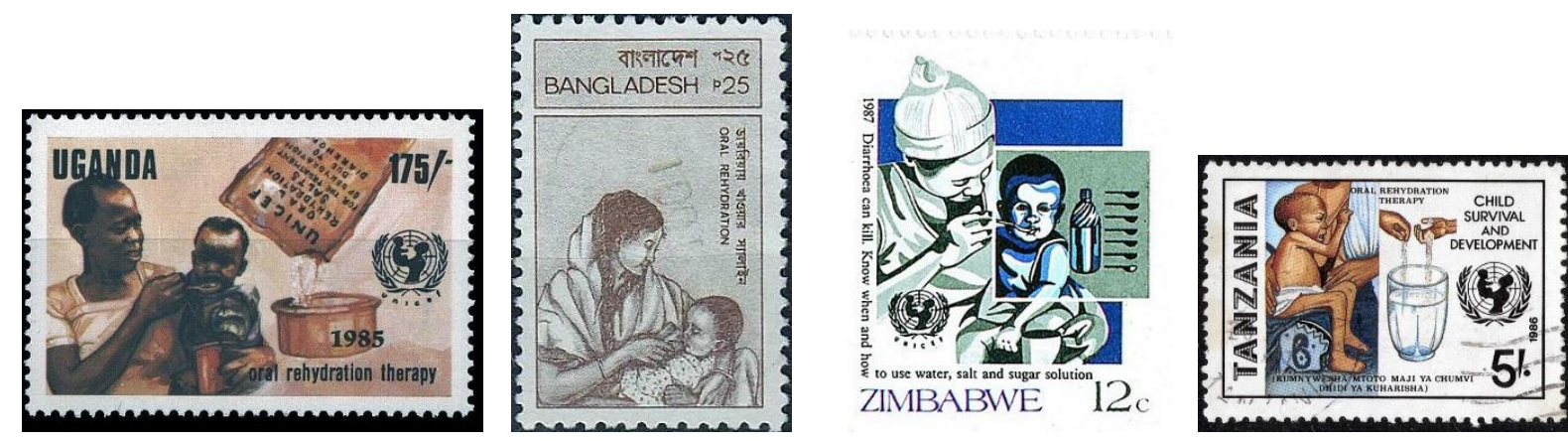

Oral rehydration therapy, Uganda, 1985, Bangladesh, 1988, Zimbabwe, 1987, Tanzania,1986.
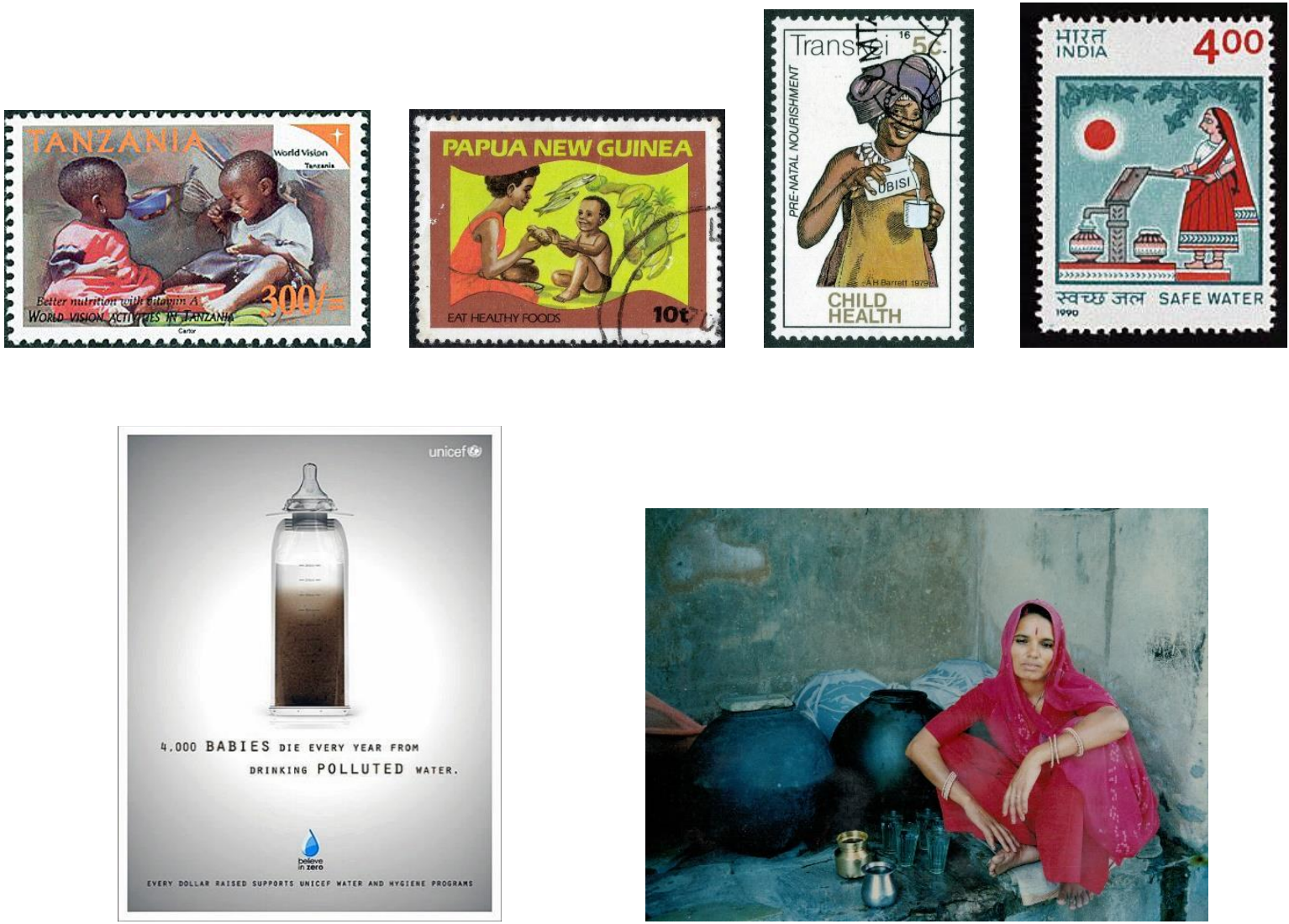

Figure 61. "Better nutrition”, Tanzania, 2005, Healthy eating, Papua New Guinea, 1982, Pre-natal nourishment, Transkei, 1979, "Safe water", India, 1990, UNICEF advertisement for safe water: source UNICEF, Lady selling water, India, photograph by author.

This chapter has focussed upon child nutrition and the importance of breast feeding and how stamps have been utilised to promote the practice. This is yet another health field where postage stamps have been employed to educate and promote good health care. Malnutrition and failure to grow seem to predominate in the low-income tropical countries of Africa and it is not surprising that the bulk of stamps have emanated from African countries. It is clear that stamp information can only play a tiny part in addressing the enormous and serious problem of malnutrition and failure to thrive. The stamp's role, 
however, may be important in conveying to the population that attendance at health clinics is a normal and vital way of screening children for growth failure and it is a normal part of child rearing even in the most resource poor countries. This view is supported by the promotion of these stamps in leading medical journals such as Archives of Disease in Childhood. ${ }^{128}$ Other stamps have stressed the importance of a balanced diet when food is freely available.

One of the compounding factors related to failure to thrive is the high incidence of gastroenteritis in tropical countries leading to fluid and electrolyte loss and hence weight loss. Several countries have released stamps drawing attention to the treatment of diarrhoeal illness and fluid loss by electrolyte solutions and the way in which they can be constituted. These solutions have saved many lives in Africa and elsewhere and the stamps may have had an important educational role in this situation (see figure 61).

128 Mike Davis, A.J. Mayne, "Stamps in paediatrics", Archives of Disease in Childhood, 2000; 82: 20. John Pearn, "Paediatrica philatelica", Journal of Paediatrics and Child Health, 1999; 35: 232-236. Kenneth Dawson, "Paediatric philately: gastroenteritis and rehydration", Middle East Paediatrics, 2004; 9: 29. 


\section{Chapter 8. Health Promotion and Postage Stamps: Changing Concepts Over Time.}

The first postage stamps to support a health project were issued by New South Wales in 1897. These were semi-postal stamps raising funds to help those with tuberculosis (see figure 10). Stamps have continued to be issued with health education and promotion as their primary theme from that date through to 2020 (see figures 15 and 26). During this period of 123 years, not only the methods of delivery of health information have changed but the whole concept of what constitutes health promotion and education have altered. This chapter is concerned with these changes, not only in what constitutes health promotion but in the adaptation of stamps to these altered viewpoints. It should be reiterated at this point, that after the initial stamps of New South Wales, the discussion is not concerned, in the main, with money-raising charity stamps unless they contain a health message.

In the Introduction to this work, health promotion was defined along the lines of the Ottawa Charter for Health Promotion, which states that health promotion was the process of enabling people to increase control over, and improve, their health. ${ }^{9}$ This was construed as meaning that health was a resource for everyday living. This definition has been challenged by the American Journal of Health Promotion on several points. ${ }^{129}$ They argued that "Health is an optimal state with physical, social, mental and spiritual aspects and that the role of health promotion is to change lifestyle and support people to do this". The counter argument states that health is a co-existent state and well-being is the key factor, especially in those with a disability. This in turn, raises questions of how one evaluates such a broad concept and how scientific rigour can be brought to bear. The solutions to this dilemma are now well described in the literature. ${ }^{130}$

To add to the complex picture, debate exists between disease prevention and health promotion. According to Maurice Mittelmark et al., disease prevention is mostly to do with reducing the burden of chronic and infectious disease by prevention and reducing risk factors. ${ }^{131}$ They argue that health promotion, while concerned with the above, places more

\footnotetext{
${ }^{129}$ M. O'Donnell, “Definition of health promotion”, Part 3, American Journal of Health Promotion, $1989 ; 3: 5$.

130 Don Nutbeam, "Evaluating health promotion - progress, problems and solutions", Health Promotion International, 1998; 13: 27-44.

${ }^{131}$ Maurice Mittelmark et al., "Health Promotion” 2017: 451. Ibid.
} 
emphasis on building communities, capacity to identify and solve health issues. Health promotion and disease prevention are complementary approaches to improving public health. There is further emphasis on empowering people to control their own health and building the community's capacity to solve health issues. ${ }^{132}$ In reality it is difficult to have a universally acceptable definition of health promotion that is specific and encompasses the several viewpoints of a number of professional groups.

These differing views have resulted in several models of health promotion becoming apparent. One represents the so called "medical" model which is technological in nature as compared to the more "social" models which take into account the causes of disease resulting from social and environmental factors and stresses the empowerment of the individual which it regards as central to health promotion. The medical model was strongly criticised by Ivan Illich as being harmful rather than being productive and was doctor centred rather than patient centred. ${ }^{133}$

If empowerment is central to promoting health (primacy of empowerment), how can that be achieved? Mittelmark et al. have outlined several models - health action model, the community empowerment model, social interaction skills, development of coalitions and education and social action models. A final model listed by them is that of media advocacy. ${ }^{134}$ All these different models are designed to achieve the goal of patient and community empowerment. There is a rapidly expanding literature on media advocacy, which has been defined as "the strategic use of mass media to advance public policy initiatives". ${ }^{135}$ What is important here is that traditional media try to fill the "knowledge gap", media advocacy addresses the "power gap". 136

If individual empowerment is key to improving health, how can people make better choices and change their behaviour and hence wellbeing? One method that has gained in status is the so-called "nudge". ${ }^{137}$ Signild Vallgarda has described "nudge" as a method of creating

\footnotetext{
132 Don Mittelmark, Ilona Kickbusch, Irving Rootman, Angela Scriven, Keith Tones, Health promotion, Elsevier Inc. International Encyclopedia of Public Health, volume 3, 2017.

133 Pearce Wright, "Ivan Illich", The Lancet, 2003; 361: 185.

134 Mittelmark et al. "Health Promotion": 456-459.

135 Lawrence Wallack, "Media advocacy: a strategy for empowering people and communities", Journal of Public Health Policy, 1994; 15: 420-436.

${ }^{136}$ Lawrence Wallack, Lori Dorfman, "Media advocacy: a strategy for advancing policy and promoting health", Health Education Quarterly, 1996; 23: 293-317.

${ }^{137}$ R.S. Thaler, R. Cass, Nudge: improving decisions about health, wealth, and happiness, London: Penguin Books, 2008.
} 
health-enabling environments and reversing the trend to make less healthy choices. Individuals can helped by "choice architects" who will make people's lives "easier and longer". ${ }^{138}$ It would appear to the writer that this is a new form of paternalism, now removed from medical practice and along the lines of Illich's criticism, but now espoused by others as health promotion professionals. However, nudge advocates would claim that this new paternalism is under pinned by empirical research in psychology and economics. ${ }^{139}$ However, there is continued debate in the literature on this point.

Among the models of health promotion listed above, that which was labelled "media advocacy", needs further discussion. Mittelmark et al. state that this model employs media locally to achieve radical outcomes by targeting unhealthy situations in specific communities and seeks to influence key decision makers. ${ }^{140} \mathrm{It}$ is proposed here that certain stamp issues do fit into the category of health advocacy and some can be seen as social marketing. While it is clear that their effect will not produce radical outcomes, their main attribute is their ability to spread the message widely and hence add to the overall media effect. While stamp use for postal services has declined in the West, low income countries use postage stamps widely and this is, of course, where major health disparity exists and infections such as malaria, still cause major morbidity. With media advocacy emphasising institutional accountability, the stamps used to counter the tobacco industry and the artificial infant formulae companies fit well into the model. This proposal is in keeping with the concepts of Yehiel Limor and Ilan Tamir who have propounded the view that the use of postage stamps be defined as mass media. They argue further that postage stamps constitute a neglected area in media research and that there is rich potential in this research area. ${ }^{141}$ There is, however, already a body of published work on the role of postage stamps and aspects of health. These studies are in keeping with the concept that postage stamp information has developed and changed with time. The work of Kearns et al. has stressed the ability of stamps to promote health awareness and that of new medical life-saving procedures. ${ }^{142}$

\footnotetext{
138 Signild Vallgarda, “Nudge - A new and better way to improve health?" Health Policy, 2012; 104: 200-203.

$139 \mathrm{~J}-\mathrm{F}$. Ménard, "A 'Nudge' for public health ethics: libertarian paternalism as a framework for ethical analysis of public health interventions?" Public Health Ethics, 2010; 3: 229-238.

${ }^{140}$ L. Wallack, L. Dorfman, D. Jernigan, M. Themba, Media advocacy and public health: power for prevention. Newbury Park, California: Sage, 1993.

${ }^{141}$ Yehiel Limor, Ilan Tamir, "The neglected medium: postage stamps as mass media" Communication Theory, 2020; March: 1-15.

142 Robin Kearns, Tara M. Coleman, Jonathan Edmeades, “New Zealand children's health stamps: ideological artefacts linking health and place", Social Science and Medicine, 2019; 227: 19.
} 
Pramanik et al., have recently confirmed the usefulness of postage stamps as a health promotion tool in Nepal. They concluded that the combination of pictures with text on Nepalese stamps was an effective method of informing the general public of health problems and their prevention. ${ }^{143}$ These findings further support the value of postage stamps in health education in low income countries. Other authors have pointed out that despite the change generally to electronic communication, postage stamps still remain an important method of communication with regard to transfusion medicine and that stamps give a clear, strong and unique message. ${ }^{144}$ Similar claims have been made by authors in parallel medical specialties and have claimed that postage stamps have remained one of the best ways of distributing health messages through disparate populations. ${ }^{145}$ Limor and Tamir state that the public expect the information provided in mass media be current, reliable and accurate. In addition, the topics so presented are an interpretative act as is the manner in which they are presented. The public, thus, expect this information to be accurate and this is particularly important in health-related matters.

In terms of health promotion, Harlan J. Strauss studied the role of postage stamps in delivering their message by use of psychological concepts. He argued that stamps are a particularly efficient form of psychological propaganda because they are in a single medium, being visual in form. It is the reinforcement of the message each time the stamp is viewed and encountered, that is the basis of their success. He based his views on empirical work which consisted of twenty-seven working hypotheses which he tested. ${ }^{146}$ There has been further research work in establishing an educational value in the use of postage stamps in school education. ${ }^{147}$ There does seem sufficient evidence to confirm the statement of Jack

${ }^{143}$ T. Pramanik, S. Pramanik, R. Chanda, "Postage stamps as a health promotion tool in the Nepalese Community", Eastern Mediterranean Health Journal, 200; 6: 723-733.

144 Jean-Jacque Lefrère, Bruno Daric, "Transfusion, blood donation and postage stamps: a worldwide review", Transfusion, 2010; 50: 1838-1848.

${ }^{145}$ Sarper Gursu, Timur Yildrim, Vedat Sahin, Emine Koc, “Art in science: orthopaedics through philatelic material”, Clinical Orthopaedics and Related Research, 2013; 471: 3755-3759.

Turkan Gursu, Alpher Eraslan, “Obstetrics and Gynecology on postage stamps: a philatelic study", Authorea 2020: DOI: 10.2254/ au.158379544.

Marc Shampo, Edward C. Rosenow, "A history of tuberculosis on stamps: Chest: 2009; 136: 578-582.

${ }^{146}$ Harlan J. Strauss, Politics, psychology and the postage stamp, State College, Pennsylvania: American Philatelic Society, 1975: 162, 178-179. Ibid 16-17.

${ }^{147}$ Frank Nuessel, "Postage stamps: a pedagogical tool in the second language classroom”, Mosaic: A Journal for Teachers, 1996; 3: 12-17. 
Child that "postage stamps have impact". ${ }^{148}$ There is now much evidence from research to support Child's statement. ${ }^{149}$

In light of the changing concepts of health promotion that have occurred over time, as discussed above, and the changing views expressed, the question then arises as to whether health promotion/ education stamps have also changed with time. While the stamps primary function is the pre-payment of postage, its secondary function, as a mass medium, can be to convey health information to the public or one of many other suitable topics. In light of the changing models of health promotion, the question then arises as to how postage stamp design has adopted or changed. It would appear that the earlier stamps, while raising topics on health matters, presented them as an historical or information matter rather than a true health educational entity as perceived currently. In other words, the traditional stamp promotes individuals with information to make better choices, while the newer varieties use their media facilities to alert the policymakers to the need for social change. The earlier health related stamps are characterised by those issued by Egypt and Brazil in the 1950s and early 1960s, such as those on the topic of schistosomiasis (Bilharziasis). Their design is dominated by a celebration of the pioneer doctors, such as Bilharz and da Silva, but do indicate the causative organism of this infectious disease (see figure 62). Similar stamps, of the same period, from India, Switzerland and Brazil (leprosy), and Monaco (multiple sclerosis) are included in the same figure to further illustrate these features.

\footnotetext{
148 Jack Child, Miniature messages, Durham: Duke University Press, 2008: 1.

149 Dennis Altman, Paper ambassadors: the politics of stamps, North Ryde, Australia: Angus and Robertson, 1991. Frank Nuessal, "Territorial and boundary disputes depicted on postage stamps", Studies in Latin American Popular Culture, 1992; 11: 123-141.

Bill McAllister, “House resolution would condemn Mexico's Memin stamp as racist", Linn's Stamp Monthly, 2005. August: 35.
} 

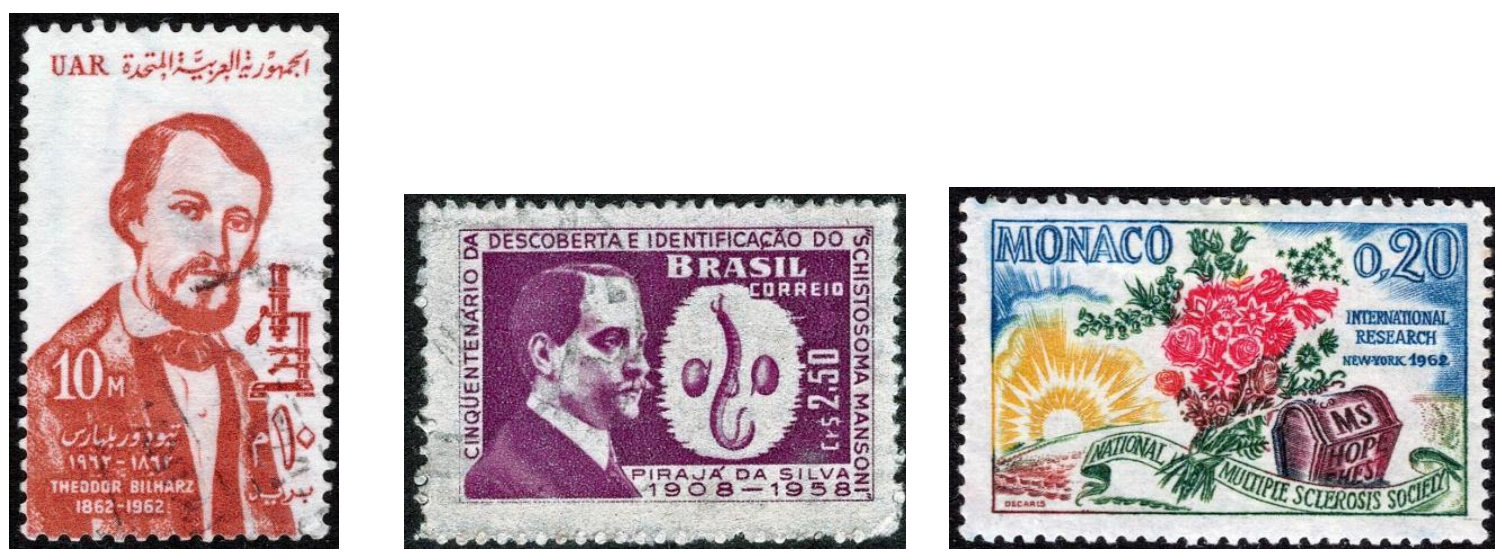

Figure 62. Bilharzia infection, Egypt (UAR), 1962, Brazil, 1958, Multiple sclerosis, Monaco, 1962.
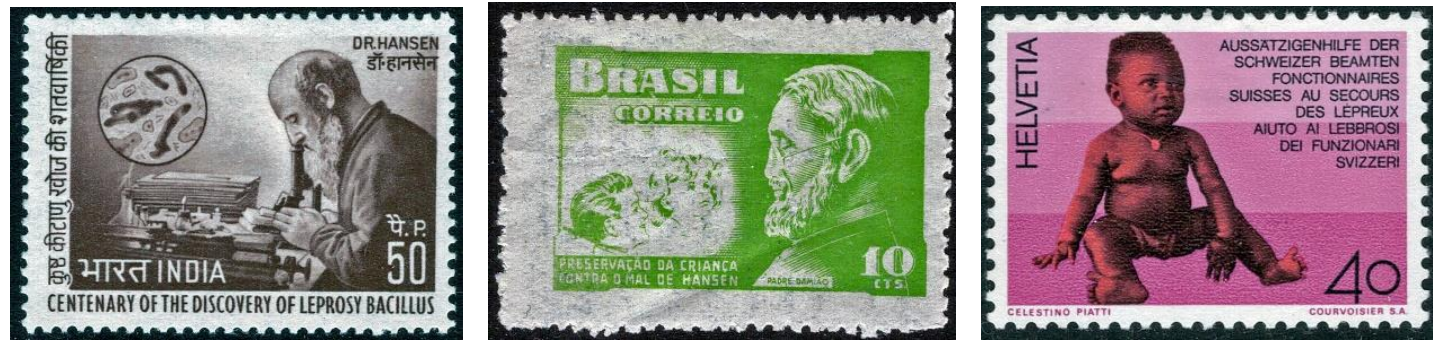

Figure 63. Leprosy, India, 1973, Brazil, 1958, Switzerland, 1976.

By the late 1970 s to the 1980 s relative stamps became more educational in content and early health promotion is becoming evident. Figure 67 indicates examples related to eye health drawn from a variety of countries. The stamps now show the anatomy of the eye and warn about the dangers of river blindness (onchocerciasis) and educate the people as to the surgery available in the form of corneal transplants. The stamp illustrated from Swaziland, in particular, draws attention to the association of malnutrition and eye disease (see figure $67)$. 

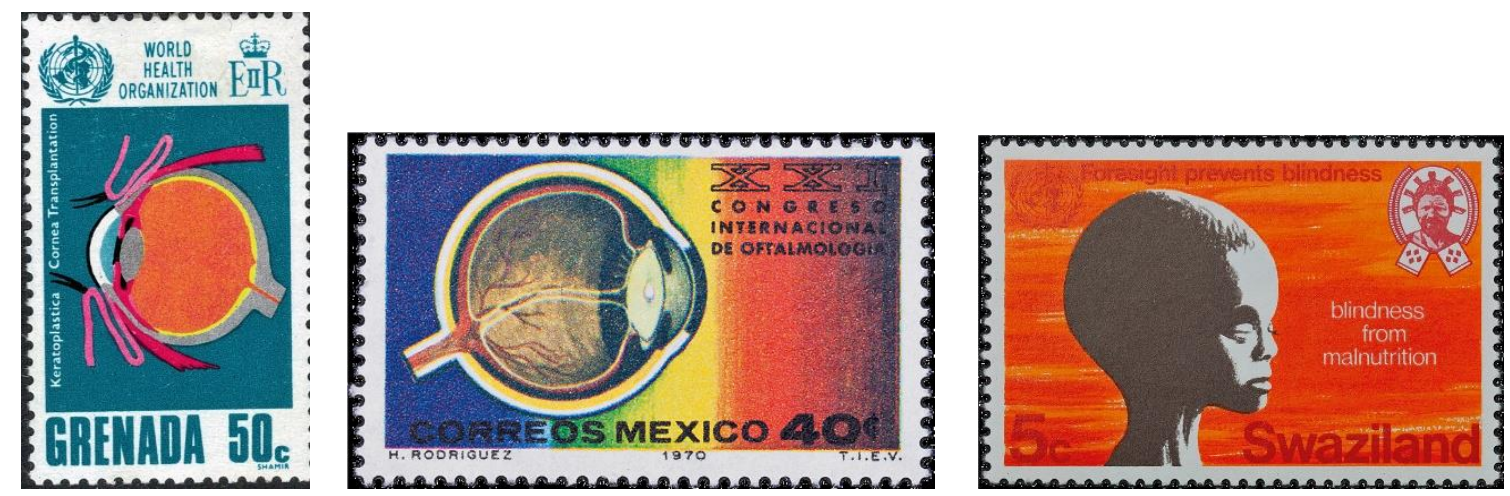

Figure 64. Corneal transplantation, Grenada, 1968, eye anatomy, Mexico, 1970, blindness and malnutrition, Swaziland, 1976.
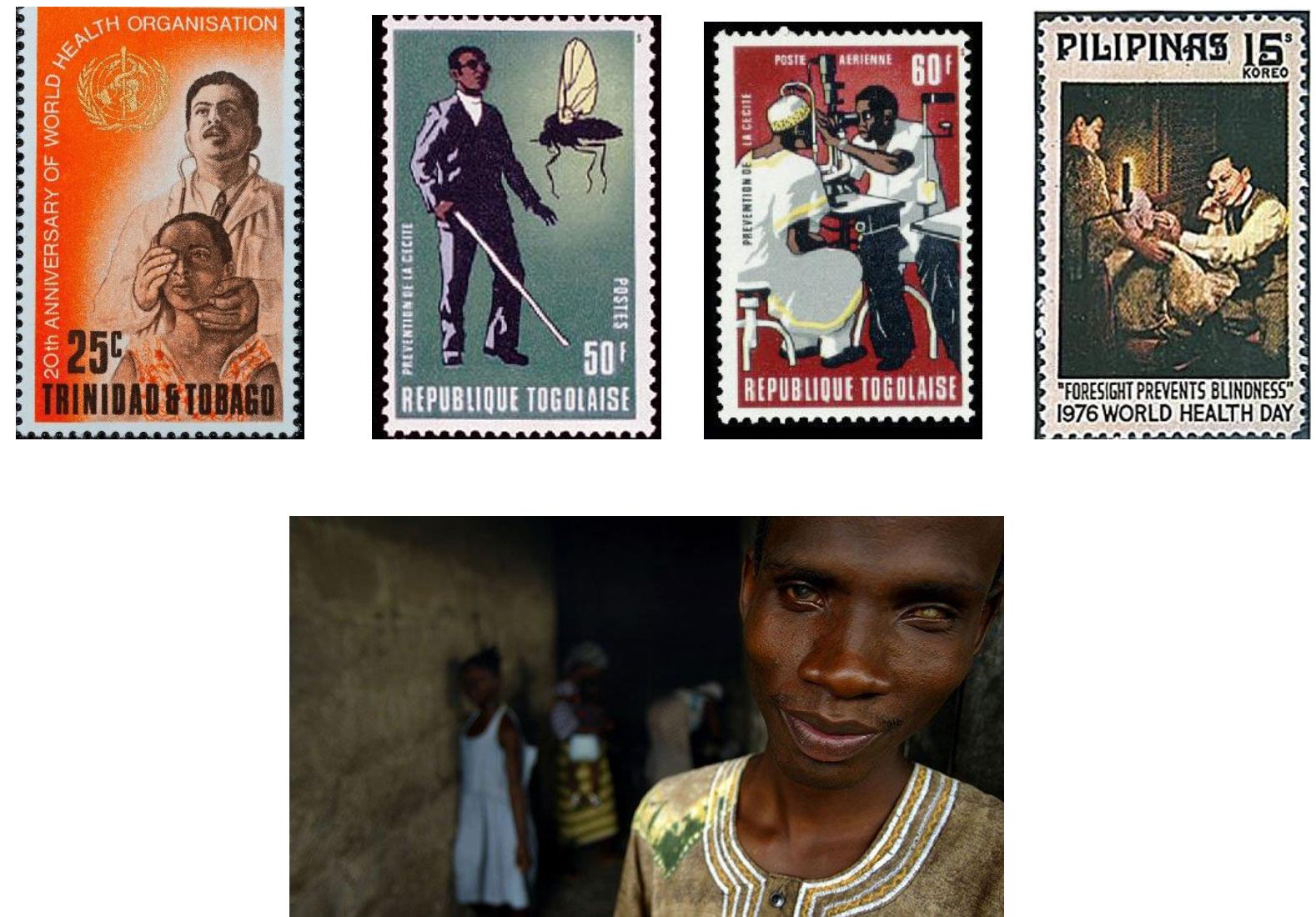

Figure 65. Eye examination, Trinidad and Tobago, 1968, river blindness and eye examination, Togo, 1976, "Foresight prevents blindness", Philippines, 1976, photograph of woman with river blindness. ${ }^{150}$

Between the 1980s and the end of the century, more stamps were produced of a health educational type, often with a text message and an improved technical presentation. Figure

\footnotetext{
150 River blindness: accessed 15/9/2020

https://www.everydayhealth.com/infectious-diseases/onchoceriasis-river-blindness/
} 
68 shows a first day cover of 1995, showng radiological equipment and the advances in radiology over the previous hundred years. Further explanatory information about the newer diagnostic tests in medicine is provided in British stamps and these are also shown in this figure. Finally, the introduction of a cartoon-like stamp conveying a health message is demonstrated in an Australian stamp of the period. This typified the move to non-traditional forms of stamp format and these were often used to make more of an imp.
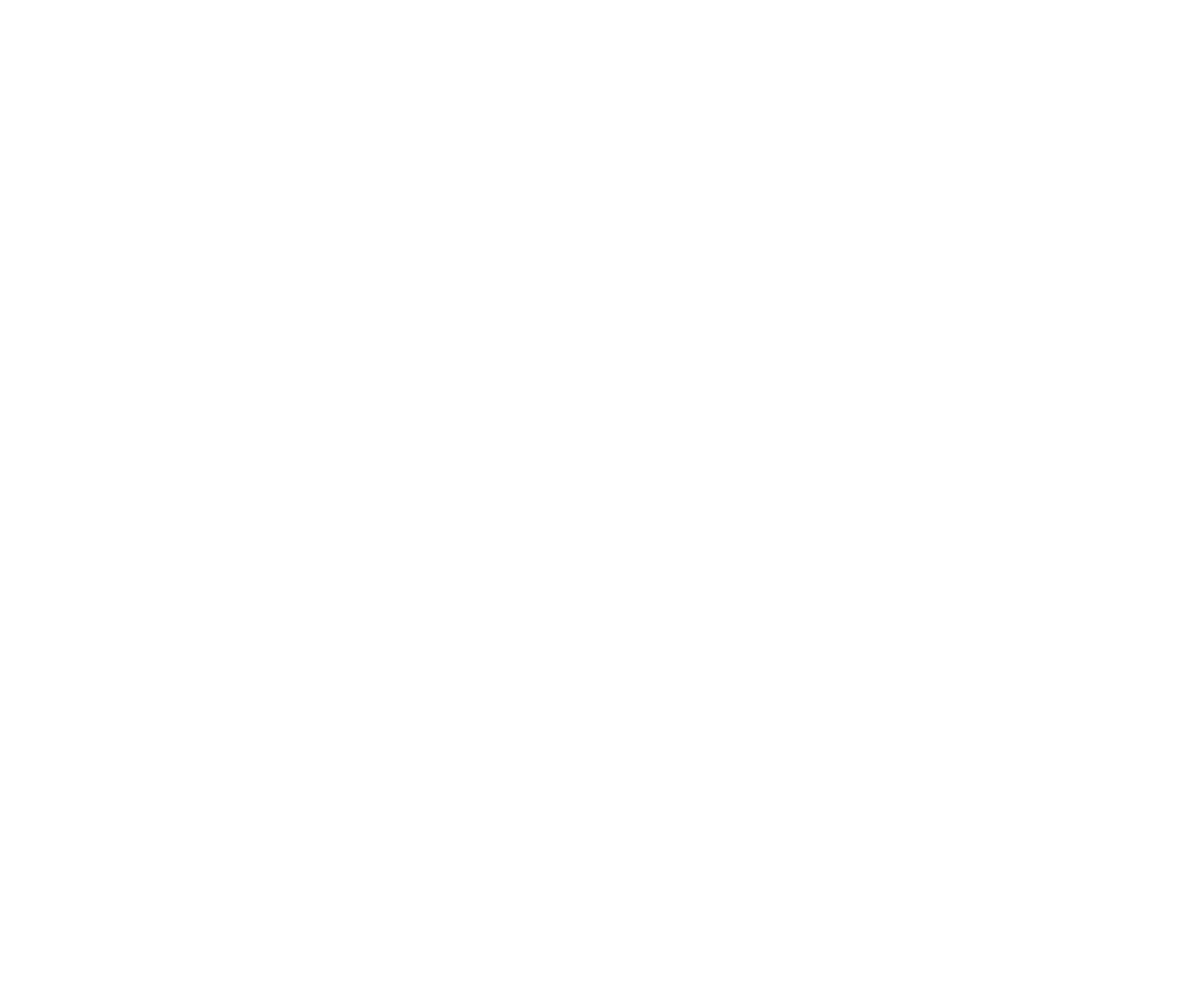

Figure 66. First day cover on radiological advances, Malaysia, 1995, Magnetic resonance imaging (MRI), United Kingdom, 1994, Ultrasound imaging, United Kingdom, 1994, Community health, Australia, 1990.

In the first two decades of the $21^{\text {st }}$ century, the boldness of design and the use of bright colours has created striking images for health promotion and education stamps. Figures 15 and 26 are examples of these newer stamps. Figure 67 shows further examples of recent 
stamps from this present era. Indeed, stamps continue to keep up to date on health matters with the current major pandemic of covid 19 viral infection being the subject of recent issues.
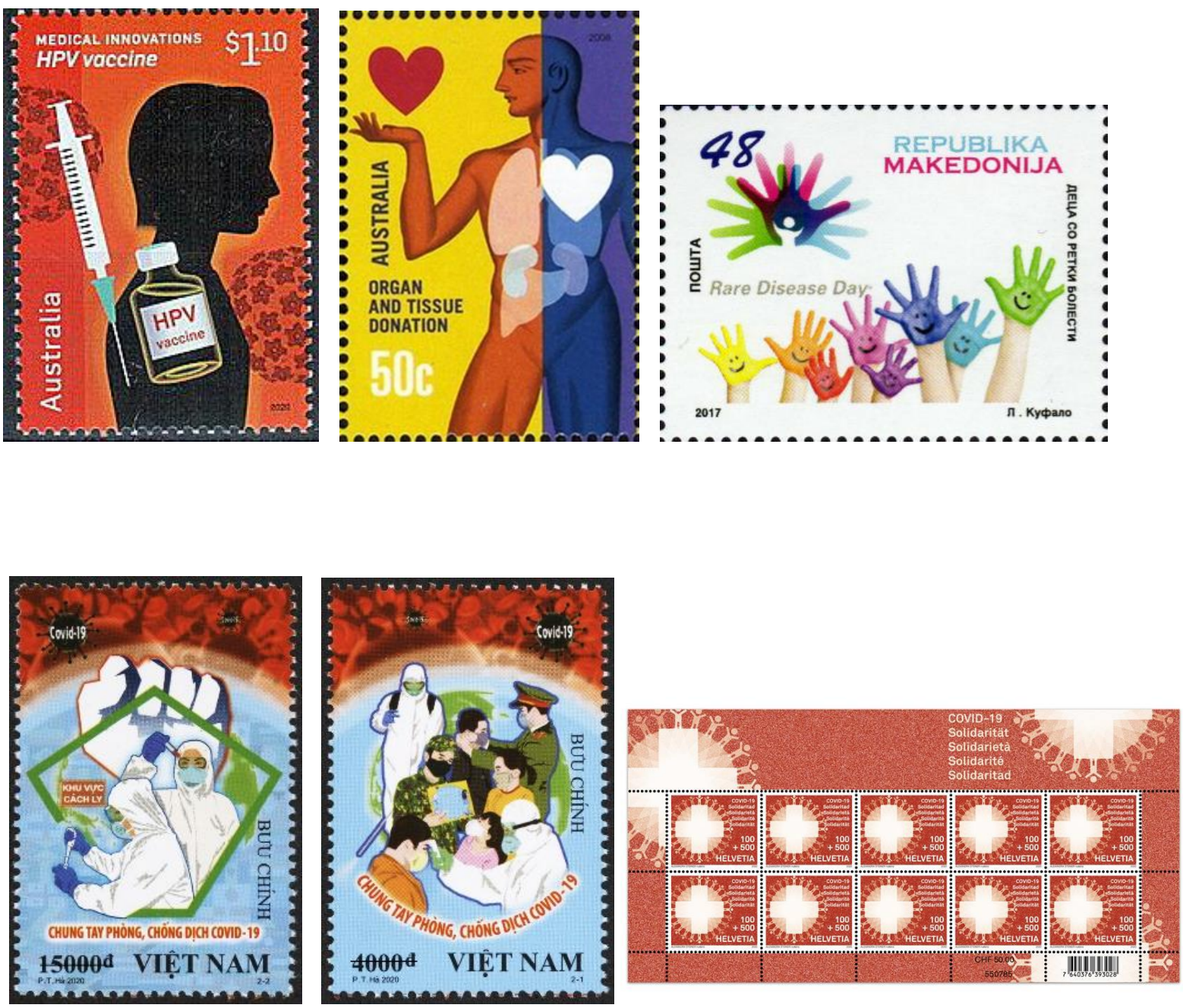

Figure 67. Health topics from Australia, Herpes virus immunisation and organ transplantation, 2020, 2008, rare disease awareness, Macedonia, 2017, Covid 19 protection and masking, Vietnam, 2020, solidarity against covid 19 virus, Switzerland, 2020.

In this chapter a brief outline of the definition of health promotion has been presented. Under the umbrella title of health promotion are included terms such as 'health education', 'health advocacy' and 'disease prevention'. Several models of health promotion exist, and it is argued here that the model referred to as 'media advocacy' appears to have the best fit for the position of the postage stamp in its role as a mass medium. Postage stamps are targeted at a specific local population as well as benefitting, at times, wider audiences. Their 
success stems from the fact that they are, in general, directed at specific communities with specific health needs and problems. The use of the postage stamp as a medium, avoids the criticism that only advantaged people receive the message, as postage stamps are ubiquitous in nature. The postage stamp has changed in format over time due to improvements in its technical production, but it does also appear that the way the health messages are presented over time has also changed. There is less concentration on important health individuals and more on the health advances available in term of diagnosis and treatment. It has been shown in earlier chapters that with individual diseases, such as malaria, there is a direct educational message given regarding management and prevention of these diseases. In addition, great support for the health benefits of breast feeding and its normality are presented in stamps.

An important paper by Hanlon et al., is relevant to the argument that health-orientated stamps have adapted to meet the changing needs and attitudes to health education and promotion over time. ${ }^{151}$ In their paper the authors have proposed that there have been several phases of public health improvement since the Industrial Revolution. This is as a result of major rethinking on the nature of health and about society itself. These changing views have been reflected in the subject material of health-orientated stamps and have reflected these 'waves' of change over time. The earliest phase, up to 1900 , contains the classical public health improvements such as in water purity and sanitation. The stamp of India shown figure 68 is typical of these issues. The period to 1950 forms the second wave and represents the scientific advances in health and examples of these can be found in figure 63. The described third wave lasted until 1980 and reflected the emergence of the welfare state, national health systems and the introduction of universal education. Here again, postage stamps have featured these changes and examples from New Zealand and the United Kingdom are shown in figure 68. The fourth wave from 1960 until 2000, features health care intervention to prolong life, yet at this time there is developing evidence of the emergence of social inequalities in health. Figure 66 indicates examples of the first aspect. Hanlon et al., now propose a fifth wave, in which subjects such as obesity, social inequalities in health and emotional and mental health reflect the overwhelming of society. Since the publication of their paper, the covid19 pandemic has struck the world associated with great

${ }^{151}$ P. Hanlon, S. Carlisle, M. Hannah, D. Reilly, A. Lyon. "Making the case for a 'fifth wave' in public health", Public Health, 2010; 125: 30-36. 
morbidity and mortality. Health related stamps are already associated with these changes. Figure 66 gives an example of an Australian stamp promoting a dietary warning in the prevention of obesity. Figure 68 includes example of mental health stamps intended to reduce the stigma still associated with these conditions.

Health is now regarded as a diverse and complex topic determined by political, social, and environmental factors. ${ }^{152}$ The United Nations has recognised this complexity by establishing seventeen sustainable developmental goals (SGD). ${ }^{153}$ While goal three specifically relates to health, it is clear that all seventeen goals impinge upon health status in its widest sense. The relevance of this fact, to health education and promotion through postage stamps, suggests that any promotion of the SGDs by postage stamps is valuable also in the promotion of health and wellbeing. It is proposed here, that while the concepts of what constitutes health have changed, it follows that what, in future, constitutes a 'health stamp' would also change and that any stamp that promotes one or more of the SGDs could now be regarded as a 'health stamp' in its new and widest sense.

The ever-broadening definition of health permits a wide range of new approaches to health education and promotion. Devon Greyson et al. warn that ethical problems may be associated with health intervention using new technologies. ${ }^{154}$ Postage stamps are free of these problems but are clearly less effective in achieving the goals of health promotion. ${ }^{155}$ Health education via stamps requires to adjust to these changes, hence formats must change to meet these needs. However, many have doubted that postage stamps in their present form of an adhesive label have a future at all. As discussed above, their topics and delivery methods, to date, have changed. The future primary function of the stamp is specifically in doubt, hence there would be a loss of their secondary role also. Previously when a health education stamp or other stamps were issued, many extra sheets, in excess of postal needs, were printed. These stamps were intended for the philatelic market in mind as a source of revenue for the postal systems. Within the last twenty years there has been a

\footnotetext{
152 Ilona Kickbush, "Addressing the interface of the political and commercial determinants of health", Health Promotion International, 2012; 27: 427-428.

J. Hope Corbin, "Health promotion research in the United Nations post 2015 agenda", Health Promotion International, 2015; 30: 1-4.

153 Development goals: https://sdgs.un.org/goals accessed 6/2/2021.

${ }^{154}$ Reference to information technologies, computer science theories and methods, surveillance programs and digital health promotion.

155 Devon Greyson, Rod Knight, Jean A. Shoveller, “Ethics, effectiveness and population health information interventions: a Canadian analysis", Health Promotion International, 2019; 34: 501-509.
} 
decline in philately as a hobby and hence a decline in extra revenue. Currently, to compensate for this fall in revenue, many stamp issues are being produced with secondary subjects aimed specifically at other special interest groups, rather than at the philatelic market. ${ }^{156}$ Stamps depicting Star Wars topics are aimed at the aficionados of the Star Wars films and others to Dr. Who enthusiasts. This, of course, bodes badly for any future stamps with a political, social or health topic. All is not lost however, as of late there are reports of an emerging of renewed interest in postage stamps as a hobby by Millennials and this is probably related to increasing affluence in this group. ${ }^{157}$ Their interest in the topics of stamps may be directed at social topics rather than pop stars or footballers. The Wall Street Journal has also reported that stamp collecting is also back in vogue, while Forbes Magazine advocates for stamps as a good financial investment at the current time. ${ }^{158}$ These facts have to be balanced against the warnings of Roland Burke, that stamps of a social and health nature must have a continuing active and efficient role in promotion. They must not be produced to serve as memorials to prior events, as he believes that the stamps from the United Nations for the Human Rights programme have become. ${ }^{159}$ It is concluded from this chapter, that as the academic concepts of what constitutes health promotion have changed, and the concepts of what constitutes public health have also altered, so have the formats and presentations of health-orientated stamps changed. However, their future as agents of health education is still in doubt.

\footnotetext{
156 Personal communication with the manager of postage stamps, New Zealand Post.

157 Nicole Mowbray, "Post Modern: why millennials have fallen in love with stamp collecting", The Observer, April 2020.

158 Richard Lehmann, "The future of philately", Forbes Magazine, 28 August 2016. Andrew Nelson, "Why stamp collecting is suddenly back in vogue", The Wall Street Journal, 5 June 2020.

159 Roland Burke, "Premature memorials to the United Nations Human Rights programme." History and Memory, 2016; 28: 152-181.
} 

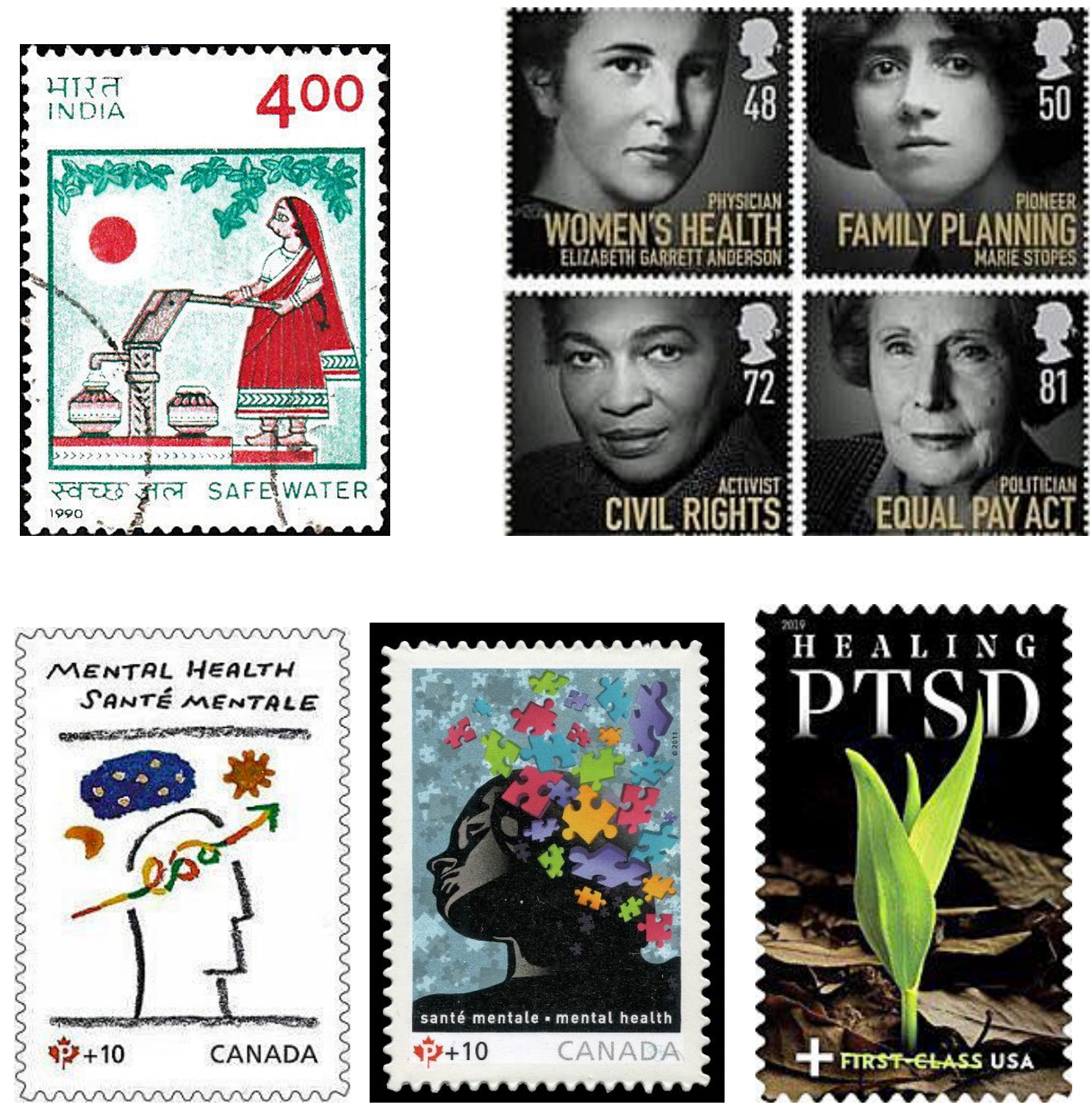

Figure 68. India (1990), safe water, United Kingdom (2008), aspects of health and social inequality, Canada (2009), mental health awareness, United States (2019), post traumatic stress syndrome. 


\section{Discussion}

Stamps which are the basis for the circulation of correspondence, facilitate communication while simultaneously expressing certain ideas and emotions through their own imagery. ${ }^{160}$

An important theme running through this thesis has been the demonstration that the study of postage stamps has relevance and forms a worthy topic for academic research. It has been shown here that many postage stamps have links and associations with the delivery of health promotion material, although, at best, this can only have minor standing in the overall delivery of health promotion and in achieving health equity, but this in itself does not exclude the determination of what their role and contribution has been.

Some of the first observations regarding the role of postage stamps in health promotion and education came from the papers of Davies and that of Marsden, in the 1980s. They both saw great potential in the use of the postal services, and the mail specifically, as a means of delivering health information. ${ }^{51}$ Subsequently, there have been many papers that have discussed the relevance of postage stamps in illustrating the work, history and scope of the many specialties involved in health care. ${ }^{161} \mathrm{~A}$ specific paper, referred to earlier, is that of Pramanik et al., who reported the use of postage stamps designed for health promotion in Nepal. They concluded that postage stamps, with pictures and slogans, to promote different aspects of health, were a useful method to inform the general public about health problems and disease prevention. They made reference to the high incidence of cataract causing blindness in Nepal and illustrated this with a Nepalese stamp showing an eye with a cataract (see figure 70). ${ }^{162}$ Further, Utpal Sanyal outlined the importance of 'information' in fighting AIDS (Acquired Immuno-Deficiency Syndrome) and described the role that postage stamps can play in the information campaign to create awareness of the disease and it sequelae. Sanyal illustrated his paper with images of stamps from thirty-seven countries, a relevant example from Lesotho is shown in Figure 69 that carries an important message.

${ }^{160}$ K. Evans, "The argument of images: historical representation in Solidarity underground postage (1981-87)", American Ethnologist, 1992; 19: 749-767.

161 James M. Dunlop, “Medical Nobels - stamps of genius”, British Medical Journal, 1988; 297: 1674. L. F. Hass, "Paul Ehrlich (1854-1915) and Emil Adolf von Behring (1854-1917)", Journal of Neurology and Neurosurgery, 2001; 70: 678.

Albert Chudley, "Genetic landmarks through philately - hereditary breast cancer', Clinical Genetics, 1999; 55: 416-418.

162 T. Pramanik, S. Pramanik, R. Chanda, "Postage stamps as a health promotion tool in the Nepalese community", Eastern Mediterranean Health Journal, 2004; 10: 442-444. 
Later publications began to examine the role that stamps may have in changing peoples' views and attitudes to health problems. Swan et al. examined the role of postage stamps
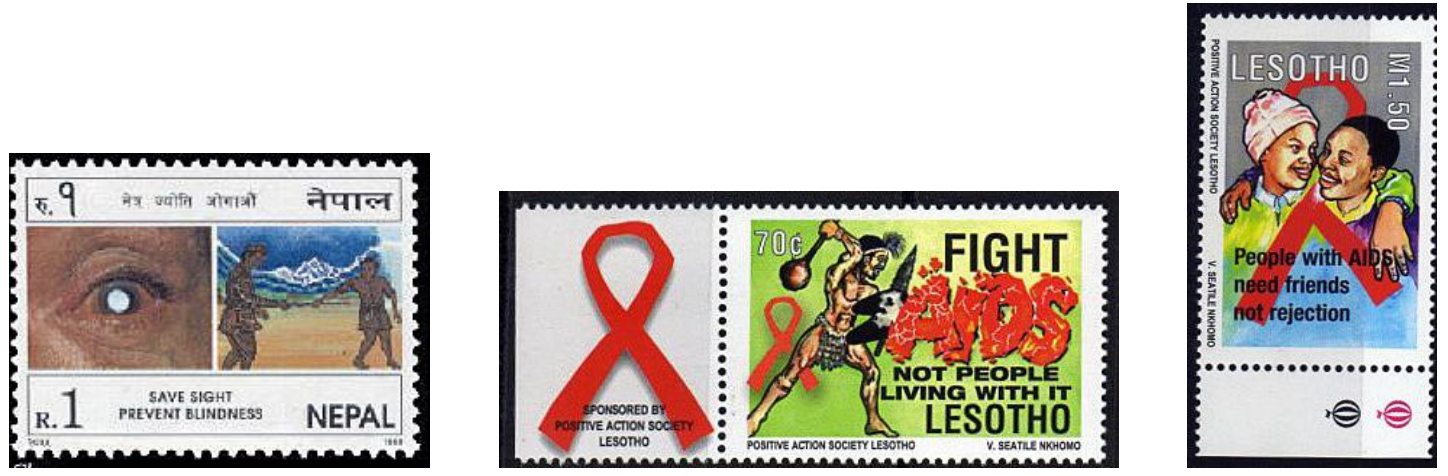

Figure 69. "Save sight prevent blindness", Nepal, 1998, "Fight AIDS, not people with AIDS" and "People with AIDS need friends not rejection", Lesotho, 2001.

in the field of disability and concluded "postage stamps contribute to the visual culture of disability and national and international discussions on disability" and that they received "public attention and changed public consciousness". ${ }^{163}$ Evidence was now accumulating as to the potential value of stamp use in health promotion.

A publication which attempted to review the ideology behind the role of postage stamps and health-promoting messages is that of Robin Kearns et al. ${ }^{81}$ They determined that postage stamps possess "agency to deliver and direct diverse public health messages". They further added that stamps have adaptiveness and their ability to support changing views and concepts of what constitutes health and what constitutes health promotion. This is certainly in keeping with the arguments put forward in Chapter 8.

However, the main thrust of this paper is directed at the role of New Zealand children's health stamps from 1929-2016. As previously discussed, these stamps are not health promotion stamps but merely semi-postal charity stamps raising funds for camps for children with a wide range of social and health problems. The stamp illustrations presented in their paper, such as pied stilts, butterflies and fish are not images regarded, in this thesis, as being health promoting representations and carry no texts other than 'health'. There are two minor and one major exception to this statement in the stamps of 1929, 1932 and 2015, and these were discussed in Chapter 2. The 2015 stamp can be regarded as a true health

${ }^{163}$ Geoffrey Swan, Teresa Meade, J. Douglass Klein, David Serin, "Licking disability: reflections on the politics of postage stamps", Radical History Review, 2006; 6: 228-232. 
stamps as defined in Chapter 3. Kearns et al. also include stamps issued to celebrate the Red Cross and the World Health Organisation to support their argument, again by the definition developed here, these are all irrelevant as health promotion stamps and simply praise the work of these organisations. The authors claim that the changes of image focus from health topics in New Zealand health stamps is "an eco-political orientation of health promotion which emphasises the natural world".

Finally, while Kearns et al. argue that letter postage is in decline and hence stamps, the reality is that postage stamp numbers have multiplied exponentially over recent years as have the number of issuing agencies and this pattern is likely to continue in this way. This view is supported by recent work by Christopher Yardley who has shown that the actual sales of stamps in the United Kingdom and Australia have increased despite the fall in letter postage. ${ }^{164}$ Other outlets for the sale of stamps have been shown to be unrelated to their postal function. ${ }^{73}$

The first action in addressing the research question was to define what actually constitutes a postage stamp from a postal label, such as a Christmas tuberculosis label. ${ }^{165}$ Having done this, it was necessary to distinguish further the term 'health stamp', from one classified as a 'true health promotion stamp' for the purposes of this research project. On examination of the available stamp designs, it was recognised that there was a vast number of postage stamps issued whose secondary function had been related to the large range of health topics that exist. The key action was to determine which of these issues could be classified as true health promotional and /or educational issues. The early chapters then address these issues and clarify what was actually being defined and measured.

In view of the large numbers of true health stamps, so determined, it was necessary to select a sample of these health topics to help answer the research question. The topics selected for study, however, do represent a wide spectrum of health, social and welfare issues viz. physiological state/ health benefit (breast feeding), infectious disease (malaria), social and behavioural issue (smoking), preventative disease (immunisation), as well as references to stamps relating to cancer detection.

\footnotetext{
${ }^{164}$ Christopher B. Yardley, The representation of science and scientists on postage stamps, PhD Thesis, Australian National University, 2013.

${ }^{165}$ E. Sanitize, R. Shengelia, "First partnerships to fight tuberculosis", Clinical Research and Bioethics, 2015; 6: 5- 10. James MacKay, Philatelic terms illustrated, London: Stanley Gibbons Ltd., 2003: 25.
} 
The next task was to relate the stamps of the selected topics to the concept of health promotion. What constitutes health promotion seemed at first a simple question but is, in reality, a much more complex issue. The guiding definition used has been that of the Ottawa Conference and Charter on Health Promotion. ${ }^{2}$ The term 'health education' has been used along with health promotion to cover the earlier postage stamp illustrations, while fully accepting that education lies within the spectrum of health promotion as now defined. It has been argued in Chapter 8 that the strategy of health promotion called 'media advocacy' most closely fits the position of the postage stamp in its secondary role as a mass medium. This model seems to fit well for stamps directed at discouraging tobacco use and those promoting breast feeding. Where this model is less satisfactory is in situations in which educational information is being presented visually or in text in the areas of infectious disease or immunisation. While the receivers of the information may find it useful, the more vulnerable people such as those in the lower socio-economic group or those with low health literacy may be less likely to act on the information. ${ }^{166}$ In this situation, 'nudge' theory may be more relevant or the community empowered model, as described by Mittelmark et al., may be more appropriate, which itself may employ media advocacy. ${ }^{3}$

When interpreting the visual data that formed the basis of the evidence presented to answer the research questions the form of analysis required is, clearly, vital as the data is of a qualitative nature. To place the stamps in context it was also necessary to provide information as to the events and background that prompted their production. This led to the need for a considerable amount of descriptive material to be included in each chapter. It is for this reason a case study format was used. The content analysis used was based upon the method successfully devised by Raento and Brunn and used by them in several peer reviewed publications on aspects of research which included the visual appraisal of postage stamps. ${ }^{167}$ Others such as Kearns et al. have followed their method of content analysis when researching aspects of postage stamp use. ${ }^{168}$

\footnotetext{
166 Pers-Anders Tengland, "Behavior change or empowerment: on the ethics of health-promotion", Public Health Ethics, 2012; 5: 140-153.

167 Pauliina Raento, "Communicating geopolitics through postage stamps: the case of Finland", Geopolitics, 2006; 11: 601-629.

Pauliina Raento, Stanley D. Brunn, “Picturing a nation: Finland and postage stamps, 1917-2000”, National Identities; 10: 49-75.

K. Covington, Stanley Brunn, “Celebrating a nation's heritage on music stamps: constructing an international community", GeoJournal 2006; 65: 125-135.

168 Kearns et al., 2019: 41.
} 
The analysis of the data used here was in keeping with the principles and questions laid out by Raento and Brunn and sought to identify the dominant theme of each stamp issue. Good examples of each of the analytical questions were found within the database. Problems related to child nutrition are given meaning by the display of weight and height charts and images of children failing to thrive. Cluster images and their meaningful association within images are found in malaria related stamps that show the source, prevention, outcome and treatment of malaria. Associations between clusters were found in those issues such as smoking cessation and infant feeding were advocacy was a common theme linking the two clusters. Finally, an excellent example of "invisibility" was found in the stamp format of the American issues for prominent people. The cigarette that was being smoked by the individuals in the original photographs, that formed the basis of the stamp, had been removed in the final stamp format. The additional questions posed in the methodology, but not part of the original design of Raento and Brunn, were all addressed in the text in the process of answering the research questions. Despite the above, it has to be recognised that there is limitation to this type of analysis in that despite every attempt to be fully trustworthy, there is an inherent subjectivity in the classification system. ${ }^{169}$ The role of the postage stamp comes into its full potential when it supports major health campaigns such as that described for the World Health Organisation malarial campaign, as is reported in Chapter 4. The health educational potential and the approach taken by many subscribing countries is described in some detail in this chapter. The fact that 140 countries participated in the production of specific anti-malarial stamps for the event, with many educational orientated issues, suggests the importance given by these authorities to the role that stamps can play. While the actual health campaign did not reach the level of success hoped for, this fact does not detract from the importance of the stamps' educational contribution. Many countries have continued to use stamps, subsequent to the end of the campaign, to inform their people about malaria, using a wide range of designs which are usually health promoting in content (figure 39).

In Chapter 5, the impact factor of the postage stamp is fully revealed. Many of the examples chosen have come from anti-tobacco campaigns, but they are equally as effective in antialcohol and other drug strategies, where the shock technique is also used (see figure 25).

169 Raento and Brunn,2005: 148. 
There is support in the research literature that the shock approach is effective in achieving its goal. However, sometimes subtle humour can be effective as shown in stamp from the Czech Republic and Denmark (figure 70). Overall, some 60 nations have produced over 100 anti-smoking stamps, so like anti-malarial stamps, they have been used widely and have established a clear role in the campaign against tobacco use. ${ }^{170}$
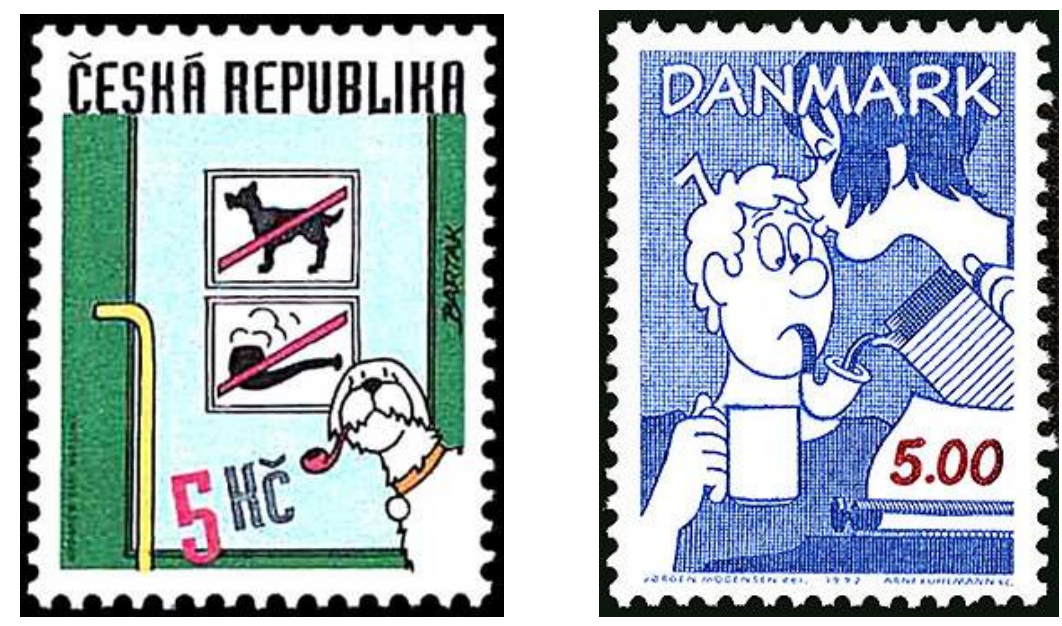

Figure 70. Humorous stamps against smoking, Czech Republic, 1999, cartoon by Miroslav Bartak, and Denmark, 1992.

The large number of stamps issued on the subject of immunisation against human disease has again required the need for some selection in what is to be presented. While several issues simply encourage immunisation against a number of common conditions, others are more disease specific (figure 48). Poliomyelitis was chosen as the subject here for further assessment because it is a good example of the success that can be achieved by immunisation in attempting to eliminate a disease world-wide. It is clear that stamps about poliomyelitis immunisation have been widely used and they have combined some historical information along with facts about the simplicity and methods of delivery of the vaccine. Earlier stamps have warned of the consequences of the disease if immunisation is neglected.

What impact do these stamps have in improving community levels of immunisation? Immunisation decisions are influenced by a host of factors and among them, it has been shown, that trust and public support for immunisation is important. Stamps are official

${ }^{170}$ Stamp use: https://csts.ua.edu/stamps/ accessed 11/9/2020. 
documents and may help instil trust. ${ }^{171}$ Additionally, information does influence the individuals decision to immunise their children, and this information must be by consensus and messages given in early pregnancy have been shown to be particularly effective. While the messages delivered by postage stamp are not going to be the key factor in immunisation decisions, they do provide some of the essential factors that have been shown to be influential in the decision to immunise or not. As discussed earlier, the postage stamp has been used to herald the success that vaccination has played in the elimination of smallpox and is now being used to introduce newer vaccines such as that used to prevent cervical cancer (figures 26,69 ).

The important subjects of childhood nutrition, growth and development have all been addressed in many stamp releases over many years. In particular, breast feeding has been well promoted in stamp designs, being presented generally as the normal and accepted method of feeding infants. However, direct promotion of breast feeding and the opposition to formula feeding has been featured in some issues (figure 57). It is observed in Chapter 7 , that there is a cultural difference in the way breast feeding is represented in stamp form, as there is resistance in some societies to the direct images of breast feeding. This observation may be important when encouraging women to breast feed when the society regards the images used to do this are culturally unacceptable.

The problem of malnutrition, in terms of low-calorie intake, is addressed by stamp issues from many low-income countries and in their rural populations. Postage stamps have been used frequently to stress the need for routine screening and assessment of infants and children. The regular weighing and growth measurement of infants has been a frequent theme in the stamps of many African countries. The development and growth of infants can be jeopardised by gastroenteritis and the introduction of oral rehydration therapy has been lifesaving and has revolutionised its management in resource poor countries. Health promoting stamps have been used to give information as to the use of these solutions and how they are reconstituted and encouragement to use them (figure 61). However, the

${ }^{171}$ Mary Nowlan, Esther Willing, Nikki Turner, "Immunisation and policies that affect immunisation coverage a summary review of literature", New Zealand Medical Journal, 2019; 132: 79-88. Yunmi Chung, Jay Schamel, Allison Fisher, Paula M. Frew, "Influences on immunization decision making among US parents of young children", Maternal Child Health Journal, 2017; 21: 2178-2187. 
making up of these solutions with safe water is another topic that has been the subject of stamp issues.

In terms of answering the research question of the part played by postage stamps in health promotion, it is clear that postage stamps have been used by almost all nations to convey health information and to encourage altered behaviours in relation to immunisation, infant feeding and smoking. Major health campaigns have been accompanied by special stamp issues. However, stamps are only one of the many ways that health information can be imparted, but it is the frequent exposure to the message, in everyday society, which may make it a unique method of imparting health information and a method of promoting health and wellbeing. The stamp issues may serve by mass media advocacy to bring pressure upon policy makers in such matters as immunisation and the tobacco industry, but yet have direct effect upon individuals in society to encourage the best feeding practices and infant care and create smoke-free environments. The fact that these stamps have impact is supported by empirical research and by their continued use over many decades. At best, they do play only a small part in the overall delivery of health promotion, but they have a unique way of reaching families and informing them. The data presented here, in the analysis of stamp issues, has also provided insight into health conditions that have been tackled, over time, and how they have been addressed. Further, this information can provide potential data for future research that could lead to analysis of public health priorities overall.

There now exists a paradox, in that stamp production, in terms of the number of releases, is increasing as is the number of stamps in each issue. At the same time the number of letters being posted is dramatically decreasing, due to email and other electronic communication. In countries like New Zealand and the United Kingdom, the commercialisation of the postal agencies has changed the motivation of these agencies to produce stamps that raise revenue in their own right. Such subjects as pop stars and other popular culture images now have preference to health topics. ${ }^{73}$ Stamps appealing to tourists as souvenirs of their visits not only help fund the post office, but the stamps are usually not even used for postal services. However, there are many countries that see value still in stamps and continue to 'stamp' out disease and social change by this method. 


\section{Conclusion}

Postage stamps have played a small but unique role in health promotion and education.

They have been used world-wide, using their secondary function, as a method of delivering health information and health advocacy directly to peoples' homes and workplaces to aid the population to make informed choices about their health care and welfare. The primary role of postage stamps has helped to fund this secondary role as miniature messangers of health information. 


\section{Bibliography}

\section{Primary Source Material}

Postage Stamps

Issues selected from 1897 to 2020 of health promotion and education and stamps used for illustration.

Afghanistan: 1961, 1962

Algeria: 1996

Andorra: 2006

Antigua and Barbuda: 1992

Argentina: 1956, 2001

Australia: 1967, 1990(2), 2008, 2020(4)

Austria: 2013

Bangladesh: 1988, 1996

Belgium: 1916(2), 1929, 1931, 1932, 1979, 1993, 2017

Bolivia: 1928, 2001

Bophuthatswana (South Africa): 1980, 1985

Bosnia: 2001, 2003

Botswana: 2011, 2013

Brazil: 1958, 1959

Brunei: 1968

Burkina Faso: 1986, 2010

Canada: 1951, 1962, 1971, 1991, 1998, 2005, 2009

Cape Verde Islands: 1990

Central African Republic: 2018, 2019

China: 1980

Columbia: 1962

Congo (Brazzaville): 1981

Costa Rica: 1981

Croatia: 1993

Cuba: 1957, 1962, 1973, 1984

Cyprus: 1963, 1995, 2013

Czechoslovakia: 1975, 1981

Czech Republic: 1999

Dahomey: 1942

Denmark: 1988, 1992

Djibouti: 1994

Dominican Republic: 1956, 1962

Dominica: 2000

Dubai: 1962

Egypt: 1962, 1976

El Salvador: 1990

Ethiopia: 1962, 1963

Equatorial Guinea: 1986 
France: 1962

Finland: 1947, 1955, 1967

Gabon: 1962, 1966

Gibraltar: 1968

Greece: 1966

Ghana: 1973(2), 2000

Grenada; 1968(2)

Guinea Bissau: 2013

Guyana: 1978

Honduras: 1943, 1960, 1998

Hungary: 1973

India: 1973(2), 1980, 1981, 1986, 1990, 2008

Indonesia: 1991

Iran: 1962, 1970, 2000

Israel: 1962

Italy: 1982

Jamaica: 1968

Japan: 1987

Jordan: 2001

Kenya: 2006

Kuwait: 1963

Lebanon: 2000

Lesotho: 2001

Luxembourg: 2003

Macedonia: 2017

Malawi: 2005

Maldives: 1980

Malaysia: 1975, 1995 (first day cover)

Mauritius: 1978, 1999

Mayotte: 2001

Mexico: 1939, 1962, 1970, 1995, 2005

Monaco: 1962, 1996

Morocco: 1962

Mozambique: 2013, 2015

Nepal: 1998

New South Wales: 1897

Netherlands: 1906

New Zealand: 1929, 1930, 1931, 1967, 2003, 2015

Nicaragua: 1937, 1973, 1987

Nigeria: 1962, 1984, 2004

Pakistan: 1982, 2009

Panama Canal Zone: 1962

Papua New Guinea: 1979, 1982

Paraguay: 1932

Peru: 1987

Philippines: 1959, 1962, 1972, 1976, 1978, 1980

Poland: 1948, 1971 
Portugal: 1980(2)

Portugal (Azores): 2003

Rwanda: 1970

San Marino: 2002

Saint Thomas and Prince Islands: 2009

Saint Vincent: 1987

Samoa: 1973

Saudi Arabia: 1997

Serbia; 2011

Sharjah: 1962

Sierra Leone: 2015

Solomon Islands: 1977, 2012

Somalia: 1962

South Korea: 1954, 1961, 1962, 2004

South Vietnam: 1962

Sri Lanka: 1982

Swaziland: 1976

Sweden: 1984

Switzerland: 2010

Tunisia: 1962

Syria: 1997, 1998

Taiwan: 1962

Tanzania: 1986, 1988, 2003, 2005

Thailand: 1977

Togo: 1914, 1962, 1976, 2000

Transkei (South Africa): 1979(2), 1991

Trinidad and Tobago: 1968

Turkey: 1961

Uganda: 1985(2)

United Kingdom: 1965, 1994(2), 2003, 2008, 2010, 2018

United Nations: 2010

United States of America: 1957, 1965, 1994, 1996(2), 1998, 1999(2), 2000, 2006(FDC), 2010, 2019

Upper Volta: 1970

Vanuatu: 1996

Vietnam: 2018, 2020(2)

Wallis and Futuna: 1981

Zambia: 1970, 1988

Zimbabwe: 1987(2)

Meghoot post cards, 2003, 2007 


\section{Secondary source material}

\section{Books}

Baum, F., The new public health, Oxford: Oxford University Press, 2016.

Billig, M., Banal nationalism, New York: Sage Publishing, 1995.

Child, J., Miniature messages, Durham: Duke University Press, 2008.

DiClemente, R., Raczynski, J., "The importance of health promotion and disease prevention", in Raczynski and DiClemente (eds) The Handbook of Health Promotion and Disease

Prevention, Boston: Springer, 1999: 3-9.

Gibbons, G., Stamps of the world, Ringwood, England: Stanley Gibbons Ltd., 2017.

Gibbons, S.G., Commonwealth and Empire stamps (1840-1970), Ringwood, England; Stanley

Gibbons Ltd., 2017 and United States of America, 2010.

Groepe, MA., Urbach, PDF., Hlongwana KW., et al., "Health promotion: from malaria control to elimination", South African Medical Journal, 2013; 103: 799-800.

Hutt, MSR., Burkitt, DP., The geography of non-infectious disease, Oxford: Oxford University Press, 1986.

Lutz, CA., Collins, JL., Reading National Geographic, Chicago: University of Chicago

Press,1993.

Mackay, J., The Guinness book of stamps, Middlesex: Guinness Publishing Ltd., 1988: 109-

110.

MacKay, J., Philatelic terms illustrated, London: Stanley Gibbons Ltd., 2003: 25.

McLintock, A.H. (ed.) "Mail services", An Encyclopaedia of New Zealand, Wellington:

Ministry of Culture and Heritage, 1966.

Ministry of Health, Health literacy review: a guide, Wellington; Ministry of Health, 2015.

Perry, C., Chhatralia, K., Damesick D., et al., Behavioural insights in health care, London: The Health Foundation, 2015.

Pierce, CS., Pierce on signs: writings on semiotic, ed. James Hooper, Chapel Hill: University of North Carolina press, 1991.

Rose, G., Visual methodologies: introduction to the interpretation of visual materials, London: Sage Publications, 2001.

Scott, D., European stamp design: a semiotic approach to designing messages, London:

Academy Editions, 1955.

Shafir, E., History and perspective of diabetes illustrated by postage stamps, London: Freund Publishing Ltd.,1999.

Stoetzer, C., Postage stamps as propaganda, Washington: Public Affairs Press, 1953: 1.

Strauss, H., Politics, psychology and the postage stamp, State College, Pennsylvania: Fortyfirst American Philatelic Congress Book, American Philatelic Society, 1975; 162: 178-179.

The Len Jury New Zealand Stamp Catalogue, New Plymouth: Philatelic Distributors Limited, 2019.

Thaler, RS., Cass, R., Nudge: improving decisions about health, wealth, and happiness, London: Penguin Books, 2008.

Wallack, L., Dorfman, L., Jernigan, D., Themba, M., Media advocacy and public health: power for prevention, Newbury Park, California: Sage, 1993.

Watson, J., The Stanley Gibbons book of stamps, Ringwood: Stanley Gibbons Publications Ltd., 1990: 74. 
Wolfe, R., It's in the post: the stories behind New Zealand stamps, Nelson: Craig Potton Publishing, 2010: 72-79.

World Health Organization, Global strategy for malaria, 2016-2030, Geneva: WHO Press: 2015.

Zeyen, T., The story of ophthalmology told by postage stamps, Clermont Ferrand: Laboratoires Thea, 2019.

\section{Journals}

Afshar, A., "A brief Iranian medical history through commemorative postage stamps", Archives of Iranian Medicine, 2010; 13: 161-165.

Altman, D., Paper ambassadors: the politics of stamps, North Ryde, Australia: Angus and Robertson, 1991.

Baskota, P., The New Indian Express, $8^{\text {th }}$ March 2018.

Belfield, CR., Kelly, IR., "The benefits of breast feeding across the early years of childhood", The Journal of Human Capital, 2012; 6: 251-277.

Bengtsson, M., "How to plan and perform a qualitative study using content analysis", NursingPlus Open, 2016; 2: 8-14.

Berry-Cabal, CS., "DDT and silent spring: fifty years after", Journal of Military and Veterans Health, 2011; 19: 19-24.

Bishon, M., "Record breaking stamp raises sticky questions", Cancerworld, 2006; NovemberDecember: 32-35.

Brown, A., "Personal experience in the malarial eradication campaign, 1955-1962", Journal of the Royal Society of Medicine, 2002; 95: 154-1962.

Brunn, SD., "Stamps as messengers of political transition", Geographical Review, 2011; 101 : 19-36.

Brunn, SD., "Stamps as iconography: celebrating the independence of new European and Central Asian states", GeoJournal, 2000; 52: 315-323.

Corbin, JH., "Health promotion research in the United Nations post 2015 agenda", Health Promotion International, 2015; 30: 1-4.

Brunn, SD., "A geopolitical and geovisualization challenge: increasing awareness of global environmental changes through postage stamps". Natural Resources. 2017; 8: 130-158.

Burke, R., "Premature memorials to the United Nations Human Rights programme." History and Memory, 2016; 28: 152-181.

Buturovic, Z., Ignjatovic, S., "Attitudes towards breastfeeding and breastfeeding practice: lack of support for breastfeeding in public as a factor in low breastfeeding rates", Journal of Applied Health Science,2017; 3: 137-143.

Child, J., "The politics and semiotics of the smallest icons of popular culture: Latin American postage stamps", Latin American Research Review, 2005; 40: 108-137.

Chudley, AE., Haworth, JC., "Genetic landmarks through philately - haemophilia", Clinical Genetics, 1999; 56: 279-281.

Chudley, A., "Genetic landmarks through philately - hereditary breast cancer', Clinical Genetics, 1999; 55: 416-418.

Chudley, AE., "Genetic landmarks through philately - a brief history of diabetes mellitus", Clinical Genetics, 1999; 55: 233.

Chung, Y., Schamel, J., Fisher, A., Frew, PM., "Influences on immunization decision making 
among US parents of young children", Maternal Child Health Journal, 2017; 21: 2178-2187. Davies, MK., "Health promotion with the help of the world's postal services", World Health Forum, 1989; 10: 69-180.

Davies, MK., "Health messages on postage stamps", World Health Forum, 1992; 13: 48-49. Davies, MK., Mayne, AJ., "Infant monitoring", Archives of Disease in Childhood, 2001; 84: 386.

Davis, MK., Mayne, AJ., "Stamps in paediatrics", Archives of Disease in Childhood, 2000; 82: 20.

Dawson, KP., "Paediatric philately: gastroenteritis and rehydration", Middle East Paediatrics, 2004; 9: 29.

Deans, P., Dobson, H., "Introduction: East Asian postage stamps as socio-political artefacts", East Asia, 2005; 22: 3-7.

Dunlop, JM., "Medical Nobels - stamps of genius", British Medical Journal, 1988; 297: 1674. Editorial, "What is health promotion?" Journal of Public Health Policy, 1986; 7: 147-51. Editorial, How a US postal stamp helped fund a pivotal study on breast cancer, Associated Press, June 4, 2018.

Editorial, "The double burden of malnutrition", The Lancet, 2019; 395: 5.

Ekker, C., "Stamps as a unique primary research materials", Topic Time, 1969; 20.5: 40-41. Evans, K., "The argument of images: historical representation in solidarity, underground postage 1981-87", American Ethnologist, 1992; 19: 749-767.

Fern, F., "Tokdo or Takeshema?" Stanford Journal of East Asian News, 2005;5: 78-83.

Fillinger U., Sombroek, H., Majambere, S., et al., "Identifying the most productive breeding sites for malaria mosquitoes in The Gambia", Malaria Journal, 2009; 8: 62.

Fong, G., Hammond, D., Hitchman, S., "The impact of pictures on the effectiveness of tobacco warnings". Bulletin of the World Health Organization, 2009; 87: 640-643. Friedman, T.R., "A framework for public health action; the health impact pyramid", American Journal of Public Health, 2010; 100: 590-595.

Gentleman, D., "The design and production of postage stamps", Journal of the Royal Society of Arts, 1974; 122: 431-449.

Gendall, P. Hoek, J. Gendall, K., "Evaluating the emotional impact of warning images on young adult smokers and susceptible non-smokers", Journal of Health Communication, 2018; 23: 291-298.

Greenwald, RA., "The postage stamp as a messenger", Tobacco Control, 1992; 1: 87-88.

Greyson, D., Knight, R., Shoveller, JA., "Ethics, effectiveness and population health information interventions: a Canadian analysis", Health Promotion International, 2019; 34:

501-509.

Groepe, MA., Urbach, PDF., KW Hlongwana KW., et al., "Health promotion: from malaria control to elimination", South African Medical Journal, 2013; 103: 799-800.

Gursu, S., Yildrim, T., Sahin, V., Koc, E., "Art in science: orthopaedics through philatelic material", Clinical Orthopaedics and Related Research, 2013; 471: 3755-3759.

Gursu, T., Eraslan, A., "Obstetrics and Gynecology on postage stamps: a philatelic study", Authorea, 2020: DOI: 10.2254/ au.158379544.

Hamza AM, El Rayah EL., "A Qualitative Evidence of the Breeding Sites of Anopheles arabiensis Patton (Diptera: Culicidae) in and Around Kassala Town, Eastern Sudan", International Journal of Insect Science, 2016; 8: 65-70.

Hanlon, P., Carlisle, S., Hannah, M., Reilly, D., Lyon, A., "Making the case for a 'fifth wave' in public health", Public Health, 2010; 125: 30-36. 
Hsieh, H-F., Sarah E. Shannon, SE., "Three approaches to qualitative content analysis", Quality Health Research, 2005; 15: 1277-88.

Hass, LF., "Paul Ehrlich (1854-1915) and Emil Adolf von Behring (1854-1917)", Journal of Neurology and Neurosurgery, 2001; 70: 678.

Howat, P., Cross, D., Maycock B., et al., "Towards a more unified definition of health promotion", Health Promotion Journal of Australia, 2003; 14: 82-84.

Isaacs, D., "Power of visual imagery", Journal of Paediatrics and Child Health, 2016; 52: 859860.

Jones, P., "Posting the future: British stamp design and the 'white heat' of a technological revolution", Journal of Design History, 2004; 17: 163-176.

Johnson, DR., "Malarial eradication and postage stamps", Mosquito News, 1961; 21: 79-83.

Kearns, RA., Coleman, T., Edmeades, J., “New Zealand children's health stamps: ideological artefacts linking health and place", Social Science and Medicine, 2019; 227: 38-46.

Kickbush, I., "Addressing the interface of the political and commercial determinants of health", Health Promotion International, 2012; 27: 427-428.

King, S., Pink Ribbons Inc.: breast cancer and the politics of philanthropy, Minneapolis: University of Minnesota Press 2008:61.

Kumar, S., Preetha, G.S., "Health promotion: an effective tool for global health", Indian Journal of Community Medicine, 2012; 37: 5-12.

Lee, P-W., Liu, C-T., Rampao, HS., do Rosario, VE., Shaio M-F., "Pre-elimination of malaria on the island of Príncipe", Malaria Journal, 2010; 9: 26.

Lefrère, J., Daric, D., "Transfusion, blood donation and postage stamps: a worldwide review", Transfusion, 2010; 50: 1838-1848.

Lehmann, R. "The future of philately", Forbes Magazine, 28 August 2016.

Limor, Y., Tamir, I., "The neglected medium: postage stamps as mass media",

Communication Theory; 2020: March; 1-15.

Lutschg, JH., "Anti-tobacco postage stamps", Tobacco Control, 1992; 1: 5-6.

Madsen, OA., "Health messages on everybody's mail", World Heath Forum, 1988; 9: 24-28.

Pan American Union, "The Americas through their postage stamps", Bulletin of the Pan

American Union, 1944; June: 356.

McAllister, W., "House resolution would condemn Mexico's Memin stamp as racist", Linn's Stamp Monthly, 2005. August: 35.

Ménard, J-F., "A 'Nudge' for public health ethics: libertarian paternalism as a framework for ethical analysis of public health interventions?" Public Health Ethics, 2010; 3: 229-238.

Mittelmark, D., Kickbusch, I., Rootman, I., Scriven, A., Tones, K., Health promotion, Elsevier Inc. International Encyclopedia of Public Health, volume 3, 2017.

Morris, D.M., Jenkins, G.R., 2018. "Preparing physical and occupational therapists to be health promotion practitioners: a call for action". International journal of Environmental Research and Public Health, 2018; 15: 392.

Najara, J., Gonzalez-Silva, M., Alonso, M., "Some lessons for the future from the Global Malarial Eradication Programme (1955-1969)", PLOS Medicine, 2011; 8: e1000412.

Najara, J. "Malaria and the work of the WHO", Bulletin of the World Health Organisation, 1989; 67: 229-243.

Nowlan, M., Willing, E., Turner, N., "Immunisation and policies that affect immunisation coverage - a summary review of literature", New Zealand Medical Journal, 2019; 132: 7988. 
Nuessel, F., "Postage stamps: a pedagogical tool in the second language classroom", Mosaic: A Journal for Teachers, 1996; 3: 12-17.

Nuessal, F., "Territorial and boundary disputes depicted on postage stamps", Studies in Latin American Popular Culture, 1992; 11: 123-141.

Nutbeam, D., "Evaluating health promotion - progress, problems and solutions", Health Promotion International, 1998; 13: 27-44.

O'Donnell, M., "Definition of health promotion", Part 3, American Journal of Health Promotion, 1989; 3: 5.

Pan American Union, "The Americas through their postage stamps", Bulletin of the Pan American Union, 1944; June: 356.

Pearn, P., "Paediatrica philatelica", Journal of Paediatrics and Child Health, 1999; 35:

232-236.

Pearn, J., "Further observation on the medical philately of Australia", Medical Journal of Australia, 1978; 2: 257-264.

Posnansky, M., "Propaganda for millions, images from Africa", African Arts, 2004; 37: 53-57.

Praestholm, J., "The origin and development of diagnostic radiology as illustrated by postage stamps", Acta Radiologica, 1997; 38: 930-936.

Pramanik, T., Pramanik, S., Chanda, R., "Postage stamps as a health promotion tool in the Nepalese community", Eastern Mediterranean Health Journal, 2004; 10: 442-444.

Raento, R., "Communicating geopolitics through postage stamps: the case of Finland", Geopolitics, 2006; 11: 601-629.

Raento, P., Brunn, SD., “Picturing a nation: Finland and postage stamps, 1917-2000", National Identities; 10: 49-75.

Raento, P., Stanley D. Brunn, SD., "Visualising Finland: postage stamps as political messengers", Geografika Annaler, Series B, Human Geography, 2005; 87: 145-163.

Reed, B., "Philately and the teaching of modern history", History, 1923; 7: 266-273.

Reid, DM., "The symbolism of postage stamps: a source for the historian", The Journal of Contemporary History, 1984; 19: 223-249.

Rose, G., Visual methodologies: an introduction to the interpretation of visual materials, London: Sage, 2007.

E. Sanitize, E., Shengelia, R., "First partnerships to fight tuberculosis", Clinical Research and Bioethics, 2015; 6: 5- 10.

Sanyal, U., "Stamping out cancer", British Journal of Cancer, 2003; 89: 2165-2171.

Senanayake, M., "Paediatric Philately", Archives of Disease in Childhood, 1997; 76: 287-288.

Senanayake, M., "Health messages on postage stamps", Ceylon Medical Journal, 2003; 48:

56.

Shampo, M., Edward C. Rosenow, EC., "A history of tuberculosis on stamps: Chest: 2009; 136: 578-582.

Sine, RL., "Stamps the alphabetical issues from ' $A$ ' to ' $D$ '”., The New York Times, 3 February 1985.

Smith, R., "Limits to medicine, medical nemesis: the expropriation of health". Journal of Epidemiology and Community Health. 2993; 57: 928.

Stocker, M., "The 'Smiling Boy' health stamps of 1931", Tuhinga, 2013; 24: 139-158.

Strauss, H., Politics, psychology and the postage stamp, Pennsylvania: American Philatelic Society, 1975;178-179.

Swan, G., Meade, T., Klein, JD., David, D., "Licking disability: reflections on the politics of postage stamps", Radical History Review, 2006; 6: 228-232. 
Tengland, P-A., "Behaviour change or empowerment: on the ethics of health-promotion", Public Health Ethics, 2012; 5: 140-153.

Vallgarda, S., "Nudge - A new and better way to improve health?" Health Policy, 2012;104: 200-203.

Wallack, L., "Media advocacy: a strategy for empowering people and communities", Journal of Public Health Policy, 1994; 15: 420-436.

Wallack, L., Dorfman, L., "Media advocacy: a strategy for advancing policy and promoting health", Health Education Quarterly, 1996; 23: 293-317.

White, J., "Chaco foes remain at odds", New York Times, 26 September 1937.

Woloshin, S. Schwartz, L., "The U.S. postal service and cancer screening - stamps of approval?", The New England Journal of Medicine, 1999;340: 884-887.

World Health Organisation, "Ottawa Charter for health promotion", Canada Journal of Public Health, 1986; 77: 425-430.

Wright, P., "Ivan Illich", The Lancet, 2003; 361: 185.

Wylie, A., Leedham-Green, K., 2017. "Health promotion in medical education: lessons from a major undergraduate curriculum implementation". Education for Primary Care, 2017; 28:

325-333.

Zeigler, D., "Post-communist eastern Europe and the cartography of independence", Political Geography, 2002;21: 671-686.

\section{Newspapers}

Meyer, EL., "Stamped out", The New York Times, 29 September 2017.

Mowbray, N., "Post Modern: why millennials have fallen in love with stamp collecting", The Observer, April 2020.

Lehmann, R., "The future of philately", Forbes Magazine, 28 August 2016.

Nelson, A., "Why stamp collecting is suddenly back in vogue", The Wall Street Journal, 5 June 2020.

Sine, RL., "Stamps the alphabetical issues from ' $A$ ' to ' $D$ '”., The New York Times, 3 February 1985.

\section{Unpublished Theses}

Dawson, A., Learning and curriculum design in community health nurse education, PhD Thesis, University of New South Wales, 2008.

Dawson, KP., Disputes over small territories, MA Thesis in Geography, Massey University, 2016.

Dawson, KP., Commerce or Commemoration? The role of the postage stamp in Great War remembrance, MA Thesis in History, Massey University, 2019.

Yardley, C.B. The representation of science and scientists on postage stamps, PhD Thesis, Australian National University, 2013.

\section{Uniform Resource Locator: Websites}


Arrojo source: https://www.stampboards.com/viewtopic.php?f=17\&t=51327\&start=150 Breast cancer stamp: https://www.illustrationhistory.org/illustrations/breast-cancer-stamp Breast feeding in Africa, http://www.flickr.com

Breast feeding in Africa (2) http://www.beauty-of-africa.tumblr.com

Breast feeding rates: https://www.who.int/health-topics/breastfeeding\#tab=tab 1

Canal Zone malaria:

https://postalmuseum.si.edu/exhibition/canal-zone-stamps-airmail-issues/7-cent-malariaeradication

Can Graphic Design Save Your Life?

https://www.itsnicethat.com/news/can-graphic-design-save-your-life-wellcome-trustexhibition-lucienne-roberts-rebecca-wright

Cigarette image: https://www.health.govt.nz/our-work/preventative-health-

wellness/tobacco-control/tobacco-standardised-packaging/tobacco-packaging

Development goals: https://sdgs.un.org/goals

Digital library, https://www.jstor.org/ accessed from 9/5/2020

Director-general of WHO statement:

https://apps.who.int/iris/bitstream/handle/10665/88730/EB34R18 eng.pdf?sequence=1\&is Allowed $=y$

Elias, P., How many postage stamps are there in the world? http://www.quora.com/PeterElias-7

Greely H., https://www.quotetab.com/quotes/by-horace-greeley

Herbert A. Friedman, HA., https://www.psywar.org/stamps.php

Health Promotion Agency (NZ).

https://www.healthed.govt.nz/search?topic\%5B0\%5D=48\&type=resource\&mode=pictureview

Health targets in New Zealand:

https://www.health.govt.nz/new-zealand-health-system/health-targets/about-health-

targets/health-targets-increased-immunisation

Indian proposal:

https://apps.who.int/iris/bitstream/handle/10665/100014/WHA3 91 eng.pdf?sequence=1

\&isAllowed $=y$

Insect-treated bed nets, Centres for Disease Control, USA.

https://www.cdc.gov/malaria/malaria worldwide/reduction/itn.html

JStor: https://www.jstor.org/

Limor, Y., Tamir, I., https://doi.org/10.1093/ct/qtz043

Magazine: https://www.indiatimes.com/news/india/all-those-complaining-about-

grihalakshmi-cover-must-see-this-indian-postal-stamp-from-1984

Malnutrition: https://www.who.int/nutrition/topics/world-food-day-2019-malnutritionworld-health-crisis/en/

Meghdoot cards: https://www.indianstampghar.com/category/philatelic-stationery/postalstationery/meghdoot-post-cards/

Ministry of Health, New Zealand: https://www.health.govt.nz/your-health/pregnancy-andkids/first-year/helpful-advice-during-first-year/breastfeeding-perfect-you-and-your-baby New Zealand skin cancer rates, https://www.sciencelearn.org.nz/resources/1329-newzealand-skin-cancer- statistics

Photograph of oral polio drops: 
http://polioeradication.org/wp-content/uploads/2016/07/13 Polio-imm-2-2.jpg

Polio eradication results: https://www.immune.org.nz/sites/default/files/polio-map 1988-

2014 616x312px.jpg

Polio in Pakistan: https://www.aljazeera.com/indepth/features/pakistan-polio-comebackvaccine-boycotts-191226142838246.html

Smallpox campaign: https://news.un.org/en/story/2020/05/1063582

Source of graph data: www.trussel.com/stamps/smoking/antismok.htm

Stamp releases:

https://www.post.gov.tw/post/internet/W stamphouse/index en.jsp?ID=2807\&file name= B077

Stamp use: https://csts.ua.edu/stamps/

The Smithsonian Institute's Postal Museum permits access for research into postal matters, https://postalmuseum.si.edu/

River blindness:

http://www.everydayhealth.com/infectious-diseases/onchoceriasis-river-blingness/

The University of Alabama - Stamp out smoking https://csts.ua.edu/stamps/airbrushed-

stamps/

United Nations small pox stamp: https://www.un.int/news/un-stamp-commemorates-40thyear-smallpox-eradication

Universal Postal Union, https://www.wnsstamps.post/en

United States stamps: https://facts.usps.com/stamps

World Health Organisation, Bangkok Charter for health promotion,

https://www.who.int/healthpromotion/conferences/6gchp/bangkok_charter/en/

https://www.who.int/healthpromotion/conferences/en/

World Health Organisation polio campaign:

https://www.who.int/countries/eth/areas/immunization/pei/en/ 